\title{
APPROACHES FOR UNVEILING THE KINETIC MECHANISMS OF VOLTAGE GATED ION CHANNELS IN NEURONS
}

A Dissertation
Presented to the
Faculty of the Graduate School
At the University of Missouri-Columbia

In Partial Fulfillment for the Requirements for the Degree of Doctor of Philosophy

\section{Submitted by MARCO ANTONIO NAVARRO}

Dr. Lorin Milescu, Dissertation mentor

May 2019 
(C) Copyright by Marco Navarro 2019

All Rights Reserved 
The undersigned, appointed by the dean of the Graduate School, have examined the

dissertation entitled

\section{APPROACHES FOR UNVEILING THE KINETIC MECHANISMS OF VOLTAGE GATED ION CHANNELS IN NEURONS}

presented by Marco Navarro,

a candidate for the degree of Doctor of Philosophy,

and hereby certify that, in their opinion, it is worthy of acceptance.

Professor Lorin Milescu

Professor Mirela Milescu

Professor David Schulz

Professor Kevin Gillis 


\section{Thesis Committee}

Dr. Lorin S Milescu

Assistant Professor, Department of Biological Sciences

University of Missouri, Columbia, MO

Dr. Mirela Milescu

Assistant Professor, Department of Biological Sciences

University of Missouri, Columbia, MO

Dr. David Schulz

Professor, Department of Biological Sciences

University of Missouri, Columbia, MO

Dr. Kevin Gillis

Professor, Department of Biological Engineering

Professor Medical Pharmacology and Physiology

University of Missouri, Columbia, MO 


\section{ACKNOWLEDGEMENTS}

For the love of science, may we stand tall on the shoulders of giants.

Thank you to all the laboratory animals who have been sacrificed for the sake of this work. Our progress towards understanding of physiology could not be achieved without this sacrifice.

I would like to thank those who supported me and kept me alive these many years, all the doctors, nurses, coaches, family, and friends.

Most of my inspiration in life stems from my parents. All the rocket launch videos and building model rockets drove my love for science and understanding and was fostered by my father's love for space and aeronautics. My mother was the best teacher I could ever ask for. She taught me to be a disciplined, well-rounded person that takes life for what it is and make the most of it. Thank you for always being there for love and support. Throughout all my years, my sister Jo and my brother Aaron have always challenged me to be the best person I could be. My family has always helped keep me in check. I'm not sure how one can get through the PhD process without a strong support system like them. They always know how to make me laugh and help find the small light of positivity in the vast darkness of space. I would never have been here without their encouragement and support to bring me to the best of my ability.

I would like to especially thank my mentors Lorin and Mirela Milescu. They shared with me their enthusiasm to understand ion channel biophysics. They stood by my side, always challenging me to think differently, explore new areas of science and always exposing me to new science and ideas. Your support through the years has kept me going steady, even when things get tough. They have taught me how to think things through and overall have made me a better person. I swear that I do not always, intentionally try to ignore you, all the time. Well... maybe I did a few times, but not on purpose, I promise.

I would like to thank the other strong educators in my life, who taught me to challenge the norms and to learn to solve problems. Each classroom along the way taught me to learn and think 
critically, rather than simple memorization. I would like to particularly mention Patrice Whalen, Jane Johnston, and Cheryl Lenox. As my first science teachers, they filled the classroom with fun and excitement, always pushing their students to ask critical questions in order to build an understanding of life. I also have to thank Dr. Daniel Warren for supporting the start of my scientific career. Through his mentorship, he shared his excitement with physiology and challenged me to pursue a $\mathrm{PhD}$.

I also would like to thank Dr. David Schulz and Dr. Kevin Gillis for serving as strong mentors, who challenged me to perform at the best of my ability. I would also like to thank Dr. Andy McClellan, Dr. Troy Zars, Dr. Michael Garcia, Dr. Jeffrey C. Smith, Dr. Richard Defazio and Dr. Analisa Scememi for their help in and guidance whenever I needed it.

I would never have been as productive as I have been without the generous support from my funding sources. I would like to particularly thank Dr. Mark Hannick, Dr. John David, and Debbie Allen as they made the transition to graduate school easy and their support throughout the years is priceless. I always thank them for the chance to travel and help give back to the community to recruit new students to Mizzou. I cherish many moments that would not be possible by the strong support and development of graduate students.

This dissertation would also never have happened without the strong presence of science throughout American culture. I would especially like to thank the creators/producers of Cosmos, the Magic School Bus, Pinky and the Brain, Bill Nye the Science Guy, Out of the Box, and Zoboomafoo. These TV shows were the backbone of my childhood entertainment and they inspired me to achieve scientific discovery, embrace new technology and most importantly learn to experiment. I would also like to thank the creators and producers of Jeopardy, Trivial Pursuit, and the GameShowNetwork, as these entertainment trivia shows keep our minds fresh. Without many of these platforms, I would not have had the drive and passion for science as I do now. I would like to thank my colleagues and support team at Mizzou especially Autoosa Salari, PhD, who as a wonderful mentor that set the bar high each week to challenge my ability and make me a 
strong scientist. I would also like to thank the Milescu Lab team of undergraduates that helped drive the spirit of science on our projects, mainly Benton Berigan, Jaime Hibbard, Jenna Lin, Michael Miller and Tyler Nivin. I would also like to mention the other undergraduate students who have always been great lab members: Tyler Carron, Ben Vega M.D., Brooklyn White, Paige Martinez, Kaydee Harper, Ben Zars, Megan Saracino, Taylor 'Gray’ Hallman, Connor Engel, Luke Cowan, and Hamza Iqbal.

The following lists members of my Mizzou family that must be named at this time. Thank you all the fun moments we have experienced and I hope to make many more in the future: Jake Burkhart, Vince Baggett, Benton Berigan, Marzie Armishneva, Tim Pale, Ronnie LeCombe, Laura Arnold, Elle Cook, Cindy Kyi, Kawasi Lett, Brian Lane, Nathan Harness, Megan Murphy, Gideon Ney, Eric Villalon, Tom Anderson, Alice Tipton, Mitch Tucker, Maria Jones, Aditi Mishra, Carlos Rivera, Daniel Kick, Joe Santin, Michael Gray, Austin Lynn, and Hedieh Attai. For the countless times we took a moment to relax and enjoy what life brings to us, Cheers!

I would also like to thank my other family of friends that were always willing to answer the phone and offer any guidance. Thank you for putting up with me and continuing to offer the valuable third-party advice that I often went to you for help. I would like to particularly mention all the members of the kik thread, Erin Carrol, Katie Montez, Maciej Gonek, and Ryan Paynok. I would also like to thank the support staff that was always there to help: Debbie Allen, Nila Emerich, Alan Marshall, Josh Hartley, Tyeece Little, Sadiya Aswad, and Waleed Atoud. 


\section{TABLE OF CONTENTS}

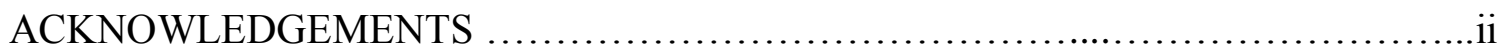

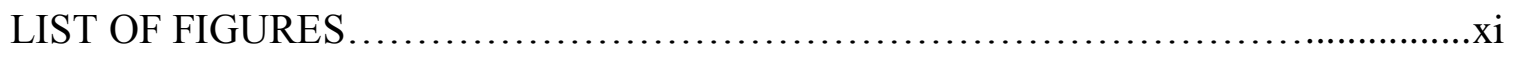

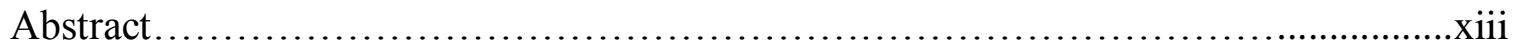

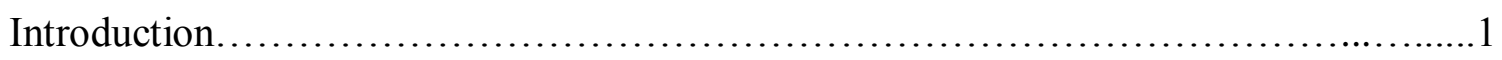

Chapter 1 Modeling the kinetic mechanisms of voltage-gated ion channels...........3

1.1. Introduction......................................................................................

1.2. Ion channel models................................................6

1.2.1 Kinetic mechanisms.......................................

1.2.2 Markov formalism.....................................9

1.2.3 Hodgkin-Huxley-type models.............................11

1.3. Solving ion channel models.........................................12

1.4. Experimental aspects............................................ 17

1.4.1 Voltage clamp protocol design............................17

1.4.2 Limitations of the recording system.........................19

1.4.3 Experimental artifacts.................................21

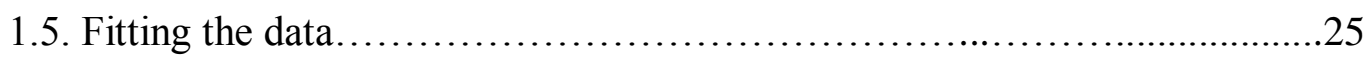

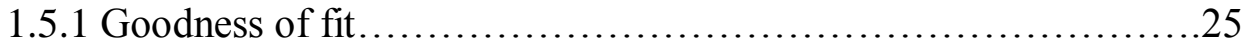

1.5.2 Computing the cost function.............................27

1.5.3 Model parameters......................................29

1.6. Dynamic clamp - testing models in live neurons........................30

1.6.1 Solving the model in real-time...............................31

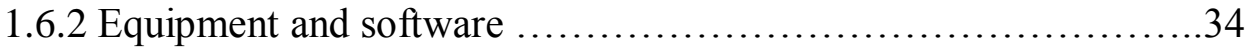


1.7. Conclusions

1.8. Figures and figure legends.

\section{Chapter 2. Estimating kinetic mechanisms with prior knowledge: linear and}

nonlinear parameter constraints....................................... 47

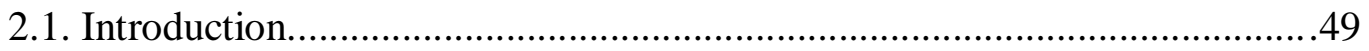

2.2. Materials and methods............................................. 52

2.2.1 Model parameters......................................52

2.2.2 Stochastic simulations...................................53

2.2.3 Model optimization...................................53

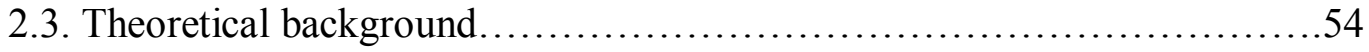

2.3.1 Kinetic mechanisms.......................................54

2.3.2 Formulating the topology of the model.......................56

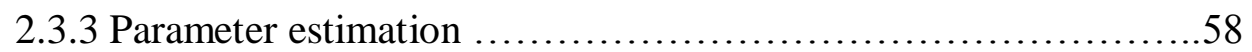

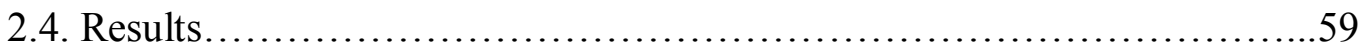

2.5. Implementing prior knowledge with linear parameter constraints..........60

2.5.1 Model assumptions.......................................61

2.5.2 Voltage-dependent and ligand-dependent rate constants..........64

2.5.3 Allosteric and other multiplicative factors....................67

2.5.4 Inequality constraints.................................69

2.5.5 Model parameters.......................................71

2.5.6 A general equation for linear parameter constraints..............72

2.5.7 Converting between model parameters and free parameters........73 
2.5.8 Redundant constraints.

2.5.9 Calculating the cost function and its gradients.

2.5.10 Calculating the error of the estimates....

2.6. Behavioral constraints and arbitrary parameter relationships... .86

2.6.1 Expanding the cost function.

2.6.2 Formulating behavioral constraints and arbitrary parameter

relationships.

2.6.3 Non-parametric behavioral constraints

2.6.4 A computational framework for solving behavioral constraints and

arbitrary parameter relationships..............................92

2.6.5 Testing the algorithm.....................................96

2.6.6 Applying linear parameter constraints......................97

2.6.7 Linear algebra calculations.................................101

2.6.8 Applying arbitrary constraints and behavioral constraint..........105

2.6.9 Optimizing the model..................................107

2.7. Discussion .................................................... 112

2.7.1 Compatibility with existing optimization frameworks...........114

2.7.2 Constrained fitting versus multi-objective fitting...............114

2.7.3 Model behavior: to enforce or not?.......................................115

2.8. Figures and figure legends....................................................... 118

Chapter 3. 3D data mapping and real-time experiment control and visualization in

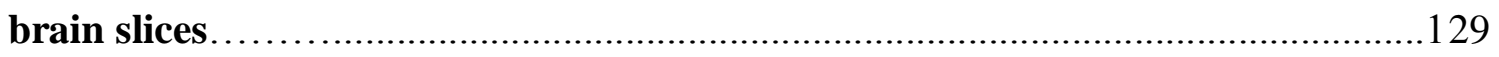

3.1. Introduction.................................................... 130 
3.2. Methodology.

3.2.1 Animal procedures and brain slice electrophysiology

3.2.2 Imaging hardware

3.2.3 Computer software and hardware

132

3.2.4 Software platform

3.2.5 $3 \mathrm{D}$ visualization.

3.3. Real-time 3D visualization and control of experiments.

3.3.1 Live imaging. 135

3.3.2 Motorized positioning. 136

3.3.3 Calibrating the motorized positioners. 138

3.4. 3D structural and functional data mapping... 141

3.4.1 Mapping the sample.

3.4.2 Data collection and visualization 142

3.5. Discussion 144

3.6. Figures and figure legends. 146

\section{Chapter 4. Investigating the role of intracellular calcium on voltage-gated sodium}

currents. 152

4.1. Background on voltage gated $\mathrm{Na}^{+}$channels 153

4.1.1 Nav channel function, selectivity, and pharmacology. 154

4.1.2 Nav channel subtype specification and expression. 155

4.1.3 Modulators of Nav channel kinetics.... 157

4.2. Chapter introduction 158

4.3. Materials and Methods 163 
4.3.1 Animal procedures........................................... 163

4.3.2 Brain slice preparation....................................... 163

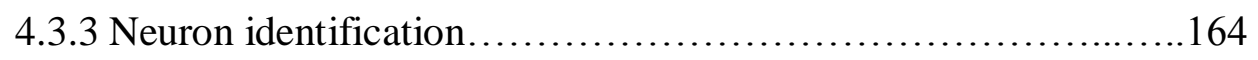

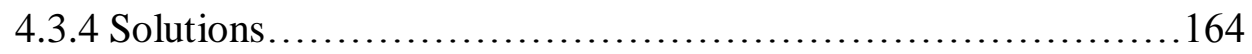

4.3.5 Pharmacology .............................................. 165

4.3.6 Electrophysiology ........................................... 166

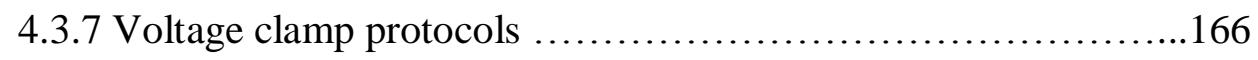

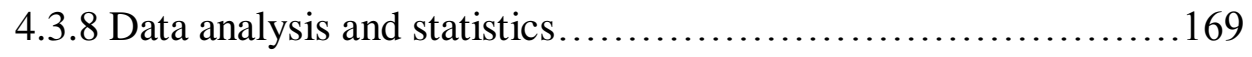

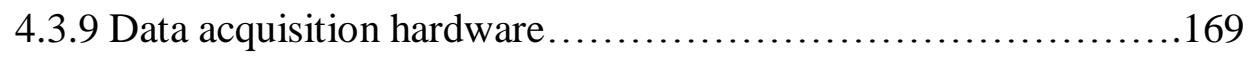

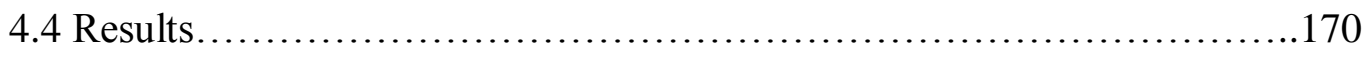

4.4.1 Steady-state activation and inactivation........................171

4.4.2 Recovery from inactivation...................................172

4.4.3 Use-dependent inactivation...................................173

4.4.4 Recovery from use-dependent inactivation.......................174

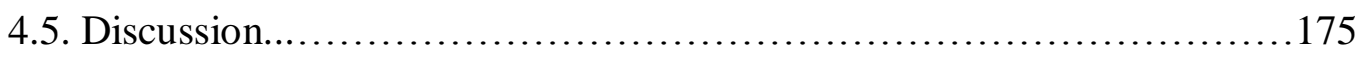

4.6. Figures and figure legends........................................ 177

\section{Chapter 5. Sodium channels implement a molecular leaky integrator that detects} action potentials and regulates neuronal firing ............................... 186

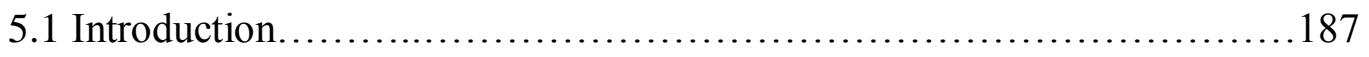

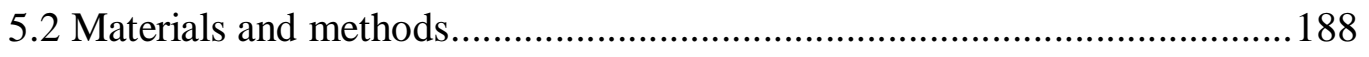

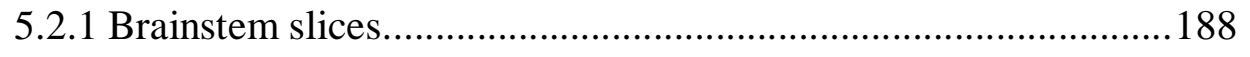

5.2.2 Acutely dissociated neurons.........................................................189

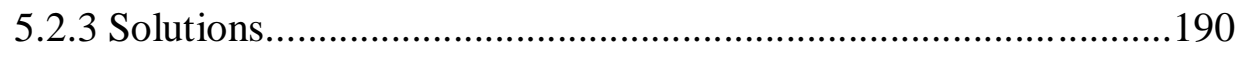




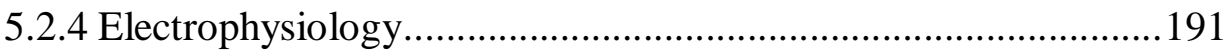

5.2.5 Voltage clamp experiments .........................................................192

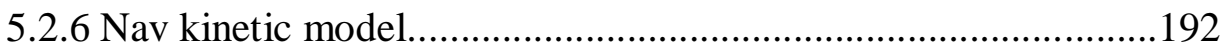

5.2.7 Computer simulations...................................................................193

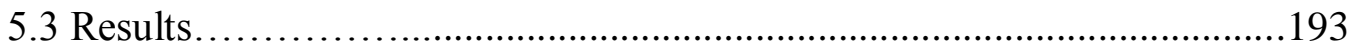

5.3.1 Action potentials in serotonergic raphe neurons and the

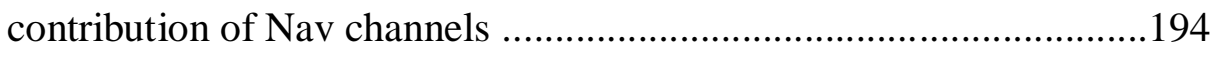

5.3.2 Nav channels have long-term inactivation...................................195

5.3.3 Mechanistic consequences of long-term inactivation...................196

5.3.4 Nav channels are a molecular leaky integrator..........................199

5.3.5 Nav channels detect action potentials and measure spiking

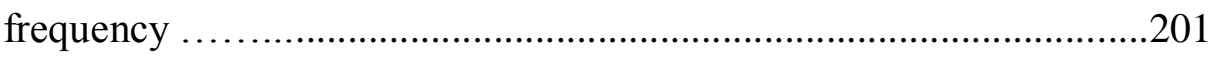

5.3.6 Nav channels drive spiking frequency..............................204

5.3.7 Nav channels are a molecular controller that regulates

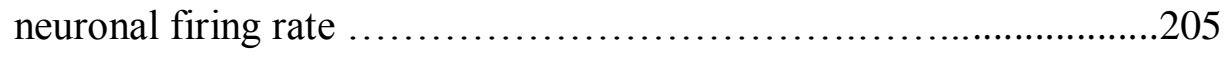

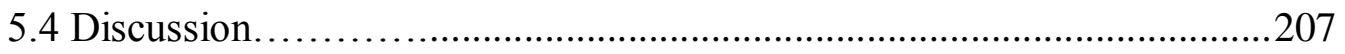

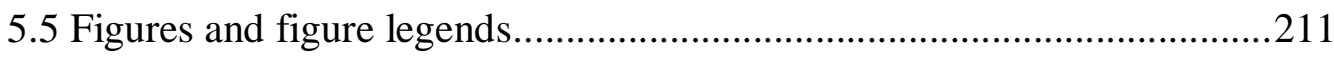

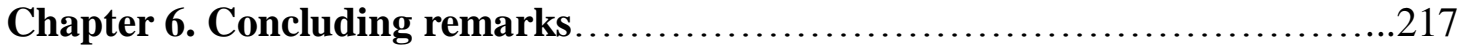

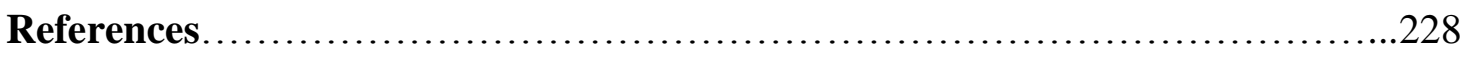

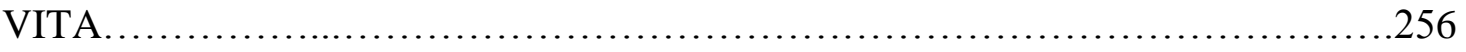




\section{LIST OF FIGURES}

Table

Page

Table 2.1. Model parameters and properties .125

Figure

Page

Figure 1.1. From model to data....................................................................4

Figure 1.2. Designing voltage-clamp protocols for Nav channels..........................42

Figure 1.3. Fitting the data with a model......................................................43

Figure 1.4. Testing ion channel models in live neurons with dynamic clamp.........44

Figure 1.5. Running dynamic clamp in Qub......................................................45

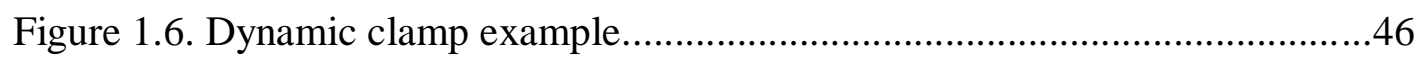

Figure 2.1. Estimating kinetic mechanisms with prior knowledge.......................118

Figure 2.2. Expressing prior knowledge via model topology and parameter relationships

Figure 2.3. Transformations between model parameters and free parameters.......120

Figure 2.4. Optimizing a model in QuB.....................................................121

Figure 2.5. Optimizing a constrained model................................................ 122

Figure 2.6. Test model with different sets of constraints................................123

Figure 2.7. Test data and model predictions.............................................. 124

Figure 2.8. Testing optimization with model constraints................................126

Figure 2.9. Enforcing model properties and behavior.....................................128

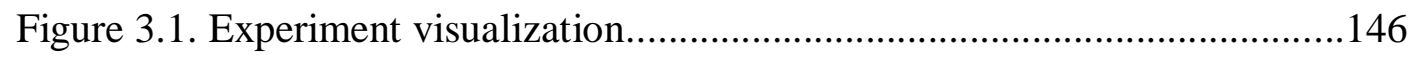

Figure 3.2. Live imaging with real-time position feedback...............................147

Figure 3.3. Online processing of functional imaging................................... 148 
Figure 3.4. Motorized pipette positioning.

Figure 3.5. Calibrating the motorized positioners 150

Figure 3.6. 3D data mapping. 151

Figure 4.1. Schematic of voltage-gated Nav channel structure 177

Figure 4.2. The respiratory brainstem preparation. 178

Figure 4.3. Isolation of $\mathrm{Na}^{+}$currents. 179

Figure 4.4. Testing activation and inactivation properties. 180

Figure 4.5. Steady State activation and inactivation kinetics. 181

Figure 4.6. Recovery from inactivation of $\mathrm{Na}^{+}$currents .182

Figure 4.7. Use-dependent inactivation with 100 pulses. 183

Figure 4.8. Use-dependent inactivation with voltage-clamp solutions. 184

Figure 4.9. Recovery after use-dependent stimulation.

Figure 5.1. Spontaneous firing in serotonergic raphé neurons and the contribution of Nav channels.

Figure 5.2. Nav channels in serotonergic raphé neurons exhibit a slow kinetic component

Figure 5.3. Nav long-term inactivation.

Figure 5.4. Nav channels implement a molecular leaky integrator that measures spiking frequency.

Figure 5.5. Nav channels drive spiking frequency in serotonergic raphé neurons.215 Figure 5.6. Nav channels can regulate spiking frequency in serotonergic raphé neurons in a negative feedback loop. .216 


\begin{abstract}
Ionic currents drive cellular function within all living cells to perform highly specific tasks. For excitable cells, such as muscle and neurons, voltage-gated ion channels have finely tuned kinetics that allow the transduction of Action potentials to other cells. Voltage-gated ion channels are molecular machines that open and close depending on electrical potential. Neuronal firing rates are largely determined by the overall availability of voltage-gated $\mathrm{Na}^{+}$and $\mathrm{K}^{+}$currents. This work describes new approaches for collecting and analyzing experimental data that can be used to streamline experiments.

Ion channels are composed of multimeric complexes regulated by intracellular factors producing complex kinetics. The stochastic behavior of thousands of individual ion channels coordinates to produce cellular activity. To describe their activity and test hypotheses about the channel, experimenters often fit Markov models to a set of experimental data. Markov models are defined by a set of states, whose transitions described by rate constants.

To improve the modeling process, we have developed computational approaches to introduce kinetic constraints that reduces the parameter search space. This work describes the implementation and mathematical transformations required to describe linear and non-linear parameter constraints that govern rate constants. Not all ion channel behaviors can easily be described by rate constants. Therefore, we developed and implemented a penalty-based mechanism that can be used to guide the optimization engine to produce a model with a desired behavior, such as single-channel open probability and use dependent effects.

To streamline data collection for experiments in brain slice preparations, we developed a 3D virtual software environment that incorporates data from micro-positioning motors and scientific cameras in real-time. This environment provides positional feedback to the investigator and allows for the creation of data maps including both images and electrical recordings. We have also produced semi-automatic targeting procedures that simplifies the overall experimental experience.
\end{abstract}


Experimentally, this work also examines how the kinetic mechanism of voltage gated $\mathrm{Na}$ channels regulates the neuronal firing of brainstem respiratory neurons. These raphe neurons are intrinsic pacemakers that do not rely on synaptic connections to elicit activity. I explored how intracellular calcium regulates the kinetics of TTX-sensitive $\mathrm{Na}^{+}$currents using whole-cell patch clamp electrophysiology. Established with intracellular $\mathrm{Ca}^{2+}$ buffers, high $\left[\mathrm{Ca}^{2+}\right]$ levels greater than $\sim 7 \mu \mathrm{M}$ did not change the voltage dependence of steady-state activation and inactivation, but slightly slowed inactivation time course. However, the recovery from inactivation and use dependence inactivation is slowed by high intracellular $\left[\mathrm{Ca}^{2+}\right]$. Overall, these approaches described in this work have improved data acquisition and data analysis to create better ion channel models and enhance the electrophysiology experience. 


\section{Introduction}

Cellular communication relies on the transduction of electrical and chemical signals across membranes. These signals are relayed by the activity of ion channels. Ion channels are integral membrane proteins that allow the passage of charged molecules through the hydrophobic membrane. The coordinated activity of these channels work to drive cellular activity that regulates metabolic functions, such as breathing, vison, and auditory processing. Voltage-gated channels utilize ionic gradients established by ionic pumps to elicit events known as action potentials (AP). Excitable cells within the body, such as muscles, neurons, and glia utilize APs to trigger further activity.

An action potential event is simply the coordinated opening and closing of individual ion channels that can sense voltage. A large charge distribution and fast change in voltage over milliseconds (AP) allows excitable cells to trigger the release of neurotransmitters, and intracellular calcium from intracellular stores, further activating second messenger pathways that amplify the single AP event to many other cellular pathways that can regulate gene expression. As these events are fast (ms) on the timescale of life, the frequency of APs is a better indicator as to overall activity.

The activity and best resolution of single channel events took place with the development of the patch-clamp technique, which utilizes a tight giga-ohm seal between glass and cell. This seal enhances the signal to noise ratio, so that with stable recordings one can measure a stochastic transition between two conductance levels. Within the noise of the data, physiologists confirmed what was previously hypothesized that aggregated openings of individual proteins are responsible for the $\mathrm{Na}^{+}$and $\mathrm{K}^{+}$currents that elicit an AP 
The ability to reliably record data from voltage-gated ion channels and other ionic proteins has propelled the field of neuroscience like no other. "Seeing into" the cellular activity of neurons allows for investigation to understand the circuitry that underlies certain behaviors. My behavior of interest is breathing, which requires the coordinated activity of muscles in the airways and diaphragm creating a negative pressure system to drive air across the lungs. This behavior is modular, as organisms must be able to respond to the environment and increase metabolic output. This behavior is driven by rhythmic circuits in the brainstem that can be isolated in a slice preparation and maintains coordinated activity under synaptic blockade.

This work aims to demonstrate how one can decode the kinetics of voltage-gated $\mathrm{Na}^{+}$ channels within neuronal circuits to develop an understanding of the regulators of intrinsic pacemakers. In order to further progress the field, one must also develop tools to take advantage of new technologies and develop new tools for analysis. 


\title{
Chapter 1
}

\section{Modeling the kinetic mechanisms of voltage-gated ion channels}

This chapter has been adapted from:

Salari, A., M.A. Navarro, and L.S. Milescu. 2016. Chapter 13: Modeling the Kinetic Mechanisms of Voltage-Gated Ion Channels. In: A. Korngreen (ed) Patch-Clamp Analysis: Advanced Techniques, pp 267-304, Springer, NY, USA

\begin{abstract}
Voltage-gated ion channels play a key role in action potential generation and propagation, in neurons and other excitable cells. Different types of neurons display unique patterns of cellular excitability and assemble into brain circuits with distinct network properties. The firing properties of individual neurons and neuronal circuits, and ultimately the function of the entire nervous system, are largely determined by the kinetic properties of voltage-gated Na channels. In this chapter, I discuss how to investigate these properties (state transitions, rate constants, voltage sensitivity, etc.) by generating ion channel models and testing them in live neurons using the dynamic-clamp technique. We focus on deriving biophysically realistic models from macroscopic currents obtained in whole-cell voltage-clamp experiments, using the Markov formalism. Nevertheless, the topics covered in this chapter are also useful for investigators interested in building Hodgkin-Huxley-type models or in understanding what information can be extracted from other types of electrophysiological data. Lastly, we discuss the technical
\end{abstract}


requirements for performing dynamic-clamp experiments and provide an example of this powerful experimental-computational approach.

Author contribution: M.A.N. ran simulations, made figures, and wrote the manuscript.

\subsection{Introduction}

Ion channels are the molecular building blocks of cellular excitability, forming highly specific and efficient pores in the membrane. Gated by various types of stimuli (chemical ligands, electricity, mechanical force, temperature, or light), ion channels form a superfamily of transmembrane proteins (Hille, 2001; Frank and Catterall, 2004). Channel activity underlies a vast number of physiological and pathological events. Voltage-gated ion channels are important in muscles, neurons, and many other cell types to both detect and drive changes in membrane potential, causing downstream responses (Bezanilla, 2000; Frank et al., 2005). As a more unusual example, the $\mathrm{Ca}^{2+}$ selective CatSper channels help drive sperm motility (Ren et al., 2001a; Qi et al., 2007) and the initial depolarization of the egg upon sperm fertilization (Xia and Ren, 2009). To perform all of these functions, the channel molecule undergoes conformational transitions within a set of conducting and non-conducting states, governed by specific kinetic mechanisms (Sigworth, 1994; Armstrong and Hille, 1998; Yellen, 1998).

The relationship between membrane voltage and ionic current is simple and can be derived from basic principles (Hodgkin, 1952). Electrically, the cell membrane is equivalent to a capacitor (lipid bilayer), which stores charge and is connected in parallel with variable conductances (ion channels), connected in series with a battery (the 
electrochemical potential of permeant ions across the membrane). If no external input is applied, the current flowing through the conductance and the current charging the capacitor sum to zero. Thus, ignoring spatial effects, the change in the membrane potential vs. time is proportional to the net ionic current flowing through the membrane, as described by the differential equation:

$$
\text { (1) } \quad C \frac{d V}{d t}=-I
$$

where $C$ is membrane capacitance, $V$ is membrane potential, $C \times d V / d t$ is the capacitive current that charges the membrane, $I$ is the ionic current flowing through the membrane, and $t$ is time.

All the ion channels within the membrane contribute to the total $I$, which is the algebraic sum of all the single-channel currents. Thus, any individual ion channel that opens or closes will cause an immediate and finite change in the net current $I$, unless $V$ happens to be equal to the reversal potential for that channel. From this perspective, a closing of a channel is as significant as an opening. In turn, a change in the overall current modifies the rate at which the membrane potential changes over time. As $V$ evolves in time, the driving force for the permeating ion and the kinetic properties of the voltage-gated channel will also change. These changes will again modify $I$, closing the causal loop between membrane potential and ionic current.

Because they both sense and control membrane potential, voltage-gated ion channels play a key role in action potential generation and propagation, in neurons and other excitable cells (Bean, 2007). Neurons, in particular, spend considerable amounts of chemical energy to create and maintain the electrochemical gradients necessary for action 
potentials to work (Richie, 1973; Hasenstaub et al., 2010) and establish communication within the nervous system. Such high energy demand requires support from astrocytes, which are required for proper maintenance of glutamate, lactate, ATP, D-serine and other neurotransmitters within the extracellular space (Pellerin and Magistretti, 1994;

Hülsmann et al., 2000; Bélanger et al., 2011; Tang et al., 2014). Different types of neurons display unique patterns of cellular excitability (Connors and Gutnick, 1990) and assemble into brain circuits with distinct network properties (Marder and Goaillard, 2006; Smith et al., 2007). The firing properties of individual neurons and neuronal circuits, and ultimately the function of the entire nervous system, are largely determined by the kinetic properties of voltage-gated ion channels (Getting, 1989; Harris-Warrick and Marder, 1991; Grillner, 2003; Prinz et al., 2004), which are further modified by pH, intracellular ligands, and auxiliary subunits (Woodhull, 1973; Li et al., 1992; Aman et al., 2009).

\subsection{Ion channel models}

Modeling ion channel kinetics can take the simple form of exponential curve fitting to find time constants, or sigmoid fitting of conductance curves. However, more sophisticated computational algorithms may be required when trying to extract information from a variety of experimental data. Whether this effort is worthwhile depends on the specific goals of the investigator. For example, one may want to find a model that can be used as a computational building block in large-scale simulations of neuronal networks. For this application, simplified phenomenological models will compute faster and would probably work just as well (Izhikevich and Edelman, 2008). However, with a more mechanistically-oriented goal, all biophysical knowledge available 
on a particular ion channel should be assembled into a detailed computational model (Armstrong, 2006), which can be tested and refined against new experimental data, and then further used to quantitatively test various hypotheses.

Starting with the seminal work of Hodgkin and Huxley (Hodgkin and Huxley, 1952; Catterall et al., 2012), most ion channel models fall somewhere in the range defined by these two examples. Although phenomenological models that simply describe the data are useful, the ultimate goal would be to quantitatively understand how the ion channel works at the molecular level and how it interacts with its environment at the cellular level. A biophysically realistic model must agree with existing theory and experimental data (Vandenberg and Bezanilla, 1991a; Hoshi et al., 1994; Zagotta et al., 1994a; Zagotta et al., 1994b; Schoppa and Sigworth, 1998a, b, c; Rothberg and Magleby, 2000), but it should also remain computationally tractable. Above all, we must keep in mind that "all models are wrong but some are useful" (Box, 1979).

\section{$\underline{1.2 .1 \text { Kinetic mechanisms }}$}

A kinetic mechanism is defined by a set of possible conformational states. Although in principle a protein can assume a continuum of structural conformations, statistically, the molecule will reside most of the time in a relatively small subset of high-occupancy states. The time spent continuously in a given state - the "lifetime" - is a random quantity with an exponential probability distribution (Colquhoun and Hawkes, 1995b). For voltage-gated ion channels, the high-occupancy states are the various conformations that correspond to functional and structural elements, such as resting or activated voltage sensors, closed or open pore, inactivated or non-inactivated channel, etc. (Bezanilla, 
2000; Catterall et al., 2007). A state can be identified experimentally if it is associated with a measurable change in properties (e.g., conductance, fluorescence), or it can be inferred statistically from the data, as some states may be characterized by subtle or less understood conformational changes.

A kinetic mechanism is further defined by a set of allowed transitions between states. Powered by thermal energy or other sources, the channel undergoes conformational changes at seemingly random times. Subsequent state transitions are also random events, with the average frequency of a given transition being inversely related to the energy barrier separating the two states (Colquhoun and Hawkes, 1982). These transition frequencies are quantified by rate constants. According to rate theory (Eyring, 1935), a voltage-dependent rate constant, $k_{\mathrm{ij}}$, corresponding to the transition from state $i$ to state $j$, has the following expression:

(2) $\quad \boldsymbol{k}_{\mathrm{ij}}=\boldsymbol{k}_{\mathrm{ij}}^{\mathbf{0}} \times \boldsymbol{e}^{\boldsymbol{k}_{\mathrm{ij}}^{\mathbf{1}} \times \boldsymbol{V}}$, where $V$ is the membrane potential and $\boldsymbol{k}_{\mathrm{ij}}^{\mathbf{0}}$ is the rate at zero membrane depolarization. $\boldsymbol{k}_{\mathrm{ij}}^{\mathbf{1}}$ is a factor that indicates how sensitive the rate constant is to voltage, as follows:

(3) $\quad \boldsymbol{k}_{\mathrm{ij}}^{\mathbf{1}}=\left(\boldsymbol{\delta}_{\mathrm{ij}}^{\mathbf{0}} \times \mathbf{z}_{\mathrm{ij}} \times \boldsymbol{F}\right) /(\boldsymbol{R} \times \boldsymbol{T})$, where $z_{\mathrm{ij}}$ is the electrical charge moving over the fraction $\delta_{\mathrm{ij}}$ of the electric field, $F$ is Faraday's constant, $R$ is the gas constant, and $T$ is the absolute temperature (Sigg, 2014). $\boldsymbol{k}_{\mathbf{i j}}^{\mathbf{1}}$ is zero for voltage-insensitive rates, while $\boldsymbol{k}_{\mathrm{ij}}^{\mathbf{0}}$ is zero for non-allowed transitions. Together, the set of possible states and the set of possible transitions describe the topology of a kinetic mechanism. The rate constants and their voltage dependence define the kinetic parameters of the mechanism. 


\subsubsection{Markov formalism}

Originally developed for stochastic processes (Baum, 1970; Gagniuc, 2017), the Markov formalism can be directly applied to ion channels, by mapping each known or hypothesized conformation of the channel into discrete states of the Markov model (Colquhoun and Hawkes, 1995b). The kinetic mechanism is mapped to a finite set of discrete conformations with exponentially distributed lifetimes and random conformational changes, which can be detected experimentally or inferred statistically. The rate constants associated with a Markov model can be compactly expressed as a rate matrix $\mathbf{Q}$, of dimension $N_{\mathrm{S}} \times N_{\mathrm{S}}$, where $N_{\mathrm{S}}$ is the total number of states. The $\mathbf{Q}$ matrix has each off-diagonal element, $q_{\mathrm{ij}}$, equal to the rate constant $k_{\mathrm{ij}}$. Each diagonal element, $q_{\mathrm{ii}}$ is equal to the negative sum of the off-diagonal elements of row $i$, so that the sum of each row of $\mathbf{Q}$ is zero. If a transition is not allowed between states $i$ and $j, q_{\mathrm{ij}}$ is zero.

The state of the model as a function of time can be conveniently expressed as a probability vector, $\mathbf{P}$. At any time $t$, each element of $\mathbf{P}$ represents the occupancy of that state, or the fraction of channels that reside in that state. Under stationary conditions, the average fraction of the total time spent by the channel in each state can be calculated as an equilibrium state occupancy. For an ensemble of channels, the average number of channels residing in state $i$ at equilibrium is equal to $p_{\mathrm{i}} \times N_{\mathrm{C}}$, where $p_{\mathrm{i}}$ is the equilibrium occupancy of state $i$ and $N_{\mathrm{C}}$ is the total channel count.

When conditions change (e.g., when a voltage step is applied in a voltage-clamp experiment), the energy landscape of the channel changes as well. All the voltagesensitive rate constants take different values, and thus the rate matrix $Q$ will change as 
well. As a result, the equilibrium state occupancies will also be different. For an ensemble of channels, if a state becomes less likely to be occupied under the new conditions, the fraction of channels residing in that state will decrease over time, at a rate that depends on the average lifetime of that state. The same behavior would be observed from repeated trials of a single channel. However, in a single trial, the channel will simply continue its stochastic behavior, just with different transition frequencies.

The process of relaxation towards a new state of equilibrium is described by the Kolmogorov equation (Cox and Miller, 1965), an ordinary differential equation (ODE):

$$
\text { (4) } \frac{d P}{d t}=P \times Q
$$

The state occupancies corresponding to equilibrium, $\mathbf{P}_{\mathrm{Eq}}$, can be obtained by setting the time derivative of $\mathbf{P}$ equal to zero and solving the resulting algebraic equation:

$$
\frac{d P_{\mathrm{eq}}}{d t}=P_{\mathrm{eq}} \times Q=0
$$

When conditions are stationary and the rate matrix $\mathrm{Q}$ is constant, the differential equation has a simple analytical solution:

$$
\text { (6) } P_{t}=P_{0} \times e^{Q \times t}
$$

where $\mathrm{P}_{\mathrm{t}}$ and $\mathrm{P}_{0}$ are the state occupancies at an arbitrary time $t$ and at time zero, respectively. The exponential of $\boldsymbol{Q} \times \boldsymbol{t}$ is another matrix, $\mathbf{A}$, that contains the conditional state transition probabilities. Each element of $\mathbf{A}, a_{\mathrm{ij}}$, is the conditional probability that the channel will be in state $j$ at time $t$, given that it was in state $i$ at time zero. No assumption is made about what other transitions would have occurred in that time interval. The 
transition probability matrix $\mathbf{A}$ for a given time $t$ can be calculated numerically using the spectral expansion method (Colquhoun and Hawkes, 1995b), as follows:

$$
A_{t}=e^{Q \times t}=\sum_{k} B_{k} \times e^{\lambda_{k} \times t}
$$

where the $\mathbf{B}_{\mathrm{k}}$ values are the spectral matrices derived from the eigenvectors of $\mathbf{Q}$, and the $\lambda_{\mathrm{k}}$ values are the eigenvalues of $\mathbf{Q}$, with $\lambda_{0}$ always equal to zero.

The $\mathbf{B}_{\mathrm{k}}$ and $\lambda_{\mathrm{k}}$ values can be calculated with a numerical library or with specialized software, such as Matlab or QuB. For analysis of macroscopic currents, it is convenient to calculate the transition probability matrix $\mathbf{A}_{\delta t}$ that corresponds to the data sampling interval, $\delta t$. Then, the state occupancies can be calculated recursively, starting with some initial solution, using a simple vector - matrix multiplication:

$$
\text { (8) } \quad \boldsymbol{P}_{t+\delta t}=\boldsymbol{P}_{t} \times \boldsymbol{A}_{\delta t}
$$

As discussed later in this chapter, when solving models in dynamic clamp applications, the A matrix can be precalculated across a physiological voltage range, to enable fast computation of the predicted current (Milescu et al., 2008).

\subsubsection{Hodgkin-Huxley-type models}

The ion channel models originally proposed by Hodgkin and Huxley (Hodgkin and Huxley, 1952) can also be formulated as Markov models, as they explicitly represent the closed, open, or inactivated states of the channel. While they were empirical at the time of their discovery, $\mathrm{HH}$ models remain to this day reasonably realistic and proficient. Their main limitation - but also their power, depending on the application - resides in making some strongly simplifying assumptions about the channel, which are simply 
outdated now (e.g., equal and independent "activation particles", or independent activation and inactivation processes). However, one should keep in mind that $\mathrm{HH}$ models are in disagreement with biophysical theory when their rate constants do not follow the Eyring rate theory (Eyring, 1935b), but instead are formulated as arbitrary functions of voltage, as discussed in (Strassberg and DeFelice, 1993; Milescu, 2005; Baranauskas and Martina, 2006). While their limited number of states and transitions would inherently reduce their ability to explain experimental data, $\mathrm{HH}$ models can gain more flexibility through these arbitrary rate functions.

\subsection{Solving ion channel models}

For mathematical illustration, let us consider the simple ion channel model shown in Fig. 1.1A. This model approximates a voltage-gated sodium (Nav) channel, featuring closed, open, and inactivated states. A single-channel stochastic simulation of a voltage-clamp recording is shown in Fig. 1.1B, where a noisy signal randomly jumps between zero and a tiny negative current (on the scale of pA). The noise in the trace is mostly caused by instrumentation, though the open state has its own intrinsic fluctuation in current (Sigworth, 1985), which can be estimated (Heinemann and Conti, 1992) and simulated (Qin, 2000a). On the basis of Ohm's law, the average single channel current corresponding to the open state can be calculated as follows:

(9) $\quad i=g \times\left(V-V_{R}\right)$,

where $g$ is the single channel conductance, $V$ is the membrane potential, and $V_{\mathrm{R}}$ is the reversal potential for the permeant ion. Note that Eq. 9 is an approximation: the current is 
a nonlinear function of voltage when the permeant ion has unequal intra- and extracellular concentrations, as described by the Goldman-Hodgkin-Katz current equation (Hille, 2001).

For channels that have several conducting states, we can make the unitary current equation more general by introducing a conductance vector $\mathbf{g}$, with each element $g_{\mathrm{i}}$ equal to the conductance of state $i$, or equal to zero for non-conducting states. The unitary current for an arbitrary set of state occupancies can be calculated by taking the dot product between the state occupancy vector $\mathbf{P}$ and the conductance vector $\mathbf{g}$, as a function of time:

$$
i_{t}=\left(\boldsymbol{P}_{t} \cdot \boldsymbol{g}\right) \times\left(V-V_{R}\right)
$$

When a single channel trace is simulated, at any given time only one element of $\mathbf{P}$ is equal to one, and the rest are zero. As the channel changes state during the simulation, a different element of $\mathbf{P}$ becomes equal to one, and thus a different conductance is "selected" by the dot product $\mathbf{P} \cdot \mathbf{g}$. To calculate the total ionic current, $I_{\mathrm{t}}$, given by an ensemble of identical channels, we simply multiply the unitary current by the total number of channels, $N_{\mathrm{C}}$ :

$$
I_{t}=\left(\boldsymbol{P}_{t} \cdot \boldsymbol{g}\right) \times\left(V-V_{R}\right) \times N_{C}
$$

Computationally, $I_{\mathrm{t}}$ can be efficiently calculated in two steps: first, calculate the state occupancies $\mathbf{P}_{\mathrm{t}}$, using the recursive Eq. 8; then, calculate $I_{\mathrm{t}}$ as a function of $\mathbf{P}_{\mathrm{t}}$, using Eq. 11. The time-invariant vector $\mathrm{g} \times\left(V-V_{\mathrm{R}}\right) \times N_{\mathrm{C}}$ needs to be recalculated only when the voltage changes. 
As shown earlier in Eq. 6, for a time interval where conditions are constant (e.g., during a voltage step), $\mathbf{P}_{\mathrm{t}}$ can be calculated as a function of some initial state occupancies, $\mathbf{P}_{0}$. For a typical voltage-clamp protocol, the $\mathbf{P}_{0}$ at the beginning of a sweep can be calculated as the equilibrium occupancies corresponding to the holding membrane potential. For this calculation to be accurate, the holding voltage should be maintained long enough to allow channels to reach equilibrium. If the protocol consists of a sequence of voltage step commands, the $\mathbf{P}_{0}$ of one step can be calculated as being equal to the $\mathbf{P}_{\mathrm{t}}$ at the end of the previous step. This idea could also be applied to protocols where the command voltage varies continuously (e.g., during a "ramp"). In this case, a continuously-varying episode can be approximated with a sequence of discrete steps of constant voltage. At the limit, each of these steps is as short as one acquisition sample.

Although very compact, Eq. 11 is not easy to interpret. To clarify its properties, we first replace $\mathbf{P}_{\mathrm{t}}$ with its solution as a function of $\mathbf{P}_{0}$ :

$$
I_{t}=\left(\left(\boldsymbol{P}_{0} \times e^{\boldsymbol{Q} \times t}\right) \cdot \boldsymbol{g}\right) \times\left(V-V_{R}\right) \times N_{C}
$$

Then, replace $e^{\mathrm{Q} \times t}$ with its spectral expansion:

$$
I_{t}=\left(\left(\boldsymbol{P}_{0} \times\left(\sum_{k=0}^{N_{S}-1} \boldsymbol{B}_{k} \times e^{\lambda_{k} \times t}\right)\right) \cdot \boldsymbol{g}\right) \times\left(V-V_{R}\right) \times N_{C}
$$

Rearrange the terms and obtain the total ionic current as follows:

$$
I_{t}=\sum_{k=0}^{N_{S}-1}\left(\left(\left(\boldsymbol{P}_{0} \times \boldsymbol{B}_{k}\right) \cdot \boldsymbol{g}\right) \times\left(V-V_{R}\right) \times N_{C} \times e^{\lambda_{k} \times t}\right) .
$$

Where $\mathbf{B}_{\mathrm{k}}$ is a spectral matrix derived from the eigenvectors of $\mathbf{Q}, N_{\mathrm{C}}$ is the number of channels, and $\lambda_{\mathrm{k}}$ is an eigenvalue of $\mathbf{Q}$. As all eigenvalues are negative, except for $\lambda_{0}$, all 
terms in Eq. 14 become vanishingly small when $t$ is sufficiently large, with the exception of the $\lambda_{0}$ term, which remains constant:

$$
I_{t \rightarrow \infty}=\left(\left(\boldsymbol{P}_{0} \times \boldsymbol{B}_{0}\right) \cdot \boldsymbol{g}\right) \times\left(V-V_{R}\right) \times N_{C}
$$

Since channels are at equilibrium when $t$ is sufficiently large, one can recognize that the vector $\left(\mathbf{P}_{0} \times \mathbf{B}_{0}\right)$ must be equal to $\mathbf{P}_{\mathrm{Eq}}$. Therefore, the current flowing at equilibrium has the expression:

$$
I_{\mathrm{Eq}}=\left(\boldsymbol{P}_{\mathrm{Eq}} \cdot \boldsymbol{g}\right) \times\left(V-V_{R}\right) \times N_{C}
$$

where: (17) $\boldsymbol{P}_{\mathrm{Eq}}=\boldsymbol{P}_{0} \cdot \boldsymbol{B}_{0}$.

In the equation above, $\mathbf{P}_{0}$ can be any arbitrary probability vector.

With these results, the macroscopic current can be written as:

$$
I_{t}=I_{\mathrm{Eq}}+\sum_{k=1}^{N_{S}-1}\left(I_{k} \times e^{\lambda_{k} \times t}\right)
$$

where $I_{\mathrm{k}}$ is a scalar quantity with dimension of current:

$$
I_{k}=\left(\left(\boldsymbol{P}_{0} \times \boldsymbol{B}_{k}\right) \cdot \boldsymbol{g}\right) \times\left(V-V_{R}\right) \times N_{C}
$$

The eigenvalues, $\lambda_{\mathrm{k}}$, can be replaced with time constants, $\tau_{\mathrm{k}}$, obtaining the final current equation:

$$
\text { (20) } \quad I_{t}=I_{\mathrm{Eq}}+\sum_{k=1}^{N_{S}-1}\left(I_{k} \times e^{-t / \tau_{k}}\right) \text {, }
$$

where $\tau_{\mathrm{k}}=-1 / \lambda_{\mathrm{k}}$. The macroscopic current described by Eq. 20 as a function of time is a sum of $N_{\mathrm{S}}-1$ exponentials, plus a constant term. Each exponential component is parameterized by a time constant $\tau_{\mathrm{k}}$ and an amplitude $I_{\mathrm{k}}$. 
These results are general: any voltage-gated ion channel that has $N_{\mathrm{S}}$ high-occupancy conformations will in principle generate a macroscopic current with $N_{\mathrm{S}}-1$ exponentials, when subjected to a step change in membrane potential. This is illustrated in Fig. 1.1C for the simple three-state model: $I_{\mathrm{t}}$ (the red trace) has the expected profile of rise (activation) followed by decay (inactivation). This time course is a sum of two simple exponential components that vanish to zero with different time constants. In this particular case, $I_{\mathrm{Eq}}$ is almost zero.

In the above equations, the macroscopic current $I_{\mathrm{t}}$ was calculated as a deterministic function of some initial conditions $\mathbf{P}_{0}$. However, one should keep in mind that $I_{\mathrm{t}}$ is the sum of many unitary currents, each generated by an individual ion channel that makes random transitions between states. These stochastic events at the single channel level will make the macroscopic current a stochastic process as well. Therefore, the state occupancy at time $t$ is a random quantity, characterized by a probability distribution (Celentano and Hawkes, 2004; Milescu et al., 2005; Moffatt, 2007; Stepanyuk et al., 2014). The state at time $t$ can be statistically predicted from some known previous state, but the uncertainty of the prediction increases with the time from the reference point. In contrast, the initial state of a deterministic process can predict any future state with equal precision. The difference between stochastic and deterministic processes is illustrated in Fig. 1.1C, where the trajectory of the stochastically simulated macroscopic current (black trace) consistently diverges from the deterministically calculated current (red trace). 


\subsection{Experimental aspects}

\subsubsection{Voltage clamp protocol design}

Single channel or macroscopic voltage-clamp recordings elicit stochastically fluctuating current, mixed with noise and artifacts. In single channel recordings, the mean value of the current randomly jumps between two (or more) levels, often by as little as $1 \mathrm{pA}$, corresponding to molecular transitions between conducting and non-conducting channel conformations. For example, in the single channel trace shown in Fig. 1.1B, there happens to be four conductance changes over $50 \mathrm{~ms}$. A channel with faster kinetics would result in more transitions per second, or, equivalently, in shorter average lifetimes in each state. Furthermore, a channel with greater voltage sensitivity would exhibit transition frequencies that change more substantially with voltage. Overall, the statistical properties of single-channel data can be analyzed with a variety of mathematical methods and computational algorithms, to extract the kinetic mechanism of the underlying ion channel (Ball and Sansom, 1989; Hawkes et al., 1990; Qin et al., 1996, 2000a; Venkataramanan and Sigworth, 2002; Qin and Li, 2004; Csanady, 2006)

Voltage-clamp protocols must be designed carefully and optimized to minimize these issues. Overall, the most important practical recommendation we can make is to design and apply as many types of stimuli as feasible, to force the channel to visit as many states as possible, which should result in well-observed exponential components and welldetermined kinetic parameters. Ultimately, designing a good set of stimulation protocols is an iterative process, without a priori solutions. It may well happen that applying yet 
another protocol exposes a new behavior of the channel, which then needs to be investigated with new or refined stimuli.

An example of a typical set of voltage-clamp protocols is given in Fig. 1.2, as applied to recording whole-cell Nav currents from mammalian neurons in brain slices (Milescu et al., 2010b). A minimum of four protocols is necessary to investigate the kinetic properties of Nav channels, as illustrated in panels A, B, C, and D. Each of these voltage-clamp protocols forces the channel on a different state trajectory, thus exposing a different set of kinetic properties. For example, the protocol in panel A starts the channel in a state of deactivation, and takes it through activation, opening, and inactivation. Several exponential components are well defined in the data, particularly the two time constants of inactivation. In contrast, the protocol in panel $\mathrm{C}$ starts the channel in a state of deactivation as well, but the channel is taken directly into inactivation, without opening. Two time constants of inactivation can also be detected in the data, but the exponential components have lower amplitude and thus are slightly less well defined.

With some of these protocols, the raw data can be used directly to determine the kinetic parameters (e.g., the time course of activation and inactivation in panel 1.2A). With others, the raw data are first processed to extract some empirical measure of state occupancy, which is then used to estimate kinetic parameters. Examples are the (pseudo) steady-state activation and inactivation in panel $\mathrm{E}$, and the time course of recovery from inactivation and the sub-threshold inactivation in panel F. Generally, the raw data are used directly when an exponential time course is experimentally observable in the macroscopic current. For example, when the channel activates, opens, and then 
inactivates (panel A). When state changes are not associated with changes in conductance, information is obtained from two-pulse protocols. For example, when the channel inactivates at membrane potentials where it cannot activate and open (panel C). In this case, the peak of the current is used as an empirical measure for the total occupancy of non-inactivated states, available to generate current upon activation.

\subsubsection{Limitations of the recording system}

Depending on the recording technique, the experimental preparation, and the noise levels of the recording system, a change in the conductance state of a single channel may be very difficult or impossible to detect experimentally. Second, the frequency of transitions in the overall state of the ensemble is proportional to the total number of channels, and it may exceed the bandwidth of the recording system. For example, if the average single channel transition frequency is $10 \mathrm{~s}^{-1}$, an ensemble of 10,000 channels would exhibit 100,000 transitions per second, while the recording bandwidth $(50 \mathrm{kHz}-100 \mathrm{kHz})$ may be limited to lower or similar magnitude. Thus, information encoded in the magnitude and frequency of stochastic current fluctuations may be lost.

A generally more reliable source of information is the mean value of the macroscopic current, as a function of time and voltage (Anderson and Stevens, 1973; Sigworth, 1980a). Although the mean value can be easily extracted from noisy data, even in this case, decoding the kinetic mechanism is far from being trivial. The main difficulty lies in the ambiguous relationship between the exponential parameters describing the macroscopic current (time constants $\tau_{\mathrm{k}}$ and amplitudes $I_{\mathrm{k}}$ ) and the kinetic parameters of the channel (rate constant factors $k_{\mathrm{ij}}^{0}$ and $k_{\mathrm{ij}}^{1}$ ). 
Following a step change in conditions, the overall state of the ensemble relaxes exponentially towards a new equilibrium. For a channel with $N_{\mathrm{S}}$ states, this relaxation process is quantified by a set of $2 \times N_{\mathrm{S}}-1$ parameters, as described by Eq. 20 : $N_{\mathrm{S}}-1$ time constants $\tau_{\mathrm{k}}, N_{\mathrm{S}}-1$ amplitudes $I_{\mathrm{k}}$, and the equilibrium current $I_{\mathrm{Eq}}$. Every one of these exponential parameters, including $I_{\mathrm{Eq}}$, is a mathematical function of all the rate constant parameters, and implicitly a function of membrane potential. Thus, while calculating the exponential parameters from the kinetic mechanism is straightforward, the inverse calculation is not. Furthermore, this also implies that no more than a maximum of $2 \times N_{\mathrm{S}}-$ 1 kinetic parameters can be extracted from a macroscopic current generated in response to a single voltage step. In reality, kinetic mechanisms may have more parameters than that. For example, the three-state model in Fig. 1.2A has eight kinetic parameters but only five exponential parameters. Even with this unrealistically simple model, it is clear that the kinetic parameters of the model cannot be unequivocally determined from the mean value of the macroscopic current, unless the voltage clamp protocol is expanded to more than one voltage step.

Another difficulty is related to the theoretical and experimental observability of all the exponential components, given the limited resolution of the recording system. Although each pair of exponential parameters $\left(\tau_{\mathrm{k}}, I_{\mathrm{k}}\right)$ depends on all the rate constants, fast or slow exponential components will be influenced most by similarly fast or slow rates, respectively. Then, if a certain exponential component is weakly represented in the data, some of the kinetic parameters will also be weakly determined. The contribution of an exponential component to the data, given a set of kinetic and conductance properties, 
depends on two factors. First, the amplitudes $I_{\mathrm{k}}$ depend on the initial state occupancies $\mathrm{P}_{0}$. Thus, depending on the voltage-clamp protocol, some components may have very small, or even zero, amplitude, and can be undetectable relative to the experimental resolution. Overall, a change in state occupancy is accompanied by a change in current only if the total occupancy of the conducting states changes. If this fraction doesn't change, or if the change is small relative to the resolution of the recording system, the mean current value will remain approximately constant, even though the properties of the stochastic fluctuations may change. Second, an exponential component can be observed experimentally only if the bandwidth of the recording system is adequate. Thus, very fast $(<500 \mu \mathrm{s})$ exponentials may be distorted or filtered out, while very slow components may not be detected in short protocols. A property worth remembering is that these exponential components vanish in order, from the smallest to the largest time constant. As a result, the fastest components will be affected the most by experimental artifacts associated with abrupt changes in the command voltage.

\subsubsection{Experimental artifacts}

The data recorded in voltage-clamp experiments do not contain just the current of interest, but are contaminated by a variety of artifacts, including other currents active in the preparation, experimental noise, voltage-clamp errors, etc. (Marty and Neher, 1995). All of these artifacts will negatively affect fitting algorithms and can result in a distorted model. Although artifacts cannot be eliminated, they can be reduced to acceptable levels. Thus, the effects of random measurement noise, which lowers the precision of parameter estimates, can always be reduced by collecting more data, and generally are not an issue. 
Deterministic power line interference (50 or $60 \mathrm{~Hz}$ and harmonics) can be easily removed online or offline. Uncompensated brief transients that occur when the command voltage changes abruptly can simply be excluded from the fit, provided that they don't overlap with significant channel activity. However, longer transients must be somehow separated from the signal. Voltage-clamp errors caused by incomplete compensation of the series resistance could be significant, as the actual voltage at the membrane is not truly measured with the patch-clamp technique (Sigworth, 1995). The membrane voltage is estimated from the measured series resistance and the recorded current. Then, a corrected version of the command voltage protocol can be constructed and used in the data fitting procedure.

As explained above, an arbitrary voltage waveform can be approximated with a sequence of constant voltage steps. Another artifact is imperfect space-clamp, which can occur when recording from neurons in vivo or in brain slices (Bar-Yehuda and Korngreen, 2008). In this case, the current recorded from the soma can be contaminated with action potentials back-propagating from the axon (Stuart et al., 1997a), which usually escapes voltage-clamp control. Space-clamp errors can be reduced with a simple technique that selectively inactivates axonal sodium channels and thus makes the axon a passive compartment (Milescu et al., 2010a). Finally, the bandwidth of the recording system should be sufficiently wide for the kinetics of the ion channel investigated. Initially, the cutoff frequency of the low-pass filter and the sampling rate of the digitizer should be set as high as possible to identify the fastest time constant present in the data. Then, acquisition parameters can be set to the values recommended by Nyquist's sampling 
theorem (Heinemann, 1995). In many cases, the fastest time constant corresponds to channel activation or deactivation.

Even when these artifacts cannot be eliminated, in principle they can be parameterized and included into the fitting algorithm. Unfortunately, contamination with other ionic currents that are active in the preparation cannot be easily encoded in the algorithm. These currents are generally unknown quantities and cannot be compensated computationally. The ideal solution is to isolate the current of interest pharmacologically, with a very specific blocker. In the case of Nav channels, TTX is very potent and selective for central nervous system Nav channel subtypes. To truly isolate a current of interest, the same protocols can be repeated under control conditions and with a saturating dose of TTX $(1 \mu \mathrm{M})$ applied (Nav channels have an $\mathrm{IC}_{50} \sim 200 \mathrm{nM}$ for TTX). Then, the current of interest can be obtained by performing a subtraction between the two data sets. This subtraction not only eliminates all other currents, including leak, but will also remove capacitive transients. However, not all channels can be completely isolated by pharmacological subtraction, as many agents are promiscuous, with off target effects at higher concentrations (Rosker et al., 2007).

As a general solution, all other currents should be reduced as much as possible. Some currents can be blocked pharmacologically, while others can be rendered inactive by exploiting voltage-dependence with clever protocol design. Furthermore, the background leak currents and possibly other currents left unblocked can be subtracted using the P/n technique (Armstrong and Bezanilla, 1974), which will also remove capacitive transients. 
However, one should be aware that the P/n method relies on the assumption that leak currents are linear with voltage, and thus cannot subtract voltage-sensitive currents.

When designing protocols to isolate the current of interest, pharmacologically or via the $\mathrm{P} / \mathrm{n}$ technique, one should keep in mind that these procedures will extend the total acquisition time, and recording parameters may change over time. For example, the seal resistance may deteriorate, causing an increase in leak, the level of solution in the bath may change and alter pipette capacitance and transients, and series resistance may fluctuate and change the amplitude of recorded currents. Generally, currents may run down over time. All these changes will distort the subtracted current. One other problem with subtraction methods is that uncompensated series resistance errors depend on the total current flowing. Then, if the total current takes significantly different values under control versus pharmacological block or $\mathrm{P} / \mathrm{n}$ conditions, the actual voltage at the membrane will also differ. As a result, the subtracted current will be contaminated with some leftover current. Thus, even if a good blocker is available for the channel of interest, reducing all the other currents pharmacologically is still recommended. A similar artifact occurs when the current of interest is functionally coupled with other currents (e.g., $\mathrm{Ca}^{2+}-$ activated $\mathrm{K}^{+}$currents). These would no longer be activated when the current of interest is blocked. Finally, one should keep in mind that when two random variables are subtracted from each other, their mean values subtract but their variances add. Thus, subtracting two sets of currents will result in a signal with greater noise, which would make it difficult to apply fitting methods that rely on the properties of current fluctuations. 


\subsection{Fitting the data}

The parameter estimation procedure combines a parameter optimization engine (the optimizer) and an algorithm that calculates how well the model explains the data (the cost function, or the goodness of fit). The optimizer starts with a set of initial values and iteratively explores the parameter space, according to a defined search strategy, until it finds a set of parameters that maximizes the goodness of fit. For each point sampled in the parameter space, the optimizer calls the estimation algorithm to evaluate the goodness of fit. Typically, the optimizer is data- and model-blind, although it can be tweaked for a particular problem. A variety of general optimization algorithms that have been described in the literature (Fletcher, 2013) and are available in numerical libraries can be applied to ion channels. In contrast, the function that calculates the goodness of fit is very specialized and can be quite complicated.

\section{$\underline{1.5 .1 \text { Goodness of fit }}$}

In general, how well the model explains the data can be defined in different ways, depending on the data and the model. For a deterministic time series contaminated by measurement noise, the goodness of fit is typically given by the sum of squared errors, $S$, between the experimental data points and the fit curve:

$$
S=\sum_{t}\left(y_{t}-f_{t}(\boldsymbol{M}, \boldsymbol{K})\right)^{2}
$$

where $y_{\mathrm{t}}$ is the data point measured at time $t$ and $f_{\mathrm{t}}(\mathbf{M}, \mathbf{K})$ is the calculated value at time $t$, given a structural model $\mathbf{M}$ and a set of parameters $\mathbf{K}$. The best fit parameters are those that correspond to the lowest $S$ value that could be reached. 
Curve fitting is not the ideal method for data generated by ion channels, or other stochastic processes, as these data are not simply described by deterministic functions and thus cannot be theoretically calculated at every time point. Instead, ion channel data are a stochastic sequence of channel states, contaminated with random measurement noise. Nevertheless, this stochastic sequence is generated by a probability distribution (Colquhoun and Hawkes, 1995b). For single-channel data, this probability distribution can be used to calculate the likelihood of the data, $L$ (Hawkes et al., 1990):

$$
\text { (22) } L=p(\boldsymbol{Y} \mid \boldsymbol{M}, \boldsymbol{K}) \text {, }
$$

where $p$ is the conditional probability of the data sequence $\mathbf{Y}$, given a model topology $\mathbf{M}$ and a set of parameters $\mathbf{K}$. The best fit parameters correspond to the highest $L$ value that could be reached. In practice, the logarithm of the likelihood function is used instead of the likelihood itself, because $L$ may reach intractably small or large values.

Ideally, macroscopic currents should also be approached as a stochastic process, using a likelihood-based goodness of fit. A variety of mathematical and computational algorithms have been designed to calculate the likelihood of a macroscopic current (Celentano and Hawkes, 2004; Milescu et al., 2005; Moffatt, 2007; Stepanyuk et al., 2014b), all making various approximations to speed up the computation. Ultimately, the fastest but theoretically the least accurate approximation that can be made is to completely ignore the stochastic nature of the macroscopic current. Essentially, the goodness of fit in this case is calculated as the sum of squared errors between the experimental data and the calculated macroscopic current, $I_{\mathrm{t}}$ :

$$
S=\sum_{t}\left(y_{t}-I_{t}\right)^{2} .
$$


This approximation is most suitable when the analyzed current is generated by many channels, when stochastic fluctuations are small relative to the mean value and comparable to the measurement noise. All other methods that make more accurate assumptions exploit in some way the fluctuations of the current, and theoretically should produce more accurate or more precise parameter estimates. However, as discussed above, many experimental data are not clean enough for noise analysis and the mean of the current may be the only reliable source of information. This condition describes well the macroscopic currents generated by voltage-gated ion channels in whole-cell patchclamp experiments (Fig. 1.3).

\subsubsection{Computing the cost function}

For each data set that is included in the fitting procedure, the estimation algorithm must calculate the goodness of fit. When the cost function is the sum of squared errors, $S$, then the mean current $I_{\mathrm{t}}$ must be calculated for every point in the data. Essentially, the algorithm must simulate a macroscopic current in response to the same voltage-clamp protocol as was used to record the data, given the set of parameters proposed by the optimizer in that iteration. For two-pulse protocols, such as those shown in Fig. 1.2, the simulated current sequence must also be processed in the same way as the experimental data. For example, the experimental recovery from inactivation (Fig. 1.2D) is calculated, as a function of time and recovery potential, as the ratio between the peak current obtained with the test pulse and the peak current obtained with the conditioning pulse. Although it might be tempting, it is a bad idea to calculate the theoretical recovery from inactivation using the sum of non-inactivated state occupancies. Instead, it should be 
calculated as for the experimental curve: first, simulate the response of the model to the two-pulse protocol, then, from this simulation, calculate the ratio of the two peaks.

$I_{\mathrm{t}}$ is most efficiently computed recursively, using Eq. 8 to calculate $\mathbf{P}_{\mathrm{t}+1}$ from $\mathbf{P}_{\mathrm{t}}$, where $t$ and $t+1$ refer to consecutive samples. The computation is initialized with a $\mathbf{P}_{0}$ calculated as the equilibrium state occupancy vector that corresponds to the holding voltage. The entire sequence of operations can be summarized as follows:

$$
\begin{aligned}
& \boldsymbol{P}_{\mathrm{Eq}}=1 \cdot \boldsymbol{B}_{0, V_{H}}, \\
& \boldsymbol{P}_{0}=\boldsymbol{P}_{\mathrm{Eq}}, \\
& \boldsymbol{P}_{1}=\boldsymbol{P}_{0} \times \boldsymbol{A}_{\delta \mathrm{t}, \mathrm{V}_{1}}, \\
& \ldots \\
& \boldsymbol{P}_{t}=\boldsymbol{P}_{\mathrm{t}-1} \times \boldsymbol{A}_{\delta \mathrm{t}, \mathrm{V}_{t}}, \\
& I_{t}=\boldsymbol{P}_{t} \times \boldsymbol{I}_{V_{t}}, \\
& S_{t}=\left(y_{t}-I_{t}\right)^{2},
\end{aligned}
$$

where 1 is the normalized unity vector, with each element equal to $1 / N_{S} ; \boldsymbol{B}_{0, V_{H}}$ is the spectral matrix corresponding to $\lambda_{0}$ and calculated for a voltage equal to the holding potential, $V_{\mathrm{H}} ; \boldsymbol{A}_{\delta \mathrm{t}, \mathrm{V}_{t}}$ is the transition probability matrix calculated for $\delta t$ and a voltage equal to the command potential at time $t, V_{\mathrm{t}} ; S_{\mathrm{t}}$ is the squared error at time $t$. Finally, $\boldsymbol{I}_{V_{t}}$ is a vector with dimension of current, with each element equal to the maximum current that would be generated if all the channels resided in that state:

$$
\boldsymbol{I}_{V_{t}}=\boldsymbol{g} \times\left(V_{t}-V_{R}\right) \times N_{C}
$$


When the command voltage changes during a protocol sequence, the spectral matrix $\mathbf{B}_{0}$ and the transition probability matrix $\mathbf{A}_{\delta t}$ are replaced with the matrices calculated for that voltage value. As discussed, instead of the command voltage, one could use the actual voltage measured at the membrane, when available, or a voltage corrected for errors caused by the uncompensated series resistance.

The total sum of squared errors, $S$, is the sum of squared errors for all data points used in the analysis. $S$ could be divided in components corresponding to individual data sets, each multiplied by a weighing factor:

$$
\text { (26) } S=\sum_{i} w_{i} \times S_{i} \text {. }
$$

These weighing factors can be chosen empirically, to establish the relative contribution of each data component to the cost function.

\subsubsection{Model parameters}

For a given model topology, the unknown parameters to be determined are the rate constant factors $k_{\mathrm{ij}}^{0}$ and $k_{\mathrm{ij}}^{1}$. However, the macroscopic current depends on additional quantities: the unitary conductance, g, and the total number of channels, $N_{\mathrm{C}}$. Calculating $I_{\mathrm{t}}$ in the cost function requires these quantities. Normally, for a given ion channel type, the unitary conductance has the same value in every recording and can be estimated directly from single channel data, or via noise analysis from macroscopic currents (Sigworth, 1980a; Heinemann and Conti, 1992). Although $N_{\mathrm{C}}$ can also be determined through noise analysis, it takes a different value in each experimental preparation and it cannot always be known. One possibility is to make $N_{\mathrm{C}}$ a parameter to be estimated 
(Milescu et al., 2005). If the data used in the fit were obtained from multiple experiments, then there will be multiple $N_{\mathrm{C}}$ parameters, one for each preparation. The downside with this approach is a potentially large increase in the dimensionality of the parameter space, which would slow down the optimizer. Another possibility is to normalize the current in each data set to the local maximum value. The disadvantage in this case is a greater ambiguity in the estimated kinetic parameters. Furthermore, it can be problematic to analyze fluctuations. With some models, distinct combinations of rate constants and channel count values can generate the same macroscopic current in response to a voltageclamp protocol (Milescu et al., 2005). However, in principle this ambiguity could be resolved by adding more protocols to the fit.

\subsection{Dynamic clamp - testing models in live neurons}

As shown in the previous section, it is possible to find an ion channel model that explains voltage-clamp data well and gives insight into the biophysical mechanism of the channel. However, in an excitable cell, there are many ion channel types that work together to generate specific patterns of firing activity. A cell is a complex system where multiple components interact nonlinearly (Koch and Segev, 2000). In contrast, voltage-clamp experiments isolate the channel of interest from this system and test it with predefined voltage waveforms. It is quite possible that some features of the kinetic mechanism that are critically important to the function of the cell may not be revealed in the voltageclamp data and may not be captured by the model. Ideally, the model should also be tested functionally, in a cellular context. 
A powerful tool for studying the function of voltage-gated ion channels in live neurons is dynamic-clamp (Tan and Joyner, 1990; Robinson and Kawai, 1993; Sharp et al., 1993). The principle is to pharmacologically block the channel of interest, and then functionally replace it with an injected current, dynamically calculated on the basis of a kinetic model (Milescu et al., 2008). As a first-order approximation, where we ignore the potential regulatory function of the permeant ions, $\mathrm{Ca}^{2+}$ in particular (Kaczmarek, 2006), the neuron makes no distinction between the native current and the model-based current, which are not necessarily carried by the same ions. Then, if the model is accurate, the neuron would exhibit the same firing pattern as with the actual channel. The sensitivity of the firing pattern to channel properties and the contribution of that particular current to spiking can be easily studied by varying the properties of the model and manipulating the model-based current in real-time. The major advantage of this hybrid experimentalcomputational approach is that a channel can be investigated within a live cell without any knowledge about other conductances or cell properties (Fig. 1.4).

\subsubsection{Solving the model in real-time}

Dynamic-clamp can be understood within the context of a cellular model. To make it easier to explain the concepts, we make several simplifying assumptions: $i$ ) besides voltage-independent leak channels, the neuron contains only Nav and Kv channels, with kinetic mechanisms described by Markov models; ii) the neuron has a single compartment and the membrane is isopotential; and iii) the model corresponds to an ideal whole-cell recording (zero access resistance, no pipette capacitance, etc.) (Marty and Neher, 1995). The state of the model as a function of time is completely described by 
three variables: the membrane potential $V$, and the state occupancies of the $\mathrm{Nav}$ and $\mathrm{Kv}$ channels, $\mathbf{P}_{\mathrm{Na}}$ and $\mathbf{P}_{\mathrm{K}}$. These state variables evolve in time according to the following ordinary differential equations:

$$
\begin{aligned}
& \text { (30) } C \frac{d V}{d t}=-\left(I_{\mathrm{Na}}+I_{K}+I_{\text {leak }}\right)+I_{\mathrm{app}}, \\
& \text { (31) } \frac{d \boldsymbol{P}_{K}}{d t}=\boldsymbol{P}_{K} \times \boldsymbol{Q}_{K}, \\
& \text { (32) } \frac{d \boldsymbol{P}_{\mathrm{Na}}}{d t}=\boldsymbol{P}_{\mathrm{Na}} \times \boldsymbol{Q}_{\mathrm{Na}},
\end{aligned}
$$

where $I_{\text {app }}$ is the current injected into the neuron through the patch-clamp pipette. To run a computer simulation of our model neuron, we would have to integrate these equations with an ODE solver. A real neuron "integrates" a similar set of differential equations, just more complex, to account for multiple cellular compartments, ion channel stochasticity, etc.

In voltage-clamp, $I_{\text {app }}$ is in principle equal to the sum of all ionic currents, so as to keep $V$ equal to the command voltage and $\mathrm{d} V / \mathrm{d} t$ equal to a predefined value (e.g., zero for a constant voltage step, or a finite value for a voltage ramp). In this sense, $I_{\text {app }}$ becomes a measure of the total ionic currents active in the cell. In current-clamp, $I_{\text {app }}$ is typically used to test the firing properties of a neuron under a range of conditions. For example, $I_{\text {app }}$ can be a constant value to bias the membrane potential, or it can be a predefined waveform that mimics excitatory or inhibitory synaptic input. In dynamic-clamp, $I_{\text {app }}$ is not predefined. Instead, $I_{\text {app }}$ is calculated in real-time, as a function of the membrane potential $V$ and some other quantities. 
How can dynamic-clamp be used to test a voltage-gated ion channel model in a live neuron? Let's consider the case of Nav channels. First, we pharmacologically block the channel, with TTX in this case. As the Nav current was eliminated, the equations "integrated" by the cell simplify to just two:

$$
\begin{aligned}
& C \frac{d V}{d t}=-\left(I_{K}+I_{\mathrm{leak}}\right)+I_{\mathrm{app}} \\
& \frac{d \boldsymbol{P}_{K}}{d t}=\boldsymbol{P}_{K} \times \boldsymbol{Q}_{K}
\end{aligned}
$$

Next, we replace the blocked current with a current generated by a Nav model that is solved on the computer. Effectively, we now have a hybrid biological-computational model that has the same set of ODEs, but two equations are "integrated" by the cell, and one is integrated on the computer:

$$
\begin{array}{ll}
C \frac{d V}{d t}=-\left(I_{K}+I_{\text {leak }}\right)+I_{\mathrm{app}}, & \text { "integrated" by the neuron } \\
\frac{d \boldsymbol{P}_{K}}{d t}=\boldsymbol{P}_{K} \times \boldsymbol{Q}_{K}, & \text { "integrated" by the neuron } \\
\frac{d \boldsymbol{P}_{\mathrm{Na}}}{d t}=\boldsymbol{P}_{\mathrm{Na}} \times \boldsymbol{Q}_{\mathrm{Na}}, & \text { integrated on the computer }
\end{array}
$$

where the injected current, $I_{\text {app}}$, is now equal to the negative current generated by the Nav model, $-\boldsymbol{I}_{\mathrm{Na}}^{\boldsymbol{C}}$, plus a constant component, $I_{\mathrm{inj}}$, that can be used to apply current steps or for other functions:

$$
I_{\mathrm{app}}=-I_{\mathrm{Na}}^{C}+I_{\text {inj }}
$$

The channel model is solved on the computer over discrete time steps, using the recursive method:

$$
\boldsymbol{P}_{\mathrm{Na}, \mathrm{t}+\delta t}=\boldsymbol{P}_{\mathrm{Na}, \mathrm{t}} \times \boldsymbol{A}_{\mathrm{Na}, \mathrm{V}},
$$


where $\mathrm{A}_{\mathrm{Na}, \mathrm{V}}$ is the transition probability matrix calculated for a given membrane potential $V$. $\mathbf{A}_{\mathrm{Na}, \mathrm{V}}$ can be pre-calculated over a voltage range (e.g., -100 to $+100 \mathrm{mV}$, every 0.1 $\mathrm{mV}$ ) and stored in a look-up table. As illustrated in Fig. 1.4, the model is solved in a realtime computational loop, where every iteration corresponds to one integration step. For each iteration, $V$ is read from the amplifier through the digital acquisition card (DAQ). Depending on $V$, the appropriate A matrix is selected from the look-up table and used to update $\boldsymbol{P}_{\mathrm{Na}}$. Then, $\boldsymbol{I}_{\mathrm{Na}}^{\boldsymbol{C}}$ is recalculated and injected into the neuron via $I_{\text {app. }}$. This loop must execute fast enough so that the voltage at the membrane does not change significantly within one iteration, which would invalidate both the selected $\mathbf{A}_{\mathrm{Na}, \mathrm{V}}$ and the injected current $\boldsymbol{I}_{\mathrm{Na}}^{C}$. The update rate should match the maximum rate of voltage change, which is typically the rising phase of the action potential. An update every $20 \mu \mathrm{s}(50 \mathrm{kHz})$, or faster, is generally adequate. Once an update interval is chosen, every iteration of the loop should be completed precisely within that time. To ensure predictable time steps with minimum variability, the code should run with real-time priority on the computer.

\section{$\underline{1.6 .2 ~ E q u i p m e n t ~ a n d ~ s o f t w a r e ~}$}

While dynamic-clamp can be performed under a variety of electrophysiology paradigms, we focus here on whole-cell patch-clamp experiments in neurons (Marty and Neher, 1995). Thus, in addition to the equipment and software used for patch-clamp, one also needs a digital acquisition card, a dedicated computer, and specialized software for realtime computation. Ideally, the patch-clamp amplifier should feature "true" current clamp. We had good results with HEKA's EPC 9 and 10 amplifiers, as well as with Molecular Devices' Multiclamp 700B. EPC 10 is more convenient, because it allows summation of 
external and internal current in current-clamp mode. In contrast, the Multiclamp amplifier has only one input connection for applied current, which is normally used by the external DigiData digitizer. A solution in this case is to use an electronic summation circuit or a mechanical switch box. Although we don't have first-hand experience with other instruments, there are several commercially available patch-clamp amplifiers that feature true current clamp, e.g., those made by A-M Systems, NPI, Warner Instruments, etc. Although some patch-clamp amplifiers already include an internal (EPC 10) or external (Multiclamp 700B) digitizer, these are not necessarily optimized for real-time feedback acquisition, where, in a very short time (tens of microseconds), a sample is read from the analog input, processed on the CPU, and another sample is written to the analog output. With a few exceptions (e.g., the hardware-based dynamic-clamp device commercialized by Cambridge Electronic Design), all dynamic-clamp applications use digitizers made by National Instruments. At the time of writing, we recommend the NI PCIe-6351 or NI PCIe-6361 (slightly faster) boards, which have been optimized for very low latency. One should be aware that the manufacturer typically specifies the maximum rate for buffered acquisition, not for real-time applications. Transferring one single sample across the PCle bus has a finite latency that limits dramatically the throughput rate in real-time acquisition. For example, the maximum rate that we could obtain with a NI PCIe-6361 card is $\sim 220,000$ computational cycles per second, even though the board can acquire 2 million samples per second in buffered mode. Nevertheless, a throughput rate like this is excellent, being comparable with the bandwidth of the patch-clamp amplifier in currentclamp. 
Historically, the first dynamic-clamp programs were coded in some flavor of real-time Linux (Christini et al., 1999; Butera Jr et al., 2001; Dorval et al., 2001). At the time, obtaining acceptable real-time performance under the Microsoft (MS) Windows operating system - or any other non-real-time OS - was simply not possible. On such a system, user programs can be interrupted at random by other programs, or by the operating system itself. Another limitation at the time was the driver provided by National Instruments for programming their boards, which was incredibly slow for realtime applications ( 1,000 cycles per second, according to our tests). However, the situation has completely changed over the last ten years, with the development by National Instruments of new digitizers and optimized drivers, with the advent of multicore processors and an improved PCIe bus, and with the general increase in CPU speeds. Today, dynamic-clamp software can be run in MS Windows with excellent real-time performance, on par with what is achieved under real-time Linux.

Dynamic-clamp programs are available for both real-time Linux (Lin et al., 2010) and MS Windows (Kullmann et al., 2004; Nowotny et al., 2006; Milescu et al., 2008; Yang et al., 2015). For the more biophysically-inclined user, we recommend our own implementation of dynamic-clamp in the QuB software, which runs under MS Windows (www.milesculabs.biology.missouri.edu/QuB). The major advantages are integration with a variety of ion channel modeling algorithms, a powerful scripting language for customized models and protocols, and sophisticated methods for solving Markov models of ion channels, deterministically or stochastically. QuB is also useful as it uses a series of parameters that can be modified in real-time without having to stop the script, 
recompile and restart the script. The graphic user interface is shown in Fig. 1.5. The only method that can be used to solve large Markov models accurately is the matrix method described in this chapter, which is available in our software. Once a few quantities are pre-calculated and stored in look-up tables, very large Markov models can be solved using only vector - matrix multiplications, which can be executed very quickly on modern CPUs or on graphics processors (GPUs). For example, we were able to run models with as many as 26 states at $50 \mathrm{kHz}$ or faster (Milescu et al., 2008). The matrix method is also very stable and accurate, even with long sampling intervals, which is generally not the case with methods that rely exclusively on ODE solvers to advance the state probabilities. In particular, integration with the Euler method, which is practically the only one that is fast enough for real-time applications, is bound to fail with even small Markov models (Milescu et al., 2008).

In principle, any desktop computer can be used for dynamic-clamp. However, for highperformance applications (large models and high throughput rates), we recommend a fast computer that is used exclusively to run the dynamic-clamp engine. We had the best results with multicore Intel Xeon CPUs, installed in dual-processor server-grade systems. For example, the computer that we currently use in the lab has two Intel Xeon E5-2667 v2 8-core processors, clocking at $3.3 \mathrm{GHz}$, and runs Windows 7 Pro 64-bit. Our system was built by ASL, Inc., but many other computer integrators sell configurable systems. Of all the components, the most critical are the CPU and the motherboard. 


\subsubsection{Preparing and running a dynamic clamp experiment}

Setting up a dynamic-clamp experiment involves a few steps. First, the voltage monitor output of the patch-clamp amplifier should be connected to one of the analog inputs of the National Instruments digitizer, while the external current input of the amplifier should be connected to one of the analog outputs of the digitizer. Next, one needs to zero the offsets and calibrate the scaling factors between the amplifier and digitizer, for both input and output. The calibration procedure depends on the specific dynamic-clamp software but the idea is to make sure that the membrane voltage value read into the dynamic-clamp software is the same as the value reported by the patch-clamp amplifier. Likewise, the external current reported by the amplifier should match the current sent by the dynamicclamp software. In our experience, there are always slight offsets of a few $\mathrm{mV}$ in membrane potential and a few $\mathrm{pA}$ in injected current, between the amplifier and the digitizer. These offsets must be compensated for in the software. In particular, one should be careful that the amplifier receives no unwanted external current when $I_{\text {app }}$ is equal to zero, as even a small current of a few pA can alter the firing pattern of a neuron. Most amplifiers have adjustable gain in current-clamp (e.g., $1 \mathrm{pA} / \mathrm{mV}$ ). The smallest gain should be selected that still allows the injection of the largest current that might be predicted by the model. For example, a model-based Nav current ranges from a few pA in the interspike interval, small but sufficient to influence neuronal firing properties, to several nA during an action potential.

The pipette capacitance should be reduced as much as possible by coating with Sylgard or other agents. In our experience, the residual capacitance should be no more than $5-6$ 
$\mathrm{pF}$, otherwise ringing may occur in dynamic-clamp when large currents are injected, particularly with Nav currents during action potentials. Once a patch is obtained, the typical artifact estimation and compensation procedures should be applied for series resistance and pipette capacitance, as well as for membrane capacitance. Then, upon switching to current-clamp, the pipette capacitance compensation should be slightly reduced $(10-20 \%)$ to avoid ringing, while series resistance should be compensated $100 \%$.

An example of a dynamic-clamp experiment is shown in Fig. 1.6, adapted from a study on excitatory pre-Bötzinger (pre-BötC) neurons in the brainstem respiratory slice (Yamanishi et al., 2018). There, the persistent $\mathrm{Na}^{+}$current $\left(\mathrm{I}_{\mathrm{NaP}}\right)$ was investigated with voltage-clamp protocols designed to characterize its properties of activation and inactivation, recovery from inactivation, and slow inactivation. The data obtained with these protocols were used to construct a state model and to estimate its kinetic parameters. Then, this model was tested in dynamic clamp experiments, to determine how $\mathrm{I}_{\mathrm{NaP}}$ contributes to bursting behavior. The pre-BötC circuit contains a mixture of both bursters and non-bursters, which differ in the total amount of $\mathrm{I}_{\mathrm{NaP}}$ (Koizumi et al., 2013). Since there is no selective blocker for $I_{\mathrm{NaP}}$, the $\mathrm{I}_{\mathrm{NaP}}$ model was either added to nonbursters (Fig.1.6B, 1.6C) or subtracted from intrinsic bursters (Fig. 1.6E). The results showed that injecting $\mathrm{I}_{\mathrm{NaP}}$ into non-bursters was sufficient to convert them to burst firing, exhibiting the characteristic burst after-hyperpolarization (bAHP) (Fig. 1.6B bottom) and modulation of bursting frequency by bias current injection (Fig. 1.6C). In contrast, subtracting $\mathrm{I}_{\mathrm{NaP}}$ from intrinsic bursters converted them to quiescence or tonic firing. 
Clearly, this $\mathrm{I}_{\mathrm{NaP}}$-based interconversion between bursting and non-bursting firing modes depends on the entire complement of ion channels expressed by pre-BötC neurons. For example, adding $\mathrm{I}_{\mathrm{NaP}}$ into other types of neurons, such as tonically firing ventral raphé neurons, cannot induce bursting.

\subsection{Conclusions}

We described here the overall process by which a kinetic model of a voltage-gated ion channel is constructed from experimental data. To do so, one needs a good understanding of data properties, including the experimental or recording artifacts that may be contaminating the signal. A variety of voltage-clamp protocols are necessary to make the channel visit as many conformations as possible, to shed light on the number of conformational state and the transitions between them. Furthermore, one should take into account prior knowledge from other studies and create a model that is in agreement with other types of data, as detailed in the next chapter. Finally, it is important to test the model with dynamic clamp, to verify that it can reproduce certain behaviors and thus confirm its accuracy. 


\subsection{Figures and figure legends}

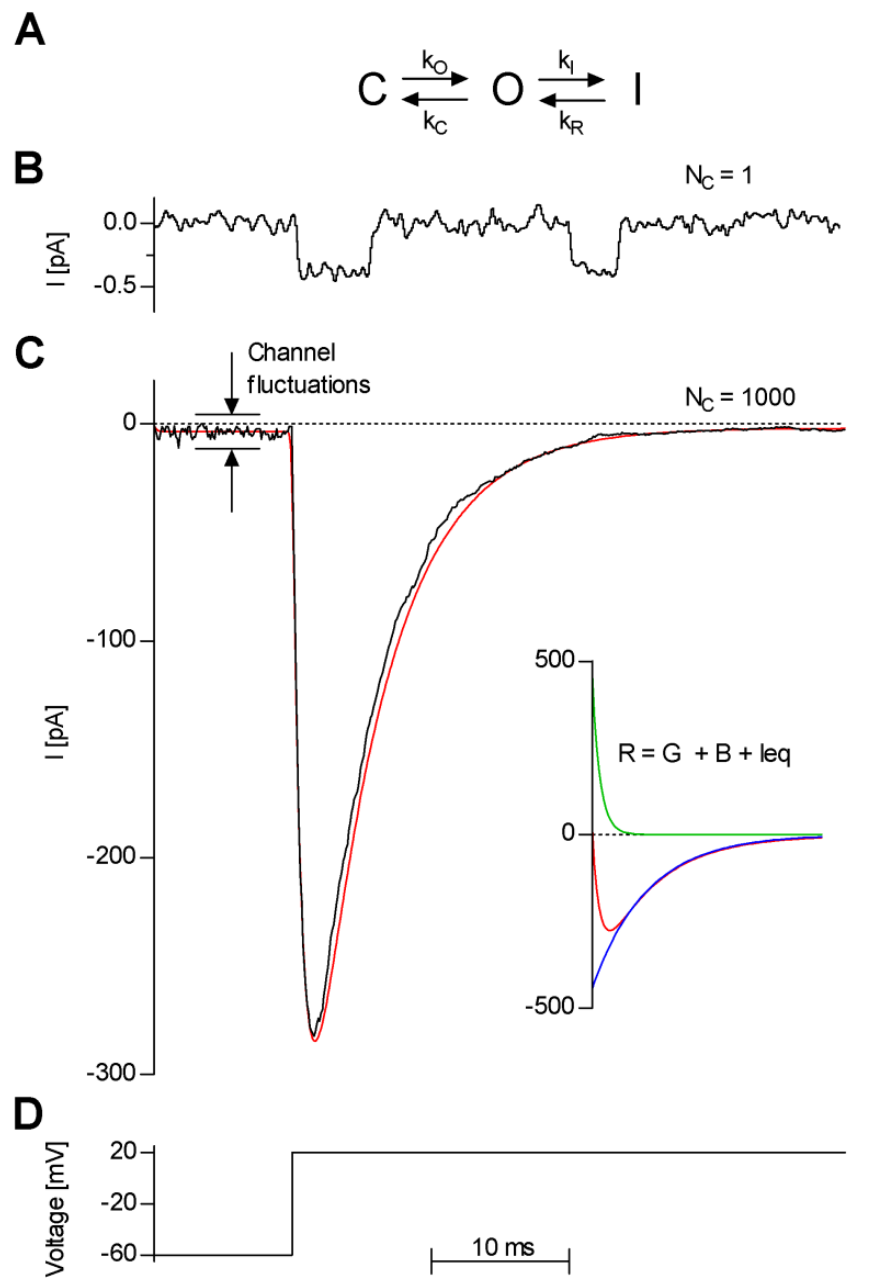

Figure 1.1. From model to data. A simple ion channel model (A) was used to simulate singlechannel (B) and macroscopic $(\mathbf{C})$ currents in response to a voltage step $(\mathbf{D})$. The macroscopic current was simulated with an ensemble of 1000 channels, either deterministically (black trace) or stochastically (red trace). The inset shows a fit of the stochastic macroscopic current (red) with a two-exponential function. The individual exponential components of the fit line are also shown (green and blue). 

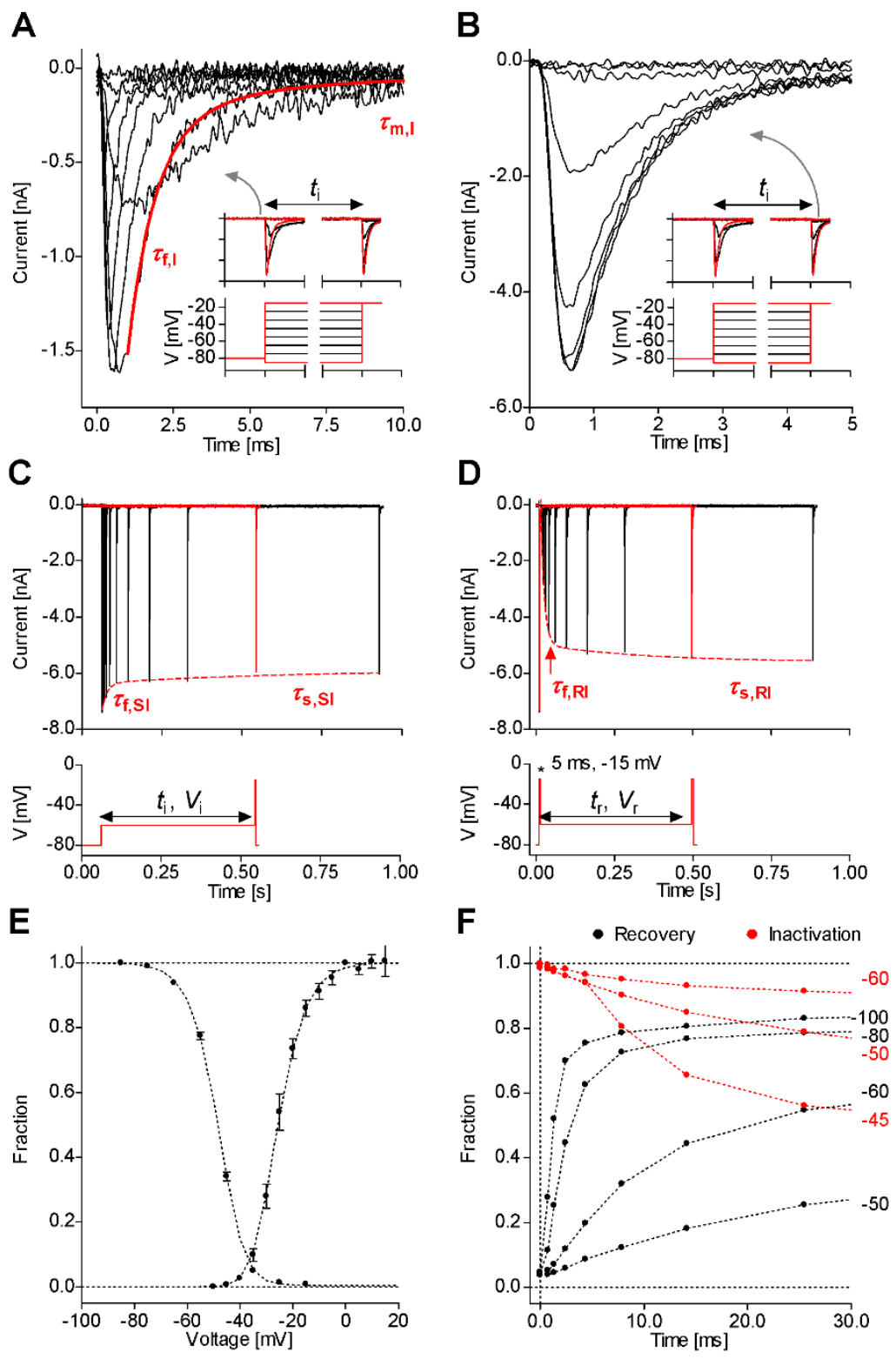

Figure 1.2. Designing voltage-clamp protocols for $\mathrm{Na}^{+}$currents. To gather information about the kinetic mechanism, the channels are forced to make transitions between different sets of states, as follows: deactivated to open to inactivated (A), deactivated to inactivated to open $(\mathbf{B})$, non-inactivated to inactivated $(\mathbf{C})$, and inactivated to non-inactivated (D). Raw data are further processed to extract state occupancies as a function of time and voltage $(\mathbf{E}$ and $\mathbf{F})$. Adapted from (Milescu et al 2010b) 
A

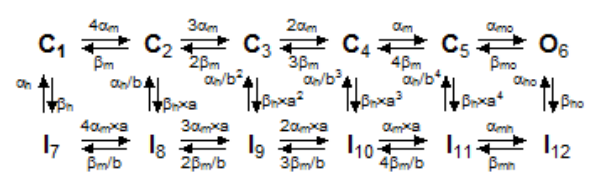

B

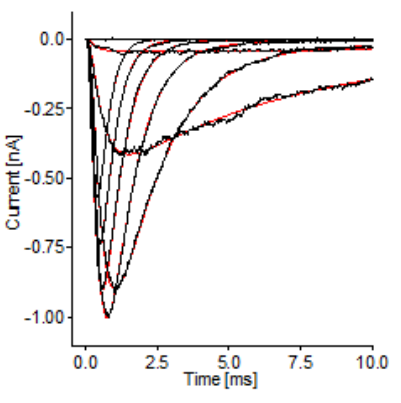

C

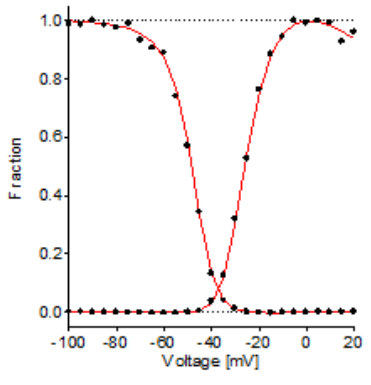

D

E
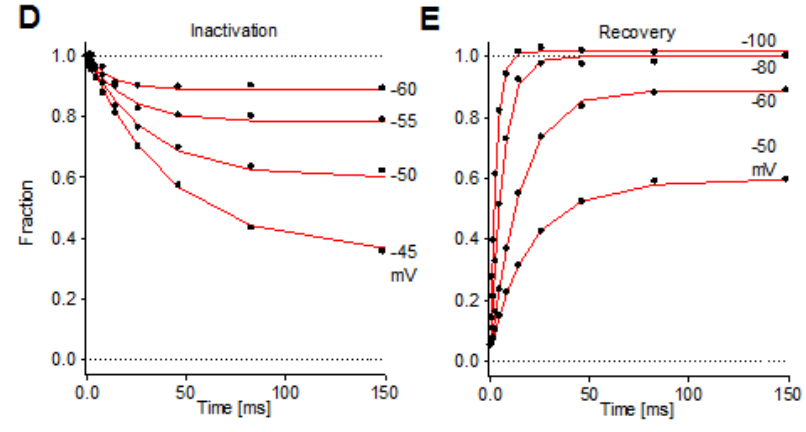

Figure 1.3. Fitting the data with a model. A) Model originally formulated for Nav channels (Kuo and Bean 1994). States $\mathrm{C}_{1} \ldots \mathrm{C}_{5}$ and $\mathrm{O}_{6}$ represent the non-inactivated channel, and $\mathrm{I}_{7} \ldots \mathrm{I}_{12}$ are inactivated states. $\mathrm{O}_{6}$ is the only conducting (open) state. The pathway from either $\mathrm{C}_{1}$ to $\mathrm{C}_{5}$, or from $\mathrm{I}_{7}$ to $\mathrm{I}_{12}$, corresponds to the activation of the four voltage sensors, assumed to be equal and independent. This assumption is denoted by the $4: 3: 2: 1$ or $1: 2: 3: 4$ ratios in the factors multiplying the $\alpha_{\mathrm{m}}$ or $\beta_{\mathrm{m}}$ rates. The $\mathrm{C}_{5}$ to $\mathrm{O}_{6}$ transition corresponds to the opening of the channel. The model allows the channel to inactivate without opening, from any of the closed states $\mathrm{C}_{1} \ldots \mathrm{C}_{5}$. However, the channel is most likely to inactivate from the open state $\mathrm{O}_{6}$, or when more voltage sensors are activated (e.g., from $\mathrm{C}_{5}$ ). B-E) Example fits of the model in A to the macroscopic data in Fig. 3. The data were pooled together and fitted with the kinetic mechanism shown in Fig4A, using a computational algorithm that minimized the sum of squared errors. The response of the model to the same voltage-clamp protocols as used to record the data is represented by the red trace, which corresponds to the best parameters found by the optimizer. B) Time course of activation. C) Voltage dependence of activation and inactivation. D) Subthreshold inactivation. E) Recovery from inactivation. 


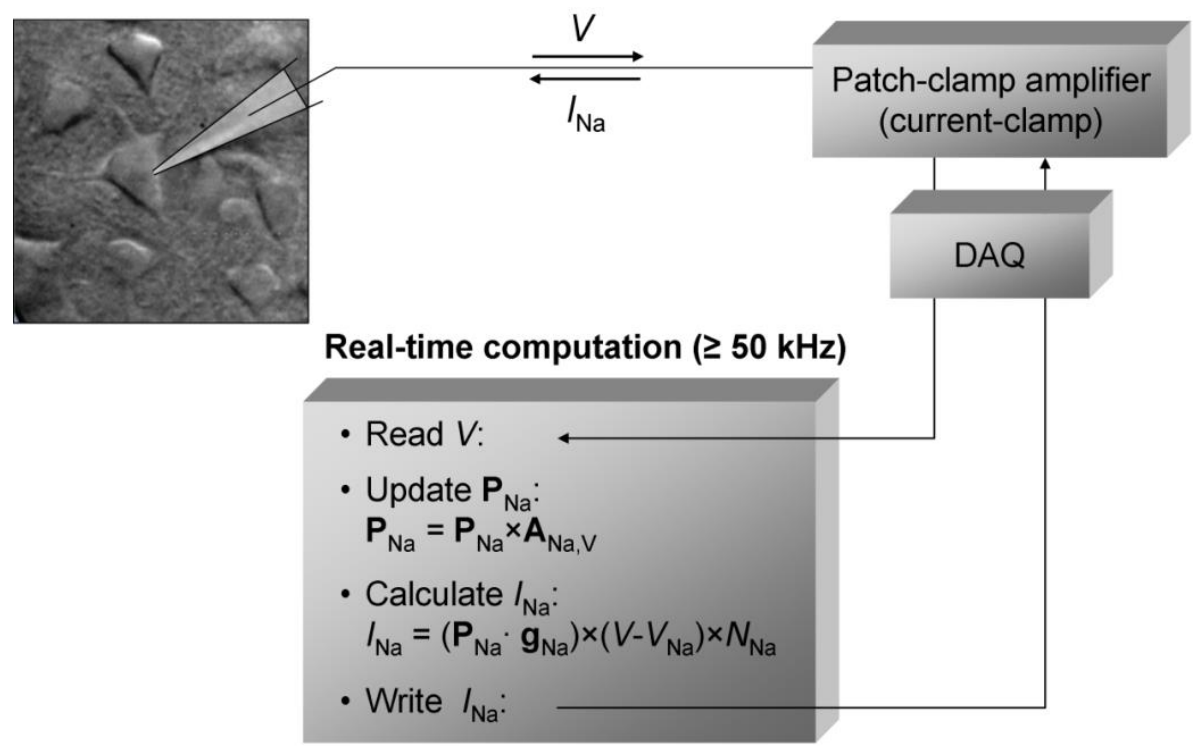

Figure 1.4. Testing ion channel models in live neurons with dynamic-clamp. As illustrated here, a Nav current is blocked with TTX and replaced with a model-based current, which is calculated in real-time on the basis of a model and injected into the neuron via the patch-clamp pipette. 


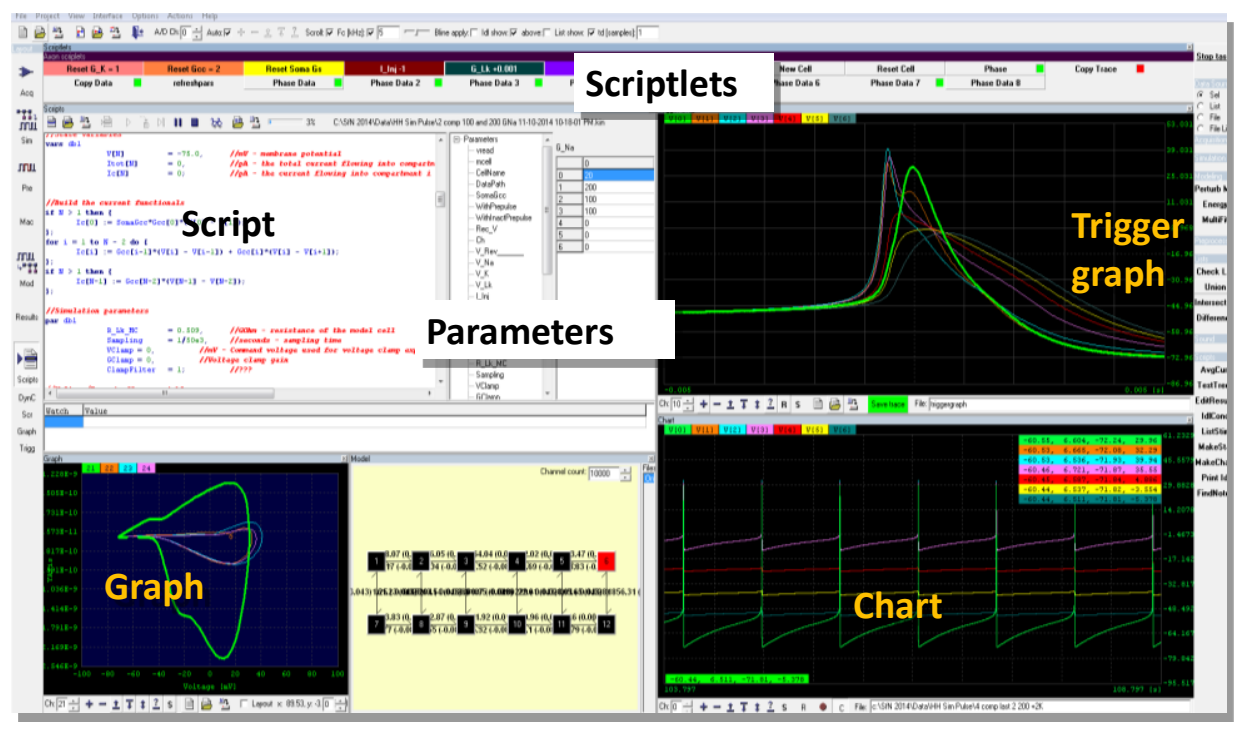

Figure 1.5 Running dynamic clamp in QuB. The screenshot is an example of how QuB can be set up for dynamic clamp experiments. The Script window includes a list of parameters of the model that can be readily changed and take effect in real time. This is useful to modify conductance values of particular current. Scriptlets are scripts that can run independently of the main script, and can be used to run a protocol or perform data analysis with a single mouse click. The Chart window displays the real-time acquisition of the data. The Trigger graph window is used to detect and display action potentials in real-time. The Graph window is used to display analyzed data, such as the phase plane of the AP, as shown here. 


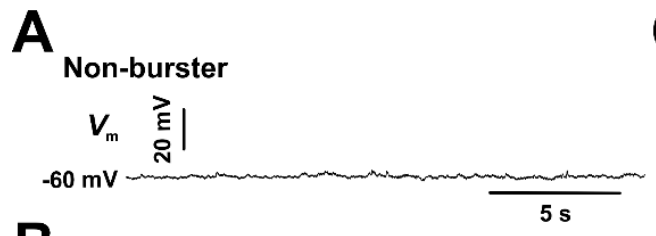

B

Non-burster / Model-1 (dynamic clamp) $\mathrm{G}_{\mathrm{NaP}}=1.0 \mathrm{nS}$

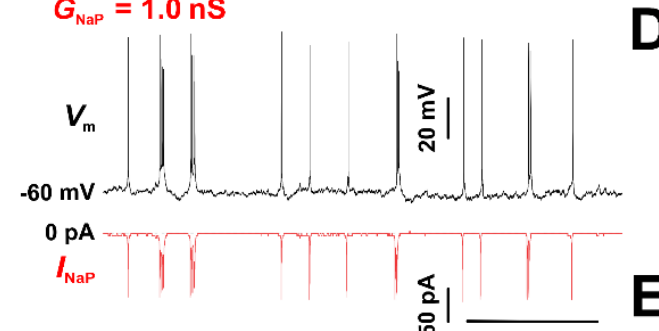

$G_{\mathrm{NaP}}=1.3 \mathrm{nS}$

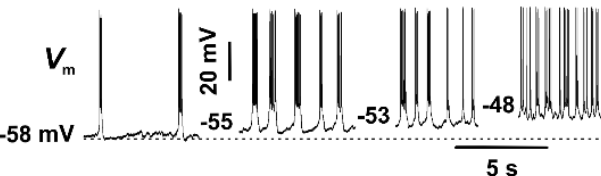

D
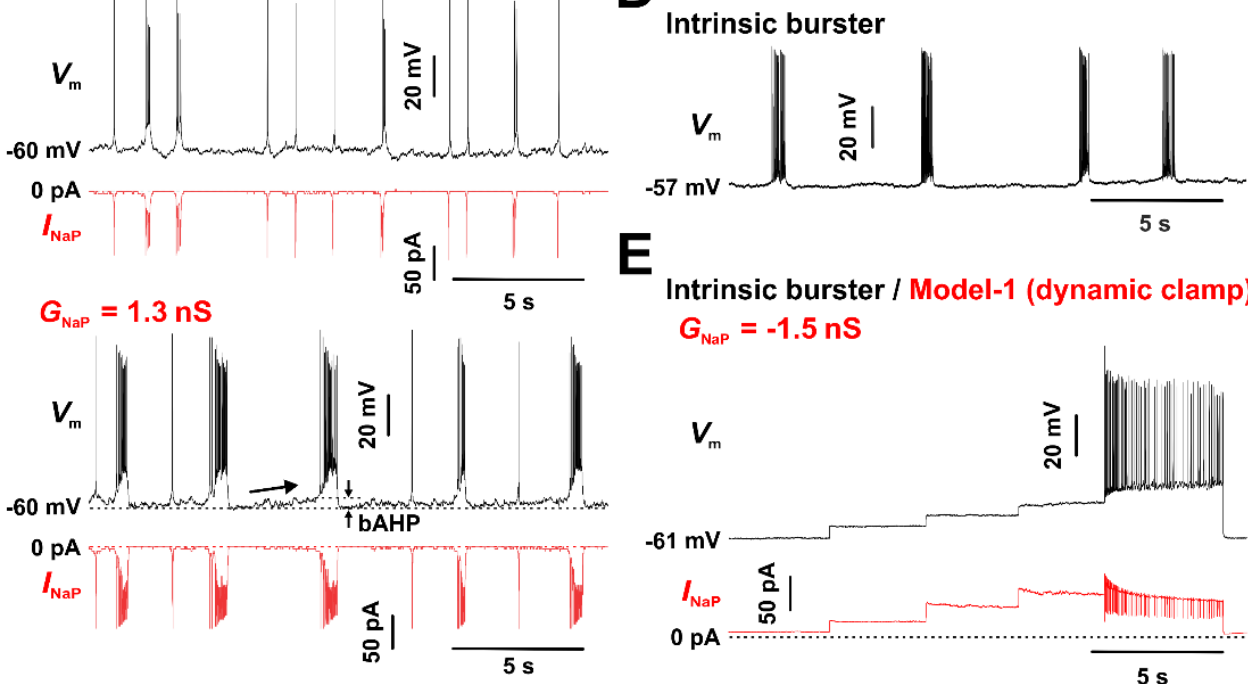

Intrinsic burster / Model-1 (dynamic clamp) $G_{\mathrm{NaP}}=-1.5 \mathrm{nS}$

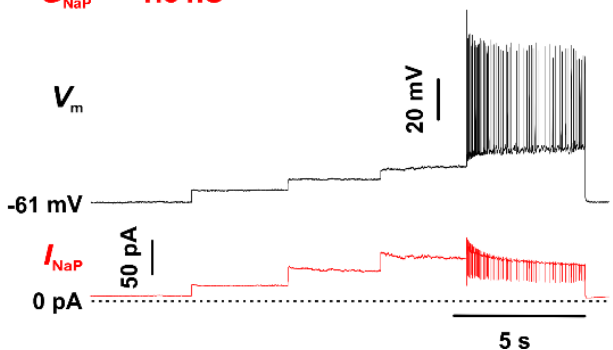

Figure 1.6. Dynamic clamp example. A novel Markov model was fit to voltage-clamp data of the $\mathrm{I}_{\mathrm{NaP}}$ in pre-BötC neurons. $\mathrm{I}_{\mathrm{NaP}}$ predicted by Model 1 was added to nonbursters $\left(\mathrm{G}_{\mathrm{NaP}}>0\right)$ or subtracted from bursters $\left(\mathrm{G}_{\mathrm{NaP}}<0\right)$ via dynamic clamp. The recordings were obtained under bathapplied $\mathrm{Cd}^{2+}(200 \mu \mathrm{M})$ and CNQX $(20 \mu \mathrm{M})$. A) Current clamp (CC) recording from a representative nonburster. The neuron was verified to fire repetitively when sufficiently depolarized by injecting bias current (not depicted). B) Same cell as in A, but with $\mathrm{I}_{\mathrm{NaP}}$ added. When sufficient $\mathrm{I}_{\mathrm{NaP}}$ is injected $\left(\mathrm{G}_{\mathrm{NaP}}=1.3 \mathrm{nS}\right)$, the neuron exhibits robust bursting, with the back after hyperpolarization (bAHP) and the interburst depolarization drift (arrow) characteristic of intrinsic bursters. C) Bursting frequency depends on baseline membrane potential (modified with depolarizing bias current), a behavior typical of intrinsic bursters. D) CC recording from a representative intrinsic burster. E) Same cell as in $\mathrm{D}$, but with $\mathrm{I}_{\mathrm{NaP}}$ subtracted. The step changes in membrane potential $\mathrm{V}_{\mathrm{m}}$ and $\mathrm{I}_{\mathrm{NaP}}$ reflect depolarizing bias current steps of 5-10 pA. $\mathrm{I}_{\mathrm{NaP}}$ subtraction eliminates the ability to generate bursting activity, but the neuron can fire repetitively when sufficiently depolarized. 


\title{
Chapter 2
}

\section{Estimating kinetic mechanisms with prior knowledge: linear and nonlinear parameter constraints}

This chapter has been adapted from:

Salari A.*, M.A. Navarro*, M. Milescu, L.S. Milescu. 2018. Estimating kinetic mechanisms with prior knowledge I: Linear parameter constraints. J. Gen. Physiol., 150(2):323-338. doi: 10.1085/jgp.201711912

Navarro, M.A.*, A. Salari*, M. Milescu, L.S. Milescu. 2018. Estimating kinetic mechanisms with prior knowledge II: Behavioral constraints and numerical tests. J. Gen. Physiol., 150(2):339-354. doi: 10.1085/jgp.201711913

*equal authorship

\begin{abstract}
Kinetic mechanisms predict how ion channels and other proteins function at the molecular and cellular levels. Algorithms have been developed that can be used to extract kinetic parameters from a variety of experimental data types. Ideally, a kinetic model should explain new data but also be consistent with existing knowledge. This chapter describes mathematical and computational formalisms that can be used to enforce prior knowledge into a model using constraints. These constraints can enforce explicit linear relationships involving rate constants, as well as nonlinear relationships that can quantify
\end{abstract}


a behavior of the model that cannot be easily expressed through mathematical relationships. For linear constraints we develop a formalism based on a linear algebra transformation, and for the other types we develop a penalty estimation mechanism. The linear transformation converts the set of linearly-interdependent model parameters into a reduced set of independent parameters, which can be passed to an automated search engine for model optimization. These can be applied to enforce many types of model properties and assumptions, such as microscopic reversibility, allosteric gating, equality and inequality parameter relationships. Examples of nonlinear properties that require nonlinear or behavioral constraints include the maximum open probability, use-dependent availability, and nonlinear parameter relationships. In principle, these procedures can be coupled to any of the existing methods for solving molecular kinetics - for ion channels or other proteins. By incorporating more knowledge into the parameter estimation procedure, it is possible to obtain more realistic and robust models, with greater predictive power.

Author contribution: M.A.N. designed scripts to test and implement the algorithms to fit sets of experimental data, implemented behavioral constraints, tested the penalty method in QuB, made figures, and wrote the manuscripts. 


\subsection{Introduction}

Ion channels are highly adapted to perform specific functions in the cell. Voltage-gated sodium (Nav) channels have finely tuned kinetic properties that allow neurons and other excitable cells to generate action potentials of specific shape and frequency (Bean, 2007). The properties that enable Nav and other channels to perform such complex and well calibrated behavior are encoded in the kinetic mechanism, defined as a set of conformational and functional states, interconnected by a network of allowed state transitions that may depend on ligand concentration, membrane potential, or other physical variables (Colquhoun and Hawkes, 1995a; Colquhoun and Hawkes, 1995b). Taking advantage of molecular biology to create protein truncations or point mutations have identified key domains of ion channels, such as the C-terminus (Wang et al., 2011a; Ben-Johny et al., 2014) and the voltage sensors (Armstrong and Bezanilla, 1977; Bezanilla and Armstrong, 1977; Stühmer et al., 1989). The activity and movement of these domains are essential to overall function of the protein and cells; therefore, the kinetic mechanism must be decrypted.

Critical advancements in laser photonics (Strickland and Mourou, 1985) have helped produce the optical trap (Ashkin, 1970; Ashkin et al., 1986), which has provided means to resolve tiny conformational changes of single-molecules (Smith et al., 1992; Svoboda, 1993). The data produced by single-molecule techniques, such as patch clamp electrophysiology (Sigworth, 1980b), FRET (Forrester Resonance Energy Transfer) (Ben-Johny et al., 2013) and optical tweezers (Bustamante et al., 2000), are often 
recordings over time with the desired signal of multiple events found with an underlying noise.

A typical approach to kinetic modeling is to fit experimental data with a memory-less Markov model, characterized by rate constants. Markov models have been used to analyze many types of data in biology, from single-channel (Qin, 1996, 2000a; Popescu and Auerbach, 2003) and macroscopic recordings of ligand-gated receptors or voltagegated ion channels, the catalytic rate of ribosomes (Kaiser et al., 2011), and the walking rates of microtubule molecular motors myosin V (Yildiz et al., 2003; Syed et al., 2010) and kinesin (Yildiz et al., 2004; Milescu et al., 2006). Decades of ion channel research have shown that kinetic mechanisms cannot be fully captured by any single type of experiment and must include a variety of experimental protocols to force the proteins of interest into many data states.

To construct a good model that may be useful, one must fit a comprehensive data collection (Horn and Lange, 1983; Hawkes et al., 1990; Vandenberg and Bezanilla, 1991a; Hawkes et al., 1992; Hoshi et al., 1994; Zagotta et al., 1994a; Zagotta et al., 1994b; Schoppa and Sigworth, 1998a, b; Rothberg and Magleby, 2000; Milescu, 2005), ideally generated by multiple experimental paradigms (Vandenberg and Bezanilla, 1991b; Akk et al., 2005; Milescu et al., 2008). For example, we know that Nav and other channels have four voltage sensors that gate with different timing and voltage sensitivity (Bezanilla, 2000a; Chanda and Bezanilla, 2002b). These fundamental aspects cannot be easily resolved by single channel or whole-cell recordings alone, but they can be 
addressed in combination with other types of experiments, such as patch-clamp fluorometry (Chanda and Bezanilla, 2002a; Zaydman et al., 2013; Pantazis et al., 2014).

Optimizing a model against multiple types of data is a difficult problem in itself. A further complication is that some results - quantitative or qualitative - cannot be added to the data collection that is used for fitting. The number of voltage sensors, the existence of open-state block, numerical relationships between parameters due to allosterism, etc., are examples of such results. Instead, this prior knowledge about the channel must be encoded directly into the model. In this way, the model will explain the new data but will also remain consistent with what is already known.

How do we introduce prior knowledge into a model? We present here some strategies for addressing this issue. At the most basic level, structural assumptions about the kinetic mechanism can be stated implicitly by choosing a specific set of states and connectivity, as we explain with an example from the literature (Kuo and Bean, 1994). Further quantitative or qualitative assumptions can be introduced by defining a set of constraints that the model has to satisfy, while also explaining the new data. These model constraints can be formulated as explicit mathematical relationships between rate constants or other model parameters or can specify the behavior of the model under certain conditions (Fig.

\section{1).}

To develop new model constraints, an existing method for enforcing linear constraints between rate constants (Qin, 1996; Colquhoun, 2004; Milescu, 2005) was extended to cover arbitrary linear constraints between model parameters, including allosteric factors, reducing the parameter search space (Salari et al., 2018). This new formalism can handle 
both equality and inequality relationships allowing for direct modulation of the parameter search. The second half of this work describes a method to implement arbitrary parameter relationships, as well as behavioral constraints, by adding a penalty term to the cost function of the fitting algorithm (Navarro et al., 2018). The theory and computational procedures described here can be coupled, in principle, to any of the existing methods for solving molecular kinetics, for ion channels or other proteins. These concepts can be used not only to enforce existing knowledge, but also to formulate and test new hypotheses.

\subsection{Materials and methods}

All the mathematical and computational algorithms described in this study were implemented and tested with the freely available MLab edition of the QuB program (http://milesculabs.biology.missouri.edu/QuB), running under the Microsoft Windows operating system. QuB provides a suite of algorithms for extracting ion channel kinetic models and parameters from single molecule and macroscopic data (Qin et al., 1996, 2000a, b; Milescu, 2005), and for testing these models in live cells using real-time computation (dynamic clamp) (Milescu et al., 2008, Milescu et al., 2010b).

\subsubsection{Model parameters}

To simulate the test data, the model shown in Fig. 2.6A was tweaked by hand to generate macroscopic currents resembling voltage-dependent sodium currents (Fig. 2.7). The simulated data were fitted in multiple runs, with different sets of constraints applied to the model (Fig. 2.6B). The model parameter values (true, initial, and estimated) are listed in Table 2.1. 


\subsubsection{Stochastic simulations}

Ion channel macroscopic traces were simulated stochastically under the voltage-clamp paradigm, using established procedures (Milescu, 2005). To approximate the properties of sodium currents, the single channel conductance was $10 \mathrm{pS}$ and the reversal potential was $+60 \mathrm{mV}$. Random Gaussian noise with zero average and $5 \mathrm{pA}$ standard deviation was added to each trace, to approximate whole-cell recording noise. To generate activation / inactivation time course (Fig. 2.7A) and activation / availability steady-state curves (Fig. 2.7B), we used a typical voltage-clamp protocol: the channels were first equilibrated at $120 \mathrm{mV}$, then subjected to a $200 \mathrm{~ms}$ conditioning step at potentials ranging from $-120 \mathrm{mV}$ to $+40 \mathrm{mV}$, followed by a $50 \mathrm{~ms}$ test step at $0 \mathrm{mV}$. The peak current from each conditioning step was extracted and converted to conductance (assuming a linear relationship), and the obtained values were used to construct the activation curve. Similarly, the peak current from the test step was extracted and used to construct the availability curve. Together, the currents evoked during the first $5 \mathrm{~ms}$ of the conditioning step in the $-50 \mathrm{mV} \ldots+40 \mathrm{mV}$ range (Fig $\mathbf{2 . 7 A}$ ) and the activation and availability curves

(Fig 2.7B) were used for model optimization.

\section{$\underline{\text { 2.2.3 Model optimization }}$}

The algorithms were tested by fitting the data shown in Fig 2.7. Optimization trials were run on a dual eight-core $3.3 \mathrm{GHz}$ Intel Xeon processor computer, running Windows 7 64bit. Each optimization run took less than 20 minutes to complete. The model was optimized by minimizing the cost function with a modified version of the Davidon- 
Fletcher-Powell optimizer (dfpmin) (Fletcher and Powell, 1963; Press et al., 1992). For efficiency, the cost function was coded for parallel computation. The cost function was calculated as the sum of square errors between the data and the prediction of the model, normalized to the total number of points, plus a penalty term for those optimization trials involving a behavioral constraint, as detailed in section 2.5 . The gradients of the costfunction with respect to the free parameters were calculated numerically. The prediction of the model for a given set of parameters was obtained by simulating the deterministic response of the model to the same stimulation protocols as used for simulation. Then, the resulting traces were processed to extract the time course and the activation and availability curves, following the same procedure as for the simulated test data (Milescu et al., 2010b; Salari et al., 2016).

\subsection{Theoretical background}

\subsubsection{Kinetic mechanisms}

Markov models reduce the continuum of molecular conformations that can be assumed by the protein to a small set of discrete states that can be detected experimentally or inferred statistically (Colquhoun and Hawkes, 1995a; Colquhoun and Hawkes, 1995b; Colquhoun and Sigworth, 1995). These states correspond to various conformations of functional and structural elements, such as resting or activated voltage sensors, bound or unbound ligand, closed or open pore, inactivated or non-inactivated channel, etc. Direct transitions are permitted between certain states, and the frequency of these transitions is quantified by Eyring rate constants, which can be functions of ligand concentration, 
membrane potential, tension, or other physical variables. The topology (or structure) of a kinetic mechanism is defined by the set of states and their transition connectivity, including information on which rates are ligand-dependent, voltage-dependent, etc.

Here, we assume that all rate constants follow the Eyring formalism (Eq. 1) (Eyring, 1935a), with the implication that complexity in kinetic behavior should be explained with more elaborate state models, rather than through over-parameterized and ad hoc rate constant expressions. Accordingly, voltage-dependent rate constants are simple exponential functions of voltage:

$$
\text { (1) } \quad k_{\mathrm{ij}}=k_{\mathrm{ij}}^{0} \times e^{k_{\mathrm{ij}}^{1} \times V} \text {, }
$$

where $k_{\mathrm{ij}}$ is the rate constant of the transition from state $i$ to state $j$, and $V$ is the membrane potential. The $k_{\mathrm{ij}}^{0}$ value is the rate constant at zero membrane depolarization, while the $k_{\mathrm{ij}}^{1}$ value is the voltage sensitivity factor, which can be expanded as follows:

$$
\text { (2) } \quad k_{\mathrm{ij}}^{1}=\left(\delta_{\mathrm{ij}} \times z_{\mathrm{ij}} \times F\right) /(R \times T) \text {, }
$$

where $z_{\mathrm{ij}}$ is the electrical charge moving over the fraction $\delta_{\mathrm{ij}}$ of the electric field, $F$ is Faraday's constant, $R$ is the gas constant, and $T$ is the absolute temperature. For voltageinsensitive rates, $k_{\mathrm{ij}}^{1}=0$. Rate constants that depend on other physical variables, such as membrane tension, have similar exponential expressions (Gnanasambandam et al., 2017). For state transitions that represent the binding of a ligand, rate constants have the following expression:

(3) $\quad k_{\mathrm{ij}}=k_{\mathrm{ij}}^{0} \times[L]$, 
where $k_{\mathrm{ij}}^{0}$ is the rate constant at unitary ligand concentration $[L]$.

The set of $k_{\mathrm{ij}}^{0}$ and $k_{\mathrm{ij}}^{1}$ values are the main parameters of the kinetic mechanism. Together, the kinetic parameters and the topology of the model fully specify the mechanism. In turn, the kinetic mechanism describes the operation of the channel within the membrane, particularly how the channel changes its state in response to stimuli. Markov models, computational algorithms, and software have been adapted and developed to extract the kinetic mechanism from experimental data (Ball and Sansom, 1989; Hawkes et al., 1990; Qin, 1996, 2000a, b; Venkataramanan and Sigworth, 2002; Celentano and Hawkes, 2004; Qin and Li, 2004; Milescu, 2005; Csanady, 2006; Moffatt, 2007; Stepanyuk et al., 2011; Stepanyuk et al., 2014), with two interrelated aims: to find an appropriate topology and to estimate the kinetic parameters.

\subsubsection{Formulating the topology of the model}

The first step in building a kinetic model is to identify a particular topology that defines the structural and functional elements of the channel and their connecting pathways. The topology can be used to specify the number of voltage sensors, the identity of voltagesensitive transitions, the number of inactivated states, the presence of multiple open states, the existence of allosteric relationships, etc. The models shown in Fig. 2.2 illustrate how a topology can be formulated to capture the key features of a Nav channel kinetic mechanism, as detailed in section 2.5.5. This model was based on a large body of knowledge accumulated in the field and, not surprisingly, provided a flexible enough framework that explained voltage clamp recordings of sodium currents in several 
neuronal preparations (Kuo and Bean, 1994; Raman, 2001; Taddese and Bean, 2002; Milescu et al., 2010b).

In contrast, when little is known about the channel, one must take a purely data-driven approach and build a parsimonious topology that explains the data reasonably well. Of course, if one wishes a realistic model, then the Eyring theory and related concepts must still be obeyed. Some kinetic properties are intuitive enough and can be easily translated into model features (Salari et al., 2016). For example, whole-cell recordings where the current first rises and then decays require a model with one conducting and at least two non-conducting states. In general, searching for the right topology can be approached as an iterative process, where one tests a series of models of increasing complexity, adding more and more states and connections. For each tested model, one must determine whether the topology is compatible with the data. If no parameter values can be found that result in a good fit, the topology must be reformulated and parameters re-estimated. Since larger models can inherently fit better, one should take into account the number of degrees of freedom when ranking models. Thus, unless a larger model improves the fit substantially, one should give preference to a model with fewer free parameters. The search across topologies can be terminated when the fit no longer improves.

The model search process is not trivial and relies much on the experience of the investigator. The number of non-equivalent (Kienker, 1989) connectivity schemes can be prohibitively large, even for models with a relatively small state count (Bruno et al., 2005). A possible solution is to use a smart optimization algorithm that not only estimates parameters for a given topology but at the same time searches efficiently across 
topologies (Gurkiewicz, 2007; Menon et al., 2009). Furthermore, one may be able to reduce the searched state space by utilizing some information contained in the data. For example, statistical analysis of single channel electrical recordings through dwell time histogram analysis can provide reasonable estimates on the number of conductance levels (through visual inspection or amplitude histogram analysis) and the minimum number of kinetic states in each conductance level (Colquhoun and Hawkes, 1982; Hawkes et al., 1990). Other methods can provide more direct evidence about the structural conformations and transition pathways of the channel, such as the number of voltage sensors, the number of inactivated states, or the identity of voltage- or ligand-dependent transitions (Grosman et al., 2000; Ahern et al., 2016). In principle, an automated search across model topologies can incorporate this information (Teed and Silva, 2016).

\subsubsection{Parameter estimation}

A computational procedure for finding the "best" parameters for a proposed model topology combines an algorithm that measures how well a given model explains the data, with an optimization engine that searches the parameter space for the "best" solution (Fletcher, 2013). This optimal solution minimizes the error between the data and the prediction of the model (e.g., the sum of square errors), or maximizes a probability function (e.g., the likelihood that the experimental data were generated by the model, or the Bayesian posterior probability) (Horn and Lange, 1983; Hawkes et al., 1990; Qin, 1996, 2000a; Celentano and Hawkes, 2004; Milescu, 2005; Csanady, 2006; Moffatt, 2007; Calderhead et al., 2013; Stepanyuk et al., 2014; Epstein et al., 2016). Intuitively, the first approach can be described as minimizing a "cost function", while the second as 
maximizing a "goodness of fit". Throughout this study, we will use the "cost function" term, but with the understanding that it could refer to either minimizing the sum of square errors, or, equivalently, to minimizing the negative log-likelihood. When one also searches for an appropriate topology, the value of the cost function can be used as a score to rank the model. Even though the kinetic mechanism is fully characterized by the $k_{\mathrm{ij}}^{0}$ and $k_{\mathrm{ij}}^{1}$ parameters, the experimental data typically depend on some other parameters as well, such as the number of active ion channels, the single channel conductance, the ionic concentrations, etc. To extract the kinetic mechanism from the data, these other parameters may need to be co-estimated (Colquhoun, 1996; Qin, 2000b; Milescu, 2005).

\subsection{Results}

Once a model topology is selected to appropriately express what is known or hypothesized about the channel, the next step is to find a set of parameters that explain the data well. The parameter estimation procedure can be designed to enforce prior knowledge with the use of model constraints to generate a more selective set of parameter values, ideally reducing the total number of free parameters. These model constraints come in two categories: $i$ ) parameter constraints (Salari et al., 2018) and ii) behavioral constraints (Navarro et al., 2018). A parameter constraint is formulated as an explicit mathematical relationship that involves rate constants or other model parameters. An example is the scaling of one rate constant to another or restricting the range of a parameter to positive values. In contrast, a behavioral constraint quantifies the behavior of the model under certain conditions, without explicitly referring to rate constants or 
other model parameters. An example is enforcing the maximum open probability $\left(P_{\mathrm{O}}\right)$ reached by the channel during a specific voltage-clamp stimulation protocol.

The mathematical and computational procedures that reduce the number of free parameters to enforce parameter constraints are limited to linear relationships (Salari et al., 2018). However, nonlinear relationships can be enforced using the mechanism developed for behavioral constraints (Navarro et al., 2018). As illustrated in Fig. 2.1, linear parameter constraints that enforce an equality relationship reduce the dimensionality of the parameter space, eliminating one dimension for each relationship. In contrast, both inequality parameter constraints and behavioral constraints preserve dimensionality, but reduce the size of the parameter space. To describe it intuitively, inequality parameter constraints present the optimizer with a reduced road map, whereas behavioral constraints guide the optimizer through only toll-free roads.

\subsection{Implementing prior knowledge with linear parameter constraints}

In this section, we discuss the implementation of prior knowledge via linear parameter constraints using the Nav channel kinetic mechanism shown in Fig. 2.2A. This model is a good example, as it covers many of the parameter constraints that our formalism can enforce. Similar model topologies that have a combination of inactivation states have been proposed (Horn and Vandenberg, 1984; Benndorf, 1988), but the first model that incorporates evidence that inactivation can proceed from the closed state was developed with many mechanistic assumptions in mind (Kuo and Bean, 1994). These are reflected in the number of states and connections, and in the mathematical relationships between 
various kinetic parameters (Fig. 2.2B). These assumptions can be regarded and expressed as parameter constraints.

\subsubsection{Model assumptions}

The first assumption is that channel activation involves four identical and independent voltage sensors, and all four must be activated to open the pore. Of course, this assumption is not necessarily true, as more recent evidence obtained with voltage clamp fluorometry indicates that the channel can open when only three voltage sensors are activated (Capes et al., 2013; Pantazis et al., 2014; Goldschen-Ohm and Chanda, 2015). In Fig. 2.2A, all closed states with the same number of resting sensors $(n)$ are lumped into a single compound state. The result is the five-state activation pathway $\mathrm{C}_{1} \ldots \mathrm{C}_{5}$. The frequency of activation transitions for any of the compound states $\mathrm{C}_{1} \ldots \mathrm{C}_{5}$ is equal to $n$ times the frequency of the activation transition for a single sensor. The same rule applies to deactivation transitions. For example, when the channel resides in a closed state that has three resting voltage sensors $\left(\mathrm{C}_{2}, n=3\right)$, the compound activation rate $\left(k_{2,3}\right)$ is three times the activation rate of a single sensor $\left(k_{4,5}\right.$ or $\left.\alpha_{m}\right)$. Thus, if $k_{4,5}$ and $k_{2,1}$ are the transition rates of a single sensor activating or deactivating, respectively, the assumption of identical and independent voltage sensors is expressed by the following mathematical relationships, where one rate is scaled to another by a constant factor:

$$
\begin{aligned}
& k_{3,4}=2 \times k_{4,5}, \\
& k_{2,3}=3 \times k_{4,5}, \\
& k_{1,2}=4 \times k_{4,5},
\end{aligned}
$$




$$
\begin{aligned}
& k_{3,2}=2 \times k_{2,1}, \\
& k_{4,3}=3 \times k_{2,1}, \\
& k_{5,4}=4 \times k_{2,1} .
\end{aligned}
$$

Any deviation from the condition of identical and independent voltage sensors will require a model with a different number of states, connections, and multiplication factors along the activation pathway. In fact, these constant multiplication factors $(2,3$, and 4$)$ could be replaced with unknown cooperativity factors, to be determined from the experimental data, similar to the inactivation allosteric factors introduced next. The same principles apply to ligand-gated ion channel mechanisms. In this case, the multiplication factors (sometimes called "statistical" factors) are used to describe the relationships between the ligand binding sites.

Another assumption is that the channel can inactivate not only from the open state $\mathrm{O}_{6}$, but also from any of the $\mathrm{C}_{1} \ldots \mathrm{C}_{5}$ closed states into the $\mathrm{I}_{7} \ldots \mathrm{I}_{12}$ inactivated states. However, the transition into inactivation from the closed states depends on the degree of activation: as more voltage sensors are activated, the $\mathrm{C}$ to I transitions become faster, whereas the I to $\mathrm{C}$ become slower. As envisioned in the original model, this property is implemented with the allosteric factors $a$ and $b$. Thus, the rate of inactivation from a closed state $\mathrm{C}_{\mathrm{n}}$ is equal to the rate of inactivation from the previous closed state, $\mathrm{C}_{\mathrm{n}-1}$, multiplied by the $a$ factor. For example, $k_{2,8}=a \times k_{1,7}, k_{3,9}=a \times k_{2,8}$, etc. The opposite is true for the return rates: $k_{8,2}=b^{-1} \times k_{7,1}, k_{9,3}=b^{-1} \times k_{8,2}$, etc. Taking $k_{1,7}$ and $k_{7,1}$ as references, this assumption is expressed by the following mathematical relationships, where one rate is scaled to another by a variable factor: 


$$
\begin{aligned}
& k_{2,8}=a \times k_{1,7}, \\
& k_{3,9}=a^{2} \times k_{1,7}, \\
& k_{4,10}=a^{3} \times k_{1,7}, \\
& k_{5,11}=a^{4} \times k_{1,7}, \\
& k_{8,2}=b^{-1} \times k_{7,1}, \\
& k_{9,3}=b^{-2} \times k_{7,1}, \\
& k_{10,4}=b^{-3} \times k_{7,1}, \\
& k_{11,5}=b^{-4} \times k_{7,1} .
\end{aligned}
$$

Furthermore, the voltage sensors can also activate while the channel is inactivated, along the $\mathrm{I}_{7} \ldots \mathrm{I}_{11}$ pathway, but with different kinetics, also encoded by the allosteric factors $a$ and $b$. Thus, we have another set of similar mathematical relationships:

$$
\begin{aligned}
& k_{10,11}=a \times k_{4,5}, \\
& k_{9,10}=a \times k_{3,4}, \\
& k_{8,9}=a \times k_{2,3}, \\
& k_{7,8}=a \times k_{1,2}, \\
& k_{8,7}=b^{-1} \times k_{2,1}, \\
& k_{9,8}=b^{-1} \times k_{3,2}, \\
& k_{10,9}=b^{-1} \times k_{4,3}, \\
& k_{11,10}=b^{-1} \times k_{5,4} .
\end{aligned}
$$

Overall, this allosteric coupling between activation and inactivation can explain the apparently contradictory findings that inactivation appears strongly voltage-sensitive, but 
only minimal electrical charge is detected to move within the channel during inactivation (Armstrong and Bezanilla, 1977). Generally, allosteric factors, such as the $a$ and $b$ quantities above, are unknown and need to be determined from the data or estimated.

Finally, the last assumption is that the channel satisfies the condition of microscopic reversibility, i.e., no energy input is required for gating and opening. Under this condition, for any reaction loop in the model, the clockwise product of rates around the loop must equal the counterclockwise product (Song and Magleby, 1994; Rothberg and Magleby, 2001; Colquhoun, 2004). As the model in Fig. 2.2A has five independent loops, the following mathematical relationships must hold true:

$$
\begin{aligned}
& k_{1,2} \times k_{2,8} \times k_{8,7} \times k_{7,1}=k_{2,1} \times k_{8,2} \times k_{7,8} \times k_{1,7}\left(\text { for the } \mathrm{C}_{1}-\mathrm{C}_{2}-\mathrm{I}_{8}-\mathrm{I}_{7}-\mathrm{C}_{1} \text { loop}\right), \\
& k_{2,3} \times k_{3,9} \times k_{9,8} \times k_{8,2}=k_{3,2} \times k_{9,3} \times k_{8,9} \times k_{2,8}\left(\mathrm{C}_{2}-\mathrm{C}_{3}-\mathrm{I}_{9}-\mathrm{I}_{8}-\mathrm{C}_{2}\right), \\
& k_{3,4} \times k_{4,10} \times k_{10,9} \times k_{9,3}=k_{4,3} \times k_{10,4} \times k_{9,10} \times k_{3,9}\left(\mathrm{C}_{3}-\mathrm{C}_{4}-\mathrm{I}_{10}-\mathrm{I}_{9}-\mathrm{C}_{3}\right), \\
& k_{4,5} \times k_{5,11} \times k_{11,10} \times k_{10,4}=k_{5,4} \times k_{11,5} \times k_{10,11} \times k_{4,10}\left(\mathrm{C}_{4}-\mathrm{C}_{5}-\mathrm{I}_{11}-\mathrm{I}_{10}-\mathrm{C}_{4}\right), \\
& k_{5,6} \times k_{6,12} \times k_{12,11} \times k_{11,5}=k_{6,5} \times k_{12,6} \times k_{11,12} \times k_{5,11}\left(\mathrm{C}_{5}-\mathrm{O}_{6}-\mathrm{I}_{12}-\mathrm{I}_{11}-\mathrm{C}_{5}\right) .
\end{aligned}
$$

\subsubsection{Voltage-dependent and ligand-dependent rate constants}

Some of the mathematical relationships used to express parameter constraints may involve rate constants that are functions of membrane potential. Unless otherwise specified, all these mathematical relationships must be true for any membrane potential value. For example, the scaling relationship $k_{3,4}=2 \times k_{4,5}$ in Eq. 4 can be expanded as follows:

$$
k_{3,4}^{0} \times \exp \left(k_{3,4}^{1} \times V\right)=2 \times k_{4,5}^{0} \times \exp \left(k_{4,5}^{1} \times V\right)
$$


A logarithm transformation can be applied to convert products into sums:

$$
\ln \left(k_{3,4}^{0}\right)+k_{3,4}^{1} \times V=\ln (2)+\ln \left(k_{4,5}^{0}\right)+k_{4,5}^{1} \times V .
$$

Rearranging the terms gives:

$$
\ln \left(k_{3,4}^{0}\right)-\ln \left(k_{4,5}^{0}\right)+V \times\left(k_{3,4}^{1}-k_{4,5}^{1}\right)=\ln (2) .
$$

This relationship must be true when $V=0$, in which case it simplifies to:

$$
\ln \left(k_{3,4}^{0}\right)-\ln \left(k_{4,5}^{0}\right)=\ln (2)
$$

Using this result in Eq. 10, results in:

$$
k_{3,4}^{1}-k_{4,5}^{1}=0 \text {. }
$$

Thus, to enforce a scaling relationship between two voltage-dependent rate constants, the two mathematical relationships above (Eqs. 11 and 12) must be simultaneously satisfied. The same reasoning can be applied to other types of constraints. For example, after taking the logarithm and rearranging the terms, the first loop balance constraint in Eq. 7 becomes:

$$
\begin{array}{r}
(13) \quad \ln \left(k_{1,2}^{0}\right)-\ln \left(k_{2,1}^{0}\right)+\ln \left(k_{2,8}^{0}\right)-\ln \left(k_{8,2}^{0}\right)+\ln \left(k_{8,7}^{0}\right)-\ln \left(k_{7,8}^{0}\right)+ \\
\ln \left(k_{7,1}^{0}\right)-\ln \left(k_{1,7}^{0}\right)+V\left(k_{1,2}^{1}-k_{2,1}^{1}+k_{2,8}^{1}-k_{8,2}^{1}+k_{8,7}^{1}-k_{7,8}^{1}+k_{7,1}^{1}-k_{1,7}^{1}\right)=0 .
\end{array}
$$

For the above equation to be true, two mathematical relationships must be simultaneously satisfied:

$$
\ln \left(k_{1,2}^{0}\right)-\ln \left(k_{2,1}^{0}\right)+\ln \left(k_{2,8}^{0}\right)-\ln \left(k_{8,2}^{0}\right)+\ln \left(k_{8,7}^{0}\right)-\ln \left(k_{7,8}^{0}\right)+
$$

$$
\ln \left(k_{7,1}^{0}\right)-\ln \left(k_{1,7}^{0}\right)=0 \quad \text { and }
$$




$$
k_{1,2}^{1}-k_{2,1}^{1}+k_{2,8}^{1}-k_{8,2}^{1}+k_{8,7}^{1}-k_{7,8}^{1}+k_{7,1}^{1}-k_{1,7}^{1}=0 .
$$

Some kinetic mechanisms involve state transitions associated with the binding of a ligand (Grosman et al., 2000; Burzomato et al., 2004; Akk et al., 2005). For example, a relationship where one ligand-dependent rate constant $k_{\mathrm{ij}}$ is scaled by a constant factor $c$ to another ligand-dependent rate constant $k_{\mathrm{kl}}$ can be expanded as follows:

$$
\begin{aligned}
& k_{\mathrm{ij}}^{0} \times[L]=c \times k_{\mathrm{kl}}^{0} \times[L] \Rightarrow \\
& \ln \left(k_{\mathrm{ij}}^{0}\right)+\ln ([L])=\ln (c)+\ln \left(k_{\mathrm{kl}}^{0}\right)+\ln ([L]),
\end{aligned}
$$

with the final solution:

$$
\ln \left(k_{\mathrm{ij}}^{0}\right)-\ln \left(k_{\mathrm{kl}}^{0}\right)=\ln (c) .
$$

Relationships involving voltage-dependent or ligand-dependent rate constants have some special restrictions that have a simple mathematical provenance: $i$ ) if a rate is scaled to another rate, their voltage sensitivities must be equal (or trivially zero). Thus, a voltagedependent rate cannot be scaled to a non-voltage-dependent rate, except for a single voltage value; ii) if a loop involves only one voltage-dependent transition, then the forward and backward voltage sensitivity factors for that transition must be equal (or zero)(Colquhoun and Hawkes, 1995a). A more typical and useful scenario would require at least two voltage-dependent transitions in the loop; and iii) a mathematical relationship that involves ligand-dependent transitions cannot be satisfied for all ligand concentrations, unless the algebraic sum of all the $\ln ([$ Ligand $])$ terms is equal to zero. Thus, a ligand-dependent rate cannot be scaled to a non-ligand-dependent rate, except for a single concentration value. In the case of microscopic reversibility, this condition 
requires that the clockwise and counterclockwise transitions around the loop involve the same number of ligand-dependent steps. When the channel binds multiple types of ligands, each type must satisfy these conditions. Models formulated without taking these precautions are, in principle, physically unrealistic.

\subsubsection{Allosteric and other multiplicative factors}

Multiplicative factors can be introduced in the rate constant equation to express a variety of parameter constraints. One obvious application is to implement allosteric model behavior, as previously discussed, where the $a$ and $b$ factors multiply the rate constant

pre-exponential term $k_{\mathrm{ij}}^{0}$. However, multiplicative factors can also be introduced within the exponential in Eq. 1. So far, we lumped the voltage sensitivity as a single factor, $k_{\mathrm{ij}}^{1}$, but in fact we may need to consider the other quantities in Eq. 2. For example, one may want to introduce explicit temperature dependence for a rate in the case of thermosensitive proteins. In this case, $k_{\mathrm{ij}}^{1}$ can be factorized by the following constraint expression:

$$
k_{\mathrm{ij}}^{1}=a_{k} \times C_{k},
$$

where $a_{\mathrm{k}}$ is a multiplicative factor that stands for $\delta_{\mathrm{ij}} \times z_{\mathrm{ij}}$, and $C_{\mathrm{k}}$ is a numerical constant equal to $F / R T$, as in Eq. 2. This approach would make it possible to mix data collected at different temperatures, in the same way as one can already account for different voltages or ligand concentrations. 
As another example, one may want to enforce a relationship between the $\delta_{\mathrm{ij}}$ and $\delta_{\mathrm{ji}}$ values for a given transition, such as $\delta_{\mathrm{ij}}=1-\delta_{\mathrm{ji}}$. In this case, assuming that $z_{\mathrm{ij}}$ and $z_{\mathrm{ji}}$ are known quantities, the following constraint expressions will apply:

$$
k_{\mathrm{ij}}^{1}=a_{\mathrm{k} 1} \times C_{k}, \quad k_{\mathrm{ji}}^{1}=a_{\mathrm{k} 2} \times C_{k}, \quad a_{k}=1-a_{\mathrm{k} 2},
$$

where $a_{\mathrm{k} 1}$ and $a_{\mathrm{k} 2}$ are multiplicative factors that stand for $\delta_{\mathrm{ij}}$ and $\delta_{\mathrm{j} j}$, respectively, and $C_{\mathrm{k}}$ is a numerical constant equal to $\mathrm{z} F / R T$, where $z=z_{\mathrm{ij}}=z_{\mathrm{ji}}$.

Because of the logarithm transformation discussed before, multiplicative factors are subject to some restrictions. Thus, a pre-exponential parameter $k_{\mathrm{ij}}^{0}$ can only be constrained to an unlimited product of multiplicative factors and other pre-exponential parameters, each raised to an arbitrary power:

$$
k_{\mathrm{ij}}^{0}=C \times \prod_{k} a_{k}{ }^{C_{k}} \times \prod_{\mathrm{mn}} k_{\mathrm{mn}}^{0}{ }^{C_{m n}},
$$

where $C$ is a positive numerical constant. Taking the logarithm from $k_{\mathrm{ij}}^{0}$ will convert this product into a linear sum. In contrast, an exponential parameter $k_{\mathrm{ij}}^{1}$ can only be constrained to an unlimited sum of multiplicative factors and other exponential parameters, each multiplied by an arbitrary numerical constant:

$$
k_{\mathrm{ij}}^{1}=C+\sum_{k} C_{k} \times a_{k}+\sum_{\mathrm{mn}} C_{\mathrm{mn}} \times k_{\mathrm{mn}}^{1},
$$

where $C$ is an arbitrary constant. As explained further, a given multiplicative factor can only be used as a pre-exponential-type factor, as in Eq. 19, or as an exponential-type factor, as in Eq. 20, but not as both. 


\subsubsection{Inequality constraints}

So far, the considered parameter constraints have only been formulated as mathematical equalities. However, prior knowledge may also be expressed through inequality parameter constraints. First, there is a physical requirement that all pre-exponential rate parameters $k_{\mathrm{ij}}^{0}$ must be greater than zero, since transition frequencies are positive numbers. Likewise, quantities that multiply rate constants, such as the $a$ and $b$ allosteric factors above, must also be restricted to positive values in order to keep rates positive. Both of these constraints are automatically handled by the logarithm transformation of variable. In contrast, the exponential factors $k_{\mathrm{ij}}^{1}$ are in principle free to take any value in the $(-\infty,+\infty)$ range, but they can also be restricted. One could think of a variety of other inequality constraints. For example, limiting the values of voltage-sensitivity parameters $k_{\mathrm{ij}}^{1}$ to be greater than zero for voltage sensor activation rates, and less than zero for deactivation rates:

$$
\begin{aligned}
k_{\mathrm{ij}}^{1} & \geq 0, & & \text { (activation) } \\
k_{\mathrm{ji}}^{1} & \leq 0 . & & \text { (deactivation) }
\end{aligned}
$$

Applying any of these constraints could be a useful working hypothesis during the initial stages of formulating a model. Subsequently, these constraints could be relaxed. In reality, the forward and backward values can both have the same sign: as long as the activation value is more positive than the deactivation value $\left(k_{\mathrm{ij}}^{1} \geq k_{\mathrm{ji}}^{1}\right)$, the channel will be more activated at more positive membrane potentials, as is the case with Nav and other voltage-gated channels. To give another hypothetical example, one may want the 
ratio between two rate constants at a certain membrane potential $V_{0}$ to be smaller than a numerical constant $c$ :

$$
\begin{aligned}
& k_{\mathrm{ij}} / k_{\mathrm{kl}} \leq c \Rightarrow \\
& \left(k_{\mathrm{ij}}^{0} \times \exp \left(k_{\mathrm{ij}}^{1} \times V_{0}\right)\right) /\left(k_{\mathrm{kl}}^{0} \times \exp \left(k_{\mathrm{kl}}^{1} \times V_{0}\right)\right) \leq c \Rightarrow \\
& \ln \left(k_{\mathrm{ij}}^{0}\right)-\ln \left(k_{\mathrm{kl}}^{0}\right)+V_{0} \times\left(k_{\mathrm{ij}}^{1}-k_{\mathrm{kl}}^{1}\right) \leq \ln (c) .
\end{aligned}
$$

All of these " $\leq$ " or " $\geq$ " inequalities can be converted to equality relationships by subtracting or adding, respectively, a positive quantity to the right-hand side of the inequality.

For example, in Eq. 22, we can subtract $z^{2}$, a quantity that by definition is positive:

$$
\ln \left(k_{\mathrm{ij}}^{0}\right)-\ln \left(k_{\mathrm{kl}}^{0}\right)+V_{0} \times\left(k_{\mathrm{ij}}^{1}-k_{\mathrm{kl}}^{1}\right) \leq \ln (c)-z^{2} .
$$

As long as the inequality condition in Eq. 23 is satisfied for $z=0$, we can find a value for $z$ that converts the inequality into an equality:

$$
\ln \left(k_{\mathrm{ij}}^{0}\right)-\ln \left(k_{\mathrm{kl}}^{0}\right)+V_{0} \times\left(k_{\mathrm{ij}}^{1}-k_{\mathrm{kl}}^{1}\right)=\ln (c)-z^{2}
$$

If we want the above ratio between two rate constants to be smaller than a numerical constant at any voltage $V$, not just at $V_{0}$, then we have:

$$
\ln \left(k_{\mathrm{ij}}^{0}\right)-\ln \left(k_{\mathrm{kl}}^{0}\right)+V \times\left(k_{\mathrm{ij}}^{1}-k_{\mathrm{kl}}^{1}\right)=\ln (c)-z^{2} .
$$


Since this is an equality, we follow the same reasoning as for equality constraints: the above relationship must also be true when $V=0$, and thus we obtain two simultaneous relationships:

$$
\begin{aligned}
& \ln \left(k_{\mathrm{ij}}^{0}\right)-\ln \left(k_{\mathrm{kl}}^{0}\right)=\ln (c)-z^{2}, \\
& k_{\mathrm{ij}}^{1}-k_{\mathrm{kl}}^{1}=0 .
\end{aligned}
$$

In the jargon of optimization theory, $z$ is called a "slack" variable (Fletcher, 2013). With equality constraints, one has to find a set of model parameters that satisfy a set of relationships. When inequalities are added to the model and transformed into equalities using slack variables, one has to find both a set of model parameters and a set of slack variables that together satisfy the constraint relationships. A slack variable is not a true parameter of the model, but merely a variable that is temporarily used to handle inequality constraints during the search for optimum parameters. For " $\geq$ " inequalities, we must add $z^{2}$ to the right-hand side, rather than subtract it. Very importantly, the quantity added or subtracted via slack variables must take positive values, which is why we use $z^{2}$ and not $z$. The reason for converting inequality relationships to equalities is to have all linear constraints handled by the same linear algebra mathematical formalism.

\section{$\underline{\text { 2.5.5 Model parameters }}$}

As discussed in the previous section, the core parameters of a kinetic model are $k_{\mathrm{ij}}^{0}$ and $k_{\mathrm{ij}}^{1}$, together with some optional multiplicative factors $a_{k}$ that describe allosteric coupling or other properties (e.g., the $a$ and $b$ allosteric factors in the model shown in Fig. 2.2B) or help parameterizing the rate constants in more detail. However, other parameters $q_{l}$ may 
also be added to the modeling framework, depending on the application. These external parameters are not necessarily present in any of the rate constant expressions. Instead, they may describe the data or experimental variables. For example, when fitting macroscopic currents, one may also need to estimate the number of channels in the record, or the single channel conductance (Milescu, 2005). Thus, we define a set $\mathbf{K}$, of size $N_{\mathrm{K}}$, which contains all the model parameters:

$$
\boldsymbol{K}=\left\{k_{\mathrm{ij}}^{0}, k_{\mathrm{ij}}^{1}, a_{k}, q_{l}\right\}
$$

These quantities, which we term "rate constant parameters" (pre-exponential $k_{\mathrm{ij}}^{0}$ and exponential $\left.k_{\mathrm{ij}}^{1}\right)$, "multiplicative factors" $\left(a_{k}\right)$, and "external parameters" $\left(q_{l}\right)$, may be involved in the mathematical relationships that express parameter constraints, as discussed further.

\subsubsection{A general equation for linear parameter constraints}

All the mathematical relationships that were used to implement the assumptions made for the Nav model in Fig. 2.2A have something in common: regardless of type (scaling, microscopic reversibility, etc.), each of the above equality and inequality parameter constraints results in one or two equations involving $\ln \left(k_{\mathrm{ij}}^{0}\right), k_{\mathrm{ij}}^{1}, a_{k}$, and $z^{2}$, each multiplied by a constant. Although not shown in the above examples, these relationships can also contain any external parameter $q_{1}$. Thus, a general form that covers all these examples can be written as follows:

$$
\begin{aligned}
& \sum_{\mathrm{I}, \mathrm{j}}\left[C_{\mathrm{ij}}^{0} \times \ln \left(k_{\mathrm{ij}}^{0}\right)\right]+\sum_{\mathrm{I}, \mathrm{j}}\left[C_{\mathrm{ij}}^{1} \times k_{\mathrm{ij}}^{1}\right]+\sum_{k}\left[C_{k} \times f_{k}\left(a_{k}\right)\right]+\sum_{l}\left[C_{l} \times f_{l}\left(q_{l}\right)\right]=C, \\
& \text { (equality) }
\end{aligned}
$$




$$
\sum_{\mathrm{i}, \mathrm{j}}\left[C_{\mathrm{ij}}^{0} \times \ln \left(k_{\mathrm{ij}}^{0}\right)\right]+\sum_{\mathrm{i}, \mathrm{j}}\left[C_{\mathrm{ij}}^{1} \times k_{\mathrm{ij}}^{1}\right]+\sum_{k}\left[C_{k} \times f_{k}\left(a_{k}\right)\right]+\sum_{l}\left[C_{l} \times f_{l}\left(q_{l}\right)\right]=C+
$$

$C_{z} \times z^{2}, \quad$ (inequality)

where $f_{k}$ is an invertible function of the multiplicative factor $a_{k}$ (e.g., $f_{k}\left(a_{k}\right)=\ln \left(a_{k}\right)$, or $\left.f_{k}\left(a_{k}\right)=a_{k}\right)$, and $f_{l}$ is an invertible function of the external model parameter $q_{l}$. The $C_{\mathrm{ij}}^{0}, C_{i j}^{1}, C_{k}, C_{l}, C$, and $C_{z}$ quantities are numerical constants, with $C_{z}=1$ for a " $\geq$ " inequality, and $C_{z}=-1$ for a " $\leq$ " inequality.

Specific parameter constraints (e.g., scaling one rate constant to another) can be obtained from the general equation by selecting a subset of $\ln \left(k_{\mathrm{ij}}^{0}\right), k_{\mathrm{ij}}^{1}, f_{k}\left(a_{k}\right)$, and $f_{l}\left(q_{l}\right)$ via nonzero multiplication constants $C_{\mathrm{ij}}^{0}, C_{i j}^{1}, C_{k}$, or $C_{l}$. As shown in the above examples, a variety of useful constraints can be implemented using this mechanism: making a rate equal to a constant, scaling two rates by a constant factor, scaling two rates by a variable factor, constraining the total charge for a set of transitions, enforcing microscopic reversibility, constraining a reaction loop out of microscopic balance, restricting a model parameter to a range, expressing explicit temperature dependence, etc. Some of these constraints will require a single mathematical relationship, while others will require two. We note that using multiplicative factors in constraints generally makes sense when the same factor is used in multiple relationships. Otherwise, these factors can be simply calculated after the parameters are estimated.

\subsubsection{Converting between model parameters and free parameters}

One can verify that the model in Fig. 2.2B was parameterized in such a way as to implicitly satisfy most assumptions: identical and independent voltage sensors, allosteric 
coupling of inactivation to activation, and microscopic reversibility. For example, the condition of identical and independent voltage sensors is enforced by the 4, 3, 2, 1, or 1 , 2, 3, 4 factors multiplying the $\alpha_{\mathrm{m}}$ or $\beta_{\mathrm{m}}$ quantities, respectively. In other words, any values can be assigned to the model parameters, $\alpha_{m}^{0}, \alpha_{m}^{1}, a, b$, etc., and the assumptions will be automatically satisfied. There is only one exception: the $\mathrm{C}_{5}-\mathrm{O}_{6}-\mathrm{I}_{12}-\mathrm{I}_{11}-\mathrm{C}_{5}$ reaction loop is not implicitly balanced. In this case, the balance equation (i.e., $\alpha_{\mathrm{mo}} \times \beta_{\mathrm{ho}} \times \beta_{\mathrm{mh}} \times$ $\left.b^{-4} \times \alpha_{h}=\beta_{\mathrm{mo}} \times a^{4} \times \beta_{h} \times \alpha_{\mathrm{mh}} \times \alpha_{\mathrm{ho}}\right)$ is not true by definition. Instead, it must be enforced by choosing an appropriate set of numerical values for all the parameters involved. In contrast, all the other loops are automatically balanced (e.g., $4 \alpha_{m} \times \beta_{h} \times$ $\left.a \times \beta_{m} \times b^{-1} \times \alpha_{h}=\beta_{m} \times \beta_{h} \times 4 \alpha_{m} \times a \times \alpha_{h} \times b^{-1}\right)$.

In general, to formulate a parameterization that implicitly satisfies all parameter constraints, one must be able to identify a subset of independent model parameters that can be estimated by the optimization engine, and a subset of dependent parameters that can be simply derived from the independent ones. This is exactly how the model in Fig. 2.2B was formulated. However, finding this parameterization is not trivial in some cases (Colquhoun, 2004). Moreover, it is not clear to us how constraints that are defined by inequality relationships would be handled by this type of parameterization. A potentially easier and certainly more flexible strategy is to define the constraints as an invertible transformation $f_{C}$ between the set of interdependent model parameters $\mathbf{K}$ and a set of independent, or "free", parameters X:

$$
\begin{aligned}
\boldsymbol{X} & =f_{C}(\boldsymbol{K}), \\
\boldsymbol{K} & =f_{C}{ }^{-1}(\boldsymbol{X})
\end{aligned}
$$


Thus, the model is defined by the $\mathbf{K}$ parameters, which are interdependent and thus cannot take arbitrary values, but only those values that satisfy the user-defined parameter constraints. In contrast, the $\mathbf{X}$ parameters are independent of each other and are "free" to take any value in the $(-\infty,+\infty)$ range. We emphasize that the $\mathbf{X}$ parameters are not a subset of $\mathbf{K}$, as explained below. These free parameters are passed to a model-blind optimizer that can search without any constraint in the parameter space defined by $\mathbf{X}$, where it finds a solution that best explains the data. This optimal solution can be translated from the free parameter space back into the model parameter space, via the $f_{C}^{-1}$ transformation.

If we want to implement the types of parameter constraints listed in the above examples, how do we define the $f_{C}$ and $f_{C}{ }^{-1}$ transformations that translate the model parameters $\mathbf{K}$ into the free parameters $\mathbf{X}$, and vice versa? In preparation for this, we need to recognize that the left-hand side of the generalized Eq. 28 or 29 is nonlinear with respect to $k_{\mathrm{ij}}^{0}$ and $a_{k}$, and perhaps to some external parameter $q_{l}$. However, we can make the following invertible transformations of variable:

$$
\begin{aligned}
\varepsilon_{\mathrm{ij}}^{0} & =\ln \left(k_{\mathrm{ij}}^{0}\right), \\
k_{\mathrm{ij}}^{0} & =\exp \left(\varepsilon_{\mathrm{ij}}^{0}\right), \\
\varphi_{k} & =f_{k}\left(a_{k}\right), \\
a_{k} & =f_{k}^{-1}\left(\varphi_{k}\right), \\
\phi_{l} & =f_{l}\left(q_{l}\right), \\
q_{l} & =f_{l}^{-1}\left(\phi_{l}\right) .
\end{aligned}
$$


If the multiplicative factor $a_{k}$ is an allosteric factor or a similar quantity that multiplies a rate constant $k_{\mathrm{ij}}$, then $f_{k}\left(a_{k}\right)$ is $\ln \left(a_{k}\right)$, which is invertible. If $a_{k}$ is a factor that multiplies a voltage sensitivity parameter $k_{\mathrm{ij}}^{1}$, then $f_{k}\left(a_{k}\right)$ is the identity function $\varphi_{k}=a_{k}$, which is invertible as well. Similar reasoning applies to the external parameters $q_{l}$. For example, if $q_{l}$ refers to the number of channels, we can also use the logarithm transformation (Milescu, 2005). In all cases, the logarithm has two desirable effects: it restricts the variables to positive values, and it scales the parameters to more similar values relative to each other, helping the optimization engine to find the solution.

We can rewrite the generalized Eqs. 28 and 29 with these transformations of variable:

$$
\begin{aligned}
& \sum_{\mathrm{i}, \mathrm{j}}\left[C_{\mathrm{ij}}^{0} \times \varepsilon_{\mathrm{ij}}^{0}\right]+\sum_{\mathrm{i}, \mathrm{j}}\left[C_{\mathrm{ij}}^{1} \times k_{\mathrm{ij}}^{1}\right]+\sum_{k}\left[C_{k} \times \varphi_{k}\right]+\sum_{l}\left[C_{l} \times \phi_{l}\right]=C, \quad \text { (equality) } \\
& \sum_{\mathrm{i}, \mathrm{j}}\left[C_{\mathrm{ij}}^{0} \times \varepsilon_{\mathrm{ij}}^{0}\right]+\sum_{\mathrm{i}, \mathrm{j}}\left[C_{\mathrm{ij}}^{1} \times k_{\mathrm{ij}}^{1}\right]+\sum_{k}\left[C_{k} \times \varphi_{k}\right]+\sum_{l}\left[C_{l} \times \phi_{l}\right]=C+C_{z} \times z^{2}
\end{aligned}
$$
(inequality)

The left-hand side of these equations is now linear with respect to $\varepsilon_{\mathrm{ij}}^{0}, k_{\mathrm{ij}}^{1}, \varphi_{k}$, and $\phi_{k}$. Next, we define a vector $\mathbf{R}$, of dimension $N_{\mathrm{R}}$, with elements that correspond to $\varepsilon_{\mathrm{ij}}^{0}, k_{\mathrm{ij}}^{1}$, $\varphi_{k}$, and $\phi_{k}$. For a more intuitive notation, we refer to an element of $\mathbf{R}$ as $r_{\mathrm{i}}$, when its type is unspecified, or as $r_{\mathrm{ij}}^{0}, r_{\mathrm{ij}}^{1}, r_{k}$, or $r_{k}$, when we emphasize its identity as a specific type of model parameter $\left(k_{\mathrm{ij}}^{0}, k_{\mathrm{ij}}^{1}, a_{k}\right.$, or $q_{k}$, respectively). $\mathbf{R}$ has the same size as $\mathbf{K}\left(N_{\mathrm{R}}=N_{\mathrm{K}}\right)$. Thus, a parameter constraint is expressed as a linear relationship between the elements $r_{i}$ of $\mathbf{R}$, as follows:

$$
\begin{array}{ll}
\sum_{i} C_{i} \times r_{i} & =C, \\
\sum_{i} C_{i} \times r_{i}=C+C_{z} \times z^{2}, & \text { (inequality) }
\end{array}
$$


where $C_{i}$ stands for one of the numerical constants $C_{\mathrm{ij}}^{0}, C_{\mathrm{ij}}^{1}, C_{k}$, or $C_{l}$, respectively. Then, assuming that we have $N_{\mathrm{C}}$ constraint relationships, we can write the generalized constraint Eq. 34 or 35 above in a more compact matrix form (Qin, 1996; Fletcher, 2013), as follows:

$$
M \times R=V
$$

where $\mathbf{M}$ is a matrix of dimension $N_{\mathrm{C}} \mathrm{x} N_{\mathrm{R}}$ and $\mathbf{V}$ is a vector of dimension $N_{\mathrm{C}}$. Each row of $\mathbf{M}$ corresponds to the numerical constants on the left-hand side of the generalized Eq. 34 or 35, while each element of $\mathbf{V}$ represents the right-hand side of Eq. 34 or 35 :

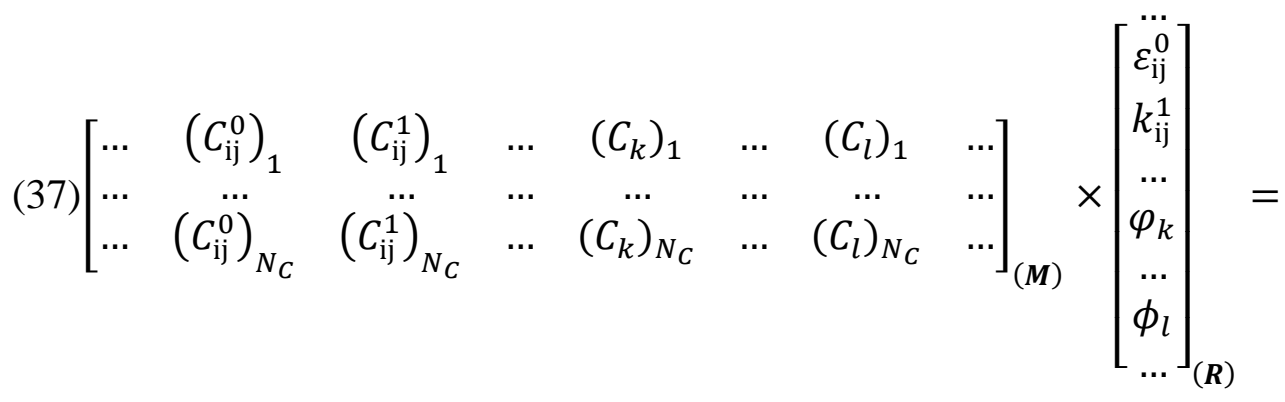
$\left[\begin{array}{cc}(C)_{1} & \text { or }\left(C+C_{z} \times z^{2}\right)_{1} \\ (C)_{N_{C}} & \text { or }\left(C+C_{z} \times z^{2}\right)_{N_{C}}\end{array}\right]_{(V)}$.

Thus, each linear relationship between the $\varepsilon_{\mathrm{ij}}^{0}, k_{\mathrm{ij}}^{1}, \varphi_{k}$, and $\phi_{l}$ variables is encoded by row $c$ of matrix $\mathbf{M}$ and element $c$ of vector $\mathbf{V}$. Equation 36 encapsulates in matrix form all the linear parameter constraints imposed on the model, including both equality and inequality relationships.

Although they linearize the constraint relationships, the transformed model parameters $\mathbf{R}$ are still interdependent through the constraint relationships. How do we remove the 
interdependence and convert $\mathbf{R}$ into the set of free parameters $\mathbf{X}$ ? Ignoring for now that $\mathbf{V}$ is not a constant vector, because it depends nonlinearly on the optional slack variables $z$, we can take advantage of the linear form of the matrix equation $\boldsymbol{M} \times \boldsymbol{R}=\boldsymbol{V}$ and express $\mathbf{R}$ as a linear function of $\mathbf{X}$ (Qin, 1996), and vice versa:

$$
\begin{aligned}
& R=A \times X+B \\
& X=A^{-1} \times R
\end{aligned}
$$

where the vector $\mathbf{X}$, of dimension $N_{\mathrm{X}}=N_{\mathrm{R}}-N_{\mathrm{C}}$, contains the independent parameters. Note that Eq. 39 is obtained from $\boldsymbol{X}=\boldsymbol{A}^{-1} \times(\boldsymbol{R}-\boldsymbol{B})$, because $\mathbf{A}^{-1}$ multiplied by $\mathbf{B}$ is equal to a zero vector. The matrix $\mathbf{A}$, of dimension $N_{\mathrm{R}} \times N_{\mathrm{X}}$, and the vector $\mathbf{B}$, of dimension $N_{\mathrm{R}}$, can be determined from $\mathbf{M}$ and $\mathbf{V}$ using the singular value decomposition (Golub and Reinsch, 1970). First, $\mathbf{M}$ is decomposed as follows:

$$
\boldsymbol{M}=\boldsymbol{U}_{M} \times \boldsymbol{S}_{M} \times \boldsymbol{V}_{M}{ }^{T}
$$

where $\mathbf{U}_{\mathrm{M}}$ is an orthogonal matrix of dimension $N_{\mathrm{C}} \mathrm{x} N_{\mathrm{C}}, \mathbf{V}_{\mathrm{M}}$ is an orthogonal matrix of dimension $N_{\mathrm{R}} \mathrm{x} N_{\mathrm{R}}$, and $\mathbf{S}_{\mathrm{M}}$ is a diagonal matrix of dimension $N_{\mathrm{C}} \mathrm{x} N_{\mathrm{R}}$ that contains the singular values of matrix $\mathbf{M}$. Then, $\mathbf{A}$ can be extracted as a submatrix of $\mathbf{V}_{\mathbf{M}}$ :

$$
\boldsymbol{A}_{i=1 \ldots N_{R}, \mathrm{j}=1 \ldots N_{X}}=\boldsymbol{V}_{\mathrm{M}_{i=1 \ldots N_{R}, j=N_{C} \ldots N_{R}}}
$$

The inverse of the $\mathbf{A}$ matrix, $\mathbf{A}^{-1}$, is similarly obtained from $\mathbf{V}_{\mathbf{M}}{ }^{-1}$. Since $\mathbf{V}_{\mathbf{M}}$ is orthogonal, $\mathbf{V}_{M}{ }^{-1}$ is simply equal to $\mathbf{V}_{M}$ transposed $\left(\mathbf{V}_{M}{ }^{T}\right)$. Then, $\mathbf{B}$ can be calculated as follows:

$$
B=M^{+} \times V
$$


where the matrix $\boldsymbol{M}^{+}$, of dimension $N_{\mathrm{R}}$ x $N_{\mathrm{C}}$, is the pseudo-inverse of $\mathbf{M}$ and can be calculated as follows:

$$
\boldsymbol{M}^{+}=\boldsymbol{V}_{M} \times \boldsymbol{S}_{M}^{+} \times \boldsymbol{U}_{M}{ }^{T}
$$

where $\mathbf{S}_{\mathrm{M}}{ }^{+}$is obtained from $\mathbf{S}_{\mathrm{M}}$ by replacing all non-zero diagonal elements (the singular values) with their inverse. With the $\mathbf{A}$ and $\mathbf{B}$ matrices obtained as above, we can now calculate $\mathbf{R}$ from $\mathbf{K}$, and then $\mathbf{X}$ from $\mathbf{R}$, and vice versa.

How do we deal with inequality constraints and slack variables? We found a simple solution, though perhaps not immediately obvious. First, we define a vector $\mathbf{Z}$, of dimension $N_{\mathrm{Z}}$, where $N_{\mathrm{Z}}$ is equal to the number of inequality constraints. This vector contains all the slack variables, one for each inequality constraint. Then, we define another vector $\overline{\boldsymbol{X}}$, which is the union of $\mathbf{X}$ and $\mathbf{Z}$ :

$$
\bar{X}=\boldsymbol{X} \cup \boldsymbol{Z}
$$

The size of $\overline{\boldsymbol{X}}$ is equal to $N_{\mathrm{X}}+N_{\mathrm{Z}}$, The slack variables $\mathbf{Z}$ are arbitrary and thus independent of each other and of the free parameters $\mathbf{X}$. Hence, the elements of $\overline{\boldsymbol{X}}$ are also independent of each other, and represent the free parameters given to the optimizer. Each time the optimizer tries a new set of free parameters $\overline{\boldsymbol{X}}$, the corresponding $\mathbf{Z}$ is used to recalculate $\mathbf{V}$ (Eq. 37), which in turn is used to recalculate $\mathbf{B}$ (Eq. 42). The A matrix remains the same, because the coefficient matrix $\mathbf{M}$ contains only constants. Thus, we can calculate the transformed model parameters $\mathbf{R}$ as follows:

$$
\begin{aligned}
& \boldsymbol{B}_{Z}=\boldsymbol{M}^{+} \times \boldsymbol{V}_{Z}, \\
& \boldsymbol{R}=\boldsymbol{A} \times \boldsymbol{X}+\boldsymbol{B}_{Z},
\end{aligned}
$$


where $\boldsymbol{B}_{Z}$ and $\boldsymbol{V}_{Z}$ are the $\mathbf{B}$ and $\mathbf{V}$ quantities calculated for a given vector $\mathbf{Z}$.

During optimization, the slack variables in $\mathbf{Z}$ are provided by the optimizer, together with $\mathbf{X}$. However, $\mathbf{Z}$ must be initialized at the beginning of the optimization from a given set of transformed model parameters $\mathbf{R}$ and the appropriate relationships in Eq. 37. Let $z_{\mathrm{c}}$ be the slack variable introduced by the inequality relationship defined by row $c$ of the constraint matrix $\mathbf{M}$. Then, $z_{\mathrm{c}}$ can be calculated with the following equation:

$$
\boldsymbol{M}_{c} \times \boldsymbol{R}=C_{c}+C_{\mathrm{zc}} \times z_{c}{ }^{2},
$$

where $\mathbf{M}_{\mathrm{c}}$ is a vector corresponding to row $c$ of $\mathbf{M}$. This equation has the obvious solution:

$$
z_{c}=\sqrt{\left(\frac{\boldsymbol{M}_{c} \times \boldsymbol{R}-C_{c}}{C_{\mathrm{zc}}}\right)} .
$$

The two-way conversion between the model parameters $\mathbf{K}$ and the free parameters $\overline{\boldsymbol{X}}$ is summarized in the flowchart in Fig. 2.3.

\subsubsection{Redundant constraints}

One should take care to prevent redundancy and use only the minimum number of mathematical relationships that are necessary to implement the assumptions of the model. Intuitively, a constraint relationship is redundant if its intended consequence is already enforced by other relationships. With our example model, one could use either the scaling $k_{7,8}=a \times k_{1,2}$, or the scaling $k_{7,8}=4 \times a \times k_{4,5}$, but not both, because it would create an additional relationship between $k_{1,2}$ and $k_{4,5}$. Similarly, the condition that the algebraic sum of voltage sensitivities around a reaction loop is equal to zero may be 
already enforced by some rate scaling relationships, and should not be duplicated. When in doubt, one could check the rank of the $\mathbf{M}$ matrix, which will be reduced by redundant constraints.

Redundant constraints may also arise from inequalities. Although tempting, using inequality relationships to enforce a range constraint on a model parameter is not possible, unfortunately, because it would result in two equality relationships that are redundant. However, this limitation can be overcome through the same mechanism that handles behavioral constraints (Navarro et al., 2018). Furthermore, although inequality constraints add slack variables to the overall set of free parameters, the total number of equality and inequality constraints must still be strictly smaller than the number of model parameters $\mathbf{K}$.

\subsubsection{Calculating the cost function and its gradients}

In a typical scenario, the cost function $F$ is an explicit function of the rate constants $k_{\mathrm{ij}}$ and of some external model parameters $q_{l}$ :

(49) $F=f\left(k_{\mathrm{ij}}, q_{l}\right)$.

Typically, $F$ would not depend explicitly on the multiplicative factors $a_{k}$, which are generally used to establish relationships between other parameters. Since the model parameters $\mathbf{K}$ can be obtained from the free parameters $\overline{\boldsymbol{X}}, F$ can also be written as a function of the free parameters $\bar{X}$ :

(50) $F=f\left(\bar{x}_{k}\right)$. 
Thus, the optimization algorithm is model-blind. In other words, even though it eventually generates a set of optimum model parameters $\boldsymbol{K}^{*}$, it actually searches for a solution in the space defined by the free parameters $\overline{\boldsymbol{X}}$. As it searches for the solution, the optimizer requires the cost function $F$ to be calculated for each proposed $\overline{\boldsymbol{X}}$. The specific expression of the cost function $F$ depends on the type of application: it could be a sum of square errors, a likelihood, or a Bayesian posterior probability, or it could be a mixture of these, when multiple types of data are bundled together (e.g., single channel and whole cell traces).

The optimizer may also require the gradients of $F$ with respect to the free parameters $\overline{\boldsymbol{X}}$, as is the case with gradient descent optimization methods (Fletcher, 2013). These gradients can be calculated by numerical approximation, but analytical calculation is more accurate and may actually be faster in some instances. To calculate the gradient of $F$ with respect to a free parameter $\bar{x}_{k}$, we have to consider that the rate constants $k_{\mathrm{ij}}$ are functions of $k_{\mathrm{ij}}^{0}$ and $k_{\mathrm{ij}}^{1}$. In turn, $k_{\mathrm{ij}}^{0}$ is a function of $\varepsilon_{\mathrm{ij}}^{0}$. We also have to consider that $q_{l}$ is a function of $\phi_{l}$. These $\varepsilon_{\mathrm{ij}}^{0}, k_{\mathrm{ij}}^{1}$, and $\phi_{l}$ quantities are entries in the $\mathbf{R}$ vector and thus are explicit functions of a free parameter $\bar{x}_{k}$. To calculate a gradient, we apply the chain differentiation rule, as follows:

$$
\frac{\partial F}{\partial \bar{x}_{k}}=\sum_{\mathrm{ij}}\left[\frac{\partial F}{\partial k_{\mathrm{ij}}} \times\left(\frac{\partial k_{\mathrm{ij}}}{\partial k_{\mathrm{ij}}^{0}} \times \frac{\partial k_{\mathrm{ij}}^{0}}{\partial r_{m}} \times \frac{\partial r_{m}}{\partial \bar{x}_{k}}+\frac{\partial k_{\mathrm{ij}}}{\partial k_{\mathrm{ij}}^{1}} \times \frac{\partial k_{\mathrm{ij}}^{1}}{\partial r_{n}} \times \frac{\partial r_{n}}{\partial \bar{x}_{k}}\right)\right]+\sum_{l}\left[\frac{\partial F}{\partial q_{l}} \times \frac{\partial q_{l}}{\partial r_{p}} \times \frac{\partial r_{p}}{\partial \bar{x}_{k}}\right]
$$

In the above equation, $r_{m}, r_{n}$, and $r_{p}$ are the elements of the $\mathbf{R}$ vector that correspond to $\varepsilon_{\mathrm{ij}}^{0}, k_{\mathrm{ij}}^{1}$, and $\phi_{l}$, respectively. The $\frac{\partial F}{\partial k_{\mathrm{ij}}}$ and $\frac{\partial F}{\partial q_{l}}$ quantities depend on the specific application, e.g., the maximum interval likelihood (Qin, 1996) or the maximum point likelihood of 
single channel data (Qin, 2000a), or the maximum likelihood of macroscopic currents (Milescu, 2005). The other partial derivatives can be calculated as follows:

$$
\frac{\partial k_{\mathrm{ij}}}{\partial k_{\mathrm{ij}}^{0}}=\frac{k_{\mathrm{ij}}}{k_{\mathrm{ij}}^{0}}, \quad \frac{\partial k_{\mathrm{ij}}^{0}}{\partial r_{m}}=k_{\mathrm{ij}}^{0}, \quad \frac{\partial k_{\mathrm{ij}}}{\partial k_{\mathrm{ij}}^{1}}=k_{\mathrm{ij}} \times V, \quad \frac{\partial k_{\mathrm{ij}}^{1}}{\partial r_{n}}=1 .
$$

The $\frac{\partial q_{l}}{\partial r_{p}}$ partial derivative depends on the specific transformation between $q_{l}$ and $\phi_{l}$, as illustrated here for the logarithm and identity transformations:

$$
\begin{array}{ll}
\frac{\partial q_{l}}{\partial r_{p}}=q_{l} & \text { if } \phi_{l}=\ln q_{l}, \\
\frac{\partial q_{l}}{\partial r_{p}}=1 & \text { if } \phi_{l}=q_{l} .
\end{array}
$$

Finally, the partial derivative of any $r_{i}$ with respect to any $\bar{x}_{k}$ takes the following form:

$$
\begin{aligned}
& \frac{\partial r_{i}}{\partial \bar{x}_{k}}=a_{\mathrm{i}, \mathrm{k}} \quad \text { if } \overline{\boldsymbol{x}}_{\boldsymbol{k}} \in \boldsymbol{X}, \\
& \frac{\partial r_{i}}{\partial \bar{x}_{k}}=2 \times m^{+}{ }_{\mathrm{i}, \mathrm{c}} \times \bar{x}_{k} \text { if } \overline{\boldsymbol{x}}_{\boldsymbol{k}} \in \boldsymbol{Z} \text { and inequality constraint is " } \geq ", \\
& \frac{\partial r_{i}}{\partial \bar{x}_{k}}=-2 \times m^{+}{ }_{\mathrm{i}, \mathrm{c}} \times \bar{x}_{k} \quad \text { if } \overline{\boldsymbol{x}}_{\boldsymbol{k}} \in \boldsymbol{Z} \text { and inequality constraint is " } \leq " .
\end{aligned}
$$

where $a_{\mathrm{i}, \mathrm{k}}$ and $m_{\mathrm{i}, \mathrm{c}}^{+}$are elements in the $\mathbf{A}$ and $\mathbf{M}^{+}$matrices, respectively. In the last two equations, the $c$ subscript is the index of the inequality constraint relationship that uses $\overline{\boldsymbol{x}}_{\boldsymbol{k}}$ as the slack variable (the index in $\mathbf{V}$, Eq. 45).

Using all these quantities, the overall analytical derivative of $F$, the cost function with respect to $\overline{\boldsymbol{x}}_{\boldsymbol{k}}$ becomes:

$$
\begin{aligned}
& \frac{\partial F}{\partial \dot{x}_{k}}=\sum_{\mathrm{ij}}\left[\frac{\partial F}{\partial k_{\mathrm{ij}}} \times k_{\mathrm{ij}} \times\left(a_{\mathrm{m}, \mathrm{k}}+V \times a_{\mathrm{n}, \mathrm{k}}\right)\right]+\sum_{l}\left[\frac{\partial F}{\partial q_{l}} \times q_{l} \times a_{\mathrm{p}, \mathrm{k}}\right] \quad \text { if } \overline{\boldsymbol{x}}_{\boldsymbol{k}} \in \boldsymbol{X}, \\
& \frac{\partial F}{\partial \bar{x}_{k}}=2 \times \bar{x}_{k} \times\left\{\sum_{\mathrm{ij}}\left[\frac{\partial F}{\partial k_{\mathrm{ij}}} \times k_{\mathrm{ij}} \times\left(m_{\mathrm{m}, \mathrm{c}}^{+}+V \times{m^{+}}_{\mathrm{n}, \mathrm{c}}\right)\right]+\sum_{l}\left[\frac{\partial F}{\partial q_{l}} \times q_{l} \times m^{+}{ }_{\mathrm{p}, \mathrm{c}}\right]\right\}
\end{aligned}
$$




$$
\begin{gathered}
\text { if } \overline{\boldsymbol{x}}_{\boldsymbol{k}} \in \boldsymbol{Z}, " \geq " \\
\frac{\partial F}{\partial \bar{x}_{k}}=-2 \times \bar{x}_{k} \times\left\{\sum_{\mathrm{ij}}\left[\frac{\partial F}{\partial k_{\mathrm{ij}}} \times k_{\mathrm{ij}} \times\left(m_{\mathrm{m}, \mathrm{c}}^{+}+V \times m^{+}{ }_{\mathrm{n}, \mathrm{c}}\right)\right]+\sum_{l}\left[\frac{\partial F}{\partial q_{l}} \times q_{l} \times m^{+}{ }_{\mathrm{p}, \mathrm{c}}\right]\right\} \\
\text { if } \overline{\boldsymbol{x}}_{\boldsymbol{k}} \in \boldsymbol{Z}, " \leq " .
\end{gathered}
$$

The above equations are given for the case of $\phi_{l}=\ln q_{l}$. The subscripts $m, n$, and $p$ used for the $a$ and $m^{+}$quantities are equal to the indices in the $\mathbf{R}$ vector that correspond to $\varepsilon_{\mathrm{ij}}^{0}$, $k_{\mathrm{ij}}^{1}$, and $\phi_{l}$, respectively.

\subsubsection{Calculating the error of the estimates}

When estimating the parameters of a model, it is important to have a measure of confidence in those estimates. The variance of a free parameter estimate measures the curvature of the cost function with respect to that parameter. Intuitively, the variance tells us how much the calculated prediction of the model will change when the value of a free parameter $\bar{x}_{k}$ is changed by a small amount. We emphasize that this change must be interpreted in the context of the specific data used in the analysis. Thus, parameters estimated with large variance are generally poorly determined, because of insufficient data, while small variance denotes a well-defined parameter.

One could calculate the variance of a free parameter estimate, $\operatorname{Var}\left(\bar{x}_{k}\right)$, from the second order partial derivative of the cost function, or could use the variance provided by some optimization engines, as is the case with the Davidon-Fletcher-Powell optimizer (Fletcher, 2013). However, when using the parameter constraints described here, the free parameters $\overline{\boldsymbol{X}}$ must be converted back to model parameters $\mathbf{K}$, and some transformation 
must be applied to the variance. Thus, the variance of a model parameter, $\operatorname{Var}\left(k_{i}\right)$, can be calculated using the following approximation (Qin, 2000a; Milescu, 2005):

$$
\operatorname{Var}\left(k_{i}\right)=\sum_{p}\left[\operatorname{Var}\left(\bar{x}_{p}\right) \times\left(\frac{\partial k_{i}}{\partial \bar{x}_{p}}\right)^{2}\right]
$$

where $\bar{x}_{p}$ is a free parameter in $\overline{\boldsymbol{X}}$. To calculate the variance for each type of model parameter $\left(k_{\mathrm{ij}}^{0}, k_{\mathrm{ij}}^{1}, a_{k}\right.$, and $\left.q_{l}\right)$, we use the chain differentiation rule, as shown above for calculating the cost function gradients. For rate constant parameters $\left(k_{\mathrm{ij}}^{0}\right.$ and $\left.k_{\mathrm{ij}}^{1}\right)$, we obtain the following:

$$
\begin{aligned}
& \operatorname{Var}\left(k_{\mathrm{ij}}^{0}\right)=\sum_{p}\left[\operatorname{Var}\left(\bar{x}_{p}\right) \times\left(k_{\mathrm{ij}}^{0} \times \frac{\partial r_{\mathrm{ij}}^{0}}{\partial \bar{x}_{p}}\right)^{2}\right], \\
& \operatorname{Var}\left(k_{\mathrm{ij}}^{1}\right)=\sum_{p}\left[\operatorname{Var}\left(\bar{x}_{p}\right) \times\left(\frac{\partial r_{\mathrm{ij}}^{1}}{\partial \bar{x}_{p}}\right)^{2}\right] .
\end{aligned}
$$

For pre-exponential and exponential multiplicative factors $a_{\mathrm{k}}$ we have the expressions:

$$
\begin{array}{ll}
\operatorname{Var}\left(a_{k}\right)=\sum_{p}\left[\operatorname{Var}\left(\bar{x}_{p}\right) \times\left(a_{k} \times \frac{\partial r_{k}}{\partial \bar{x}_{p}}\right)^{2}\right] & \text { if } a_{\mathrm{k}} \text { is pre-exponential, } \\
\operatorname{Var}\left(a_{k}\right)=\sum_{p}\left[\operatorname{Var}\left(\bar{x}_{p}\right) \times\left(\frac{\partial r_{k}}{\partial \bar{x}_{p}}\right)^{2}\right] & \text { if } a_{\mathrm{k}} \text { is exponential. }
\end{array}
$$

Finally, for external parameters $q_{1}$, the expression depends on the transformation function. For the logarithm and the identity function, we have the following expressions:

$$
\begin{array}{ll}
\operatorname{Var}\left(q_{l}\right)=\sum_{p}\left[\operatorname{Var}\left(\bar{x}_{p}\right) \times\left(q_{l} \times \frac{\partial r_{l}}{\partial \bar{x}_{p}}\right)^{2}\right] & \text { for logarithm transformation, } \\
\operatorname{Var}\left(q_{l}\right)=\sum_{p}\left[\operatorname{Var}\left(\bar{x}_{p}\right) \times\left(\frac{\partial r_{l}}{\partial \bar{x}_{p}}\right)^{2}\right] & \text { for identity transformation. }
\end{array}
$$


In Eqs. 56 through 59, the partial derivative of $r\left(r_{\mathrm{ij}}^{0}, r_{\mathrm{ij}}^{1}, r_{k}\right.$, and $\left.r_{l}\right)$ with respect to $\bar{x}_{p}$ is calculated as in Eqs. 54, depending on whether $\bar{x}_{p}$ is an element of $\mathrm{X}$ or a slack variable in $\mathrm{Z}$.

\subsection{Behavioral constraints and arbitrary parameter relationships}

A good amount of prior knowledge about the channel can be expressed as linear relationships between model parameters, resulting in constraints that can be handled with relatively straightforward linear algebra methods. However, some channel behaviors and properties cannot be easily formulated as explicit functions of model parameters, or they need nonlinear functions that are not so easily tractable. For voltage-gated channels, examples of important functional behavior include the open probability $\left(P_{\mathrm{O}}\right)$, the voltagedependence of activation or inactivation, or the use-dependent availability. These properties cannot be easily formulated as functions of rate constants, except for very simple kinetic mechanisms. Furthermore, they must be prescribed in the context of a specific stimulation protocol (e.g., voltage-clamp step protocols, etc.).

\subsubsection{Expanding the cost function}

Without explicit parameter relationships, we cannot solve behavioral constraints simply by converting model parameters into free parameters, as we did for linear parameter constraints. Likewise, we cannot use that formalism to solve any parameter constraint that cannot be written as a linear relationship between the transformed model parameters, as captured by the generalized linear constraint equations 32 and 33. Instead, the solution proposed here for handling behavioral constraints and arbitrary parameter relationships is 
to include them into the cost function. Thus, the cost function $F$, which is minimized by the optimizer in search for an optimal solution, can be expanded to include multiple components, one for each piece of experimental data and one for each constraint:

$$
\text { (60) } \quad F=\sum_{i}\left(\alpha_{i}^{Y} \times F_{i}^{Y}\right)+\sum_{j}\left(\alpha_{j}^{C} \times F_{j}^{C}\right)
$$

where $F_{i}^{Y}$ represents the cost of data component $i$ and $F_{j}^{C}$ represents the cost of behavioral constraint $j$. The $\alpha_{i j}$ quantities are relative weighting factors that multiply the cost function components. Including multiple components in the cost function is known in the optimization literature as multi-objective fitting (Druckmann et al., 2007;

Bandyopadhyay and Saha, 2013; Fletcher, 2013). For example, $F_{i}^{Y}$ could stand for newly acquired voltage-clamp data (e.g., the time course of activation and inactivation at different membrane potentials), while $F_{j}^{C}$ could be data from the literature (e.g., steady state activation and inactivation curves), or a hypothetical property recorded in a different paradigm (e.g., the open probability $P_{\mathrm{O}}$ ). The cost function components that denote constraints should be formulated in such a way that they take a value of zero when the underlying constraint is satisfied, and a very large value - relative to the data cost components - when the constraint fails, as explained further.

2.6.2 Formulating behavioral constraints and arbitrary parameter $\underline{\text { relationships }}$

Some behavioral constraints can be formulated as mathematical relationships involving simple properties of the channel. For example, we could constrain the maximum open 
probability reached during a depolarization step to take certain values or to fall within a range:

$$
P_{O}=0.5, \text { or } \quad P_{O} \leq 0.4 \text {, or } \quad 0.3 \leq P_{O} \leq 0.7
$$

An example of a parameter constraint that cannot be processed with the formalism developed in part one is restricting a rate constant pre-exponential factor $k_{\mathrm{ij}}^{0}$ to a range of values:

$$
1000 \leq k_{\mathrm{ij}}^{0} \leq 10000
$$

This range constraint cannot be handled as two linear inequality relationships, because they would be mathematically redundant, where both cannot be simultaneously satisfied. Another example is parameterizing an exponential factor $k_{\mathrm{ij}}^{1}$ as a product of more than one variable:

$$
k_{\mathrm{ij}}^{1}=C \times a \times b,
$$

where $a$ and $b$ could stand for the $\delta_{\mathrm{ij}}$ and $z_{\mathrm{ij}}$ parameters in Eq. 2, and $C$ is a constant equal to $F /(R \times T)$. Unfortunately, this equation is nonlinear.

Algebraically, any equality or inequality relationship can be converted to " $=0 "$ or to " $\geq 0$ ", respectively. Thus, the above constraints could be rewritten as follows:

$$
\begin{aligned}
& P_{O}-0.5=0, \\
& 0.4-P_{O} \geq 0, \\
& \left\{\begin{array}{l}
P_{O}-0.3 \geq 0 \\
0.7-P_{O} \geq 0
\end{array}\right\} .
\end{aligned}
$$




$$
\begin{aligned}
& \left\{\begin{array}{c}
k_{\mathrm{ij}}^{0}-1000 \geq 0 \\
10000-k_{\mathrm{ij}}^{0} \geq 0
\end{array}\right\} . \\
& k_{\mathrm{ij}}^{1}-C \times a \times b=0 .
\end{aligned}
$$

The cost function components $F^{C}$ that correspond to the above equality and inequality relationships can be formulated as follows:

$$
\begin{aligned}
& F^{C}=\alpha \times\left(P_{O}-0.5\right)^{2}, \\
& F^{C}=\alpha \times\left(0.4-P_{O}\right)^{2}, \\
& F^{C}=\alpha \times\left[\left(P_{O}-0.3\right)^{2}+\left(0.7-P_{O}\right)^{2}\right], \\
& F^{C}=\alpha \times\left[\left(k_{\mathrm{ij}}^{0}-1000\right)^{2}+\left(10000-k_{\mathrm{ij}}^{0}\right)^{2}\right], \text { and } \\
& F^{C}=\alpha \times\left(k_{\mathrm{ij}}^{1}-C \times a \times b\right)^{2},
\end{aligned}
$$

where $\alpha$ is a weighting factor with the following properties:

$$
\alpha>0
$$

for equality constraints, and

(67) $\left\{\begin{array}{l}\alpha=0 \text { if constraint } \geq 0 \\ \alpha>0 \text { if constraint }<0\end{array}\right\}$,

for inequality constraints,

where "constraint" refers to the left-hand side term of a constraint equation (Eqs. 37).

Thus, these cost function components are equal to zero when the underlying constraints are exactly satisfied, but take a positive and quadratically increasing value when the constraint relationships are not satisfied.

\subsubsection{Non-parametric behavioral constraints}


In principle, the same logic can be applied to any other model property. However, some model behaviors cannot be reduced to a single value or cannot be easily calculated theoretically. For example, many functional aspects, such as the recovery from inactivation or the use-dependence, can be empirically fitted by one or two exponentials. Unfortunately, these apparent time constants cannot be directly and easily calculated from the model, which actually predicts a larger number of exponentials, equal to the state count minus one (Milescu, 2005; Salari et al., 2016). Likewise, the voltagedependent activation curve can be well approximated and fitted by a Boltzmann equation with only two parameters, but calculating the half-activation and the sensitivity values directly from the model is generally not practical.

In cases like these, it is simpler to simulate the response of the channel to the same stimulation protocol as was used to obtain the experimental - or hypothesized - data. Then, a cost function component can be calculated as the sum of square differences between the simulated and the experimental data:

$$
F^{C}=\alpha \times \frac{1}{N} \sum_{i}\left(y_{i}-x_{i}\right)^{2},
$$

where $y_{\mathrm{i}}$ and $x_{\mathrm{i}}$ are experimental and simulated data points, respectively, and $N$ is the number of data points. In the above equation, one could use the raw data directly, point by point, or one could extract some properties from the raw data and use the points on that property curve. For example, when the stimulation protocol is designed to extract the time course of a macroscopic current, one would fit the raw data directly. In contrast, when the stimulation protocol is designed to extract a behavior, such as the recovery from inactivation, one would fit the property curve. Although extracting a property curve 
involves additional computation, it has the substantial benefit of concentrating the information on a very specific aspect of channel behavior. For example, in a curve that represents the recovery from inactivation, every data point informs directly on the apparent time constants of inactivation. Likewise, every data point in a voltage-dependent activation curve informs directly on the two parameters of the Boltzmann equation.

Whether the cost function for these non-parametric behavioral constraints is calculated from raw data or from property curves, or is based on hypothetical values, one must consider the presence of random noise and other artifacts that contaminate the experimental data. Thus, even a perfect model would not generate zero cost for the constraints, which may confuse the optimization engine. A simple solution is to reformulate the problem as an inequality:

(69) $\frac{1}{N} \sum_{i}\left(y_{i}-x_{i}\right)^{2} \leq \varepsilon$

where $\varepsilon$ is a positive constant proportional to the noise content of the data. Then, the cost function component can be written as follows:

$$
\text { (70) } F^{C}=\alpha \times\left[\varepsilon-\frac{1}{N} \sum_{i}\left(y_{i}-x_{i}\right)^{2}\right]^{2} \text {, }
$$

where $\alpha$ is a weighting factor with the following property:

$$
\left\{\begin{array}{l}
\alpha=0 \text { if } \frac{1}{N} \sum_{i}\left(y_{i}-x_{i}\right)^{2} \leq \varepsilon \\
\alpha>0 \text { if } \frac{1}{N} \sum_{i}\left(y_{i}-x_{i}\right)^{2}>\varepsilon
\end{array}\right\}
$$

Thus, if the sum of square errors between the simulated and the experimental data is less than $\varepsilon$, then the underlying constraint is considered to be satisfied. In other words, the 
model only needs to explain the constraining data components "well enough", as warranted by the inevitable noise and artifacts.

\subsubsection{A computational framework for solving behavioral constraints and}

\section{arbitrary parameter relationships}

We presented above some examples of behavioral constraints and arbitrary parameter relationships. In general, the problem we must solve is to find a model that best explains the experimental data, but also satisfies a set of equality or inequality constraints. Mathematically, the problem can be formulated as the minimization of a function subject to a set of nonlinear constraints:

$$
\begin{aligned}
& \operatorname{minimize} F(\overline{\boldsymbol{X}}) \\
& \text { such that: } h_{i}(\overline{\boldsymbol{X}})=0, i=1 \ldots N_{E} \\
& \qquad g_{j}(\overline{\boldsymbol{X}}) \geq 0, j=1 \ldots N_{I},
\end{aligned}
$$

where $\overline{\boldsymbol{X}}$ and $F(\overline{\boldsymbol{X}})$ are the vector of free parameters and the cost function, respectively, as defined in part one, and $h_{i}(\overline{\boldsymbol{X}})$ and $g_{j}(\overline{\boldsymbol{X}})$ are two sets of $N_{\mathrm{E}}$ equality and $N_{\mathrm{I}}$ inequality constraints, respectively. In the case of maximum likelihood methods, instead of maximizing the log-likelihood, one can equivalently minimize its negative.

As discussed in the previous section, one possible solution to this constrained function minimization is to add the constraints to the fit (Eq. 60). This approach is equivalent to the method of penalties (Fletcher, 2013), which reformulates the problem as an unconstrained optimization, by adding a penalty term to the cost function $F(\overline{\boldsymbol{X}})$. Thus, the objective becomes minimizing a penalized cost function $F^{\prime}(\overline{\boldsymbol{X}}, \alpha)$ : 


$$
F^{\prime}(\overline{\boldsymbol{X}}, \alpha)=F(\overline{\boldsymbol{X}})+\alpha \times \sum_{i} h_{i}(\overline{\boldsymbol{X}})^{2}+\sum_{j} \beta_{j} \times g_{j}(\overline{\boldsymbol{X}})^{2},
$$

where $\alpha$ and $\beta_{\mathrm{j}}$ are penalty factors with the following properties:

$$
\begin{aligned}
& \alpha>0, \\
& \beta_{j}=\left\{\begin{array}{l}
0 \text { if } g_{j}(\overline{\boldsymbol{X}}) \geq 0 \\
\alpha \text { if } g_{j}(\overline{\boldsymbol{X}})<0
\end{array}\right\},
\end{aligned}
$$

Formulating the $h_{\mathrm{i}}$ and $g_{\mathrm{i}}$ expressions that correspond to any of the equality and inequality constraints above is straightforward. For example, the first two of the $P_{\mathrm{O}}$ constraints given above (Eq. 64) become:

$$
\begin{aligned}
h_{i} & =P_{O}-0.5, \\
g_{i} & =0.4-P_{O} .
\end{aligned}
$$

The gradients of the penalized cost function might be required by the optimization engine. These could be calculated analytically, as follows:

$$
\frac{\partial F^{\prime}(\overline{\boldsymbol{X}}, \alpha)}{\partial \bar{x}_{k}}=\frac{\partial F(\overline{\boldsymbol{X}})}{\partial \bar{x}_{k}}+2 \times\left\{\alpha \times \sum_{i}\left[h_{i}(\overline{\boldsymbol{X}}) \times \frac{\partial h_{i}(\bar{X})}{\partial \bar{x}_{k}}\right]+\sum_{j}\left[\beta_{j} \times g_{j}(\overline{\boldsymbol{X}}) \times \frac{\partial g_{j}(\bar{X})}{\partial \bar{x}_{k}}\right]\right\} .
$$

The derivatives of $h_{\mathrm{i}}$ and $g_{\mathrm{j}}$ with respect to a free parameter $\bar{x}_{k}$ depend on the specific constraints used, and one may need to calculate them using the chain differentiation rule, as in the case of linear constraints. Ultimately, if the constraint functions are too complicated, the gradients can be approximated numerically. Whether the gradients are calculated analytically or numerically, one should keep in mind that inequality penalties are only semi-differentiable and may throw off the optimizer. If this is the case, one possibility is to approximate the penalty into a differentiable function (Bertsekas, 1975). 
The main advantage of the penalty method is that it can be used with any optimization algorithm that was originally designed for non-constrained problems. The main issue, however, is the choice of the penalty parameter $\alpha$. On the one hand, if $\alpha$ is too small, the solution found by the optimizer will be pulled toward $F(\overline{\boldsymbol{X}})$, and then the constraints defined by $h_{i}(\overline{\boldsymbol{X}})$ and $g_{j}(\overline{\boldsymbol{X}})$ may not be exactly satisfied. On the other hand, if $\alpha$ is very large, the solution will satisfy the constraints, in principle. However, the optimizer engine may have a difficult time finding that solution, because the penalized cost function $F^{\prime}(\bar{X}, \alpha)$ may change very abruptly in the $n$-dimensional parameter space. Thus, although it is conceptually and computationally very simple, using the penalized cost function is not exactly a plug-and-play solution, as in the case of linear constraints. To better demonstrate how the fitting procedure is performed $\mathrm{QuB}$, the graphic user interface is described in Fig. 2.4.

A possible strategy is to find the solution iteratively, starting with a relatively small $\alpha$, and increasing it until some convergence criteria are satisfied (Himmelblau, 1972). This is the approach we are taking here, as summarized in Fig. 2.5. Once a model topology is chosen, the workflow starts with defining - if any - the linear parameter constraints and the behavioral constraints, including any other arbitrary parameter constraints. The next step is to define the cost function and the penalized cost function, according to the specific application (macroscopic fitting, single-channel maximum likelihood, etc.). Then, we choose a set of model parameters as the starting point, $\mathbf{K}_{0}$. Mathematically, these parameters do not need to satisfy either set of constraints (parameter or behavioral), but starting as close as possible is recommended. From the initial model parameters $\mathbf{K}_{0}$, 
we then calculate the initial set of free parameters, $\overline{\boldsymbol{X}}_{0}$. Finally, we initialize the penalty parameter $\alpha$ to $\alpha_{0}$, equal to a small positive number. In practice, this value can be chosen so as to make the data and the penalty components of the overall cost function be approximately equal.

Once these quantities are defined and initialized, we start the optimization procedure, which involves two embedded loops, as shown in Fig. 2.5. An outer loop, indexed by $p$, handles the schedule for updating the penalty parameter $\alpha_{p}$ that is used to calculate the penalized cost function $F^{\prime}\left(\overline{\boldsymbol{X}}, \alpha_{p}\right)$, and an inner loop, indexed by $k$, handles model optimization for a given $\alpha_{\mathrm{p}}$. The penalty parameter $\alpha_{\mathrm{p}}$ is progressively increased at each outer loop iteration, to increase the relative weight of the penalty component in $F^{\prime}\left(\overline{\boldsymbol{X}}, \alpha_{p}\right)$. Thus, the behavioral constraints may be only loosely satisfied at the end of the first outer loop iteration, but they will get tighter each time $\alpha_{\mathrm{p}}$ is increased. The outer loop can be run for a predefined number of iterations, or can be terminated when the behavioral constraints are satisfied, if at all possible.

In principle, any type of optimization engine can be used in the inner loop. As explained, the optimizer is completely model- and penalty-blind. Essentially, the optimizer solves an unconstrained minimization problem, operating with a set of free parameters $\overline{\boldsymbol{X}}$. However, as it explores the free parameter space in search for a minimum, the optimizer will require, for a given $\overline{\boldsymbol{X}}_{k}$, the penalized cost function $F^{\prime}\left(\overline{\boldsymbol{X}}, \alpha_{p}\right)$ and possibly its gradients. For this, the transformed model parameters $\mathbf{R}_{\mathrm{k}}$ are calculated from $\overline{\boldsymbol{X}}_{k}$, and then the model parameters $\mathbf{K}_{\mathrm{k}}$ are calculated from $\mathbf{R}_{\mathrm{k}}$, as outlined in Fig. 2.3. The model optimization in the inner loop can be run for a predefined number of iterations, or can be 
terminated when some convergence criteria are satisfied. Typically, convergence requires that there be no substantial changes in the free parameter values and in the cost function (and its gradients be close to zero), from one iteration to the next.

\subsubsection{Testing the algorithm}

To clarify the computational procedures described in both parts of this study, we give a step-by-step numerical example. For illustration purposes, we chose the model shown in

Fig. 2.6A, which is complex enough to accommodate an allosteric factor ( $a_{1}$ in Fig.

2.6A), an external parameter representing the number of active channels in the recording $\left(N_{\mathrm{C}}\right)$, and several parameter and behavioral constraints. At the same time, the model is small enough to allow us to print the vectors and matrices used in the numerical computation. Readers who wish to implement their own code can use these examples for verification. Briefly, we tested the algorithms by fitting a stochastically simulated set of macroscopic data, generated in response to a typical voltage-clamp step protocol. We intentionally chose a relatively small data set (the time course of activation and inactivation at different voltages, and the voltage-dependent steady-state activation and inactivation curves, as shown in Fig. 2.7) to illustrate potential parameter identifiability issues and the effect of constraints. The data were fitted in multiple runs, with each run enforcing a different set of constraints, as outlined in Fig. 2.6B. The simulation, data analysis, and fitting procedures are explained in Materials and Methods.

We define the following set of model parameters $\mathbf{K}$ :

$$
\boldsymbol{K}=\left\{k_{1,2}^{0}, k_{1,2}^{1}, k_{2,1}^{0}, k_{2,1}^{1}, k_{2,3}^{0}, k_{2,3}^{1}, k_{3,2}^{0}, k_{3,2}^{1}, k_{3,4}^{0}, k_{3,4}^{1}, k_{4,3}^{0}, k_{4,3}^{1}, a_{1}, q_{1}\right\},
$$


where $a_{1}$ is the allosteric factor and $q_{1}$ is the channel count. Thus, the model has a total of 14 model parameters. Their numerical values are given in the Methods section. The corresponding vector of transformed model parameters $\mathbf{R}$ is:

$$
\boldsymbol{R}=\left\{\varepsilon_{1,2}^{0}, k_{1,2}^{1}, \varepsilon_{2,1}^{0}, k_{2,1}^{1}, \varepsilon_{2,3}^{0}, k_{2,3}^{1}, \varepsilon_{3,2}^{0}, k_{3,2}^{1}, \varepsilon_{3,4}^{0}, k_{3,4}^{1}, \varepsilon_{4,3}^{0}, k_{4,3}^{1}, \varphi_{1}, \phi_{1}\right\},
$$

where:

$$
\begin{aligned}
& \varepsilon_{\mathrm{ij}}^{0}=\ln k_{\mathrm{ij}}^{0}, \\
& \varphi_{1}=\ln a_{1}, \\
& \phi_{1}=\ln q_{1} .
\end{aligned}
$$

\subsubsection{Applying linear parameter constraints}

The test model has allosteric relationships that require two sets of linear parameter constraints. The first set applies to the forward transitions $C_{1}$ to $C_{2}$ and $C_{2}$ to $C_{3}$ :

$$
k_{1,2}=a_{1} \times k_{2,3}
$$

As explained above, we apply the logarithm on both sides of Eq. 80 and obtain a set of two equality relationships:

$$
\left\{\begin{array}{l}
\ln k_{1,2}^{0}=\ln a_{1}+\ln k_{2,3}^{0} \\
k_{1,2}^{1}=k_{2,3}^{1}
\end{array}\right\} .
$$

The backward transitions $\mathrm{C}_{3}$ to $\mathrm{C}_{2}$ and $\mathrm{C}_{2}$ to $\mathrm{C}_{1}$ have a similar allosteric relationship, which results in another set of two equality relationships:

$$
\left\{\begin{array}{l}
\ln k_{3,2}^{0}=\ln a_{1}+\ln k_{2,1}^{0} \\
k_{3,2}^{1}=k_{2,1}^{1}
\end{array}\right\}
$$


Altogether, we have a set of four linear mathematical relationships between the transformed model parameters in $\mathbf{R}$ :

$$
\left\{\begin{array}{l}
\varepsilon_{1,2}^{0}-\varepsilon_{2,3}^{0}-\phi_{1}=0 \\
\varepsilon_{3,2}^{0}-\varepsilon_{2,1}^{0}-\phi_{1}=0 \\
k_{1,2}^{1}-k_{2,3}^{1}=0 \\
k_{3,2}^{1}-k_{2,1}^{1}=0
\end{array}\right\} .
$$

Together, these four equations reduce the number of free parameters by four, from 14 down to 10 . We must point out that the same allosteric relationships could be implemented just as well without the explicit use of an allosteric factor. Thus, we could write the following constraint equation:

$$
\frac{k_{1,2}}{k_{2,3}}=\frac{k_{3,2}}{k_{2,1}}
$$

Again, after taking the logarithm, we obtain a set of two equations:

$$
\left\{\begin{array}{l}
\ln k_{1,2}^{0}-\ln k_{2,3}^{0}=\ln k_{3,2}^{0}-\ln k_{2,1}^{0} \\
k_{1,2}^{1}-k_{2,3}^{1}=k_{3,2}^{1}-k_{2,1}^{1}
\end{array}\right\} .
$$

The first equation in the set enforces the allosteric relationships at $V=0$. However, the second equation is not sufficient to enforce the allosteric relationships at any voltage. To do so, we must add either one of the following two equations:

$$
\begin{aligned}
& k_{1,2}^{1}=k_{2,3}^{1}, \\
& k_{3,2}^{1}=k_{2,1}^{1} .
\end{aligned}
$$

Altogether, this is equivalent to having a set of three mathematical relationships: 


$$
\left\{\begin{array}{l}
\ln k_{1,2}^{0}-\ln k_{2,3}^{0}=\ln k_{3,2}^{0}-\ln k_{2,1}^{0} \\
k_{1,2}^{1}=k_{2,3}^{1} \\
k_{3,2}^{1}=k_{2,1}^{1}
\end{array}\right\}
$$

with the final form:

$$
\left\{\begin{array}{l}
\varepsilon_{1,2}^{0}+\varepsilon_{2,1}^{0}-\varepsilon_{2,3}^{0}-\varepsilon_{3,2}^{0}=0 \\
k_{1,2}^{1}-k_{2,3}^{1}=0 \\
k_{3,2}^{1}-k_{2,1}^{1}=0
\end{array}\right\} .
$$

This result can be easily verified: the same set of equations can be obtained by eliminating $\varphi_{1}$ between the first two equalities in Eq. 83. Without the explicit use of an allosteric factor, the model would have only 13 model parameters. However, there would be only three constraint equations in that case, which means that the number of free parameters would still be the same: 10 . In conclusion, adding an allosteric factor does not necessarily increase the number of free parameters of a model. Instead, it provides a more intuitive way of formulating the relationships that may exist between rate constants.

Another assumption that we made about our test model is that the $\mathrm{O}_{3}$ to $\mathrm{I}_{4}$ transition has the same voltage sensitivity as the $\mathrm{C}_{2}$ to $\mathrm{O}_{3}$ transition. This results in one mathematical relationship:

(89) $\quad k_{3,4}^{1}-k_{2,3}^{1}=0$.

With this relationship, the number of free parameters is down to 9 . We note that this relationship follows from the actual model parameters used to simulate the data. However, even if the true model parameters were unknown, the savvy investigator would still enforce this constraint, motivated by the shape of the activation curve, which reaches a constant value towards the more positive voltages (Fig. 2.7B). For this particular 
model, this aspect of the activation curve suggests that the rates of activation and inactivation increase by approximately the same factor with voltage. If, for example, the inactivation rate had a stronger voltage dependence than the activation rate $\left(k_{3,4}^{1}>k_{2,3}^{1}\right)$, the activation curve would start turning down at more positive potentials.

The final assumptions we made involve inequality constraints. Thus, we constrained the rate of recovery from inactivation $\left(\mathrm{I}_{4}\right.$ to $\left.\mathrm{O}_{3}\right)$ to have negative voltage dependence, and the deactivation rates $\left(\mathrm{O}_{3}\right.$ to $\mathrm{C}_{2}$ and $\mathrm{C}_{2}$ to $\left.\mathrm{C}_{1}\right)$ to have a voltage sensitivity greater than -0.15 $\mathrm{mV}^{-1}$ :

$$
\begin{aligned}
& k_{4,3}^{1}
\end{aligned}
$$

Since the $k_{3,2}^{1}$ and $k_{2,1}^{1}$ factors are already constrained to be equal, we apply the inequality constraint to $k_{2,1}^{1}$ only, to avoid redundancy. To handle these two inequality constraints, we add two slack variables, $z_{1}$ and $z_{2}$, and write two equality relationships:

$$
\text { (91) } \begin{aligned}
k_{4,3}^{1} & =0.0-z_{1}{ }^{2}, \\
k_{2,1}^{1} & =-0.15+z_{2}{ }^{2} .
\end{aligned}
$$

Thus, although we added two constraints, we also added two slack variables. As a result, the number of free parameters remains the same: 9 .

We summarize here all the constraint equations: 
(92) $\left\{\begin{array}{l}\varepsilon_{1,2}^{0}-\varepsilon_{2,3}^{0}-\phi_{1}=0 \\ \varepsilon_{3,2}^{0}-\varepsilon_{2,1}^{0}-\phi_{1}=0 \\ k_{1,2}^{1}-k_{2,3}^{1}=0 \\ k_{3,2}^{1}-k_{2,1}^{1}=0 \\ k_{3,4}^{1}-k_{2,3}^{1}=0 \\ k_{4,3}^{1}=0.0-z_{1}^{2} \\ k_{2,1}^{1}=-0.15+z_{2}^{2}\end{array}\right\}$

\section{$\underline{\text { 2.6.7 Linear algebra calculations }}$}

We can now formulate the constraint matrix $\mathbf{M}$ and vector $\mathbf{V}$, as in $\mathbf{E q . ~ 3 7 : ~}$

$$
M=
$$

$\begin{array}{lcccccccccccccc} & \varepsilon_{1,2}^{0} & k_{1,2}^{1} & \varepsilon_{2,1}^{0} & k_{2,1}^{1} & \varepsilon_{2,3}^{0} & k_{2,3}^{1} & \varepsilon_{3,2}^{0} & k_{3,2}^{1} & \varepsilon_{3,4}^{0} & k_{3,4}^{1} & \varepsilon_{4,3}^{0} & k_{4,3}^{1} & \varphi_{1} & \phi_{1} \\ (1) & 1 & & & & -1 & & & & & & & & -1 & \\ (2) & & & -1 & & & & 1 & & & & & & -1 \\ (3) & 1 & & & & -1 & & & & & & & & \\ (4) & & & & -1 & & & & 1 & & & & & & \\ (5) & & & & & & -1 & & & & 1 & & & \\ (6) & & & & & & & & & & & 1 & \\ (7) & & & & & & & & & & & & & \end{array}$

$$
\boldsymbol{V}=\begin{gathered}
0 \\
0 \\
0 \\
0 \\
0 \\
0-z_{1}{ }^{2} \\
-0.15+z_{2}{ }^{2}
\end{gathered}
$$


For easier visualization, we only entered the non-zero elements in the $\mathbf{M}$ matrix. As $\mathbf{M}$ contains only constant values, it can now be decomposed with the singular value decomposition technique into three matrices, as in Eq. 40:

\begin{tabular}{|c|c|c|c|c|c|c|c|}
\hline \multirow{7}{*}{ (94) $\boldsymbol{U}_{M}$} & -0.70711 & 0 & 0 & -0.70711 & 0 & 0 & 0 \\
\hline & -0.70711 & 0 & 0 & 0.70711 & 0 & 0 & 0 \\
\hline & 0 & 0.70711 & 0 & 0 & -0.70711 & 0 & 0 \\
\hline & 0 & 0 & 0.85065 & 0 & 0 & 0 & -0.52573 , \\
\hline & 0 & 0.70711 & 0 & 0 & 0.70711 & 0 & 0 \\
\hline & 0 & 0 & 0 & 0 & 0 & 1 & 0 \\
\hline & 0 & 0 & -0.52573 & 0 & 0 & 0 & -0.85065 \\
\hline (95) & $S_{M}=1.41$ & $\begin{array}{l}05 \\
03 \\
21,\end{array}$ & & & & & \\
\hline
\end{tabular}

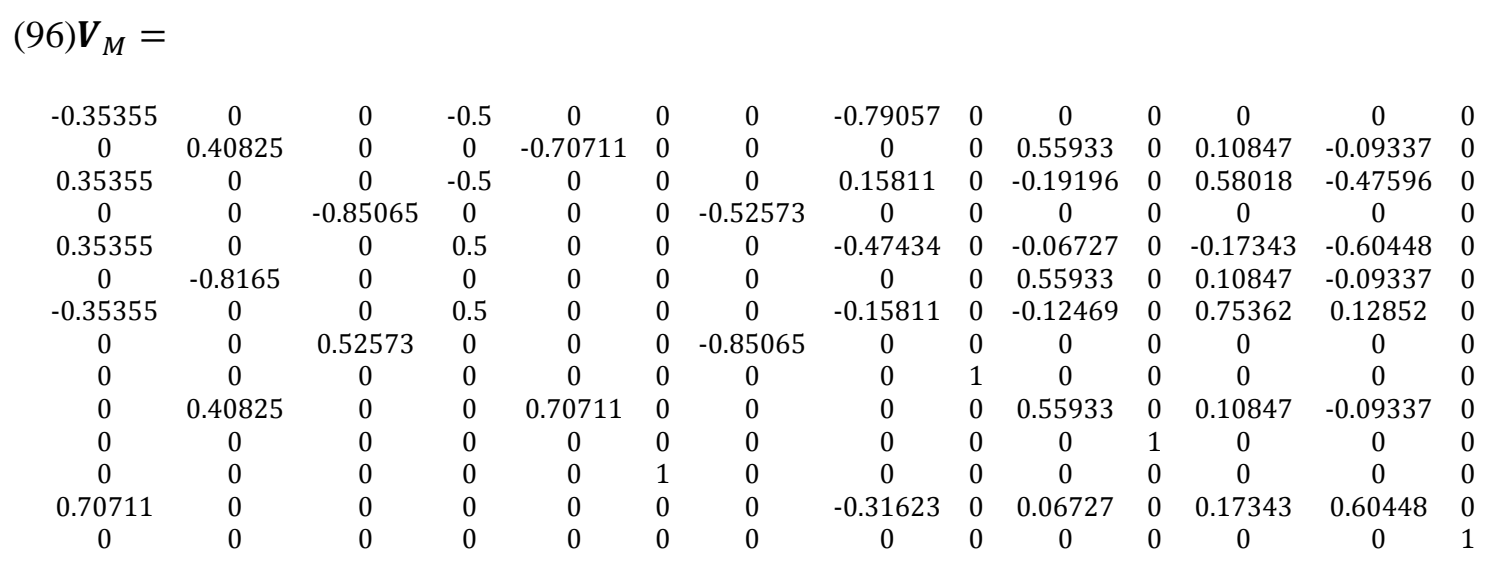

From $\mathbf{V}_{\mathbf{M}}$ we can now obtain the $\mathbf{A}$ matrix, as shown in Eq. 41: 


$\begin{array}{cccccccc}-0.79057 & 0 & 0 & 0 & 0 & 0 & 0 \\ 0 & 0 & 0.55933 & 0 & 0.10847 & -0.09337 & 0 \\ 0.15811 & 0 & -0.19196 & 0 & 0.58018 & -0.47596 & 0 \\ 0 & 0 & 0 & 0 & 0 & 0 & 0 \\ -0.47434 & 0 & -0.06727 & 0 & -0.17343 & -0.60448 & 0 \\ 0 & 0 & 0.55933 & 0 & 0.10847 & -0.09337 & 0 \\ (97) \quad \boldsymbol{A}=-0.15811 & 0 & -0.12469 & 0 & 0.75362 & 0.12852 & 0 \\ 0 & 0 & 0 & 0 & 0 & 0 & 0 \\ 0 & 1 & 0 & 0 & 0 & 0 & 0 \\ 0 & 0 & 0.55933 & 0 & 0.10847 & -0.09337 & 0 \\ 0 & 0 & 0 & 1 & 0 & 0 & 0 \\ 0 & 0 & 0 & 0 & 0 & 0 & 0 \\ -0.31623 & 0 & 0.06727 & 0 & 0.17343 & 0.60448 & 0 \\ 0 & 0 & 0 & 0 & 0 & 0 & 1\end{array}$

The $\mathbf{A}^{-1}$ matrix is simply obtained by transposing $\mathbf{A}$, and we do not show it here. To obtain the $\mathbf{B}$ vector, we must first calculate the pseudo-inverse of $\mathbf{M}, \mathbf{M}^{+}$, as shown in $\mathbf{E q}$. 43. First, we calculate the pseudo-inverse of $\mathbf{S}_{\mathrm{M}}, \mathbf{S}_{\mathrm{M}}{ }^{+}$:

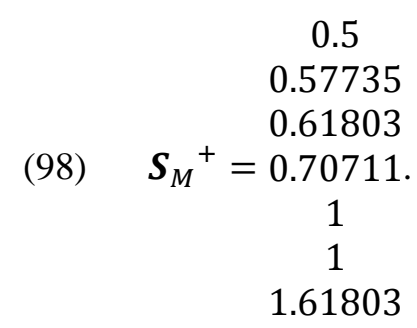

With $\mathbf{S}_{\mathrm{M}}{ }^{+}$, we can calculate $\mathbf{M}^{+}$: 


$\begin{array}{cccccccc}0.375 & -0.125 & 0 & 0 & 0 & 0 & 0 \\ 0 & 0 & 0.66667 & 0 & -0.33333 & 0 & 0 \\ 0.125 & -0.375 & 0 & 0 & 0 & 0 & 0 \\ 0 & 0 & 0 & 0 & 0 & 0 & 1 \\ -0.375 & 0.125 & 0 & 0 & 0 & 0 & 0 \\ 0 & 0 & -0.33333 & 0 & -0.33333 & 0 & 0 \\ (99) \quad M^{+}=-0.125 & 0.375 & 0 & 0 & 0 & 0 & 0 \\ 0 & 0 & 0 & 1 & 0 & 0 & 1 \\ 0 & 0 & 0 & 0 & 0 & 0 & 0 \\ 0 & 0 & -0.33333 & 0 & 0.66667 & 0 & 0 \\ 0 & 0 & 0 & 0 & 0 & 0 & 0 \\ 0 & 0 & 0 & 0 & 0 & 1 & 0 \\ -0.25 & -0.25 & 0 & 0 & 0 & 0 & 0 \\ 0 & 0 & 0 & 0 & 0 & 0 & 0\end{array}$

The $\mathbf{M}^{+}$matrix can now be used to calculate the $\mathbf{B}$ vector, as in Eq. 42. However, when the model contains inequality constraints, the $\mathbf{V}$ vector will contain elements that depend on the slack variables $z_{1}$ and $z_{2}$. During optimization, the slack variables are changed freely by the parameter estimation engine. However, at the beginning of the optimization, they must be initialized by solving their corresponding constraint equation. In this case, $z_{1}$ is initialized as follows:

$$
\begin{aligned}
& \text { (100) } k_{4,3}^{1}=0-z_{1}^{2} \Rightarrow \\
& z_{1}=\sqrt{k_{4,3}^{1}-0}=\sqrt{0.1}=0.31623
\end{aligned}
$$

where 0.1 is the initial value of $k_{4,3}^{1}$. Likewise, $z_{2}$ is initialized as:

$$
\text { (101) } z_{2}=0.27386
$$

With the $z_{1}$ and $z_{2}$ values, we can now calculate the initial $\mathbf{V}$ and $\mathbf{B}$ vectors: 


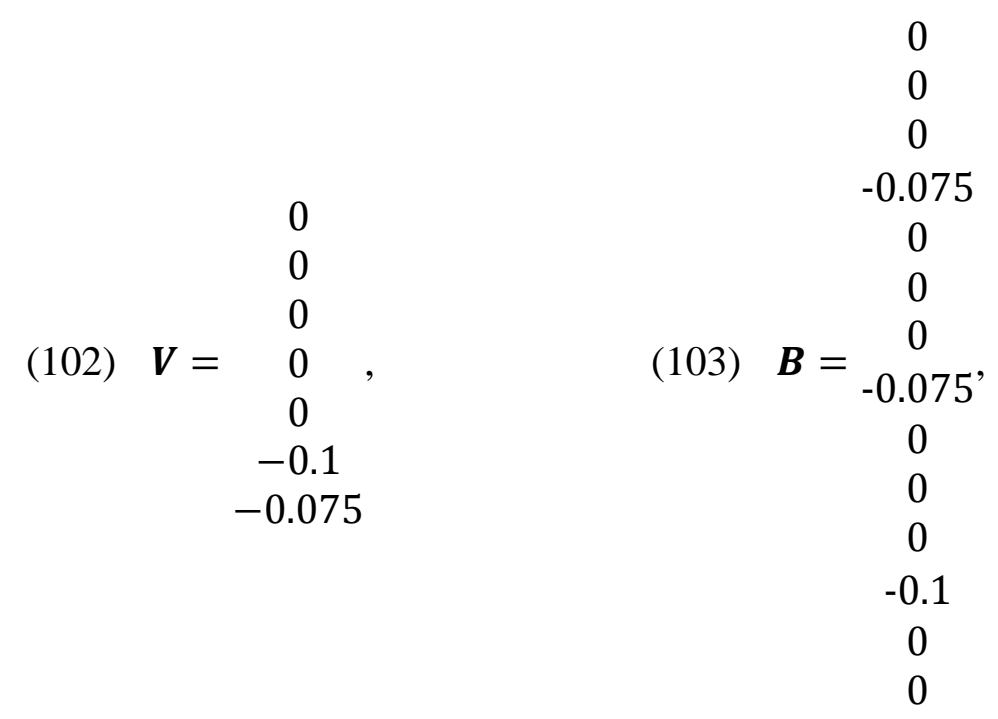

To start the optimization, we must initialize the free parameters $\overline{\boldsymbol{X}}$. When the model constraints include inequalities, as we have here, $\overline{\boldsymbol{X}}$ is formed by the reunion of $\mathbf{X}$ and $\mathbf{Z}$ vectors (Eq. 44). $\mathbf{Z}$ contains the slack variables, which are initialized as shown above, while $\mathbf{X}$ is initialized from the initial set of model parameters $\mathbf{R}^{0}$, using Eq. 39 . Altogether, the initial free parameter values are:

$$
\begin{array}{rc} 
& 10.6402 \\
& 7.3132 \\
& -3.7075 \\
& 2.9957 \\
(104) \quad \overline{\boldsymbol{X}}^{0}= & -4.5367 \\
& -5.6263 \\
& 8.0064 \\
& 0.3162 \\
& 0.2739
\end{array}
$$

Each time the cost function is requested by the optimization engine, the transformed model parameters $\mathbf{R}$ are calculated from the free parameters $\overline{\boldsymbol{X}}$ with Eq. 40. Then, the model parameters $\mathbf{K}$ are calculated from $\mathbf{R}$. 


\subsubsection{Applying arbitrary constraints and behavioral constraints}

In addition to linear parameter constraints, we also tested a few simple but useful constraints that cannot be implemented with the linear algebra formalism. First, we tested an arbitrary parameter constraint that restricts the channel count $N_{\mathrm{C}}$ to a range of values. The test data were simulated with $N_{\mathrm{C}}=5,000$. However, to test the algorithms under more realistic conditions, we enforced a range of values away from the true value $(6,000$ to 8,000$)$. The same strategy was used with all the behavioral constraints introduced next. The constraint and the corresponding cost function component are the following:

(106) $F_{1}^{C}=\beta_{1} \times\left(\frac{N_{C}-6,000}{6,000}\right)^{2}+\beta_{2} \times\left(\frac{8,000-N_{C}}{8,000}\right)^{2}$,

where $\beta_{1}$ and $\beta_{2}$ are numerical factors with the following properties:

$$
\text { (107) } \begin{array}{r}
\left\{\begin{array}{l}
\beta_{1}=0 \text { if } N_{C} \geq 6,000 \\
\beta_{1}=\alpha \text { if } N_{C}<6,000
\end{array}\right\}, \\
\left\{\begin{array}{l}
\beta_{2}=0 \text { if } N_{C} \leq 8,000 \\
\beta_{2}=\alpha \text { if } N_{C}>8,000
\end{array}\right\} .
\end{array}
$$

The normalization to 6,000 or 8,000 makes this penalty component comparable with all the other penalty and data components.

The second is a behavioral constraint that enforces the maximum open probability reached during a brief depolarization step from -120 to $0 \mathrm{mV}$, as illustrated in Fig. 2.6B, Run IV. With the true parameter values, the model predicts a maximum $P_{\mathrm{O}}$ of approximately 0.42 , but we constrained it to 0.5 . The constraint equation and the corresponding cost function component are the following: 


$$
\text { (108) } P_{O}=0.5
$$

$$
F_{2}^{C}=\alpha \times\left(P_{O}-0.5\right)^{2} .
$$

Finally, we tested a behavioral constraint that enforces the time constant of recovery from inactivation. As discussed earlier, it would be rather difficult to calculate this quantity analytically. Instead, we use a surrogate value, extracted from a simulation in response to a two-pulse voltage clamp protocol. As shown in Fig. 2.6B, Run V, we inactivate the channels with a brief voltage pulse, let them recover for $50 \mathrm{~ms}$, and then apply a second pulse to test how many channels have recovered. The recovered fraction is defined as the maximum open probability reached during the second voltage pulse, relative to the first pulse:

$$
\text { (110) } f_{R}=\frac{\left(P_{O}\right)_{\text {pulse2 }}}{\left(P_{O}\right)_{\text {pulse1 }}} \text {. }
$$

Thus, if we want to enforce a specific recovery time constant $\tau_{R}$, we can calculate the corresponding $f_{R}$ for a recovery interval of arbitrary duration $t$, and use that $f_{R}$ value in the behavioral constraint:

$$
\text { (111) } f_{R}=\exp \left(-\frac{t}{\tau_{R}}\right) \text {. }
$$

Our test model predicts a recovered fraction $f_{R}$ of approximately 0.43 with a recovery interval $t=50 \mathrm{~ms}$, at $-80 \mathrm{mV}$, but we constrained it to 0.8 . The constraint equation and the corresponding cost function component are the following:
$f_{R}=0.8$
(113) $F_{3}^{C}=\alpha \times\left(f_{R}-0.8\right)^{2}$.

\subsubsection{Optimizing the model}


We illustrate the performance of the algorithms with six optimization runs, each implementing a different set of constraints, as described in Fig. 2.6B. Together, these examples test the full range of constraints that the algorithms are designed to handle, as they are likely to occur in real life modelling applications: linear equality and inequality parameter constraints, and model behavior and properties. Furthermore, we test all types of model parameters, as defined in the companion paper: rate constant parameters, multiplicative factors $\left(a_{1}\right)$, and external parameters $\left(N_{\mathrm{C}}\right)$. The true parameters of the model, as well as the initial and the estimated parameters obtained in each optimization run, are given in Table 2.1.

In Run I, we enforced only equality linear parameter constraints (Eq. 83). The cost function that was minimized by the optimizer had the following expression:

$$
\text { (114) } F(\overline{\boldsymbol{X}})=F_{1}^{D}+F_{2}^{D}+F_{3}^{D}
$$

where $F_{1}^{D}, F_{2}^{D}$, and $F_{3}^{D}$ are the cost components corresponding to the data shown in Fig. 2.7: time course traces, activation curve, and availability curve, respectively. Each of these data components is the sum of square differences between the data and the prediction of the model, normalized by the total number of data points. The time course component was also normalized to the peak current, as follows:

$$
\text { (115) } F_{1}^{D}=\frac{1}{N_{V} \times N_{t}} \sum_{\mathrm{V}, \mathrm{t}}\left(\frac{y_{\mathrm{V}, \mathrm{t}}-I_{\mathrm{V}, \mathrm{t}}}{y_{\text {Peak }}}\right)^{2}
$$

where $N_{\mathrm{V}}$ is the number of traces, $N_{\mathrm{t}}$ is the number of samples in each trace, $y_{\mathrm{V}, \mathrm{t}}$ and $I_{\mathrm{V}, \mathrm{t}}$ are the data point and the predicted current, respectively, at voltage $V$ and time $t$, and $y_{\text {peak }}$ is the largest negative peak current in the entire data set. With these normalizations, all 
three data cost components take comparable values. We have not done it here but, in principle, one should further normalize the data to account for potentially different levels of noise, such as between the time course traces and the activation and availability curves. One could multiply each cost component by a factor inversely proportional to its normalized variance, to ensure that less noisy data sets will be fit more tightly by the model.

In Run II, we used the same conditions as for Run I, but we added the inequality linear parameter constraints (Eqs. 90-91). In Runs III through VI, we applied the same linear parameter constraints as in Run II, but in each of these we added different constraints that were implemented via the penalty mechanism: an arbitrary parameter constraint that restricts $N_{\mathrm{C}}$ to a range of values (Run III), and behavioral constraints that enforce $P_{\mathrm{O}}$ (Run IV), the recovered fraction $f_{\mathrm{R}}$ (Run V), or both $P_{\mathrm{O}}$ and $f_{\mathrm{R}}$ simultaneously (Run VI). In all these tests, the optimizer minimized a penalized cost function with the following expression:

$$
F^{\prime}(\bar{X}, \alpha)=F_{1}^{D}+F_{2}^{D}+F_{3}^{D}+F^{C}
$$

where $F^{C}$ stands for either $F_{1}^{C}$ (Run III), $F_{2}^{C}$ (Run IV), $F_{3}^{C}$ (Run V), or $F_{2}^{C}+F_{3}^{C}$ (Run VI). The optimization results shown in Fig. 2.8 demonstrate the proper functioning of the algorithm with all types of constraints. To test the convergence of the optimizer, we intentionally chose starting parameters (Table 2.1) that generate prediction curves that deviate substantially from the data, as shown by the blue traces in Fig. 2.3. In all cases, the cost function virtually settled in about 30 iterations (Fig. 2.8A, left panel), after which most model parameters changed little (Fig. 2.8B). For Run I, the final parameter values 
are within approximately $10 \%$ of the true values (Table 2.1), which is to be expected under these conditions (Milescu, 2005). For the other runs, the constraints push some of the parameters away from their true values, as intended. Although the final parameter values (Table 2.1) vary across the six runs, they all predict virtually identical fit curves, all represented by the red traces in Fig. 2.7A/B.

The effect of inequality linear parameter constraints can be observed by comparing Runs I and II. In Run I, the $k_{4,3}^{1}$ parameter is unconstrained and meanders to values as large as $+0.12 \mathrm{mV}^{-1}$, finally converging to a slightly positive value, even though the true value is slightly negative $\left(-0.05 \mathrm{mV}^{-1}\right)$. The convergence to a positive value for $k_{4,3}^{1}$ is not a failure of the search engine, but simply a result of the stochastic nature of the data. In Run II, $k_{4,3}^{1}$ is constrained to a negative range and, as expected, converges to a final value of zero. The convergence to a value that lies on the edge of the constrained range would suggest that this solution is suboptimal, compared to the solution found in Run I. Indeed, the cost function value is nominally larger: 0.000533 for Run II, vs 0.000392 for Run I. The $k_{2,1}^{1}$ parameter is also constrained with an inequality in Run II. However, $k_{2,1}^{1}$ hovers comfortably above its limit in Run I, and, as expected, the constraint applied in Run II has no effect.

In Runs III through VI, the cost function is replaced by a penalized cost function, which adds a penalty function component (Eq. 73). In all of these cases, the penalty function quickly drops by four or five orders of magnitude during the optimization (Fig. 2.8A, right panel). In Run III, where the penalty mechanism enforces a range of values for $N_{\mathrm{C}}$, the penalty function occasionally drops to zero (Fig. 2.8A, right panel, orange trace), 
whenever $N_{\mathrm{C}}$ is within the allowed range and the constraint is exactly satisfied (Fig. 2.9A). Although the initial value of $N_{C}$ was outside the acceptable range $(3,000)$, the optimizer quickly brought $N_{\mathrm{C}}$ within the range, in just a few iterations. We find it interesting that the convergence value of $N_{\mathrm{C}}$ does not lie on the edge of its allowed range $(6,000)$, as close as possible to the convergence value found in Run II $(5,500)$. This suggests the existence of multiple solutions that predict identical fits.

In Runs IV through VI, the penalty mechanism was used to enforce equality relationships for $P_{\mathrm{O}}$ and $f_{\mathrm{R}}$. Like with $N_{\mathrm{C}}$ in Run III, the initial values of $P_{\mathrm{O}}$ and $f_{\mathrm{R}}$ were quite different from their enforced values. However, a few iterations were sufficient to bring $P_{\mathrm{O}}$ or $f_{\mathrm{R}}$ close to their enforced values, as illustrated in Fig. 2.9B and C (green and magenta traces). In contrast to Run III, the penalty function approaches a small value, but does not reach zero (Fig. 2.8A, right panel, green, blue, and violet traces). Accordingly, the enforced quantities hover in a small neighborhood centered on their enforced values (Fig. 2.9B and C). The size of this neighborhood depends on the numerical value of the penalty parameter $\alpha_{p}$ : the larger the $\alpha_{p}$, the smaller the neighborhood. In principle, enforcing the penalty might require several cycles, where each cycle increases the value of $\alpha_{p}$, as illustrated in Fig. 2.5, and tightens the constraint. However, for these relatively simple optimization examples, we initialized the penalty factor as $\alpha_{0}=1$, which enforced the constraints tightly enough in a single penalty cycle.

As expected, adding these constraints that push $P_{\mathrm{O}}$ and $f_{\mathrm{R}}$ away from their true values also results in slightly suboptimal fits in Runs IV through VI, compared to Runs I through III. Furthermore, these constraints expose correlations between properties of the model 
$\left(P_{\mathrm{O}}\right.$ and $\left.f_{\mathrm{R}}\right)$ and certain model parameters. Thus, $P_{\mathrm{O}}$ is inversely correlated with $N_{\mathrm{C}}$. Without any constraint, $P_{\mathrm{O}}$ and $N_{\mathrm{C}}$ are estimated as approximately 0.42 and 5,100, respectively. In contrast, when $P_{\mathrm{O}}$ is constrained (Runs IV and VI), the $N_{\mathrm{C}}$ estimate is lowered to 4,000 (Fig. 2.9A). Vice versa, when $N_{\mathrm{C}}$ is constrained to a larger value, the estimated parameters predict a lower $P_{\mathrm{O}}\left(\right.$ Fig. 2.9B) . Likewise, $f_{\mathrm{R}}$ is correlated with the rate of recovery from inactivation (the $\mathrm{I}_{4}$ to $\mathrm{O}_{3}$ transition). Thus, enforcing $f_{\mathrm{R}}$ to a larger value (0.8) than the true value (0.43) results in a smaller estimate for $k_{4,3}^{0}$ and in a more negative estimate for $k_{4,3}^{1}$ (Fig. 2.8B, Runs V and VI). Considering these potential correlations between different parameters or model properties, one should be careful not to apply contradictory constraints.

\subsection{Discussion}

We have presented here a set of mathematical and computational tools that can be used to estimate kinetic mechanisms that explain new data but also satisfy user-defined constraints or prior knowledge. Fitting a model to a new data set, while accounting for previous data sets is not trivial. Prior knowledge expressed as linear mathematical relationships between two sequential transitions can be easily handled through model parameterization, as the independent parameters are identified by the user and passed on to a search engine, while the remaining (dependent) parameters are simply derived from the first set, whenever necessary. However, a more elegant and flexible solution is the method of reduction (Fletcher, 2013), first applied to kinetic modeling algorithms some twenty years ago (Qin, 1996, 2000a; Milescu, 2005). Yet even the reduction method, despite its reach, is not the universal solution to enforcing prior knowledge. Although 
very powerful, this method is limited to constraints that can be formulated as explicit linear equality relationships between model parameters. Thus, it cannot handle inequalities, nonlinear relationships, and implicit constraints that describe a model property or behavior.

In this study, we proposed a comprehensive set of mathematical and computational tools that address all these limitations and greatly expand the range of prior knowledge that can be enforced. First, we enhanced the reduction method to handle both equality and inequality linear parameter constraints. Furthermore, we expanded the range of parameters that can be constrained, to include not only rate constant parameters, but also allosteric and other similar factors, and external parameters that describe the data or the experiment. Any relationship between these parameters can now be enforced, as long as it is linear. Second, any other types of model constraints, such as range constraints, nonlinear parameter relationships, or model properties and behavior, are handled by applying a penalty to the cost function. Together, the reduction method and the penalty method can handle virtually any type of model constraint that is likely to be encountered in the field.

These methods described here are available through the freely available QuB software, as maintained by our lab. These methods are also easy to implement by interested readers. The only high-level mathematical operation involved is the singular value decomposition, which is readily available from many free linear algebra packages. As illustrated in Fig. 2.3, the code can be implemented as a pair of functions: one that converts a set of interdependent model parameters into a set of free parameters, and a second function that 
performs the reverse operation. The first function is called only once, when the optimization is started, to initialize the free parameters from the model parameters. Any optimization package has one user-customizable callback function that is called each time the search engine needs the cost function for a given set of parameters. The function that converts free parameters into model parameters can be inserted at the beginning of this callback function.

\subsubsection{Compatibility with existing optimization frameworks}

The procedures described here can be easily adapted into a typical optimization package. As illustrated by the workflow diagram in Fig. 2.5, only a few modifications would be required: adding a function that converts between free parameters and model parameters

$\left(\overline{\boldsymbol{X}}_{k} \rightarrow \boldsymbol{K}_{k}\right)$ and vice-versa, modifying the cost-function to calculate and add the penalty, and implementing a schedule to progressively increase the penalty parameter. The first two modifications are trivial, as just about every optimizer will have a callback function where the user writes custom code to calculate the cost function for a given set of free parameters. The third modification is potentially more involved, but a simple solution would be to increase the penalty parameter by hand and restart the optimizer.

\subsubsection{Constrained fitting versus multi-objective fitting}

There is a certain similarity between constrained fitting, on the one hand, and simply including those data that underlie the constraints into a more comprehensive data set to be fitted. The second approach is generally described as multi-objective fitting (Druckmann et al., 2007; Bandyopadhyay and Saha, 2013). While it is not a substitute for the 
reduction method that is used to enforce linear parameter constraints, it could be a substitute for the penalty method. As the name implies, in this case the optimizer would need to find a solution that satisfies multiple objectives, i.e., data sets. This is conceptually equivalent to constrained fitting, but there is also one important difference: in multi-objective fitting, the optimal solution found by the search engine may actually explain poorly each and all of the individual data sets, as long as it is the best overall compromise. Moreover, to find this compromise solution, one must choose a set of weighting factors that encode how much each data set is worth to the model, which is not trivial.

In contrast, the constraining mechanism described in this study will give the highest priority to the constraints and satisfy them exactly (the linear parameter constraints, via the reduction method), or at least very tightly (all other constraints, via the penalty method). Only after the constraints are satisfied, will the model adapt to explain the data, as much as possible. Nevertheless, as explained above, a certain margin of error can be built into the constraints to accommodate noise and potential artifacts, but the constraints will stay tightly within this margin. One advantage to the constraint approach is that one can more easily detect when a model is incompatible with the data. Furthermore, one could also detect inconsistent knowledge, as signaled by incompatible constraints.

\subsubsection{Model behavior: to enforce or not?}

The need for enforcing explicit parameter relationships is obvious, if only to consider microscopic reversibility, or the ratio of sequential activation rates. However, it may be less clear to the reader why model behavior and properties need to be enforced. Why not 
derive them directly from the data? After all, once model parameters are estimated, they can be used to predict any model property or behavior. The problem resides in the potential lack of model and parameter identifiability. In an ideal case, the model would be uniquely identifiable, which means that no other topology exists that can explain the data equally well (Kienker, 1989; Bruno, 2005). Furthermore, the data would be noise and artifact free and the model parameters would be fully identifiable, which means that the model admits a unique solution and the optimizer is able to find it from the data. If this were the case, it would make little sense to enforce a model behavior or property, except to test the sensitivity of the parameters with respect to that behavior.

However, in reality, the true model may never be known, and the working model may be just one out of many equivalent topologies. Furthermore, the parameters may not be fully identifiable, either because the model admits multiple solutions (theoretical parameter identifiability), or because the data are corrupted by noise and artifacts that flatten the cost function surface (practical parameter identifiability) (Milescu, 2005; Raue et al., 2009; Siekmann et al., 2012; Hines et al., 2014; Middendorf and Aldrich, 2017). Thus, estimating the kinetic mechanism from limited data may result in a parameter set that is just one out of many possible solutions, and potentially one with poor predictive power.

This is actually the case with our numerical example: in all runs, the estimates obtained by the optimizer are close to the true values (Table 2.1), except when otherwise constrained (e.g., $N_{\mathrm{C}}$ in Run III). However, the estimates differ across runs, even though the fits are virtually identical between runs and follow closely the data (Fig. 2.7, red lines). How can different sets of parameters produce the same solution? The explanation 
for this apparent contradiction is that the parameters are not uniquely identifiable given the reduced data set. Clearly, adding more constraints or enforcing other model behaviors would improve parameter identifiability and would select only those parameter solutions that are compatible with that behavior. Furthermore, it would also improve model identifiability, making it easier to discover the correct model. Short of the true model, we would at least obtain more robust models. 


\subsection{Figures and figure legends}

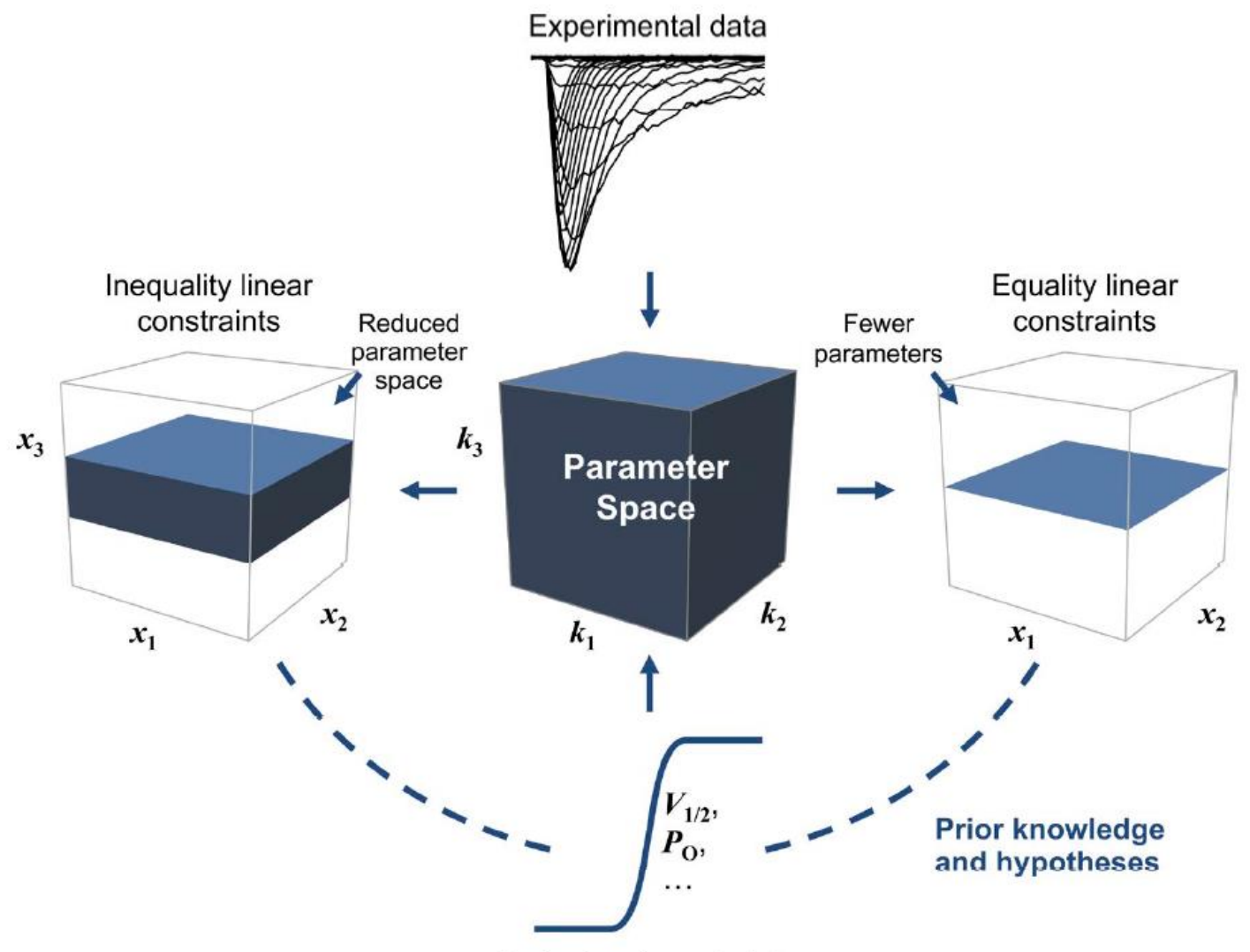

Behavioral constraints

Figure 2.1. Estimating kinetic mechanisms with prior knowledge. A model can be made to fit experimental data while also satisfying user-defined constraints that establish explicit relationships between model parameters, or define specific model behaviors. In the absence of constraints, a fitting algorithm will search a potentially large parameter space, to find a solution that best explains the data. Adding constraints to the model not only enforces prior knowledge but also accelerates the fitting procedure, by narrowing the search space and reducing the number of free parameters. Furthermore, constraints can be used as a mechanism for testing hypotheses against experimental data. 

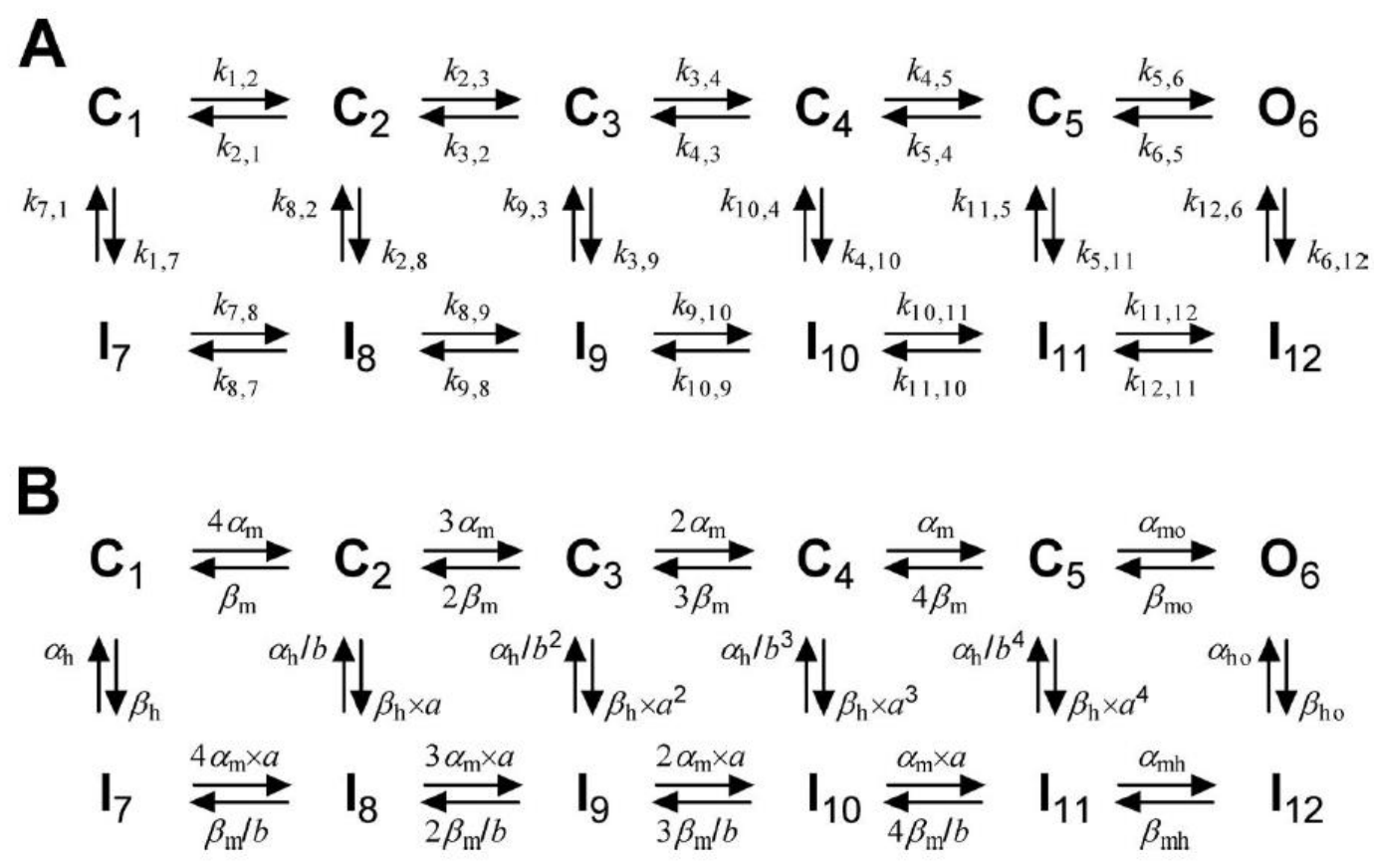

Figure 2.2 Expressing prior knowledge via model topology and parameter relationships. A) an example model that captures the kinetic properties of neuronal sodium channels (Kuo and Bean, 1994; Milescu et al., 2010b). B) various assumptions about the structural and functional elements of the channel are contained in the structure of the model (the states and their connectivity) and in the quantitative relationships between rate constants. The parameter constraints resulting from these assumptions are explained in the text. The $\alpha$ and $\beta$ quantities are voltage-dependent rate constants, while $a$ and $b$ are multiplicative factors expressing allosteric relationships. State labels denote closed (C), closed and inactivated (I), and open (O) states. 

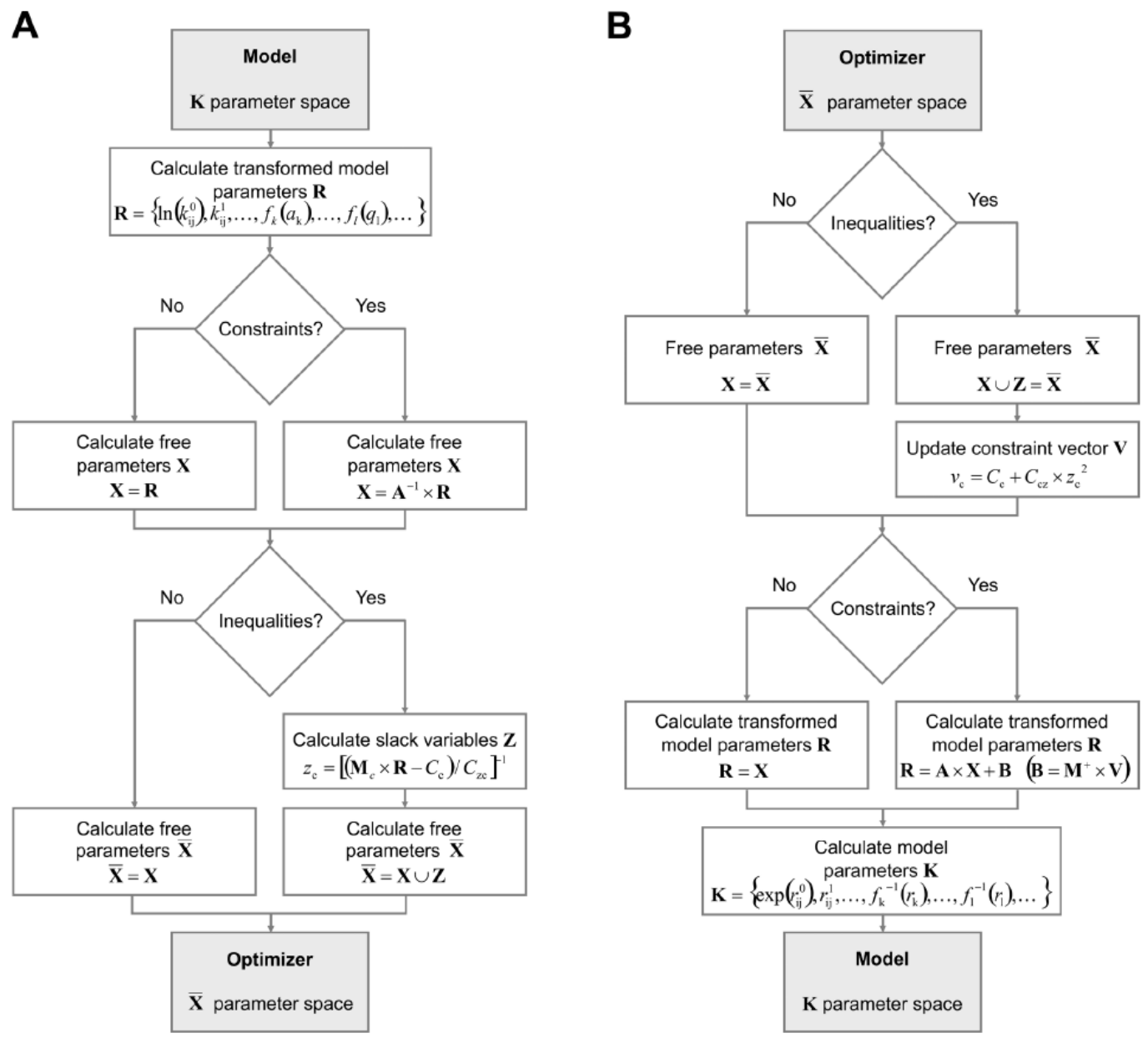

Figure 2.3. Transformations between model parameters and free parameters. The model is defined by a set of interdependent parameters $\mathbf{K}$, whereas prior knowledge is expressed as a set of linear parameter constraints. $\mathbf{K}$ contains pre-exponential and exponential kinetic parameters $\left(k_{i j}{ }^{0}\right.$ and $\left.k_{i j}{ }^{1}\right)$, multiplicative factors $\left(\mathrm{a}_{\mathrm{k}}\right)$, and external parameters $\left(\mathrm{q}_{1}\right)$. To enable more types of constraints, $\mathbf{K}$ is transformed into $\mathbf{R}$ by applying the logarithm or other functions to some of the parameters in $\mathbf{K}$. The linear constraints are reduced via the singular value decomposition to obtain a set of free parameters $\mathbf{X}$. Inequality constraints are handled by a set of slack variables $\mathbf{Z}$. The constraints reduce the number of free parameters in $\mathbf{X}$ by one for each mathematical relationship, although each inequality relationship increases the size of $\mathbf{Z}$ by one. An overall set of free parameters $\overline{\boldsymbol{X}}$ is formed from $\mathbf{X}$ (equality constraints only) or from $\mathbf{X}$ and $\mathbf{Z}$ (equality and inequality constraints). $\overline{\boldsymbol{X}}$ is given to the model-blind optimizer to search for an optimal solution, which can be converted back into a set of model parameters K. A) Conversion from $\mathbf{K}$ to $\overline{\boldsymbol{X}}$. B) Reverse conversion from $\overline{\boldsymbol{X}}$ to $\mathbf{K}$. These conversions can be applied to any kinetic mechanism, regardless of the number of states and connections. All the quantities in the figure are explained in the main text. 


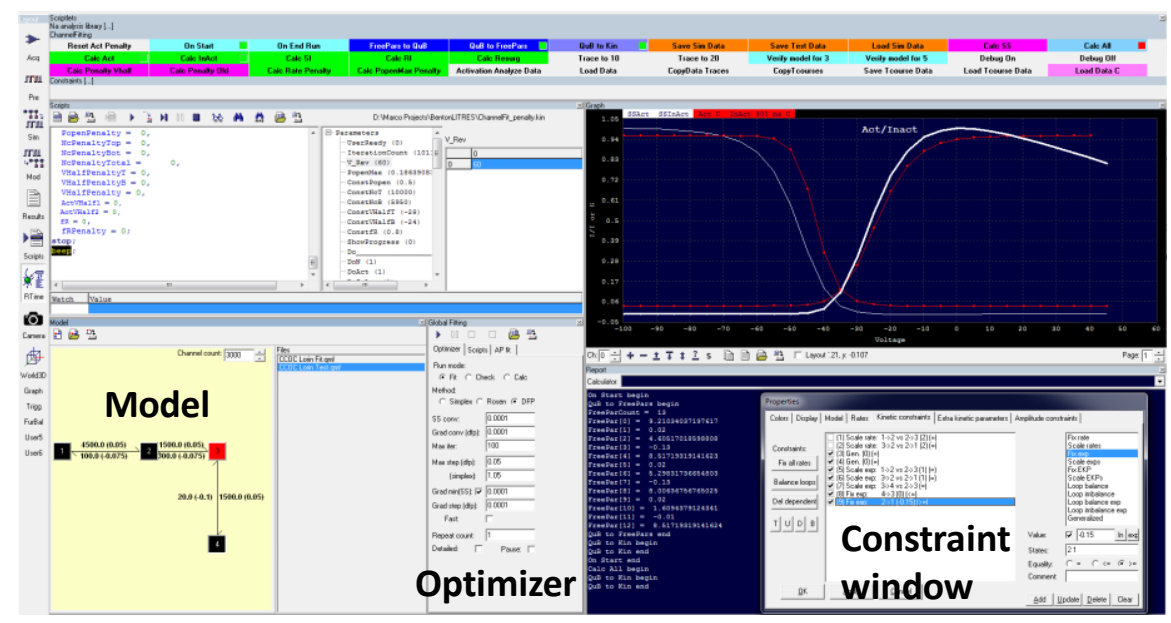

Figure 2.4. Optimizing a model in QuB. The main script is used to declare global variables that are referenced by multiple scriptlets. The main computation is handled by the Optimizer window, which systematically calls individual scriptlets that perform the computation. For each voltageclamp protocol, an individual scriptlet is created to simulate the response of the ion channel model to the same protocol used to collect the data, and also to plot the analyzed data in the Graph window. The optimization process begins by calling a scriptlet that converts the model parameters $(\mathbf{K})$ into a set of free parameters $(\mathbf{X})$. Then, each protocol scriptlet calculates the response of the model to the protocol and plots the data. In this example, the VC data is shown in white and the output of the model in red. After the simulations are generated, the cost-function $(F)$ is calculated between the experimental data and the simulated data, via another scriptlet. The optimizer then uses a gradient descent method to determine a new set of model parameters. The cycle is repeated until convergence, according to a set of criteria. To implement behavioral constraints, another scriptlet is used to calculate the value of the penalty determined by the behavioral constraint. The screenshot also shows the constraint window, where all linear parameter constraints are described for each model. 


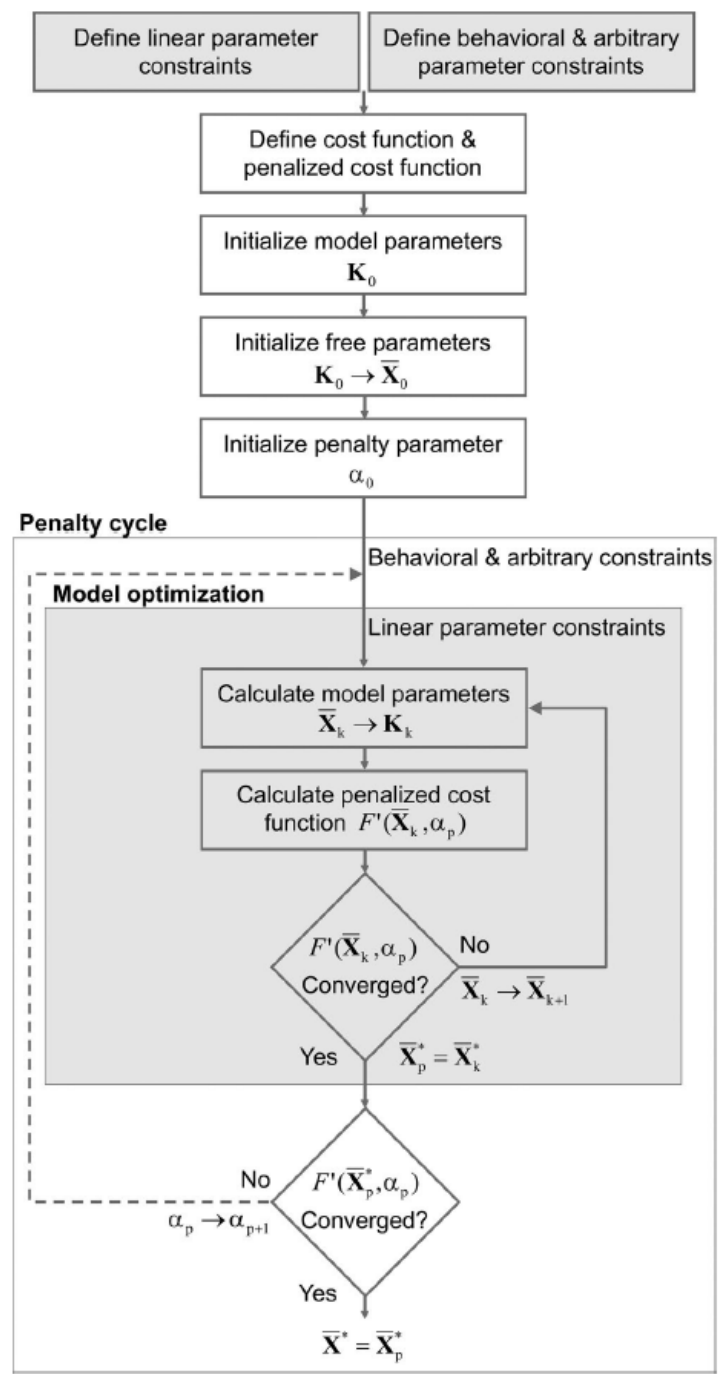

Figure 2.5. Optimizing a constrained model. The flowchart summarizes the computational steps needed to optimize a kinetic model, subject to parameter and behavioral constraints. Linear parameter constraints are implemented via linear algebra transformations between the model parameters $\mathbf{K}$ and the free parameters $\mathbf{X}^{*}$, whereas behavioral constraints or arbitrary parameter relationships are handled by a penalized cost function $F^{\prime}(\mathbf{X} . k, \alpha p)$ that measures the overall error of the model relative to the data and the constraints. The $\mathrm{K} \rightarrow \mathbf{X}^{*}$ and $\mathbf{X}^{*} \rightarrow \mathbf{K}$ transformations are detailed in Fig. 2.3. To calculate the cost function, one needs to generate the response of the model (e.g., probability distributions and macroscopic currents) to the same stimulation protocols used to generate the experimental data and formulate the behavioral constraints. The inner computational loop, indexed by k, optimizes the model for a given penalty factor $\alpha_{p}$, whereas the outer loop, indexed by $p$, gradually increases $\alpha_{p}$, to more tightly satisfy the behavioral and arbitrary parameter constraints. 


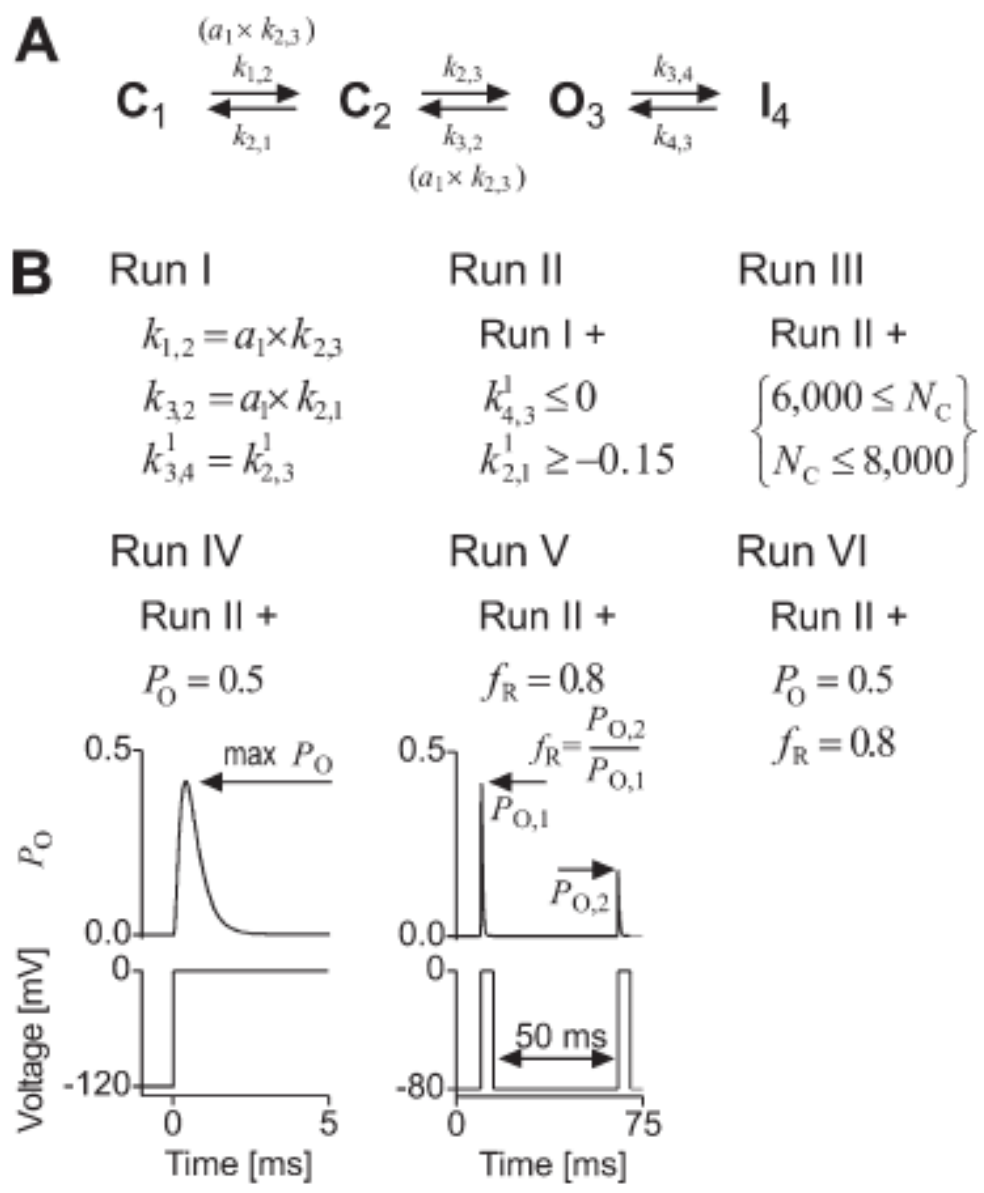

Figure 2.6. Test model with different sets of constraints. A) A simple kinetic mechanism that generates voltage-gated sodium channel-like currents (see Fig. 2.7). All rate constants are as described by Eq. $\left.1 ; k_{i j}=k_{i j}{ }^{0} \times e^{k i j 1 \times V}\right)$; $\mathrm{a}_{1}$ is an allosteric factor, and $\mathrm{N}_{\mathrm{C}}$ is the number of channels. B) Six sets of constraints were applied to the model to test the algorithms (see Figs. 2.8 and 2.9). Runs I and II test linear parameter constraints implemented with linear algebra-based methods that convert model parameters into free parameters, and vice versa. Run I implements only linear relationships, whereas run II adds two inequalities. Runs III through VI test arbitrary parameter constraints and behavioral constraints implemented with the penalty mechanism. Run III tests a parameter range constraint, whereas runs IV through VI test constraints that enforce model properties and behavior: the maximum open probability during a depolarization step $\left(\mathrm{P}_{\mathrm{O}}\right.$, run IV and VI) and the recovered fraction of available channels at $50 \mathrm{~ms}$ after a 5-ms inactivation step $\left(f_{R}\right.$, runs $V$ and $\left.V I\right)$. The $P_{O}$ and $f_{R}$ quantities are obtained as shown below. 

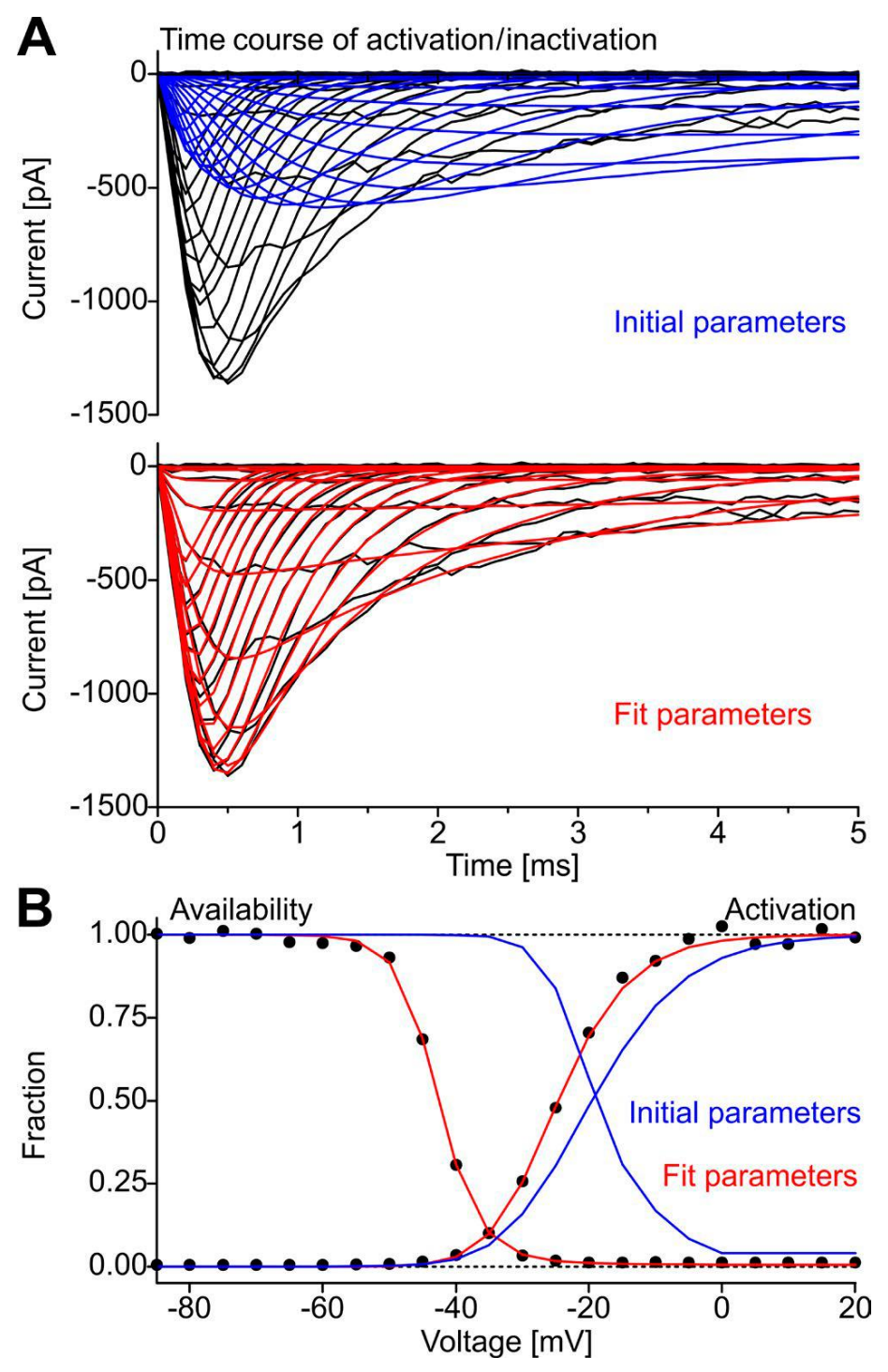

Figure 2.7. Test data and model predictions. A) and B) Whole-cell currents were simulated stochastically with the test model in Fig. 2.6A, using a standard activation/inactivation protocol. The data were processed to extract the time course of activation/inactivation (black traces in A) and the steady-state activation and availability curves (black symbols in B). The time course and steady-state curves were fitted together (see Fig. 2.8). The predictions of the model at the beginning and at the end of optimization are shown by the blue and red traces, respectively. The fit curves correspond to run I in Fig. 2.6B, but all runs resulted in virtually identical fits. The true, initial, and estimated parameters and properties of the model are shown in Table 2.1. 


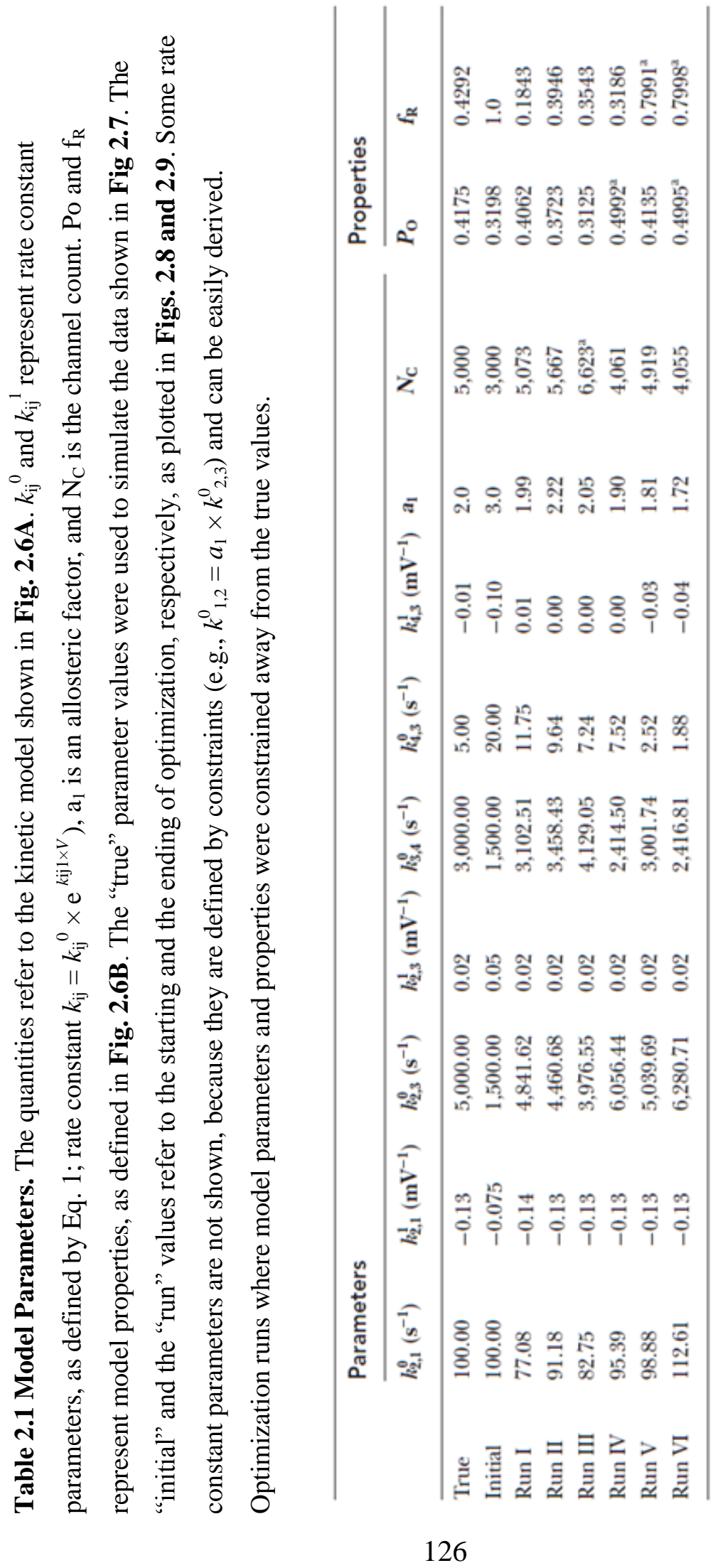



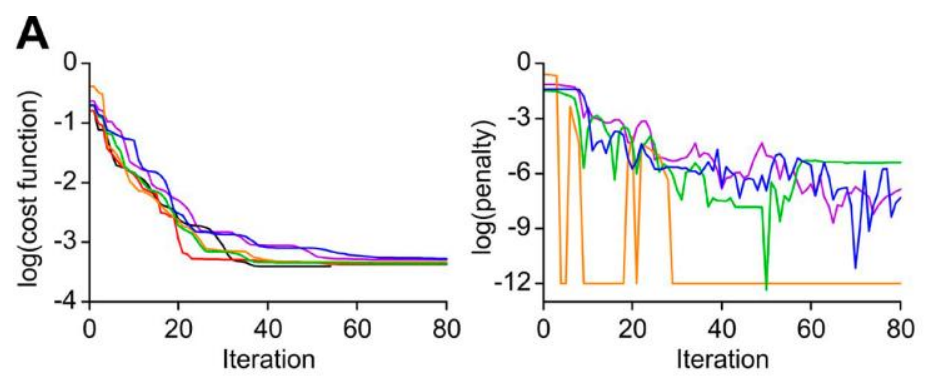

$$
\begin{aligned}
& \text { - Run I } \\
& \text { - Run II } \\
& \text { - Run III } \\
& \text { - Run IV } \\
& \text { - Run V } \\
& \text { - Run VI }
\end{aligned}
$$
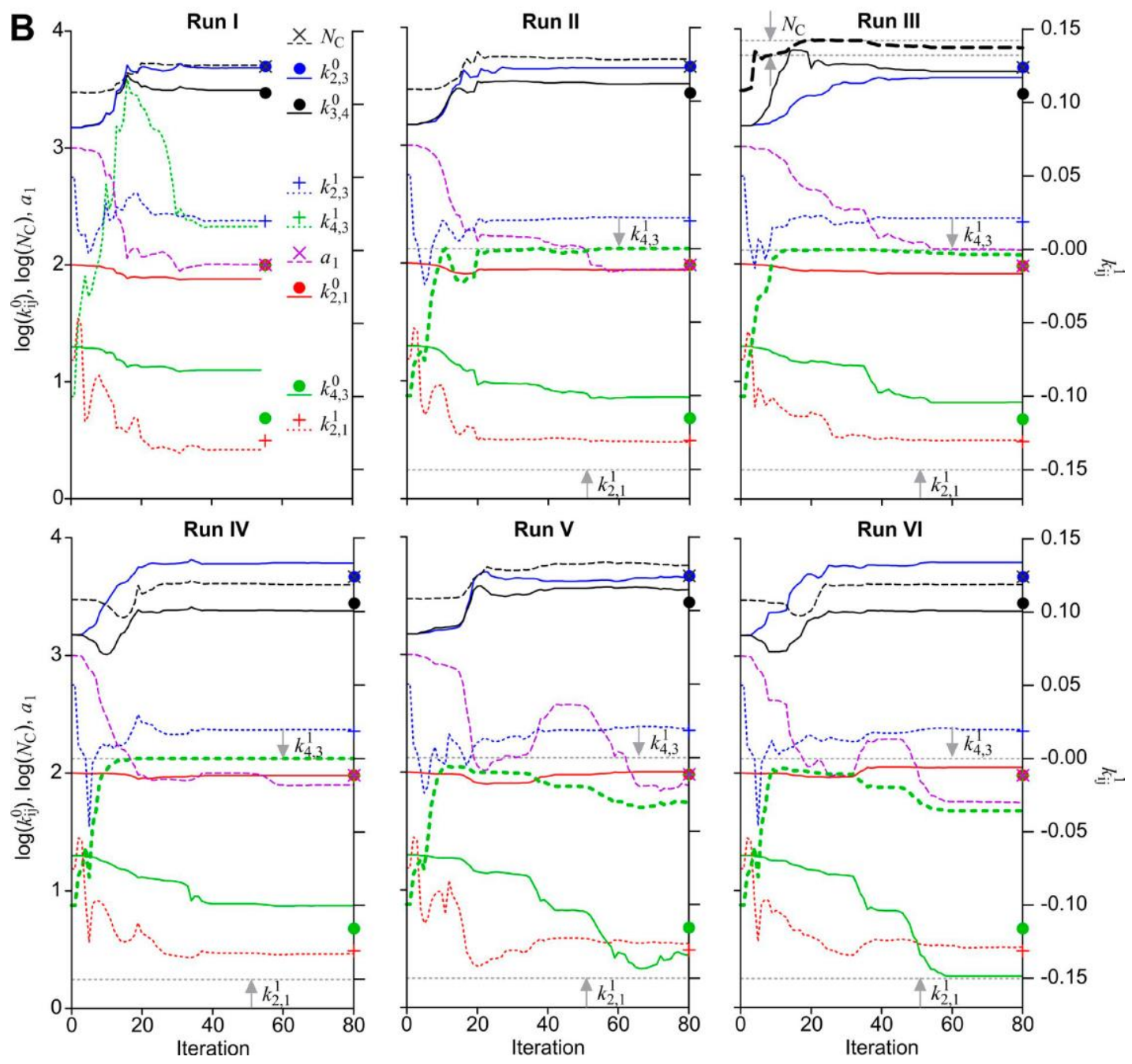

Figure 2.8. Testing optimization with model constraints. The model shown in Fig. 2.6A was optimized to fit the data in Fig. 2.7 (time course and steady-state curves), subject to the six sets of constraints shown in Fig. 2.6B. A) The convergence of the overall cost function (left) and penalty component (right). B) Parameter convergence in each of the six test runs. Only the model parameters $\mathbf{K}$ are shown, but note that the optimizer searches in the free parameter space defined by $\overline{\boldsymbol{X}}$. To reduce clutter, some model parameters are not displayed, as they are defined by constraints (e.g., $k_{1,2}^{0}=a \times k_{2,3}^{0}$ ). For better visualization, the exponential factors $k_{i j}^{1}$ are plotted 
on the right axis (dotted lines), whereas all the other quantities are on the left axis: preexponential factors $k_{i j}^{0}$ (log scale, solid lines), channel count $\mathrm{N}_{\mathrm{C}}(\log$ scale, dashed black line), and allosteric factor $\mathrm{a}_{1}$ (dashed magenta line). The dashed gray horizontal lines and arrows indicate the boundaries of inequality linear constraints for $k_{2,1}^{1}$ and $k_{4,3}^{1}$ (runs II through IV) and the boundaries of the range constraint for NC (run III). Note how $k_{4,3}^{l}$ is estimated as a positive value in run I, but it remains less than zero under the inequality constraint in runs II through VI. In each panel, the symbols aligned with the last iteration mark the true parameter values. 

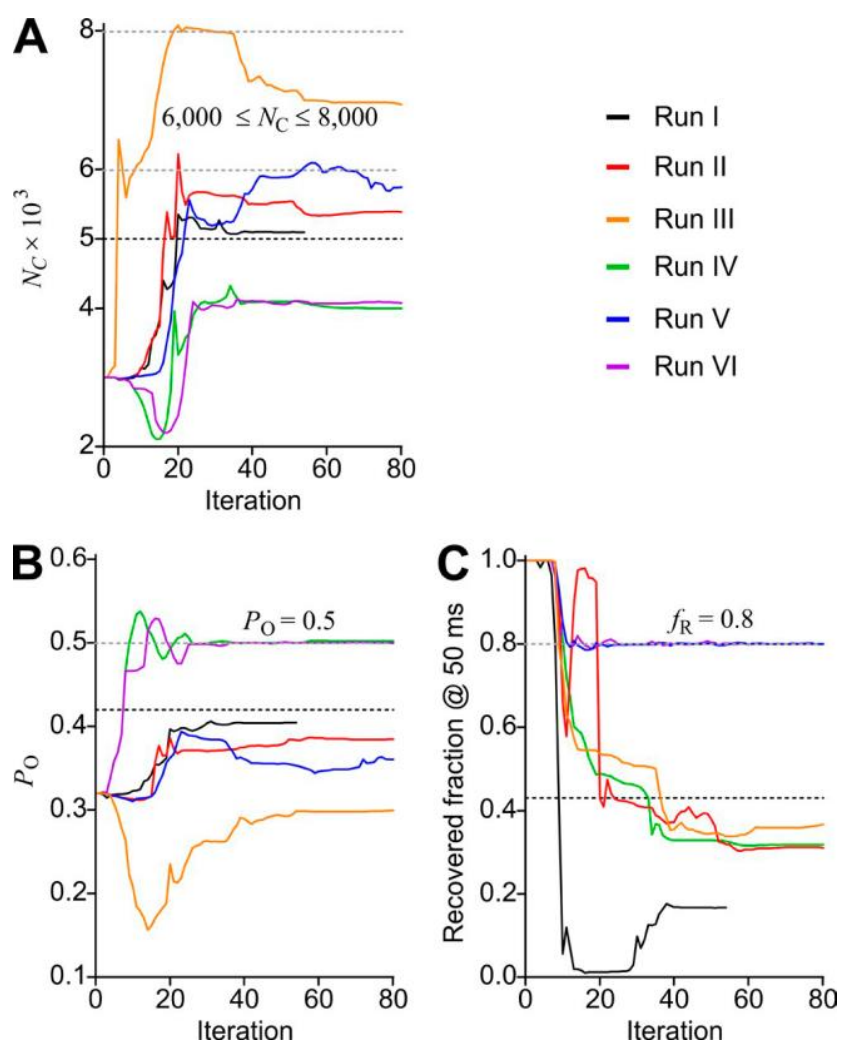

Figure 2.9. Enforcing model properties and behavior. A-C) The convergence of the $\mathrm{N}_{C}$ estimate $(A)$ and calculated $P_{O}(B)$ and $f_{R}(C)$ quantities are shown for each of the six optimization runs described in Fig. 6B. The constrained and the true values are indicated by the gray and black dashed lines, respectively. As expected, $\mathrm{N}_{\mathrm{C}}$ and $\mathrm{P}_{\mathrm{O}}$ are inversely correlated; when one is constrained to be larger or smaller than the true value, the other one becomes smaller or larger, respectively. Likewise, $f_{R}$ is correlated with the rate of recovery from inactivation; when $f_{R}$ is constrained to be larger than the true value, the $\mathrm{k}_{4,3}^{0}$ and $\mathrm{k}_{4,3}^{1}$ estimates become smaller and more negative, respectively. All the enforced quantities quickly reach their enforced range (NC) or value ( $\mathrm{PO}$ and $\mathrm{fR}$ ). Note that without being constrained, $\mathrm{f}_{\mathrm{R}}$ is not well defined by the test data and does not converge to the true value. In contrast, $\mathrm{N}_{\mathrm{C}}$ and $\mathrm{P}_{\mathrm{O}}$ are well defined. 


\title{
Chapter 3
}

\section{D data mapping and real-time experiment control and visualization in brain slices}

This chapter has been adapted from:

Navarro, M.A., J.V.K. Hibbard, M.E. Miller, T.W. Nivin, and L.S. Milescu. 2015. 3D

data mapping and real-time experiment control and visualization in brain slices. Biophys

J. 109:1521-1527. doi: 10.1016/j.bpj.2015.08.045.

\begin{abstract}
We propose here two basic concepts that can streamline electrophysiology and imaging experiments in brain slices and enhance data collection and analysis. The first idea is to interface the experiment with a software environment that provides a $3 \mathrm{D}$ scene viewer where the experimental rig, the brain slice, and the recorded data are represented to scale. Within the 3D scene viewer, the user can visualize a live image of the sample and 3D renderings of the recording electrodes, with real-time position feedback. Furthermore, the user can control the instruments and visualize their status in real-time, and can play back previously executed experiments and run simulations. The second idea is to integrate multiple types of experimental data into a spatial and temporal map of the brain slice. These data can consist of low-magnification maps of the entire brain slice, for spatial context, and any other types of high-resolution structural and functional images, together with time-resolved electrical and optical signals. The entire data collection can be visualized within the $3 \mathrm{D}$ scene viewer. These concepts can be applied to any other types
\end{abstract}


of experiments, where high-resolution data are recorded within a larger sample, at different spatial and temporal coordinates.

Author contribution: M.A.N. wrote scripts to implement new objects and scenes within the $3 \mathrm{D}$ viewer, analyzed imaging data, debugged calibration procedures, designed and performed experiments, created figures, and wrote the manuscript.

\subsection{Introduction}

Brain slices are reduced but powerful preparations, where molecules and individual cells can be studied in the broader context of functional neuronal networks and circuits (Llinás and Sugimori, 1980; Edwards et al., 1989; Smith et al., 1991). Structurally or functionally, each level of organization is best approached with specialized techniques, such as patch-clamp for ion channel kinetics (Hamill et al., 1981; Kole, 2008) or multiphoton microscopy for circuit organization (Sabatini et al., 2002; Helmchen and Denk, 2005; Nakahata and Yasuda, 2018). Combining multiple techniques and data types maximizes the information extracted from each brain slice, for a more comprehensive, multiscale understanding of the entire preparation (Packer et al., 2015). In many studies, however, readily available data are not collected. Potentially useful images are not always recorded in patch-clamp studies, even when they are performed under visual guidance with a microscopy camera.

A difficulty with these experiments is that brain slices can be as large as several millimeters across, and as thick as several hundred microns, or even more. With a lowmagnification imaging objective, the field of view (FOV) can cover much, if not all, of the sample. However, most electrophysiology and many imaging experiments are 
performed under high magnification, where individual neurons can be resolved. Under these conditions, only a small section of the entire slice is seen in the FOV, which makes it difficult to navigate large samples. Having some visual feedback to help place the live image in the 3D sample space would be extremely useful. Yet to our knowledge, this functionality is missing from existing imaging software.

We propose here two basic concepts that can streamline experiment execution and enhance data collection and analysis. The first idea is to interface the experiment with a 3D virtual reality software environment to provide an immersive experience to the investigator. Much as a 3D computer game makes the player feel in the midst of the action on the screen, this environment would bring the user into a 3D virtual world that represents to scale the experimental rig, the brain slice, and the recorded data. By providing a two-way link to experiments, the program would allow the user to control the instruments but also to visualize their status in real-time. Furthermore, the same virtual environment could be used to revisit previously executed experiments and to run simulations.

The second idea is to integrate multiple types of experimental data into a spatial and temporal map of the brain slice. For example, a low-resolution map of the entire brain slice could be obtained at low-magnification with IR-Differential Interference Contrast (IR-DIC) or epifluorescence. This map would provide context and serve as a guide for further investigation at higher resolution. Areas of interest or individual neurons can be identified and examined with optical or electrical methods. The resulting images and signals would be embedded and visualized on the map, with their spatial and temporal 
coordinates recorded. These data may include, for example, multi-photon z-stacks, timeresolved calcium fluorescence, or patch-clamp recordings. We have implemented these concepts in the freely available QuB software package, which can be downloaded at http://milesculabs.biology.missouri.edu/QuB.html.

\subsection{Methodology}

\subsubsection{Animal procedures and brain slice electrophysiology}

All animal procedures were approved by the University of Missouri Animal Care and Use Committee. To minimize the use of animals, the software was tested during experiments performed for other projects in the lab. The brain slices used here for illustration were obtained from Sprague Dawley and TH-GFP-labeled rats (Taconic, USA), following standard slice preparation and electrophysiology procedures.

\subsubsection{Imaging hardware}

We used a customized upright microscope (Scientifica, UK) equipped with Dodt contrast, epifluorescence, and two-photon imaging with a MaiTai HP laser (Newport, USA), and with computer-controlled motorized positioners (Scientifica, UK) for the translation stage, objective, and recording electrodes. Wide-field imaging was done with a Hamamatsu sCMOS Flash4.0 V2 camera (Hamamatsu Photonics KK, Japan).

\subsubsection{Computer software and hardware}

The QuB program was used for 3D data mapping and experiment control and visualization, and for image acquisition with the camera. ScanImage 3.8.1 (Vidrio 
Technologies, USA) was used for two-photon image acquisition. PatchMaster (HEKA, Germany) and QuB were used for patch-clamp and data acquisition. All computer work was done on a dual-processor workstation equipped with 8-core Opteron 4284 processors (AMD, USA) and an R9 280X Radeon video card (AMD, USA), running Windows 7 (Microsoft, USA).

\subsubsection{Software platform}

To rapidly design, implement, and test the concepts and computational tools described here, we took advantage of the QuB software (Milescu, 2000-2006; 2015). QuB is an open-source platform, featuring algorithms and functionality that have been applied to a broad range of problems, from ion channel biophysics (Qin, 1996; Milescu, 2005) to neuronal physiology (Milescu et al., 2008; Milescu et al., 2010b). QuB also features a powerful scripting language (KPL) that we had previously developed and now have expanded, which provides high-level functions to record and process data, and to simulate and estimate ion channel, neuronal, and network models.

\section{$\underline{3.2 .53 \mathrm{D} \text { visualization }}$}

To handle the 3D visualization of data and experiments, we have added a 3D scene viewer to the $\mathrm{QuB}$ program, based on the glscene library. The $3 \mathrm{D}$ scene can be interactively navigated with a computer mouse for an arbitrary point of view with the viewer camera. The scene can also be displayed for stereoscopic visualization using virtual reality glasses (Occulus Rift). Two new classes of computational objects were designed and added to the KPL scripting language. The first class, which we call 
"world3d", is used to represent data (images, electrical recordings, etc.), equipment (objective, imaging camera, isolation table, laser, etc.), or any visual or non-visual type of object. The world $3 d$ objects can be created and organized hierarchically, to capture the structural and functional relationships between the objects that they represent. The second class, which we call "globject" for graphics library object, implements a collection of building blocks for 3D graphics. Each world3d object can use one or more globjects to render its visual content in the 3D scene viewer. Multiple types of globjects are available, where each type implements a 3D graphics primitive, such as lines, cylinders, etc., and can be customized in terms of size, color, position, etc. An example of what can be created with these computational objects is shown in Fig. 3.1.

In addition to the base world $3 d$ object type, there are several derived types that have special meaning and functionality. For example, there are objects that handle the acquired images and other types of data, display the live image coming from the camera or the laser scanner, or control the positioning motors. Some special objects are automatically created by the software (predefined), such as the "liveview" discussed below. The user can create additional objects via the KPL scripts, based on any of the existing types. Whether predefined or user-defined, all these objects can be fully customized and manipulated individually (resized, translated, rotated, etc.), while their collection of globjects can be modified or expanded. These changes can be applied interactively or via the scripting language. The scene can also be animated for instructional purposes. For example, one could demonstrate microscope function by animating the light path 
corresponding to different imaging modes, such as two-photon vs. epifluorescence, as illustrated in Fig. 3.1B and C.

\subsection{Real-time 3D visualization and control of experiments}

\subsubsection{Live imaging}

We implemented a simple solution that allows the investigator to see a live image and simultaneously visualize its location within the sample space (Fig. 3.2). For this, we included in the 3D scene a specialized world3d object, called the "liveview", that displays the live image coming from the video source (camera or laser scanner), as seen through the imaging objective. This liveview object is a representation to scale of the field of view (FOV), with its size depending on the currently selected objective. As the FOV is moved during an experiment, by translating the objective along the $\mathrm{z}$-axis, the liveview follows automatically, repositioning itself in the virtual 3D space, providing real-time navigational feedback to the user. With our experimental setup, we were able to achieve update rates of the liveview content of at least 60 frames per second, ensuring fast and smooth video.

Very importantly, a low-resolution image of the entire brain slice can be recorded and shown throughout the experiment as a background map, to provide spatial context to the liveview (Fig. 3.2). Additional graphical elements are also drawn in the scene viewer, to help the user understand how deep the solution is in the chamber, how thick the slice is, 
and where the FOV is located within the slice or solution (Fig. 3.2). The coordinates for solution and slice surface are approximate values that can be determined by the user.

The live imaging functionality in QuB is not only useful for knowing the location of the FOV at all times but can also be used to analyze imaging data. For example, in the case of functional imaging i.e., using fluorescent calcium or voltage sensitive dyes, an ROI can be placed within the camera viewport to calculate the average pixel intensity in realtime. Taking advantage of QuB's data acquisition functionality, originally developed to implement dynamic clamp, the KPL was modified to add functions that can find the average pixel intensity or standard deviation of multiple ROIs. Currently, square ROIs can simply be placed within the camera window and the real-time engine can perform the computation and plot the data over time. By recording the output of multiple ROIs, one can also perform online background subtraction to find the change in fluorescence online $(\Delta \mathrm{f} / \mathrm{f})$. This allows the experimenter to have real-time information on the status of the experiment. As the data is processed in real-time, the recording of each individual frame does not necessarily need to be recorded, drastically reducing file size for very long acquisitions. However, since the actual images are discarded, it prevents the experimenter from being able to go back and re-process the data to account for movement artifacts or changes in the focal plane. This functionality is demonstrated in Fig. 3.3, showing a screen shot of QuB during calcium imaging experiments.

\subsubsection{Motorized positioning}

Motorized positioners are commonly used to translate the imaging objective along the zaxis, to translate the recording chamber or the entire microscope in the xy-plane, and to 
move the recording electrodes in $\mathrm{x}, \mathrm{y}$, and $\mathrm{z}$. To handle the motorized positioning operations, we created a specialized type of world3d object that establishes a two-way communication with the motor controllers. Interactively or programmatically, these "positioner" objects send commands to the motor controllers to read or set the 3D coordinates of the positioned objects, and update the 3D scene accordingly. We use several positioner objects: one for the FOV, called "viewport", and one for each of the recording electrodes, called "pipettes".

The viewport is a non-visual object that is linked to the motors that move the objective and the translation stage (or the entire microscope). With our upright microscope configuration, when these motors move the translation stage, the sample is physically moved, while the objective or FOV remains in position. However, it makes more sense conceptually to take the opposite view, and consider that the sample remains stationary, while the FOV is moved by the motors. Thus, the viewport can be conveniently used as an interface to position the liveview and image the sample at specific 3D coordinates. When its motors move, the viewport updates the position in the $3 \mathrm{D}$ scene of all its associated world3d objects, including the liveview, which will update its live content as well. When a different section of the sample needs to be imaged, the user simply changes the position of the viewport in the 3D scene, which then sends the appropriate commands to the objective and translation stage motors.

The pipette avatars visually represent the recording electrodes in the $3 \mathrm{D}$ scene and are linked to the motors that move them. As with the liveview, having real-time visual feedback on pipette position is extremely useful: it tells the investigator where the tip of 
the pipette is located relative to the sample, objective, the surface of the perfusion solution, or the bottom of the chamber. Without this visual feedback, the user is oftentimes forced to switch to the low-magnification objective, to be able to safely bring the pipette back in the FOV. This makes it difficult to navigate in the slice over long distances, when the pipette is in the bath or not within the FOV.

Being able to control and set the location of the motors, we developed a semi-automatic targeting procedure that utilizes the projection of the approach axis to determine the necessary location the pipette needs to be placed to successfully approach a desired target cell (Fig. 3.4). This has been extremely useful in being able to reliably target and patch neurons located deep in the slice and reduces the necessary skill needed to patch a cell, which has hastened the training for new patch clampers.

\subsubsection{Calibrating the motorized positioners}

In order to calibrate the system, global Cartesian coordinates of the 3D scene must be defined. We define the $(\mathrm{x}, \mathrm{y})$ origin of the coordinate system as the optical axis of the imaging objective, and the $\mathrm{z}$ origin as the top of the perfusion chamber base for live experiments, or the top of the slide for fixed preparations. In Fig. 3.1, the x-, y-, and zaxes are shown by dashed lines, in red, green, and blue color, respectively.

Ideally, the motors used in the experiment to position the objective, the translation stage, the pipettes, the laser scanning mirrors, and so forth, would all align with the global coordinates. In practice, this is not the case. Typically, experimental rig equipped for laser scanning imaging has the microscope body bolted to the vibration isolation table. 
The imaging objective translates along the z-axis of the microscope body to change the focal plane. The imaging camera and the laser scanning mirrors are attached onto the microscope body. A translation stage is bolted on the isolation table, carrying and moving the recording chamber, the sample, and the electrode positioners in the xy-plane. The electrode positioners move the pipettes along three or four axes (e.g., three physical Cartesian axes and a fourth virtual axis along the pipette length).

All these components may be misaligned with respect to each other, on any or all axes. For example, a common misalignment is the rotation of the camera sensor around the zaxis, relative to the translation stage or when the xy-planes of the translation stage and the camera sensor are not parallel, as illustrated in Fig. 3.5.

These various misalignments must be corrected, as much as possible, in the hardware. Whatever misalignment remains can be reduced by applying software corrections to the positioner coordinates. One could implement rather extensive corrections, but here we will keep it simple. We take as the reference the sensor of the camera, since it is immobile (unlike the camera, the laser scanning plane can be rotated via the command electronics). Hence, the axes of the global coordinate system are defined by the rows and columns of pixels on the camera sensor (the $\mathrm{x}$ - and $\mathrm{y}$-axes, respectively) and by the normal to the sensor plane (the z-axis). Everything else will be corrected relative to this global coordinate system.

The rotation of the translation stage relative to the camera sensor can be simply corrected by applying a mathematical transformation between the coordinates of the virtual viewport object and the coordinates of the physical stage. Unfortunately, a gradient 
between the xy-planes of the sensor and the translation stage cannot be corrected mathematically. As a result of this gradient, a given point in the sample will not maintain focus when the stage is translated, because its image will project on an area of the sensor that can be farther or closer along the z-axis. However, the recorded images can be shown with this tilt, and will still align when displayed in transparency mode. Furthermore, this misalignment does not accumulate along the $\mathrm{x}$ - and $\mathrm{y}$-axes. When tiling images over a large area, the effects of misalignment can be reduced by cropping the sensor when imaging with the camera, or by increasing the zoom factor when using the laser scanning system.

Since the electrode positioners are fixed onto the translation stage, they must be corrected only for misalignment relative to the local coordinate system of the translation stage, not relative to the global coordinates. If we assume that the xy-planes of the electrode positioners and the translation stage are reasonably parallel, the only correction is for rotation around the z-axis. This can be done in the same way as for the stage.

The calibrations described so far must be done only once, when the experimental system is built, or every time a mechanical component is manually repositioned. However, another calibration is necessary each time the recording electrode is replaced with a new one. Pulled glass electrodes can differ significantly in length, and the tip may also be randomly off-center relative to the pipette long axis. By bringing the pipette tip in focus within the live image, its position can be easily extracted in relation to the FOV, which has known coordinates. 
Other useful corrections can also be easily implemented in software. For example, when low and high magnification objectives are used for imaging, they may be misaligned in the xy-plane, or on the z-axis. Taking one objective as reference, the other one can be easily corrected by applying an $(\mathrm{x}, \mathrm{y}, \mathrm{z})$ offset to the motors that position the translation stage and focus the objective. Another calibration is the alignment between the FOV of the camera and the laser scanning system. Again, the camera sensor can be taken as reference, while its own FOV can be calibrated with a graded microscopy scale, or simply calculated from the sensor size and the objective magnification factor.

Most of the mathematical operations for 3D positioning and rotation in the 3D scene object hierarchy are handled by a 3D OpenGL-based library, which greatly simplified our coding. Furthermore, other functions are also supported by this 3D library, such as collision detection, which would be very useful for preventing pipette crash in multielectrode experiments.

\subsection{D structural and functional data mapping}

\subsubsection{Mapping the sample}

At the beginning of an experiment, the first operation is to establish the volumetric contour of the brain slice. This can be done quickly in the 3D scene: under live imaging, the user can simply move the liveview object (and thus the FOV) around the contour of the slice and click with the mouse on the liveview image to sequentially add 3D points to the contour. From these 3D points, a world3d "contour" object will be generated and positioned in the 3D scene. The same procedure can be applied to mark any structural 
feature of interest, or to simply register arbitrary 3D coordinates. All contour objects can be displayed in the 3D scene, and at any time the user can send the liveview to their coordinates, for imaging.

Once the volumetric contour of the slice is constructed, we found it very useful to have a scan of the entire slice surface at low magnification. Together with the volumetric contour, this surface scan provides a reference for navigation with the liveview in the sample space and for further data acquisition, as illustrated in Figs. 3.2, 3.4, and 3.6. The surface scan can be obtained under multiple imaging paradigms. The most obvious choice is IR-DIC or Dodt contrast, which provides images where basic slice anatomy, including neuronal layers, nuclei, etc., can be identified. For transgenic animals with fluorescent labeling, fluorescence scans can be generated as well, and can be shown individually or overlaid onto the IR image. This idea is illustrated in Figs. 3.4 and 3.5, where fluorescently labeled dopaminergic neurons can be seen within midbrain slices. To cover the entire extent of the slice, multiple images were taken and tiled together automatically.

The surface map is a useful prerequisite, but the entire thickness of the slice can potentially be scanned using two-photon imaging or other optically sectioning methods. Ideally, a slice map would have high enough resolution to resolve cellular details and would not take too long to extract. However, two-photon stacks may require significant storage space. For example, acquiring $100 \mathrm{xy}$-planes over a $2 \times 2 \mathrm{~mm}$ sample area with a 40x objective at 512x512 pixel resolution would generate approximately 2 GB of data. Even when storage is sufficient, displaying these image stacks may require more video 
memory than currently available on typical computers. As a simple solution, we have implemented a mechanism where images can be stored at the acquired resolution, but down-sampled and displayed at a lower resolution. Alternatively, the entire map could be displayed at a memory-friendly resolution, but with the current area of interest optionally displayed at maximum resolution.

\subsubsection{Data collection and visualization}

Although constructing a map of the slice could be the final aim in some experiments, in many cases additional data will be obtained. The two types of data generally associated with slice experiments are images and time-resolved optical and electrical signals. For example, electrical signals could be current-clamp or voltage-clamp recordings, while optical signals could be single cell fluorescence using $\mathrm{Ca}^{2+}$ - or voltage-sensitive dyes. The idea is that, throughout the experiment, all images and data are collected and imported on the map with temporal and spatial information, to indicate when and where in the slice they were obtained. An example of how images and time-resolved signals can be embedded on a slice map is shown in Fig. 3.6. A particularly effective way for visualizing $3 \mathrm{D}$ confocal images is to render transparent the pixels where the signal is below a certain threshold, making fluorescently labeled individual neurons stand out against the slice map, as illustrated in the figure.

Our software can be used for acquisition of electrical signals and for image acquisition with camera or laser-scanning sources. Regardless of the image source, acquisition operations can be set up as single images or as scans across one or more spatial dimensions, or across time. For scans, the FOV is programmatically moved by the 
positioning motors to cover either regular geometry space or arbitrary contour objects. Acquisition can be cropped to a smaller region of the sensor, instead of capturing the full frame, to increase the maximum frame rate. Furthermore, time-resolved optical signals corresponding to user-defined regions of interest (ROI) can be extracted in real-time from live images. Recording the average pixel intensity over an ROI, instead of the raw pixel data, dramatically decreases data throughput to only a few bytes per ROI per frame, which is particularly suitable for longer recordings at high frame rates.

These time-resolved optical signals are integrated with the existing functionality in QuB, including the real-time computational engine used for dynamic-clamp (11). For example, this would enable experiments where intracellular calcium concentration could be added to the dynamic-clamp computational model. Specialized world3d objects were implemented in the KPL scripting language to handle images and other types of data, either acquired within our own software or recorded with other programs. These computational objects can be used for script-based image and data processing and for setting up complex data acquisition protocols.

\subsection{Discussion}

We have implemented here some simple but powerful concepts that open new possibilities for experiments in brain slices or other preparations. First, we have identified an easy way to provide structural and functional context to data, in a visually intuitive way. Many programs can be used for offline analysis and interpretation of individual images or other signals. However, what is missing from this approach is the overall picture, where all data can be visually placed together on a 3D map of the sample and 
analyzed. With our software, multiple types of data can be temporally and spatially mapped in living preparations, in real-time. This is a critical step towards understanding brain structure and function at all levels of organization (Getting 1989; Insel et al., 2013).

Second, we have made the experiments themselves easier. There is arguably nothing more frustrating to the neurophysiologist than not knowing where the pipette is located, or not knowing what part of the sample is being imaged by the camera. Is the objective going to crash the pipette into the slice? Is the objective going to crash into the bottom of the recording chamber? This experimental angst has a simple solution. By showing the live image and the recording electrode avatars in a 3D scene, the user is informed at all times where the actual electrodes are and where the live image is coming from. Since this original publication, other systems have been proposed that also function to automate the targeting and patch clamp process (Wu et al., 2016; Suk et al., 2017; Shull et al., 2018; Kolb et al., 2019), as well as new image analysis algorithms for cell identification (Lee and Rozell, 2017) and pipette detection (Koos et al., 2017). 


\subsection{Figures and figure legends}

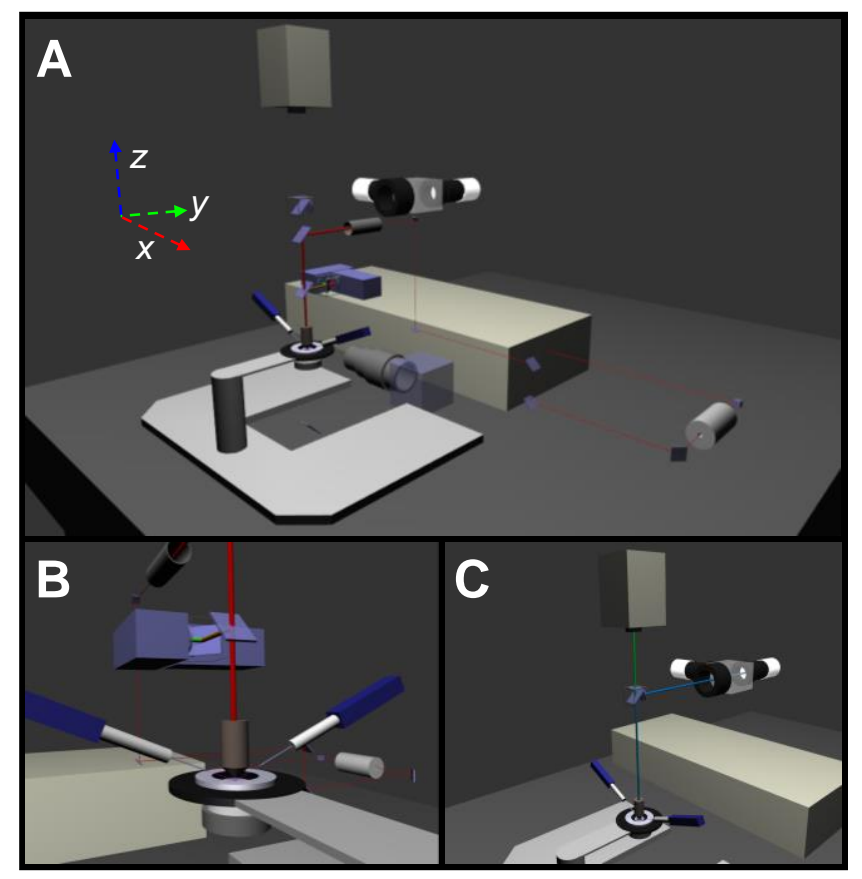

Figure 3.1. Experiment visualization. A 3D scene viewer is used to represent instrumentation, samples, and data. A-C) Computer screenshots showing 3D renderings of an experimental rig equipped for electrophysiology and imaging, functioning in multiphoton (B) or epifluorescence (C) imaging modes. 


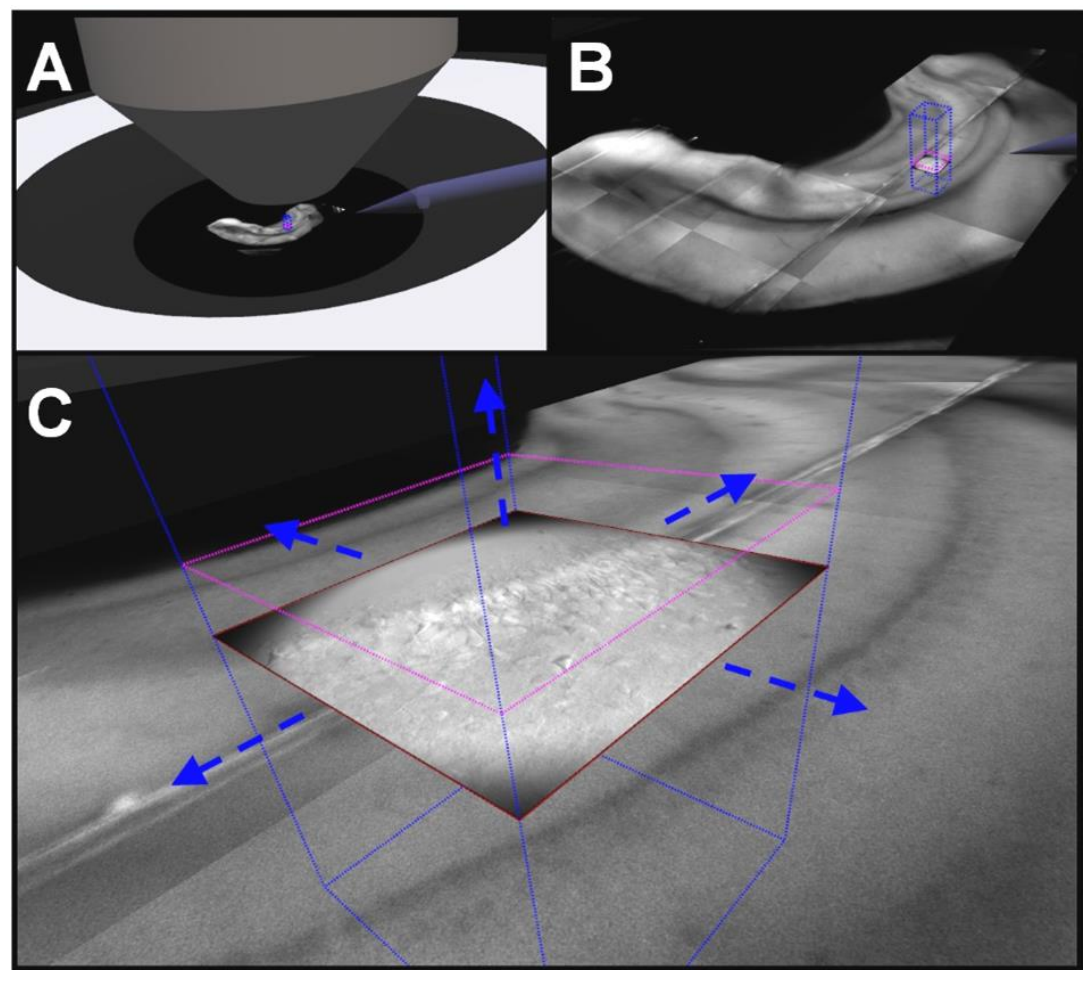

Figure 3.2. Live imaging with real-time position feedback. The live image obtained with the microscopy camera or with the laser scanner is rendered in the 3D scene viewer. A-C) Computer screenshots taken at progressively greater zoom factors, showing the liveview object displaying a live camera image, together with a low-resolution map of the brain slice, the recording chamber, the objective, and a recording electrode. When the FOV is moved in the sample space, the liveview automatically follows, as indicated by the blue arrows, and updates its live content. The blue box denotes the bottom of the chamber and the top of the solution, while the magenta rectangle denotes the top of the slice, which are helpful for successful navigation of the pipette. 


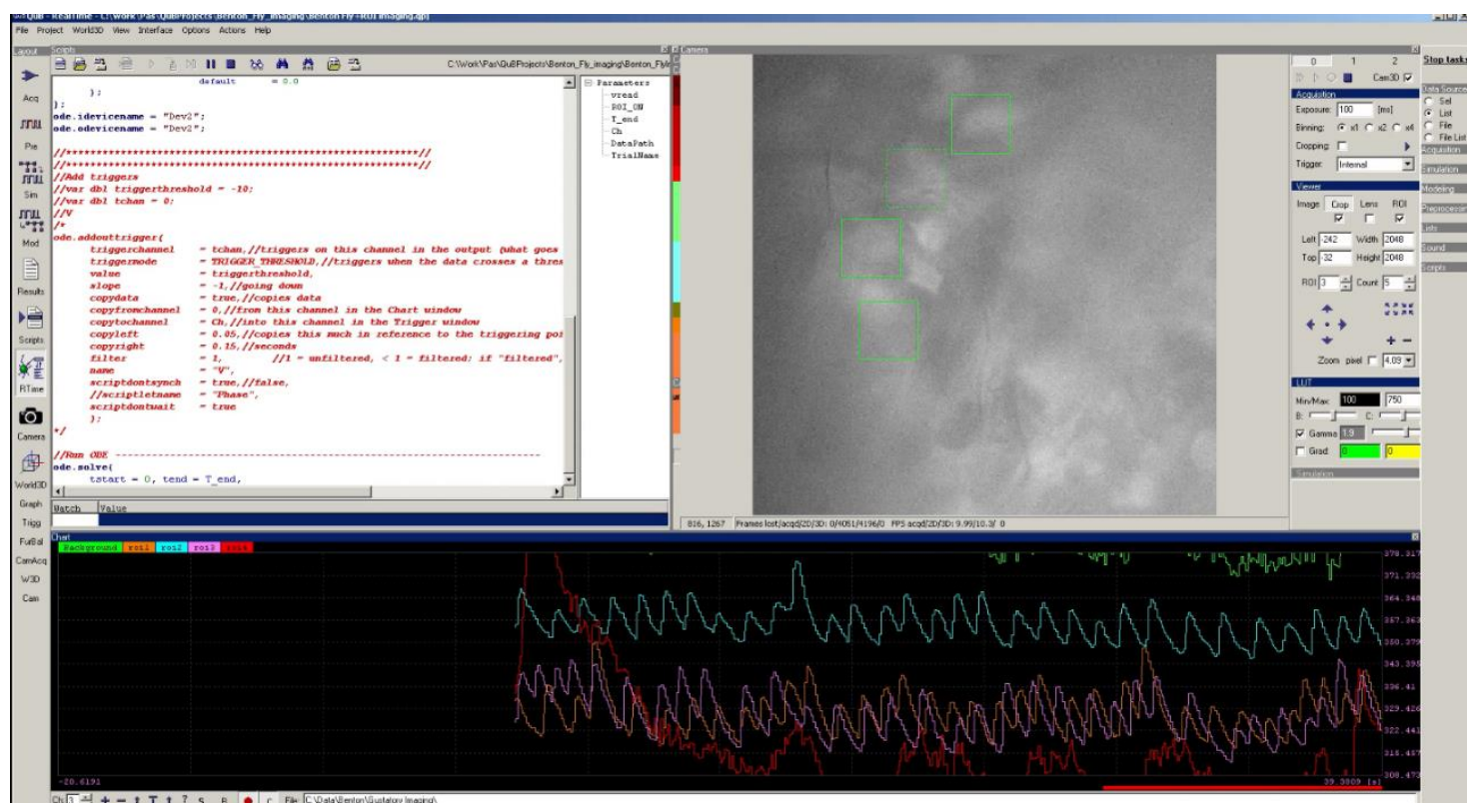

Figure 3.3. Online processing of functional imaging. The screenshot shows the camera window with four active ROIs (green squares) placed over individual cells. The output of the data is plotted in the chart window, where calcium transients corresponding to each individual cell can be measured with relatively high signal to noise ratio. 


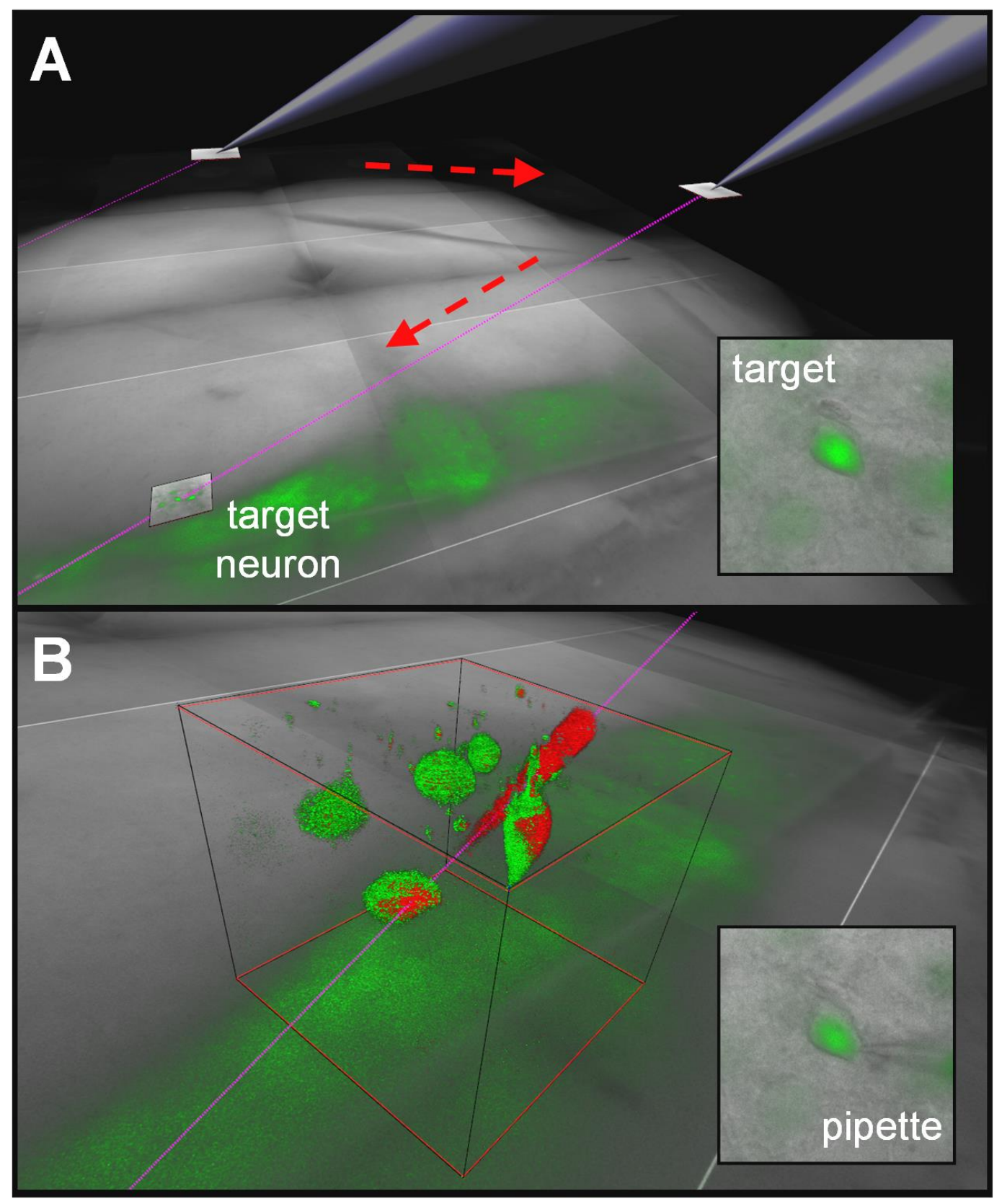

Figure 3.4. Motorized pipette positioning. The pipette is rendered in the $3 \mathrm{D}$ scene viewer, and its position can be updated. Once a target is identified, the pipette can be brought to a position where its approach axis (magenta dotted line) intersects the neuron. A) Computer screenshot illustrating pipette positioning for patching a neuron. $\boldsymbol{B}$ ) Multiphoton z-stack of the slice region around the target neuron, before and after approach. Red color corresponds to the difference between the two z-stacks, as the pipette moved, and two neurons were slightly pushed aside. The insets are IR images taken with the camera before (A) and after $(\mathbf{B})$ target approach. 


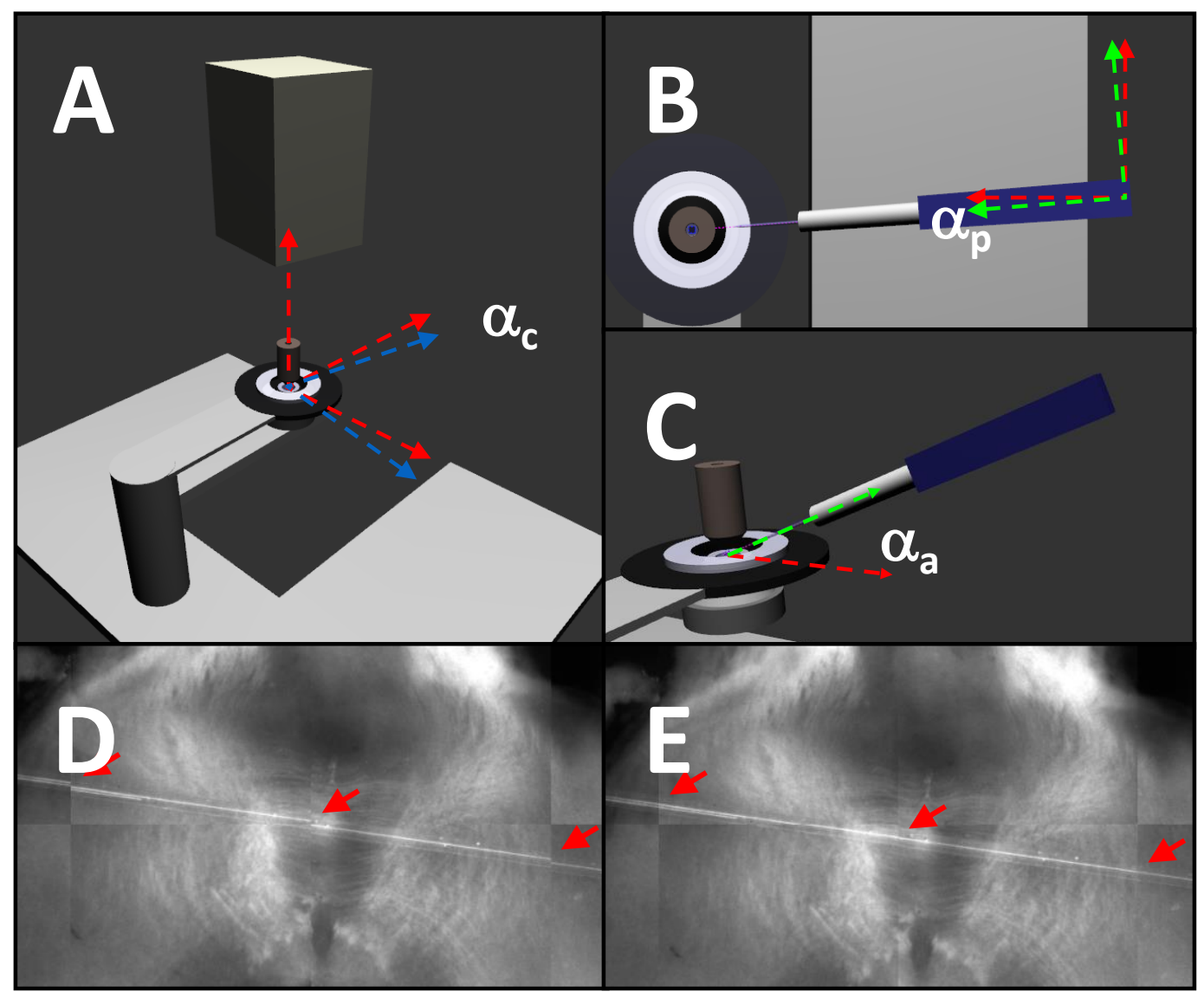

Figure 3.5. Calibrating the motorized positioners. A) Rotational misalignment between the Cartesian axes of the camera sensor (red arrows) and the translation stage (blue arrows). B) Rotational misalignment between the recoding electrode (green arrows) and the camera sensor (red arrows). C) The approach angle of the recording electrode. D) and E) Tiled images before (D) and after (E) implementation of the software correction of the misalignments. 


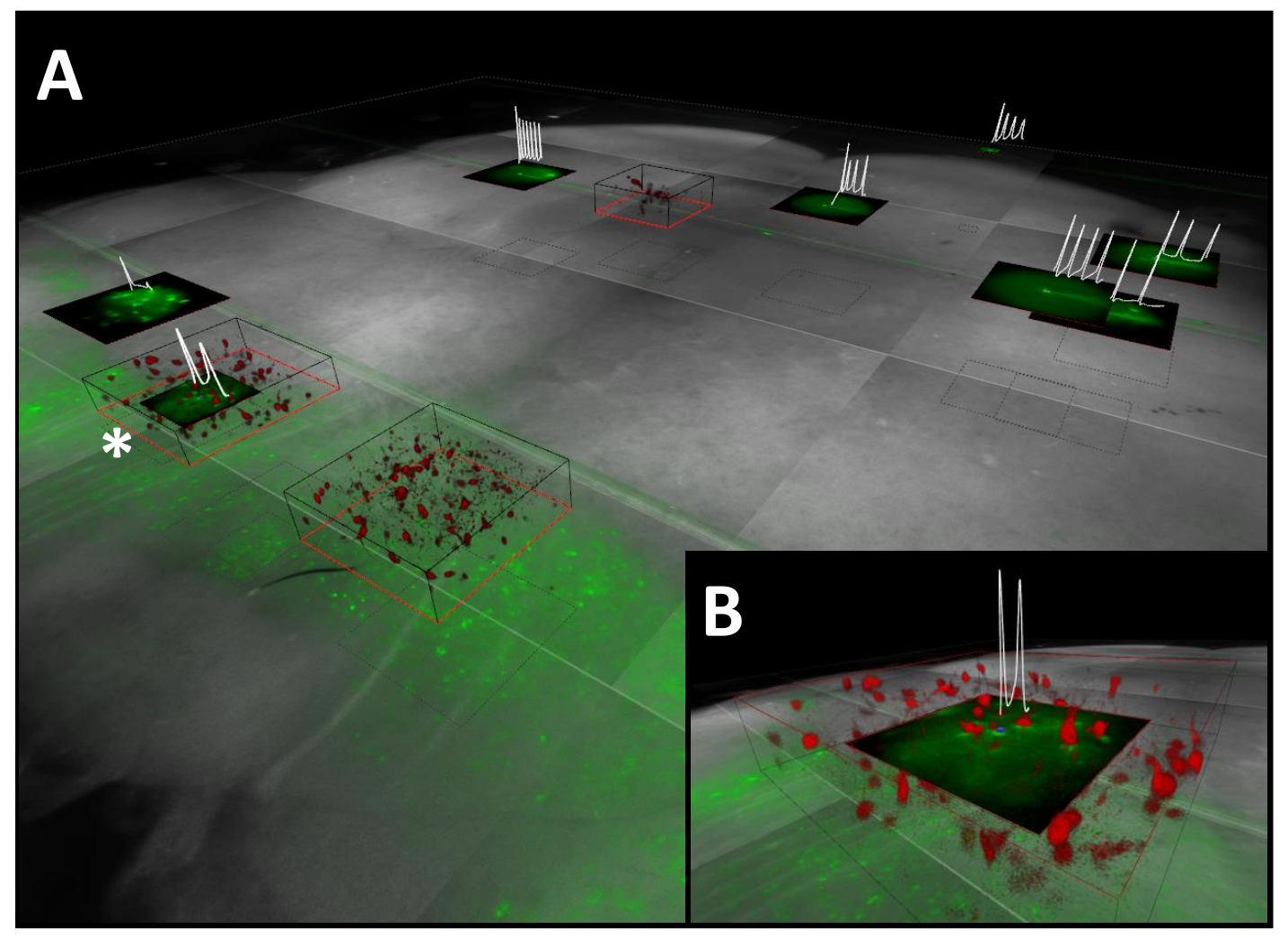

Figure 3.6. 3D data mapping. Different types of data can be rendered in the $3 \mathrm{D}$ scene viewer, including low-resolution transmitted light and epifluorescence maps of the entire brain slice, high-resolution multiphoton z-stacks, and time-resolved electrical and optical signals. Data traces are current clamp recordings from individual cells in response to $100 \mathrm{pA} / 100 \mathrm{~ms}$ pulses. 


\title{
Chapter 4
}

\section{Investigating the role of intracellular calcium on voltage-gated sodium currents}

\author{
This chapter represents unpublished work.
}

\begin{abstract}
Intracellular calcium serves as a signaling factor for many biological processes. Intracellular calcium may also modulate the inactivation kinetics of some Nav channel subtypes. To explore this potential effect, we recorded $\mathrm{Na}^{+}$currents from medullary raphé neurons in neonatal (P0-P3) rat brainstem slices, via whole-cell patch-clamp. Using different calcium buffers, we set the free $\left[\mathrm{Ca}^{2+}\right]$ to low $(\sim 70 \mathrm{nM})$ and high $(\sim 7 \mu \mathrm{M})$ values and characterized Nav channel properties under voltage clamp. We found that high intracellular calcium did not significantly alter steady-state activation kinetics, but slightly hastened inactivation kinetics. The voltage-dependence of inactivation was also rightward shifted $\sim 3 \mathrm{mV}$. Furthermore, we tested whether $\left[\mathrm{Ca}^{2+}\right]$ modifies the kinetics of recovery from inactivation kinetics, using two-pulse protocols applied under both concentrations, and found only minimal effects. Finally, we tested the effect of $\left[\mathrm{Ca}^{2+}\right]$ on Nav use-dependence properties and found a significant reduction in the available current at low $\left[\mathrm{Ca}^{2+}\right]$ compared to high $\left[\mathrm{Ca}^{2+}\right]$. The differences were increased with more depolarizing voltages, indicating that a small shift in the steady-state curves could be the underlying cause.
\end{abstract}


Author contribution: M.A.N designed and performed experiments, analyzed data, created figures, and wrote the manuscript.

\subsection{Background on voltage-gated Na channels}

Voltage-gated $\mathrm{Na}$ (Nav) channels are membrane proteins that open and inactivate quickly (1-2 ms) to depolarize the membrane and elicit an action potential. In humans, there are nine pore-forming $\alpha$-subunits (Nav1.1-1.9), encoded by their respective genes (SCNA1SCNA9) and four single transmembrane (TM) $\beta$-subunits (Nav $\beta 1-4)$, with their respective genes (SCNB1-SCNB4) (Catterall et al., 2005). A single $\alpha$-subunit is often found co-expressed with at least one $\beta$-subunit, and each subunit has differential expression profiles and kinetic modulation (Brackenbury and Isom, 2008). Mammalian Nav $\alpha$-subunits are large (260kDa) complexes organized in four domains (DI-DIV), each with six transmembrane helices (S1-S6) (Fig. 4.1). S1-S4 of each domain acts as the voltage-sensing domain (VSD), whereas S5-S6 of each domain come together to create the conducting pore (Hille, 2001; Ahern et al., 2016).

The high-sequence homology within the voltage-gated ion channel superfamily has greatly accelerated our understanding of Nav channels in terms of the overall pore structure (Lipkind and Fozzard, 2000; Frank et al., 2005), as many crystal structures have been solved for voltage-gated $\mathrm{K}^{+}(\mathrm{Kv})$ channels (Swartz, 2004; Long et al., 2005; González et al., 2012). Pore blocking toxins were used to identify critical residues in the pore (Hidalgo and MacKinnon, 1995), while gating modifier toxins helped establish the S3-S4 segment as the voltage sensing domain (VSD) (Swartz and MacKinnon, 1997a, b). A high density of positively charged residues (Arginine and Lysine), present at every 
third position within the S4 helix defines the binding site of many amphipathic toxins isolated from tarantulas. These toxins "hang" onto the VSD, speeding up activation and slowing deactivation of the channel (Milescu et al., 2007; Mihailescu et al., 2014; Tilley et al., 2018), as discussed in a commentary (Navarro et al., 2019).

\subsubsection{Nav channel function, selectivity, and pharmacology}

Each domain of the $\alpha$-subunit has slightly different sequence in the VSD, which can result in different kinetic properties at the level of individual domains. For Nav channels and many other channels, the movement of DIV is consistently slower than DI-DIII and is tightly correlated with fast inactivation (Stühmer et al., 1989)(Chanda and Bezanilla 2003). By attaching fluorophores onto the cysteines within the S3-S4 linker, many investigators have recorded fluorescent movements that correlate with VSD movement in Kv channels (Manuzzu et al 1996)(Cha and Bezanilla 1997)(Perozo et al 1998)(Glauner et al 1999), Nav channels (Chanda and Bezanilla 2002a; b)(Zhu et al 2017)(Barro-Soria et al 2017) and Cav channels (Pantazis et al 2013). This technique can only be used in heterologous expression systems, which allow for over-amplification of the desired protein and production of relatively large currents that can be conveniently recorded. By averaging data over many repeated trials, the signal to noise can be further improved.

The intracellular linkers between domains serve as regulatory sites of inactivation and other activity. A highly conserved site for Nav channels is the IFM motif along the DIIIDIV linker. When all intracellular linkers are removed with pronase, inactivation is eliminated and $\mathrm{Na}^{+}$currents remain open until the stimulus is over (Armstrong et al., 1973). Addition of the IFM peptide via the patch pipette is enough to rescue inactivation 
(Eaholtz et al., 1994). The IFM motif is conserved across all mammalian Nav channels, as well as fish and bacterial Nav channels (Ahern et al., 2016). Many point mutations have been found in diseases such as cardiomyopathies and epilepsy (Deschênes et al., 1999).

Nav channels are highly selective for $\mathrm{Na}^{+}$, but other monovalent cations, such as $\mathrm{K}^{+}$and $\mathrm{Li}^{+}$, can also pass, albeit with lower affinity. Selectivity or permeability is on the order of 170:1 for $\mathrm{Na}^{+}: \mathrm{K}^{+}$and 70:1 for $\mathrm{Na}^{+}: \mathrm{Ca}^{2+}$ (Hille, 2001; Ren et al., 2001b). Mutations in critical charged residues in the S5-S6 linker were used to identify the selectivity filter and the outer vestibule, as neutralization of negatively charged amino acids greatly reduces tetrodotoxin (TTX) sensitivity (Fozzard and Hanck, 1996). TTX is a highly-selective and potent $\left(\mathrm{IC}_{50} \sim 60 \mathrm{nM}\right)$ pore blocker of Nav channels that was originally isolated from pufferfish, where it is produced by symbiotic bacteria (Do et al., 1990; Yu et al., 2004), which has also been found on terrestrial amphibians (Cardall et al., 2004; Hanifin, 2010). The high specificity of TTX for Nav channels (Nav1.1-Nav1.6) has been a priceless tool for understanding not only ion channel structure, but also neural behavior.

\subsubsection{Nav channel subtype specification and expression}

The primary subtypes in the mammalian central nervous system are Nav1.1, Nav1.2 Nav1.3 and Nav1.6, whereas Nav1.7, Nav1.8, and Nav1.9 are largely expressed in the peripheral nervous system (Elliott and Elliott, 1993; Strickland et al., 2008). Nav1.2, Nav1.3, and Nav1.6 are found in t-tubules of skeletal muscle, while Nav1.4 is typically located on the intercalated disk (Maier et al., 2002; Wang and Wang, 2003; Hirn et al., 2008). Nav1.5 is known as the cardiac muscle subtype (Remme et al., 2008) and many 
mutations lead to diseases such as tachycardia, heart failure, long-qt syndrome, and Brugada syndrome (Papadatos et al., 2002; Mohler et al., 2004; Nattel et al., 2007). Nav1.3 is also responsible for pacemaking currents found in serotonin-releasing enterochromaffin cells in the gut (Strege et al., 2003; Strege et al., 2017). In neurons, Nav channels are distributed throughout the cell, in the soma, dendrites, and axon (Lai and Jan, 2006; Vacher et al., 2008). Nav channels are also found at high densities in the axon initial segment (AIS) (Palay et al., 1968; Kole, 2008; Lorincz and Nusser, 2010), a highly organized structure within the first 20-60 $\mu \mathrm{m}$ of axon, present in almost all vertebrate neurons (Chang and Rasband, 2013).The AIS is composed of dense actin and $\beta 4$-spectrin cytoskeleton, with an underlying macromolecule called AnkyrinG (AnkG), which serves as a landing zone for voltage-gated ion channels, neurotransmitter receptors, and cell adhesion molecules (Rasband, 2010; Gasser et al., 2012).

Nav channel expression within the AIS is also specific, with Nav1.6 often found distally, further away from the soma, and Nav1.1 or Nav1.2 proximal to the soma (Hu et al., 2009; Osorio et al., 2010; Yamagata et al., 2017). As Nav1.6 has a slightly ( 5 to $10 \mathrm{mV})$ more hyperpolarized activation curve, these dense and isolated channels can lower the local resting membrane potential, making it easier for Nav channels to activate (Yu et al., 2008; Hu et al., 2009; Teleńczuk et al., 2016). For these reasons, the AIS is the site of action potential (AP) initiation (Colbert and Johnston, 1996; Kole, 2008; Hu et al., 2009; Baranauskas et al., 2013). From the axonal initial segment, the action potential backpropagates to the soma, causing the abrupt onset (Stuart et al., 1997b; Palmer and Stuart, 2006; Shu et al., 2007; Yu et al., 2008). A compromised structural integrity of the AIS is 
a factor in many complex neurological disorders (Buffington and Rasband, 2011), including Angelmann syndrome (Kaphzan et al., 2011; Kaphzan et al., 2013), schizophrenia (Volk et al., 2002; Athanasiu et al., 2010), and bipolar disorder (Schulze et al., 2009).

\subsubsection{Modulators of Nav channel kinetics}

Nav channels have many intrinsic sites for regulation by a variety of intracellular factors. There are many Serine/Threonine residues that can be phosphorylated by PKA and PKC (Costa and Catterall, 1984; West et al., 1991; Chen et al., 1995; McAnelly and Zakon, 1996; Cantrell et al., 2002), which in turn can up-regulate or down-regulate protein expression and modify overall current density (Misonou et al., 2004; Misonou and Trimmer, 2004). In heterologous systems, the co-expression of Nav $\beta 1$ subunit along with any $\alpha$-subunit greatly enhances overall expression (Chahine and O'Leary, 2011) as well as shifting the steady-state activation and inactivation curves. Expression of the Nav $\beta 4$ subunit, or even of a small portion of the intracellular tail (Aman and Raman, 2010; Lewis and Raman, 2013), results in an open-pore block by $\beta 4$, which competes with the fast-inactivation particle IFM motif and generates a "resurgent" current (Raman and Bean, 1997) when the particle is released (Bant and Raman, 2010). This behavior is a key mechanism that allows for the fast firing rates $(\sim 50 \mathrm{~Hz})$ of cerebellar Purkinje neurons. However, these regulatory mechanisms change with cellular activity, making it challenging to separate them from other cellular functions.

The C-terminal tail of voltage-gated channels is often a site for modulation. Nav channel C-terminus includes a calmodulin binding motif and an IQ domain, which allows for 
modulation by intracellular calcium. Calmodulin is a calcium binding protein composed of two EF-hand domains, which bind two $\mathrm{Ca}^{2+}$ ions per domain (Chin and Means, 2000). Binding of $\mathrm{Ca}^{2+}$ to calmodulin allows for transportation of $\mathrm{Ca}^{2+}$ ions that upon activation can trigger many downstream events such as regulation of gene expression by activation of CREB (cAMP response element binding protein)(Kandel, 2012) or CamKII (calmodulin kinase kinase), which are required for long-term potentiation (Herring and Nicoll, 2016; Nakahata and Yasuda, 2018).

Recently, it was found that the AIS macrostructure has activity-dependent plasticity, expressed via shortening and relocating the expression of AIS structural proteins (Grubb and Burrone, 2010). During development, the AIS undergoes both elongation and shortening, regulated by overall neuronal activity on the timescale of days to weeks (Grubb et al., 2011; Kuba, 2012; Hinman et al., 2013; Kuba et al., 2014; Yoshimura and Rasband, 2014), or even hours in culture (Grubb et al., 2011; Evans et al., 2013; Evans et al., 2015). It is thought that AIS relocation is triggered by an increase in intracellular calcium levels, via activation of L-type calcium channels and of a downstream calciumsensitive phosphatase, calcineurin (Grubb and Burrone, 2010; Bender et al., 2012). Inhibition of the calcineurin can prevent AIS relocation (Evans et al., 2013).

\subsection{Chapter introduction}

The regulation of ion concentrations within cellular compartments is critical for cellular communication. Cellular gradients of permeant ions regulate many important cellular functions, such as ATP production (Elston et al., 1998), water regulation (Hazama and Okada, 1988), and gene expression (Bading et al., 1993; Dolmetsch et al., 1998). In 
addition to its electrogenic function, $\mathrm{Ca}^{2+}$ ions can participate in signal cascades that trigger synaptic release (Katz and Miledi, 1968), cross-bridge cycling of the actin-myosin filaments (Metzger and Moss, 1990), and many forms of synaptic plasticity via $\mathrm{Ca}^{2+}$ activated phosphatases (calcineurin) and kinases (calmodulin-dependent kinase-2) (Malinow et al., 1989; Malenka, 1994; Evans et al., 2013). Many calcium activated proteins have structural domains known as $\mathrm{EF}$ hand motifs, which can bind two $\mathrm{Ca}^{2+}$ ions each (Persechini et al., 1989). Calmodulin (CaM), an intracellular calcium binding protein is made up of two lobes, each composed of two EF hands that allow the binding of $\mathrm{Ca}^{2+}$ with high affinity $(\mathrm{nM}-\mu \mathrm{M})$ (Faas et al., 2011). As a mobile, intracellular protein, $\mathrm{CaM}$ activity serves as a local sensor of $\mathrm{Ca}^{2+}$ activity to regulate many members of the ion channel superfamily, including voltage-gated $\mathrm{Ca}^{2+}(\mathrm{Cav})$ channels (Peterson et al., 1999), store-operated Ca-release channels ( $\mathrm{Li}$ et al., 2017), Ca-activated $\mathrm{K}^{+}$channels (Keen et al., 1999; Joiner et al., 2001), Ca-activated Cl channels (Tian et al., 2011; Jung et al., 2013), voltage-gated K (Kv) channels (Wen and Levitan, 2002; Chang et al., 2018), voltage-gated $\mathrm{Na}$ (Nav) channels (Deschênes et al., 2002; Ben-Johny et al., 2014) and Transient Receptor potential (TRP) channels (Lambers et al., 2004; Hasan et al., 2017). Binding of CaM to functional proteins often occurs at sites known as IQ (IsoleucineGlutamine) domains. These domains are found in many $\mathrm{Ca}^{2+}$-regulated proteins (Yamniuk and Vogel, 2004); in voltage-gated Cav and Nav channels, they are found on the C-terminal tail (Wen and Levitan, 2002; Herzog et al., 2003; Ben-Johny et al., 2015). For Cav channels, non-Ca ${ }^{2+}$-bound Calmodulin (apo-Calmodulin) binds to the $\mathrm{C}$ terminus and is important for regulating the $\mathrm{Ca}^{2+}$-dependent inactivation, which is 
isolated using $\mathrm{Ba}^{2+}$ as a charge carrier (Tang et al., 2003; Adams et al., 2014).

Functionally, calcium dependent inactivation (CDI) serves as a negative - feedback mechanism to reduce channel activity once appropriate intracellular $\mathrm{Ca}^{2+}$ levels are reached. Intracellular $\mathrm{Ca}^{2+}$ concentrations $\left(\left[\mathrm{Ca}^{2+}\right]\right)$ are tightly regulated in cells, as high $\left[\mathrm{Ca}^{2+}\right]($ e.g., $>10-15 \mu \mathrm{M})$ can activate calpains, which are $\mathrm{Ca}^{2+}$-activated proteases that degrade structural proteins within the cell at the beginning stages of apoptosis and trigger cell death (Squier et al., 1994).

In resting neurons, intracellular $\left[\mathrm{Ca}^{2+}\right]$ remains around $100 \mathrm{nM}$ (Helmchen et al., 1996) and can increase to a few $\mu \mathrm{M}$ during high firing activity. During an AP, the membrane depolarization activates Cav channels in the axon terminus, triggering an increase in $\left[\mathrm{Ca}^{2+}\right](>1 \mu \mathrm{M})($ Lipscombe et al., 1988), inducing neurotransmitter release. Upon ligand binding, many ionic neurotransmitter receptors, such as NMDA glutamate receptors and TRP channels, permeate a non-selective current dominated by $\mathrm{Ca}^{2+}$ (MacDermott et al., 1986), leading to increase in $\left[\mathrm{Ca}^{2+}\right]$ within dendritic spines (Yuste and Denk, 1995;

Sabatini et al., 2002) and throughout the cell. The use of fluorescently labelled calcium chelators that modify their brightness when $\mathrm{Ca}^{2+}$ is bound (Kao et al., 1989; Regehr et al., 1989; Maravall et al., 2000) and genetically engineered probes (Miyawaki et al., 1997; Nakai et al., 2001; Akerboom et al., 2012; Chen et al., 2013) has been instrumental for unveiling how $\mathrm{Ca}^{2+}$ activity increases proportional to neuronal firing frequency (Helmchen et al., 1996; Magee and Johnston, 1997; Markram et al., 1997).

As neurons require voltage-gated Nav channels to fire APs, modulation of Nav channel kinetics can regulate overall neuronal activity. Nav channels begin to open at 
depolarizing potentials (around $-50 \mathrm{mV}$ ), further driving membrane depolarization and thus opening more Nav channels. Once opened, Nav channels quickly inactivate (2-5 ms) and cease conduction, allowing for repolarization of the membrane by Kv currents and for reliable conduction of the AP down the axon. However, not all Nav channels inactivate with the same kinetics. In some neurons, Nav channels exhibit partial inactivation, or a "persistent" component (French et al., 1990; Crill, 1996), whereas in other neurons, Nav channels exhibit long-term inactivation that lasts hundreds of milliseconds or seconds (Colbert et al., 1997; Jung et al., 1997; Mickus et al., 1999; Aman and Raman, 2007; Milescu et al., 2010b). Some Nav channels also exhibit calcium dependent inactivation (CDI), such as Nav1.4, found in skeletal muscle (Herzog et al., 2003; Biswas et al., 2009; Ben-Johny et al., 2014), and Nav1.2, found in neurons (Chahine et al., 2005; Wang et al., 2012).

The ventral raphé are a group of serotonergic pacemaking neurons located in the midline of the brainstem, providing serotonin (5-hydroxytryptophan, 5-HT) to the brainstem and spinal cord circuits (Richerson, 2004). When serotonergic neurons cannot produce 5HT or are not present, animals have severe respiratory problems and often die in infancy (Hodges et al., 2009). Sitting at the seam of the brainstem, the raphé neurons wrap processes around blood vessels and increase their firing rate (from $2 \mathrm{~Hz}$ to $6 \mathrm{~Hz}$ ) when perfused with high $\mathrm{CO}_{2}$ (Wang et al., 1998; Wang et al., 2002; Severson, 2003; Corcoran et al., 2009). By increasing their firing rate, they increase the release of 5HT onto the rhythmic inspiratory pacemakers, the pre-Bötzinger complex (pre-BötC), which triggers an increase in respiratory rhythm (Wang et al., 1998; Wang et al., 2001; Severson, 2003; 
Ptak et al., 2009). These neurons are implicated in disease states such as sudden infant death syndrome and congenital sleep apnea (Richerson, 1997; Richerson, 2004).

The Nav currents in the ventral raphé exhibit long-term inactivation (LTI), where a single AP or voltage clamp pulse can trigger a proportion of channels $(20 \%)$ to become activated for seconds (Milescu et al., 2010b). Long-term inactivation of Nav channels has been attributed to proteins known as Fibroblast-Growth Factor Homologous Factors (FHF1-4 or FGF11-14), which contain the immunoglobulin domain characteristic to members of the fibroblast growth factor family. However, they do not bind to the FGF receptor, are not secreted, and do not serve as growth factors (Smallwood et al., 1996; Ornitz, 2000; Wang et al., 2000; Goldfarb, 2005). FHFs are intracellular proteins that coexpress with Nav channels (Smallwood et al., 1996; Liu et al., 2001; Wittmack et al., 2004; Goetz et al., 2009) and can regulate Nav peak amplitude and dramatically slow inactivation rates (Liu et al., 2003; Goldfarb et al., 2007; Laezza et al., 2007; Laezza et al., 2009; Dover et al., 2010; Goldfarb, 2012; Venkatesan et al., 2014). Crystal structures of the C-terminus of Nav 1.5 and Nav1.2 channels have been crystallized in a complex with FGF13 and calmodulin, in a calcium-dependent manner (Goetz et al., 2009; Wang et al., 2011b; Sarhan et al., 2012; Wang et al., 2012). FHF and Calmodulin bind to the Cterminus at different levels of $\left[\mathrm{Ca}^{2+}\right]$ indicates that these regulators of Nav channel inactivation may be kinetically interacting. In this chapter, I present a series of patchclamp electrophysiology experiments in ventral raphé neurons, where we tested if intracellular $\left[\mathrm{Ca}^{2+}\right]$ can modulate Nav channel kinetics, particularly long-term inactivation. 


\subsection{Materials and methods}

\section{$\underline{4.3 .1 \text { Animal procedures }}$}

All animal procedures were approved by the University of Missouri Animal Care and Use Committee. Neonatal (P1-P4) Sprague Dawley rats (Taconic, USA) were used following standard slice preparation and electrophysiology procedures.

\section{$\underline{4.3 .2 \text { Brain slice preparation }}$}

In vitro medullary slice preparations containing midline raphé neurons, inferior olivary neurons, and hypoglossal motor neurons were obtained from postnatal (P0-P5) Sprague Dawley rats as described in (Koshiya and Smith, 1999). Briefly, animals were anesthetized with isoflurane and the brain was removed from the skull in ice-cold artificial CSF (aCSF) containing (in $\mathrm{mM}$ ): $124 \mathrm{NaCl}, 25 \mathrm{NaHCO}_{3}, 3 \mathrm{KCl}, 1.5 \mathrm{CaCl}_{2}, 1.0$ $\mathrm{MgSO}_{4}, 0.5 \mathrm{NaH}_{2} \mathrm{PO}_{4}$, and $30 \mathrm{D}$-glucose, equilibrated with $95 \% \mathrm{O}_{2}$ and $5 \% \mathrm{CO}_{2}(\mathrm{pH} 7.4$ \pm 0.05 at room temperature). Fine dissection was quickly performed to remove the pia membrane to increase oxygen penetration to the tissue. The brainstem was pinned on to a piece of clay attached to an acrylic mount, which was attached on top of a metal base that could be manipulated with a set screw, to properly orient the brainstem for slicing. Transverse brainstem slices were cut rostral to caudal in $400 \mu \mathrm{m}$ thick sections, until the opening of the fourth ventricle was seen. The wall of the fourth ventricle was used to adjust the pitch of the mounting device. $200 \mu \mathrm{m}$ thick sections were taken until the desired location was determined, indicated by the presence of the inferior olives, the first hypoglossal (XII) nerve rootlet, and the presence of the nucleus ambiguous. Once in final 
position, a 350-400 $\mu \mathrm{m}$ thick slice was taken at the level of the pre-BotC. The slice was transferred to a recording chamber mounted onto a microscope stage, and superfused with aCSF at room temperature.

\subsubsection{Neuron identification}

Ventral raphé neurons were selected based on their location in the slice and their electrical activity. Raphé neurons cluster at the midline immediately superior to the inferior olives and have irregularly shaped somas. After obtaining a G $\Omega$ seal and prior to breaking into whole-cell configuration, the amplifier was switched to current clamp (CC) mode to check for regular and slow pacemaking $(1-3 \mathrm{~Hz})$ with broad action potentials (3-6 ms). All experiments were done under visual control using infrared-Dodt contrast for visualization.

\section{$\underline{\text { 4.3.4 Solutions }}$}

Intracellular solutions were designed to obtain a desired free $\left[\mathrm{Ca}^{2+}\right]$ using the MaxChelator program (Chris Patton) to calculate buffering capacity. For voltage clamp (VC), pipettes were filled with a "Low Calcium" solution with a calculated free $\left[\mathrm{Ca}^{2+}\right] \approx$ $70 \mathrm{nM}$, containing (in mM): 120 Cs-gluconate (prepared from $\mathrm{CsOH}$ and gluconic acid), 30 Na-gluconate, 10 TEA-Cl, 5 4-AP, 10 EGTA, $1 \mathrm{CaCl}_{2}$, 10 HEPES, 4 Mg-ATP, 0.3 $\mathrm{Na}_{3}$-GTP, $10 \mathrm{Na}_{2}$-phosphocreatine, pH 7.3 adjusted with $\mathrm{CsOH}(285 \pm 5 \mathrm{mOsm} / \mathrm{L})$. To create the "High Calcium" solution with a calculated free $\left[\mathrm{Ca}^{2+}\right] \approx 7 \mu \mathrm{M}$, HEDTA was used as the $\mathrm{Ca}^{2+}$ chelator, as it can set free $\left[\mathrm{Ca}^{2+}\right]$ to $\mu \mathrm{M}$ levels. This solution contained the following (in $\mathrm{mM}$ ): $125 \mathrm{Cs}$-gluconate (prepared from $\mathrm{CsOH}$ and gluconic acid), 10 
TEA-Cl, 5 4-AP, $10 \mathrm{Na}_{3}$-HEDTA, $5 \mathrm{CaCl}_{2}$, 10 HEPES, $4 \mathrm{Mg}$-ATP, $0.3 \mathrm{Na}_{3}$-GTP, 10 $\mathrm{Na}_{2}$-phosphocreatine, $\mathrm{pH} 7.3$ adjusted with $\mathrm{CsOH}(285 \pm 5 \mathrm{mOsm} / \mathrm{L})$. The intracellular $\left[\mathrm{Na}^{+}\right]$for both $\mathrm{VC}$ solutions was set to $50 \mathrm{mM}$ to reduce the size of $\mathrm{Na}^{+}$currents and improve voltage- and space-clamp.

For CC, pipettes were filled with a "Low Calcium" solution containing (in mM): $125 \mathrm{~K}$ gluconate, $10 \mathrm{NaCl}, 10$ EGTA, $1 \mathrm{CaCl}_{2}, 10$ HEPES, 4 Mg-ATP, $0.3 \mathrm{Na}_{3}$-GTP, $4 \mathrm{Na}_{2}-$ phosphocreatine, pH 7.4 adjusted with $\mathrm{KOH}(285 \pm 5 \mathrm{mOsm} / \mathrm{L})$. For the "High Calcium" CC experiments, the solution contained (in mM): $125 \mathrm{~K}$-gluconate, $10 \mathrm{Na}_{3}$-HEDTA, 5 $\mathrm{CaCl}_{2}, 10$ HEPES, 4 Mg-ATP, 0.3 Na3-GTP, 4 Na2-phosphocreatine, $\mathrm{pH} 7.4$ adjusted with $\mathrm{KOH}(285 \pm 5 \mathrm{mOsm} / \mathrm{L})$. A fluorescent dye (TxRed, 10 $\mu \mathrm{M})$ was added to the pipette solution prior to filtering, to highlight neurons accessed by whole-cell.

\subsubsection{Pharmacology}

For VC experiments, $\mathrm{CdCl}_{2}(200 \mu \mathrm{M})$ was added to the superfusing aCSF to block $\mathrm{Ca}^{2+}$ currents. In some slice experiments, 1,2,3,4-tetrahydro-6-nitro-2,3-dioxobenzo[ $f]$ quinoxaline-7-sulfonamide (NBQX; $10 \mu \mathrm{M}$ ) was added to the superfusing aCSF to inhibit excitatory synaptic transmission. To block $\mathrm{Na}^{+}$currents, TTX $(1 \mu \mathrm{M})$ was added to the superfusing aCSF after initial $\mathrm{VC}$ recordings were taken. All reagents were purchased from Millipore-Sigma (St. Louis, MO).

\subsubsection{Electrophysiology}

Electrodes (4-5 M 2 ) were pulled from borosilicate glass, fire polished on a micro-forge and then coated with a thin layer of Sylgard to reduce capacitive transients. Pipette 
capacitance was compensated $100 \%$ in $\mathrm{VC}$, and $\approx 70 \%$ in CC. For off-line analysis, the membrane capacitance $\left(C_{\mathrm{m}}\right)$ was approximated as the value used for compensation in VC $(\approx 20 \mathrm{pF})$. Series resistance $\left(R_{\mathrm{s}}\right)$ was typically 9-15 $\mathrm{M} \Omega$. Cells with $R_{\mathrm{s}}>22 \mathrm{M} \Omega$ were discarded. In VC experiments, $R_{\mathrm{s}}$ was compensated $80 \%$ ( $2 \mu$ s response time), and the compensation was readjusted before each voltage clamp protocol. For CC experiments, bridge mode was compensated $100 \%$ and periodically readjusted. A calculated liquid junction potential of $\approx 10 \mathrm{mV}$ for the $\mathrm{K}^{+}$-based and $\approx 8 \mathrm{mV}$ for the $\mathrm{Cs}^{+}$-based solutions was corrected on-line. Somatic whole-cell recordings were obtained with a HEKA EPC10 Double patch clamp amplifier. For VC, data were low-pass filtered at $40 \mathrm{kHz}$ and digitally sampled at $100 \mathrm{kHz}$, using the amplifier's built-in digitizer, controlled by Patchmaster software from HEKA (v2.91). For analysis, all VC data were then downsampled to $10 \mathrm{kHz}$ and filtered at $5 \mathrm{kHz}$ to reduce file size. For $\mathrm{CC}$, the signal representing membrane voltage was digitally sampled at $50 \mathrm{kHz}$ (open-bandwidth), using National Instruments data acquisition cards (PCI 6052E or PCI 6251) and NI-DAQmx 8.1, controlled by the QuB software (http://milesculabs.biology.missouri.edu/QuB.html).

\subsubsection{Voltage clamp protocols}

VC protocols were constructed and applied with the Patchmaster program. Leak subtraction was performed online with the $n / 4$ method. To ensure stability between sweeps, the inter-sweep interval for all voltage protocols was set to 6 seconds, holding at $-80 \mathrm{mV}$, to give sufficient time to Nav channels to exit the LTI state. For protocols investigating the recovery after use dependence, an inter-sweep interval of 30 seconds was used. Experimental VC protocols were applied 5 mins after establishing the whole- 
cell configuration. To test stationarity between sweeps, a brief $1 \mathrm{~ms}$ pulse to $-15 \mathrm{mV}$ was applied at least $25 \mathrm{~ms}$ prior to each protocol. If the current elicited by this pulse changed by $>12 \%$ from the first to the last sweep, the protocol was eliminated from the dataset.

To assess steady-state (SS) activation (SSAct), a voltage pre-pulse of (-40 mV, 4-5 ms) was utilized prior to the test step, to inactivate axonal Nav channels for better space clamp (Milescu et al., 2010a). Activation voltage was measured from -100 to $+20 \mathrm{mV}$, in increments of $+5 \mathrm{mV}$, followed by a step to $-20 \mathrm{mV}$ for $100 \mathrm{~ms}$, followed by a repolarization to $-120 \mathrm{mV}$. The maximum amplitude of the current was taken during the first 50ms of the test step and baseline subtracted. IV relationships were plotted in QuB and converted to GV curves as calculated by Imax/(Vstep-Vrev), when Vrev $=33 \mathrm{mV}$ is the voltage when the $\mathrm{Na}^{+}$current reversed, as measured in voltage clamp and confirmed by the GHK equation. Curves were then fit with a Boltzmann function multiplied by a line $(\mathrm{Y}=$ Bottom $+($ Top-Bottom + Slope $1 * \mathrm{X}) /(1+\exp ((\mathrm{V} 50-\mathrm{X}) /$ Slope $))$ in Prism4.1 (Graphpad).

To measure SS-inactivation (SS-InAct), multiple protocols were used. First, the $-20 \mathrm{mV}$ holding step after the SS-Act protocol allows one to calculate both SSAct and SSInAct from the same protocol, as the test step for Activation is simply the pre-pulse for Inactivation. To disambiguate any effect of the $\mathrm{VC}$ prepulse, separate protocols were created without a prepulse, where a conditioning-pulse from -100 to $-20 \mathrm{mV}$ in $+5 \mathrm{mV}$ increments was applied for 50, 100 and $300 \mathrm{~ms}$, followed by the test step at $-20 \mathrm{mV}$ for $100 \mathrm{~ms}$. The peak of the activation IV curve is typically between -15 and $-20 \mathrm{mV}$. Data were analyzed in QuB by normalizing the peak current of each test pulse (taken at - 
$20 \mathrm{mV}$ ) to the maximum current. IV curves were fit with a Boltzmann function ( $\mathrm{Y}=$ Bottom + (Top-Bottom + Slope1) / (1+ exp $((\mathrm{V} 50-\mathrm{X}) /$ Slope $))$ in Prism4.1

Recovery from inactivation (RI) was determined with a two-pulse protocol. After the minimum 6 second inter-sweep interval holding at $-80 \mathrm{mV}$, a test pulse to $-15 \mathrm{mV}$ for 5 ms was applied, followed by a variable holding step at $-80 \mathrm{mV}$ or $-65 \mathrm{mV}$ (starting at $0.75 \mathrm{~ms}$ and multiplied by 1.8 for each sweep), followed by another $5 \mathrm{~ms},-15 \mathrm{mV}$ test pulse, repeated for 17 sweeps. This protocol results in exponentially increasing intervals that efficiently sample the exponential recovery time course. Although in heterologous systems the recovery protocols typically have holding voltages below $-100 \mathrm{mV}$, raphé neurons cannot be reliably held at such negative potentials for too long without destroying the patch. The $-65 \mathrm{mV}$ holding voltage was used as it is close to the typical resting potential (Milescu et al., 2010b). We tested voltages above $-65 \mathrm{mV}$, but many neurons could not be reliably clamped for the entire length of the recovery protocol. Analysis was done by taking the ratio between the maximum current evoked by the second pulse relative to the first pulse, for each sweep. Bi-exponential functions were fit to each dataset using the following equation $\mathrm{Y}=\mathrm{Amp} 1 * \exp (-\mathrm{X} / \mathrm{tau} 1)+\mathrm{Amp} 2 * \exp (-$ $\mathrm{X} /$ tau2) + IEq. Multiple rounds of fitting were performed with various constraints to compare the data sets.

Use-dependent inactivation was measured with repetitive voltage pulses applied at different frequencies. As a first approximation of use-dependence, four pulses from -80 to $-15 \mathrm{mV}$ were separated by either $40 \mathrm{~ms}, 100 \mathrm{~ms}$, or $300 \mathrm{~ms}$. The four pulses were applied three times and the ratios were averaged across the three sweeps. Data were analyzed by 
taking the ratio of the maximum current evoked by a given pulse relative to the first pulse. A 20-pulse protocol with repetition frequencies of $2 \mathrm{~Hz}, 5 \mathrm{~Hz}$, and $12 \mathrm{~Hz}$ was applied and processed in the same way. To test the extent to which these neurons can still generate $\mathrm{Na}^{+}$currents, we also applied a few protocols consisting of 100 pulses repeated at $5 \mathrm{~Hz}, 10 \mathrm{~Hz}, 20 \mathrm{~Hz}$, and $50 \mathrm{~Hz}$. The data with large variations in the current during the holding voltage, signifying poor VC, were discarded.

\subsubsection{Data analysis and statistics}

Custom-made scripts were developed in the QuB software (http://milesculabs.biology.missouri.edu/QuB.html) to process VC and CC data. Scripts were also generated to implement ion channel fitting, to test ion channels in computational neuron models, and to run simulations. Statistics and curve fitting were performed with Prism 4.1 (Graphpad) software.

\subsubsection{Data acquisition hardware}

Experiments were performed on a customized upright microscope (Scientifica, UK) equipped with Dodt contrast, epifluorescence, and two-photon imaging with a MaiTai HP laser (Newport, USA), and with computer-controlled motorized positioners (Scientifica, UK) for the translation stage, objective, and recording electrodes. Wide-field imaging was done with a Hamamatsu sCMOS Flash4.0 V2 camera (Hamamatsu Photonics KK, Japan).

The QuB program was used for 3D data mapping and experiment control and visualization, and for image acquisition with the camera (Navarro et al., 2015). 
ScanImage 3.8.1 (Vidrio Technologies, USA) was used for two-photon image acquisition (Pologruto et al., 2003). PatchMaster (HEKA, Germany) and QuB were used for VC and $\mathrm{CC}$ data acquisition, respectively. All computer work was done on a dual-processor workstation equipped with 8-core Opteron 4284 processors (AMD, USA) and an R9 280X Radeon video card (AMD, USA), running Windows 7 (Microsoft, USA).

\subsection{Results}

To investigate the role of intracellular $\left[\mathrm{Ca}^{2+}\right]$ on ventral raphé neuron activity in neonatal rats, we used whole-cell patch clamp to isolate and record voltage-gated sodium currents. The raphé neurons sit at the seam or midline of the in situ respiratory brainstem preparation (Fig. 4.2) and exhibit steady firing rates of 2-3 Hz, with broad action potentials (Fig. 4.2D). Raphé neurons are easy to identify and readily form gigaseals and are easy to voltage-clamp.

Voltage-gated $\mathrm{Na}^{+}$currents were isolated from other ionic currents by applying combinations of toxins within the intracellular solution and in the bath. The voltageclamp solution contains several $\mathrm{Kv}$ channel blockers: $\mathrm{Cs}^{+}$, TEA, and 4AP. These blockers, combined with $200 \mu \mathrm{M} \mathrm{CdCl}_{2}$ applied in the bath (Fig 4.3.A), resulted in good isolation of $\mathrm{Na}^{+}$currents. For further isolation of the Nav current, a saturating dose of TTX $(1 \mu \mathrm{M})$ was applied to the bath and protocols were repeated. Unlike VC solutions, CC solutions do not have any blockers, therefore the $\mathrm{Na}^{+}$current was isolated by TTX subtraction (Fig. 4.3B). TTX subtraction is highly dependent on being able to record stable currents prior and post TTX application. If the series resistance changes in time, 
the TTX subtraction will be subject to experimental artifacts by over- or under-estimating the total $\mathrm{Na}^{+}$current.

\subsubsection{Steady-state activation and inactivation}

To test whether the rhythm-generating transient $\mathrm{Na}^{+}$currents can be modulated by intracellular $\left[\mathrm{Ca}^{2+}\right]$, SS Activation and SS Inactivation properties were measured using the protocol shown in Fig. 4.4A. For each day of experiment, both solutions were prepared and pipettes were filled alternating solution each time to standardize acquisition. For each set of data, only the best most stable recordings were used. Examining the time course of activation, High $\left[\mathrm{Ca}^{2+}\right]$ neurons had slightly slower kinetics (Fig. 4.5B), compared to the Low $\left[\mathrm{Ca}^{2+}\right]$ solution, although when fitted with bi-exponential functions, the fast time constants corresponding to the initial phase of recovery do not differ (Fig. 4.5C; High $n=6$, low $n=6$ ). When fitting the decay of SS inactivation, High $\left[\mathrm{Ca}^{2+}\right]$ had slightly faster rates, but only by $<0.1 \mathrm{~ms}$ (Fig. 4.5D; Low $\mathrm{n}=9$, High $\mathrm{n}=9$ ), which potentially could be caused by series resistance compensation. The voltage dependence of activation was fit to a Boltzmann function multiplied by a line to account for the linear trend of the GV curve. The $\mathrm{V}_{\text {half }}$ or slope of SSAct did not vary significantly between Low $\left[\mathrm{Ca}^{2+}\right](-33 \pm 0.8 \mathrm{mV})$ and High $\left[\mathrm{Ca}^{2+}\right](-32 \pm 0.5 \mathrm{mV})$. Both data sets could be fit with the same $\mathrm{V}_{\text {half }}$ and slope parameters ( $\mathrm{p}>0.2$, F-test). However, the $\mathrm{V}_{\text {half }}$ of SSInact was more depolarized for Low $\left[\mathrm{Ca}^{2+}\right]$ solution $\mathrm{V}_{\text {half }}=-46.3 \pm 1.6 \mathrm{mV}(\mathrm{n}=9)$ compared to $\operatorname{High}\left[\mathrm{Ca}^{2+}\right], \mathrm{V}_{\text {half }}=-50.6 \pm 0.97 \mathrm{mV}(\mathrm{n}=9)$. There were no differences found in the peak current density or cell capacitance between the two data sets (Low, $n=28$; High, $n=21$ ), indicating that the two sample sets likely contain similarly sized neurons. 


\subsubsection{Recovery from inactivation}

The recovery from inactivation (RI) of $\mathrm{Na}^{+}$currents in ventral raphé neurons have a biexponential time course, indicating two potential mechanisms regulating the recovery (Milescu et al., 2010b). Only stable recordings where the amplitude of the first test pulse changed by $<12 \%$ from the first to the last sweep were included in the analysis. The recovery was tested at $-80 \mathrm{mV}$, where channel availability is $100 \%$, and at $-65 \mathrm{mV}$, where availability is incomplete, but the voltage is near the recorded resting potential of these neurons. I also attempted to test recovery at $-60 \mathrm{mV}$, but not all cells could be properly voltage clamped for the entire length of the protocol. For all RI traces, the data were first fit with a single exponential decay model. For each data set, the fits with a single exponential were poor and could not explain the slow time constant. The best biexponential fits to RI80 data had the following parameters, $\operatorname{Low}\left[\mathrm{Ca}^{2+}\right]: \tau_{\text {fast }}=6.28 \pm$ $0.39, \tau_{\text {slow }}=587.3 \pm 212.4 \mathrm{~ms}(\mathrm{n}=13) ; \mathrm{High}\left[\mathrm{Ca}^{2+}\right]: \tau_{\text {fast }}=6.11 \pm 0.4 \mathrm{~ms}$ and $\tau_{\text {slow }}=$ $379.3 \pm 139 \mathrm{~ms}(\mathrm{n}=17)$. The data sets could be fit equally well $\left(\mathrm{R}^{2}=0.93\right)$ with the same $\tau_{\text {slow }}=464 \pm 119 \mathrm{~ms}$, however when fit individually the $\operatorname{High}\left[\mathrm{Ca}^{2+}\right]$ had a faster $\tau_{\text {slow }}($ Fig. 4.6).

For RI-65, the recovery was not complete after 5 seconds, however both Low and High $\left[\mathrm{Ca}^{2+}\right]$, reached the same equilibrium value at the end of the protocol. Data sets that did not reach values greater than 0.8 were removed from the analysis. Representative VC traces are displayed in Fig. 4.6B. For Low $\left[\mathrm{Ca}^{2+}\right] \tau_{\text {fast }}=14.3 \pm 1.05 \mathrm{~ms}$ and $\tau_{\text {slow }}=556.9$ $\pm 254 \mathrm{~ms}(\mathrm{n}=17)$. For High $\left[\mathrm{Ca}^{2+}\right] \tau_{\text {fast }}=14.6 \pm 1.02 \mathrm{~ms}$ and $\tau_{\text {slow }}=537.3+/-221 \mathrm{~ms}(\mathrm{n}$ 
= 19). The overlap of the best fit values demonstrates that High intracellular $\left[\mathrm{Ca}^{2+}\right]$ does not modify recovery kinetics for either recovery voltage.

\subsubsection{Use-dependent inactivation}

As pacemaking neurons typically operate under repetitive firing, we tested the use dependence of inactivation, using fast $5 \mathrm{~ms}$ pulses from -80 to $-15 \mathrm{mV}$, repeated at different frequencies. To determine how many pulses are required to reach a steady state, we tested protocols with 100 pulses (Fig. 4.7). These data were collected under VC but with High $\left[\mathrm{Ca}^{2+}\right](\mathrm{n}=5)$ and Low $\left[\mathrm{Ca}^{2+}\right](\mathrm{n}=15) \mathrm{CC}$ solutions. The data could not be fit with a single exponential component, but required a bi-exponential. The general trend, as expected, is that, as the frequency of stimulation increases, the proportion of channels that become unavailable increases. Both sets of data could be fit with the same fast and slow time constants (Fig. 4.7). However, at $10 \mathrm{~Hz}$ and $20 \mathrm{~Hz}$, Low $\left[\mathrm{Ca}^{2+}\right]$ solutions had more channels become unavailable (more inactivation). The fast time constants of the data determined that the first component is complete within what would be the first 20 stimulus pulses.

To record properly isolated $\mathrm{Na}^{+}$currents, we designed $\mathrm{VC}$ protocols containing 20 pulses repeated at $2 \mathrm{~Hz}, 5 \mathrm{~Hz}$, and $12 \mathrm{~Hz}$, as these frequencies are within the normal firing range of raphé neurons. Similar to the RI protocol, we chose $-80 \mathrm{mV}$ and $-65 \mathrm{mV}$ as the interpulse voltages. With only 20 pulses, the decay in peak current can be fit with a single exponential and both Low and High solutions could be fit with the same time constant. With a holding voltages at $-65 \mathrm{mV}$, the time constants were estimated as follows: for 2 $\mathrm{Hz}, \tau=1.807 \pm 0.22 \mathrm{~s} ; 5 \mathrm{~Hz}, \tau=4.04 \pm 0.45 \mathrm{~s}$; and $12 \mathrm{~Hz}, \tau=8.82 \pm 0.93 \mathrm{~s}$. With 
holding voltages at $-80 \mathrm{mV}$, where there is little SS inactivation (SSInact in Fig. 4.5) the time constants were faster: for $2 \mathrm{~Hz}, \tau=1.21 \pm 0.57 \mathrm{~s} ; 5 \mathrm{~Hz}, \tau=2.33 \pm 0.32 \mathrm{~s}$; and $12 \mathrm{~Hz}$, $\tau=6.28 \pm 0.72 \mathrm{~s}$. As both sets of data could be fit to the same exponential and the only difference lies in the accumulation into inactivation, this behavior may be due simply to the $3 \mathrm{mV} \mathrm{V}_{\text {half }}$ shift of SS-inactivation between Low and High $\left[\mathrm{Ca}^{2+}\right]$.

\subsubsection{Recovery from use-dependent inactivation}

The use-dependence protocols demonstrated that the Low $\left[\mathrm{Ca}^{2+}\right]$ solutions accumulate more channels into a non-conducting inactivated state, which could be fit to a monoexponential process. As recovery from inactivation is a bi-exponential process, we designed voltage-clamp protocols that tested use-dependence and then examined the process of recovery from the use-dependence steady state. The protocols contained 20 pulses repeated at $5 \mathrm{~Hz}, 10 \mathrm{~Hz}$, and $20 \mathrm{~Hz}$, followed by a single test pulse applied after exponentially increasing time intervals. The initial recovery interval was $100 \mathrm{~ms}$, increasing exponentially for 10 sweeps, with the last interval 30 seconds. The intersweep interval had to be increased from $6 \mathrm{~s}$ to $30 \mathrm{~s}$ to allow time for full recovery, as having only a $6 \mathrm{~s}$ interval decreased the total available current dramatically with 20 pulses. These data were collected with High and Low $\left[\mathrm{Ca}^{2+}\right] \mathrm{CC}$ solutions, not with VC solutions. Fig. 4.9A shows an example protocol. Although sample sizes were low, the recovery after stimulus could be fit to a single exponential. In agreement with the use-dependence data, Low $\left[\mathrm{Ca}^{2+}\right]$ solutions induced greater accumulation into inactivation than the High $\left[\mathrm{Ca}^{2+}\right]$, allowing for faster recovery with the $\mathrm{High}\left[\mathrm{Ca}^{2+}\right]$. 


\subsection{Discussion}

As discussed in previous chapters, identifying the overall kinetics is ideally determined by fitting a model to the data. Initial modeling attempts were made for these voltageclamp experiments, but, as presented here, intracellular $\left[\mathrm{Ca}^{2+}\right]$ had minor effects on the kinetics of voltage-gated Nav channels in raphé neurons. Such slight effects on the kinetics makes it challenging to develop meaningful models that can be tested for comparison. If more differences in the kinetics had been found, creating a model that explains both data sets could lead to interesting dynamic clamp studies, which could potentially tease apart calcium dependence from activity dependence.

Our experimental observations about the recovery process could be explained by the slight rightward shift of the inactivation curve at $\mathrm{High}\left[\mathrm{Ca}^{2+}\right]$. In this case, at the same voltage near the top of the IV curve (e.g., at $-65 \mathrm{mV}$ ), High $\left[\mathrm{Ca}^{2+}\right]$ solutions would have a higher proportion of channels that are not inactivated compared to Low $\left[\mathrm{Ca}^{2+}\right]$ solutions. Therefore, it makes sense that the differences between the two data sets would be largest when the holding voltage was $<-80 \mathrm{mV}$. The effects that we saw here may not be due to any interaction with calcium, but rather to a simple shift in voltage dependence.

Prior to this study, I performed experiments with more extreme intracellular $\left[\mathrm{Ca}^{2+}\right]$ of nominally zero and $1 \mathrm{mM}$. Like many before me, I found it extremely challenging to patch in conditions with high $\left[\mathrm{Ca}^{2+}\right]$, and the zero $\mathrm{Ca}^{2+}$ had similar stability issues.

Therefore, I chose the two $\left[\mathrm{Ca}^{2+}\right]$ of $\sim 70 \mathrm{nM}$ and $\sim 7 \mu \mathrm{M}$, which represent more physiologically relevant extremes. Also, at around $6 \mu \mathrm{M}$, both calmodulin lobes should be fully bound to calcium, which should cause any effect - if there is one. Furthermore, 
EGTA is likely not the best buffer to use for establishing the Low $\left[\mathrm{Ca}^{2+}\right]$, as BAPTA can effectively buffer faster and at longer distances, whereas the spatial distribution of EGTA buffering may not reach the $\mathrm{Ca}^{2+}$ located in smaller processes (Pethig et al., 1989; Stern, 1992). Although my two $\mathrm{Ca}^{2+}$ solutions spanned two orders of magnitude, the difference may not have been enough to trigger any potential response. The ideal experiment would be to record voltage-clamp currents and then uncage calcium within the cell while recording the response to a use-dependence protocol. As our lab has a two-photon laser, I tried to uncage calcium using DM-nitrophen, a caged molecule with high two-photon cross absorption. A two-photon laser is useful to image deeply within a slice, but in many of my recordings it resulted in thermal gradients that would trigger uncontrolled action potentials. As the first laser pulse will uncage the highest dose of $\mathrm{Ca}^{2+}$, subsequent attempts to uncage after the uncontrolled action potentials failed to increase local $\left[\mathrm{Ca}^{2+}\right]$ sufficiently.

Another issue with this current study is the potential heterogeneity in the type of neurons that were tested, for which we did not have genetic markers available. Instead, I selected neurons based on their location and electrophysiological properties, but this reduced the sample size and made it more difficult to ascertain meaningful differences in the data. 


\subsection{Figures and Figure Legends}

\section{A}

DI

DII

DIII

DIV

$\beta$ subunit

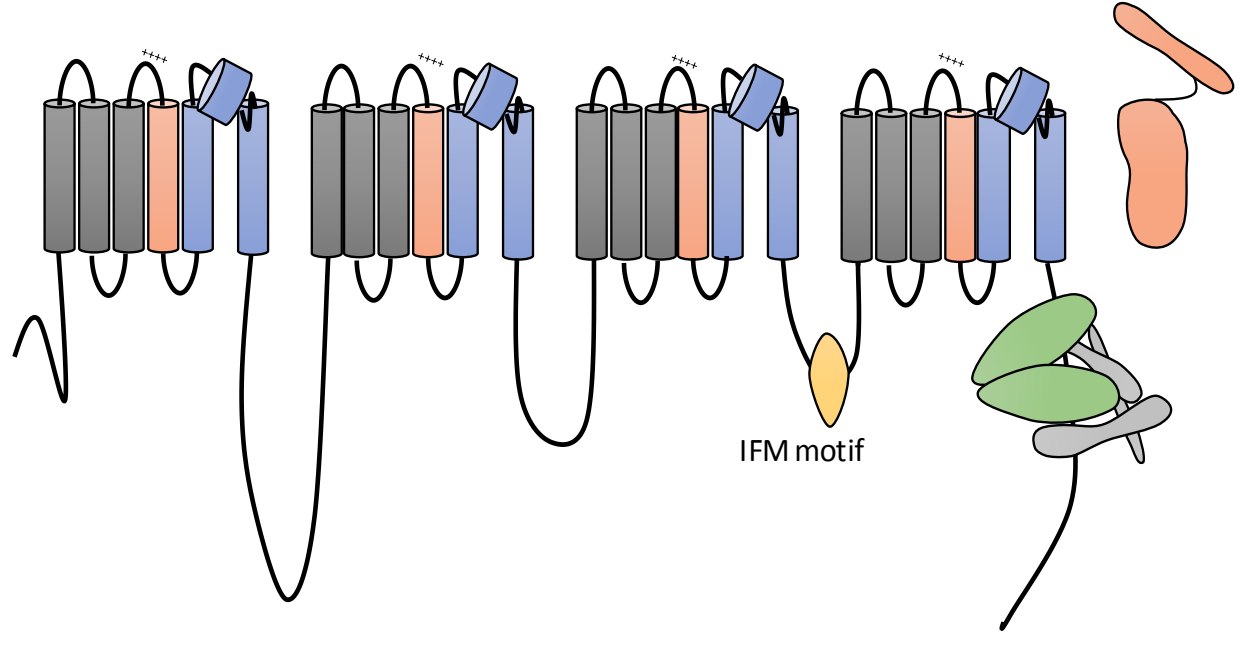

Figure 4.1. Schematic of Nav channel secondary structure. The Nav $\alpha$-subunit has four domains interconnected by intracellular linkers. Each domain has six transmembrane segments (S1 through S6). The S4 segment (red) has positively charged arginines that are translocated within the membrane as voltage is depolarized. S5-S6 from each domain (blue) form the ion conducting pore. The DIII - DIV linker contains the IFM motif (yellow), which is the inactivation "particle" responsible for fast inactivation. The C-terminus contains binding sites for regulatory factors, including calmodulin (green) and fibroblast growth factor homologous factors (grey). Nav $\alpha$-subunits are typically expressed with at least one $\beta$ subunit (orange), which are single transmembrane subunits with an extracellular immunoglobulin motif. 

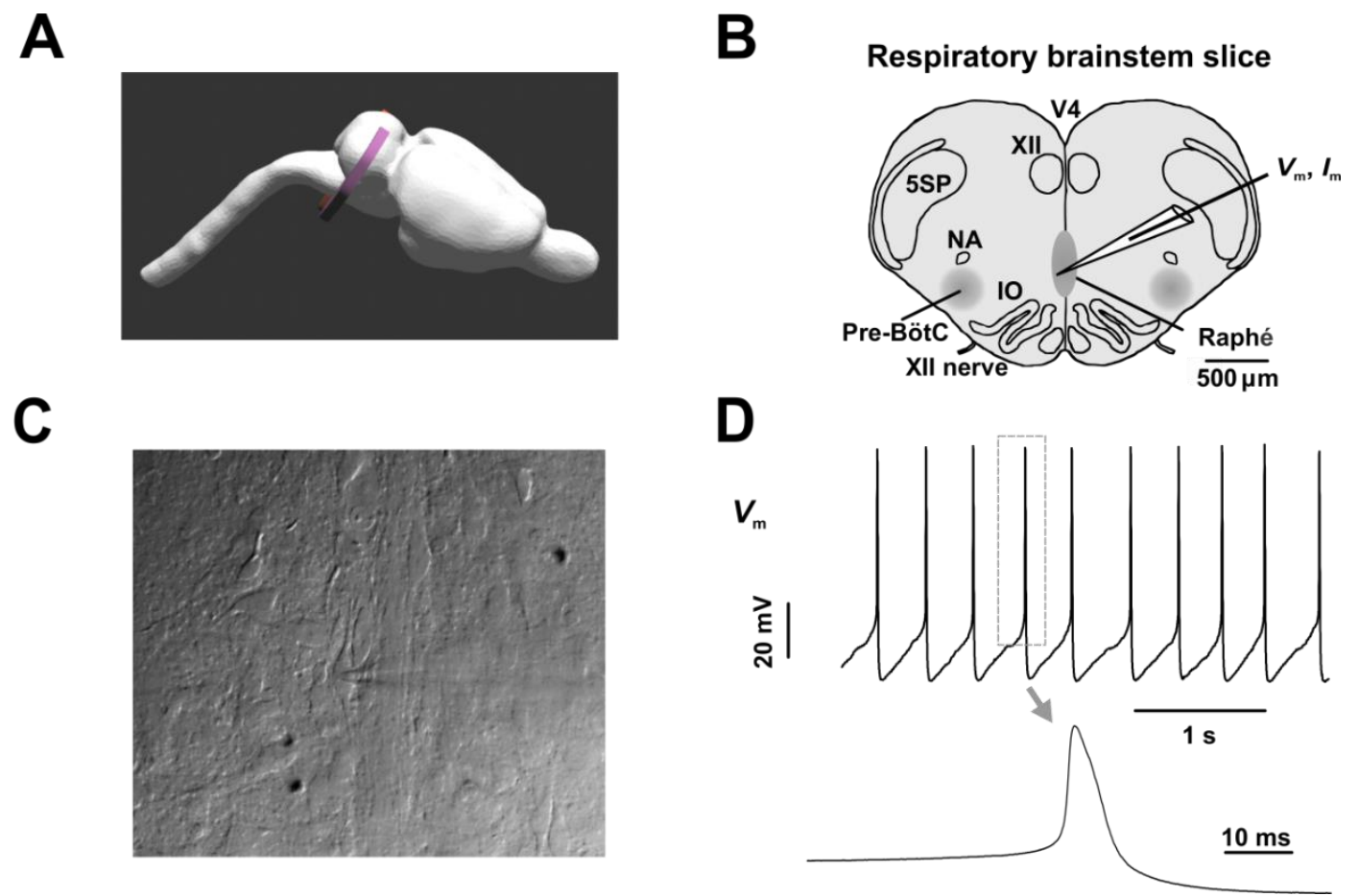

Figure 4.2. The respiratory brainstem preparation. A) 3D model of the rat brain loaded in QuB's World3D viewer viewer. The purple marker indicates the location of the slice used in our experiments. B) Schematic representation of the respiratory brainstem slice (350-400 $\mu \mathrm{m}$ thick) of the neonatal rat, containing the inspiratory rhythm-generating circuits in the Pre-Bötzinger complex (Pre-BotC), with output to the hypoglossal motor nucleus (XII). Other important structural features include the inferior olivary nucleus (IO), nucleus ambiguus (NA), spinal trigeminal nucleus (5SP), and the wall of the fourth ventricle (V4). C) IR Dodt-contrast image of a patched raphé neuron located along the midline. D) Typical steady firing rate $(2-3 \mathrm{~Hz})$ of raphé neurons, with characteristic broad calcium shoulder after the peak of the action potential. 
A

$200 \mu \mathrm{M} \mathrm{CdCl}$
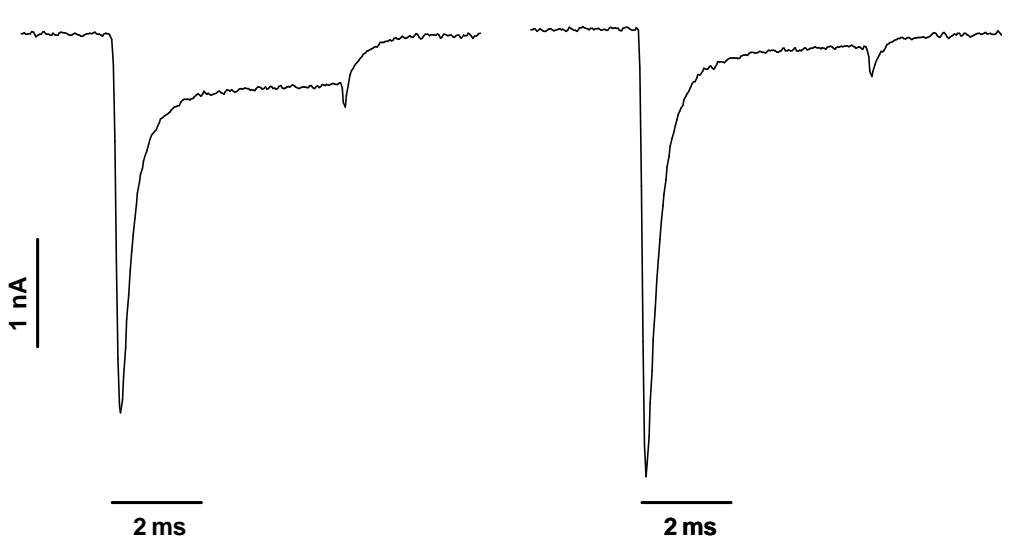

B

$1 \mu \mathrm{M} T \mathrm{TX}$

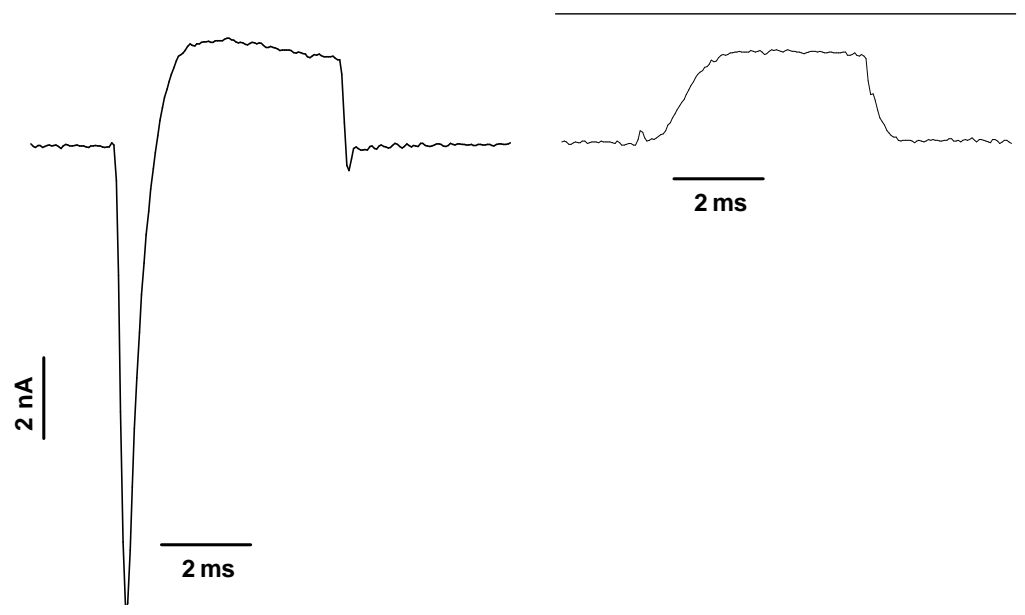

Figure 4.3. Isolation of $\mathrm{Na}^{+}$currents. A) When using VC solutions in VC experiments, voltage pulses from -80 to $-15 \mathrm{mV}$ elicit inward $\mathrm{Na}^{+}$and $\mathrm{Ca}^{2+}$ currents. Under bath-applied $200 \mu \mathrm{M}$ $\mathrm{CdCl}_{2}$, the inward $\mathrm{Ca}^{2+}$ currents are eliminated. B) When using CC solutions in VC experiments, pulses from -80 to $-15 \mathrm{mV}$ elicit both a $\mathrm{Na}^{+}$-dominated inward current and a $\mathrm{K}^{+}$-outward current $\left(\mathrm{K}^{+}\right)$. After TTX application, the inward current is mostly removed, leaving only small $\mathrm{Ca}^{2+}$ current masked by the large $\mathrm{K}^{+}$current. 
A
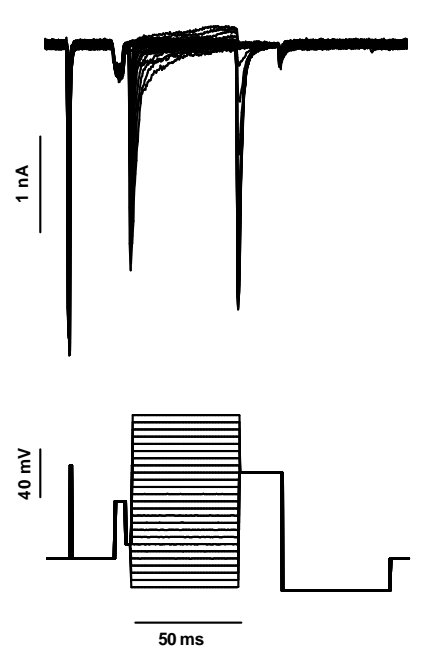

B
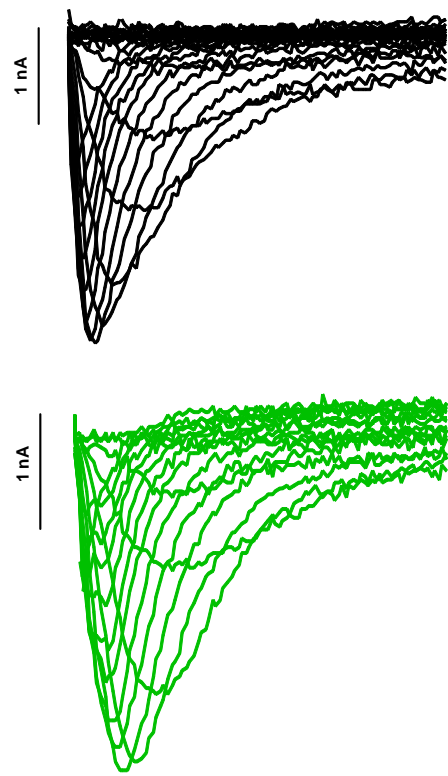

sme
C
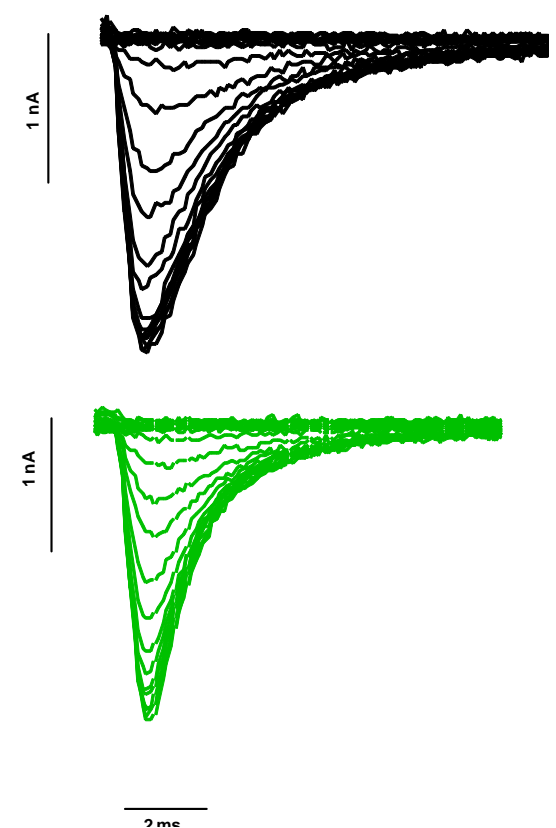

Figure 4.4. Testing activation and inactivation properties. A) Full voltage clamp protocol used to test the SS-Act and SS-InAct. B) Representative traces of the time course of Activation, topblack - Low $\left[\mathrm{Ca}^{2+}\right]$ solution, bottom - green High $\left[\mathrm{Ca}^{2+}\right]$ solution. C) Representative traces of the time course of inactivation, top-black - EGTA low calcium solution, bottom - green HEDTA high $\mathrm{Ca}^{2+}$ solution. The bottom scale bar represents $2 \mathrm{~ms}$. 
A

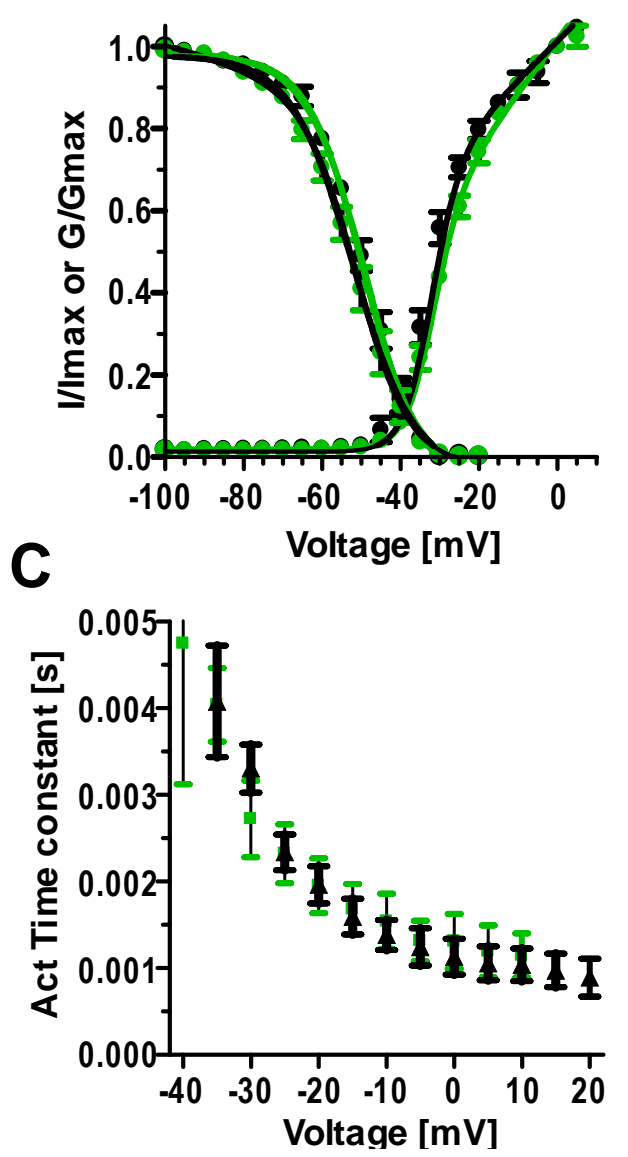

B

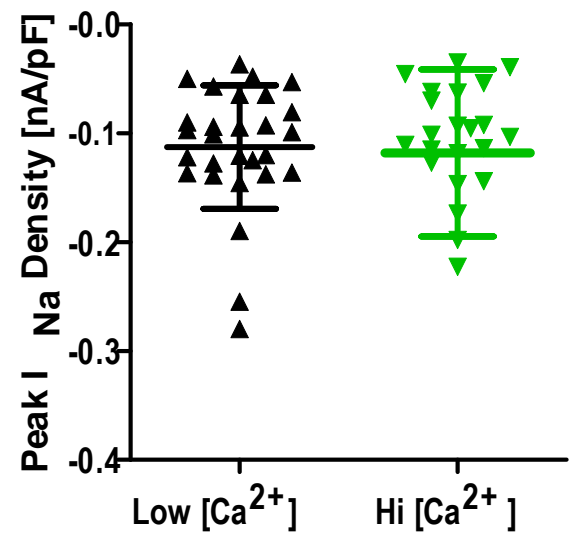

D

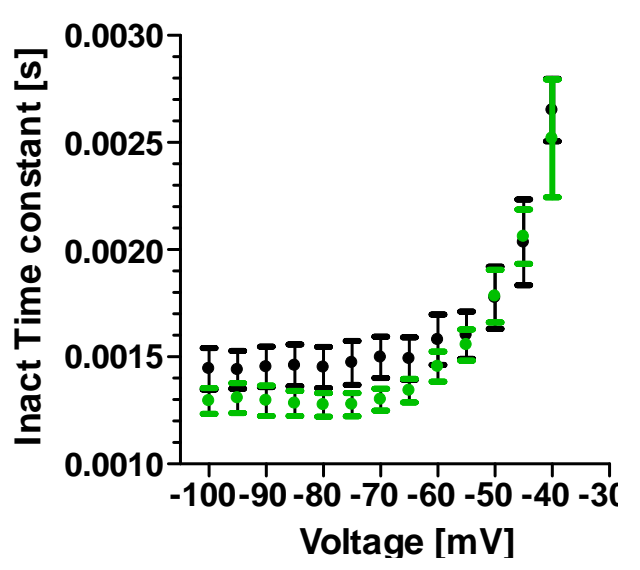

Figure 4.5. Steady-state activation and inactivation kinetics. A) The SS-Act G/V curves were fit with Boltzmann equations multiplied by a line. The $\mathrm{V}_{\text {half }}$ and slope of SSAct did not differ significantly ( $\mathrm{p}$ value $>0.2$ ) between the two data sets, Low $\left[\mathrm{Ca}^{2+}\right]=-33 \pm 0.8 \mathrm{mV}, 3.73 \pm 0.35, \mathrm{n}$ $=6$; High $\left[\mathrm{Ca}^{2+}\right]=-32 \pm 0.5 \mathrm{mV}, 3.73 \pm 0.35, \mathrm{n}=6$. The SS-Inact $\mathrm{I} / \mathrm{V}$ curves were fit with Boltzmann equations, showing that the $\mathrm{V}_{\text {half }}$ for Low $\left[\mathrm{Ca}^{2+}\right]$ solutions was shifted in the depolarizing direction: $V_{\text {half }}$, slope $=-46.3 \pm 1.6 \mathrm{mV},-7.74 \pm 0.51$ compared to $V_{\text {half }}=-50.6 \pm 0.97$ $\mathrm{mV},-6.811 \pm 0.72$. B) The peak $\mathrm{Na}^{+}$current density was calculated from the maximum current elicited by a voltage pulse from -80 to $-15 \mathrm{mV}$, divided by the estimated $\mathrm{C}_{\text {slow }}$. There were no differences between the two data sets (Low, $n=28$; High, $n=21$ ). C) The plot of the time constants of SSAct decay vs. voltage (Low, $n=6$; High $n=6$ ). D) The plot of SSInact decay time constants vs. voltage demonstrates that inactivation kinetics are slightly faster with High $\left[\mathrm{Ca}^{2+}\right]$ solutions (Low, $n=9$; High, $n=9$ ). 
A
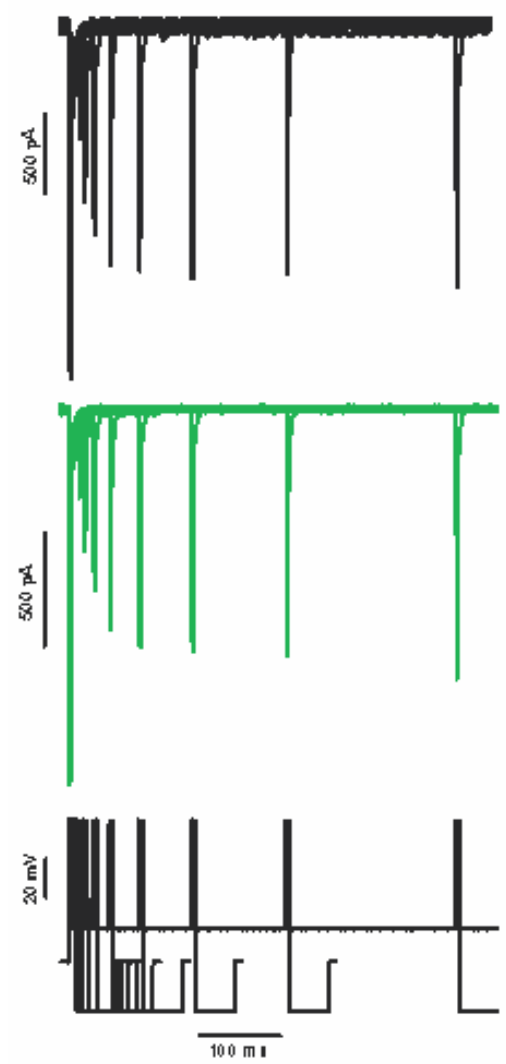

B
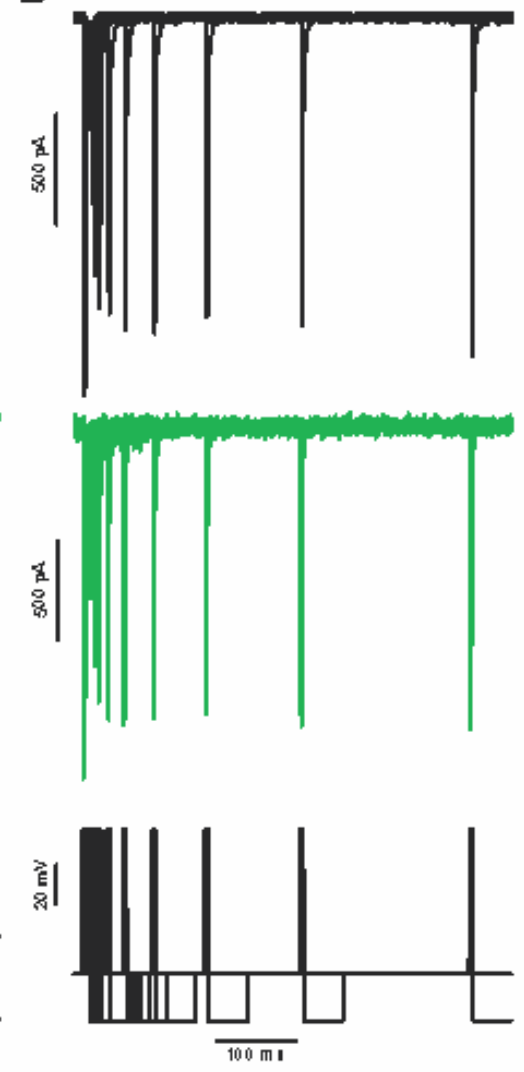

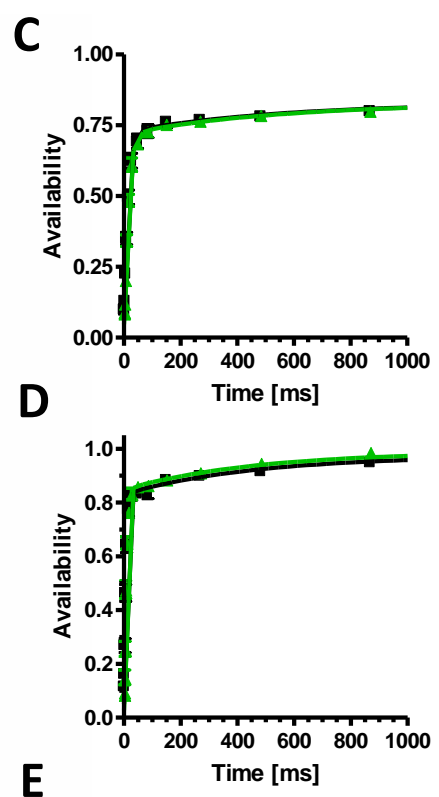

E

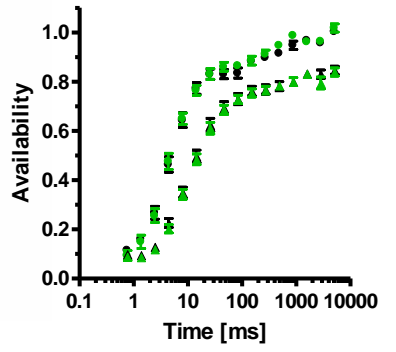

Figure 4.6. Recovery from inactivation of $\mathbf{N a}^{+}$currents. A) Representative traces of raw recovery protocol at $-65 \mathrm{mV}$. The current does not fully recover for the length of the protocol. Top, black trace - Low $\left[\mathrm{Ca}^{2+}\right]$. Bottom, green trace $-\mathrm{High}\left[\mathrm{Ca}^{2+}\right]$. B) Representative traces of the raw recovery protocol at $-80 \mathrm{mV}$. Top, black trace - Low $\left[\mathrm{Ca}^{2+}\right]$. Bottom, green trace - High $\left[\mathrm{Ca}^{2+}\right]$. C) The recovery property curve for $-65 \mathrm{mV}$ holding voltage, with the corresponding fits. Only the first second of data is shown, to showcase the bi-exponential time course. D) The recovery property curve for $-80 \mathrm{mV}$ with corresponding fits. E) Same data as in C and D, except plotted on an exponential time course 

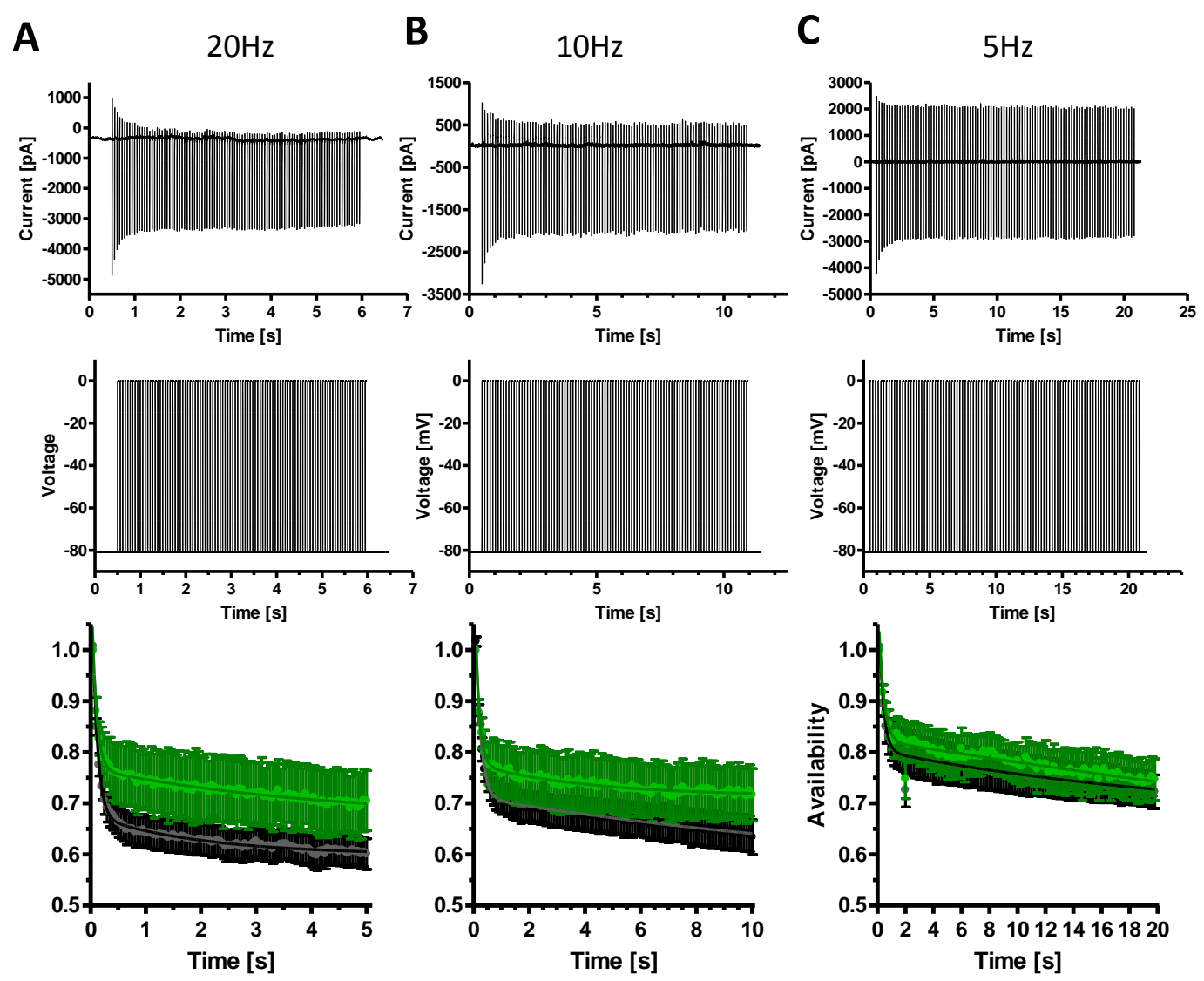

Figure 4.7. Use-dependent inactivation tested with 100 pulses. These data were collected in VC, but with CC solutions without blocking $\mathrm{Ca}^{2+}$ currents. A) 100 pulses at $20 \mathrm{~Hz}$. B) 100 pulses at $10 \mathrm{~Hz}$. C) 100 pulses at $5 \mathrm{~Hz}$. The top trace in each graph is the elicited current, below is the voltage, and the bottom is the analyzed data, where green represents $\mathrm{High}\left[\mathrm{Ca}^{2+}\right](\mathrm{n}=5)$ and black represents Low $\left[\mathrm{Ca}^{2+}\right](\mathrm{n}=14)$. At higher pulse repetition rates, the entry into inactivation is enhanced, as indicated by the change in overall steady state. The data were fit to bi-exponential functions. The estimated time constants were statistically compared between data sets and no significant differences were found: for $5 \mathrm{~Hz}, \tau_{\text {fast }}=0.029 \pm 0.056 \mathrm{~s}, \tau_{\text {slow }}=3.16 \pm 0.93 \mathrm{~s}$; for $10 \mathrm{~Hz}$, $\tau_{\text {fast }}=0.082 \pm 0.13 \mathrm{~s}, \tau_{\text {slow }}=5.62 \pm 1.08 \mathrm{~s}$; for $20 \mathrm{~Hz}, \tau_{\text {fast }}=0.456 \pm 0.31 \mathrm{~s}, \tau_{\text {slow }}=11.1 \pm 2.03 \mathrm{~s}$. However, with repetition frequencies above $10 \mathrm{~Hz}$, the Low $\left[\mathrm{Ca}^{2+}\right]$ solution gives a larger fraction of inactivated channels. The first exponential component reaches a steady state level within the first 20 pulses, followed by a steady decay. 

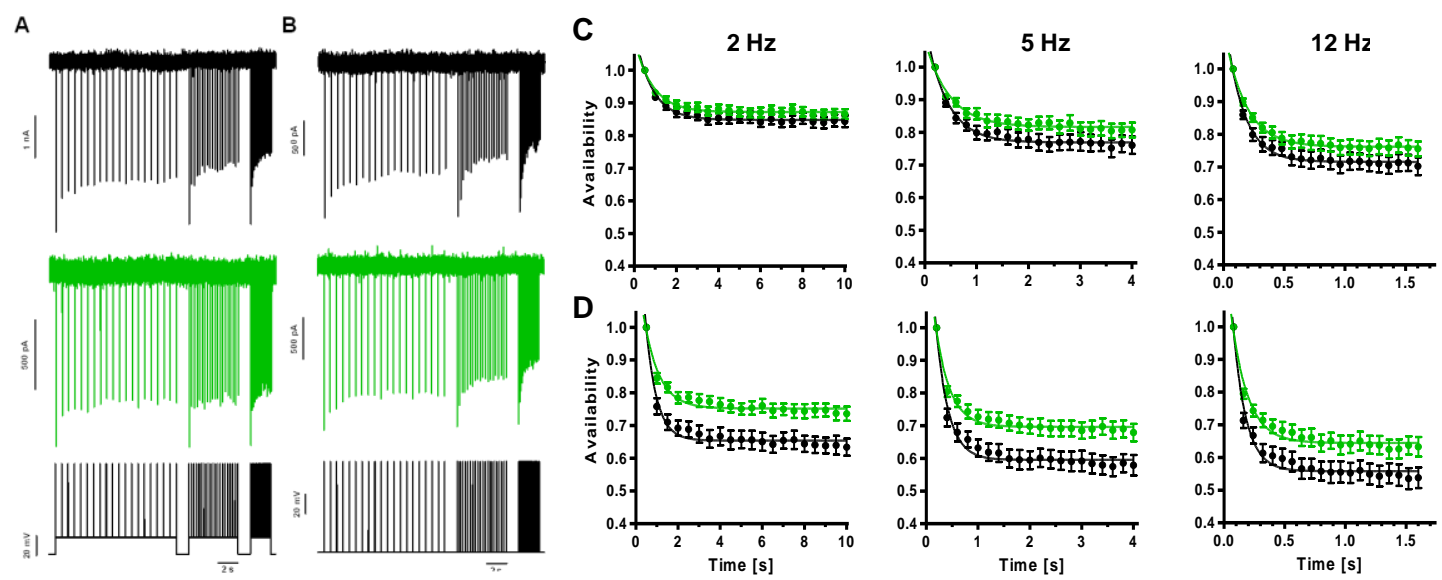

Figure 4.8. Use-dependent inactivation with voltage-clamp solutions. A) Representative traces for frequency stimulation at $-65 \mathrm{mV}$, for both Low $\left[\mathrm{Ca}^{2+}\right]$ (black) $(\mathrm{n}=17)$ and High $\left[\mathrm{Ca}^{2+}\right]$ (green) ( $\left.\mathrm{n}=10\right)$ solutions. B) The same as in A, but at $-80 \mathrm{mV}(\mathrm{n}=19$ for both High and Low ). C) Time course of use-dependent inactivation at $-80 \mathrm{mV}$. The current evoked by a given pulse was normalized to the current evoked by the first pulse. D) Same as in C, but at $-65 \mathrm{mV}$. Pulse trains with only 20 pulses could be fit to a single-exponential function. No statistical difference was detected between -80 and $-65 \mathrm{mV}$, but the plateau was significantly different, with Low $\left[\mathrm{Ca}^{2+}\right]$ solutions having a higher fraction of inactivated channels. These data are in agreement with the data recorded with CC solutions. 
A

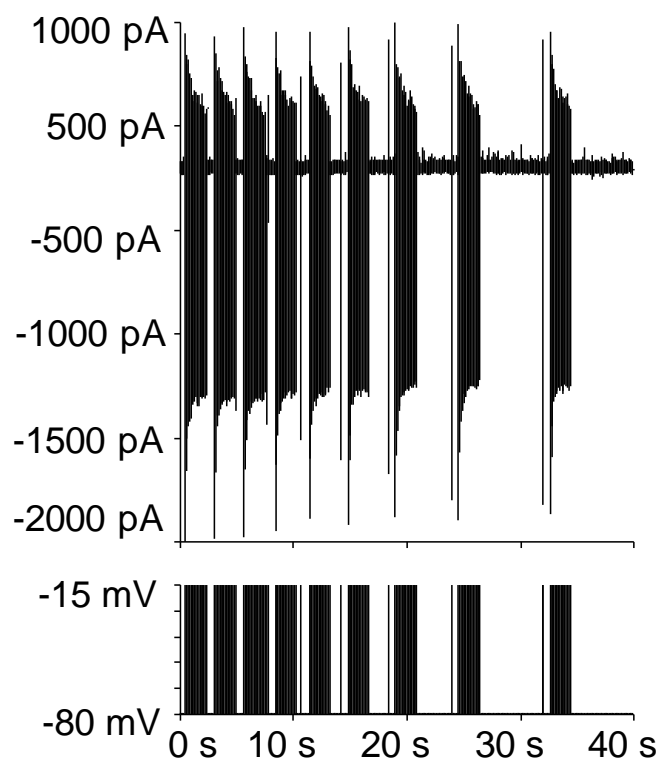

B
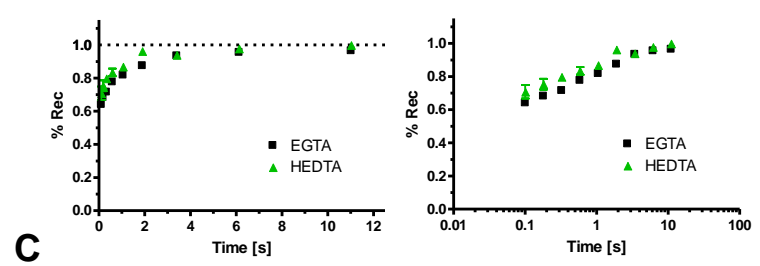

C
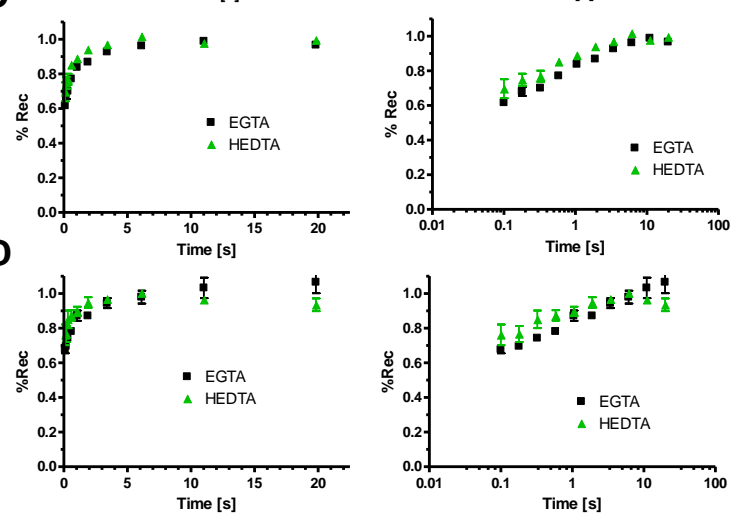

Figure 4.9. Recovery after use-dependent inactivation. A) Representative trace of a protocol with 20 pulses repeated at either $5 \mathrm{~Hz}, 10 \mathrm{~Hz}$, or $20 \mathrm{~Hz}$, followed by a single test pulse with exponentially increasing interval. If the intersweep interval was less than 30 seconds, the 20 pulse trains did not reach the same steady state but continued to inactivate. B) Analyzed recovery for 20Hz: Left, linear time scale; Right, exponential time scale. Low $\left[\mathrm{Ca}^{2+}\right]$ or EGTA $(n=6)$, High $\left[\mathrm{Ca}^{2+}\right](\mathrm{n}=4)$. C) Same as in B for $10 \mathrm{~Hz}$. Low $\left[\mathrm{Ca}^{2+}\right](\mathrm{n}=2)$, High $\left[\mathrm{Ca}^{2+}\right](\mathrm{n}=3)$. D) Same as in B for $5 \mathrm{~Hz}$. Low $\left[\mathrm{Ca}^{2+}\right](\mathrm{n}=4) \mathrm{High}\left[\mathrm{Ca}^{2+}\right](\mathrm{n}=2)$. The sample sizes for this data set are low as many samples had to be discarded due to the 20 pulses reaching a similar steady state. 


\title{
Chapter 5
}

\section{Sodium channels implement a molecular leaky integrator that detects action potentials and regulates neuronal firing}

This chapter represents unpublished work.

\begin{abstract}
Voltage-gated sodium channels play a critical role in cellular excitability, amplifying a small membrane depolarization into a full blown action potential. Interactions with auxiliary subunits and factors modify the intrinsic kinetic mechanism to result in new molecular and cellular functionality. Here, we show that sodium channels can implement a molecular leaky integrator, where the input signal is the membrane potential and the output signal is the occupancy of a long-term inactivated state. Through this mechanism, sodium channels effectively measure the frequency of action potentials and convert it into $\mathrm{Na}^{+}$current availability. In turn, the $\mathrm{Na}^{+}$current can control firing frequency in a negative feedback loop. We present these ideas in the context of serotonergic raphé neurons, which fire spontaneously at low frequency and provide critical neuromodulation to many autonomous and cognitive brain functions.
\end{abstract}

Author contributions: M.A.N performed experiments, analyzed the data and wrote and edited the manuscript. 


\subsection{Introduction}

Computation in the brain begins at the molecular level, with proteins such as ion channels and receptors that can change their structural and functional state in response to changes in the environment. These molecular building blocks capable of processing information have been adapted by nature into progressively more complex computational structures: ion channels and receptors were incorporated into synapses and neurons, neurons were interconnected into networks and circuits, and circuits were made into a brain capable of abstract thinking. Not surprisingly, computation in the engineering world followed the same trend: transistors were combined into integrated circuits, circuits were assembled into microprocessors, and microprocessors were developed into computers.

At the molecular level, voltage-gated sodium (Nav) channels have long been credited with a critical role in cellular excitability: amplifying a small membrane depolarization, such as created by a tiny postsynaptic excitatory current, into a full blown action potential (Hodgkin and Huxley, 1952). Computationally, Nav channels can be regarded as the equivalent of a transistor (Sigworth, 2003), a nonlinear electric circuit element. To generate action potentials of specific shape and firing patterns, spontaneously or in response to synaptic input, a neuron expresses a complement of Nav and other types of ion channels (Bean, 2007) and positions them strategically at subcellular locations (Kole and Stuart, 2012). Although we generally understand how ion channels contribute to the mechanics of action potential generation and propagation, the molecular and cellular landscapes are complex and remain incompletely charted. At the most basic level, we do 
not fully understand how ion channels function as molecular computational machines and how they interact with each other and with other factors to regulate cellular activity.

Here, we examine a new computational function of Nav channels that emerges from a process of long-term inactivation (LTI), which can be caused by interaction with fibroblast growth factor-homologous factors (FHFs), a relatively recently discovered group of auxiliary factors, reviewed by Goldfarb (Goldfarb, 2012). We are particularly interested in this functionality in the context of pacemaker serotonergic raphé neurons (Jacobs and Azmitia, 1992), which provide critical neuromodulation to many brain areas involved in autonomous (ventral raphé) and cognitive (dorsal raphé) functions. In a previous study (Milescu et al., 2010b), we examined the Nav channels in ventral raphé neurons and proposed a kinetic model that explains not only their intrinsic kinetic properties but also their characteristic process of long-term inactivation. Here, we investigate the computational aspects of this mechanism, using a combination of electrophysiology experiments and mathematical analysis.

\subsection{Materials and methods}

All animal procedures were approved by the Animal Care and Use Committees of the University of Missouri and SUNY Downstate Medical Center.

\section{$\underline{\text { 5.2.1 Brainstem slices }}$}

In vitro medullary slices containing ventral raphé neurons were obtained from neonatal (postnatal days 1 - 4) Sprague Dawley male and female rats, as described (Koshiya and Smith, 1999). Briefly, animals were anaesthetized with isoflurane and the brainstem was 
swiftly removed in artificial cerebral spinal fluid (aCSF) containing the following (in $\mathrm{mM}): 124 \mathrm{NaCl}, 25 \mathrm{NaHCO}_{3}, 3 \mathrm{KCl}, 1.5 \mathrm{CaCl}_{2}, 1 \mathrm{MgSO}_{4}, 0.5 \mathrm{NaH}_{2} \mathrm{PO}_{4}$, and $30 \mathrm{D}-$ glucose, equilibrated with $95 \% \mathrm{O}_{2}$ and $5 \% \mathrm{CO}_{2}(\mathrm{pH} 7.4 \pm 0.05$ at room temperature). Transverse slices (300-400 $\mu \mathrm{m}$ thick) containing nucleus raphé obscurus, the preBötzinger Complex, and hypoglossal (XII) nerve rootlets were cut on a Campden Instruments 7000 vibratome (Campden Instruments, England) and transferred to the recording chamber and superfused with $\mathrm{aCSF}$ at room temperature, at a rate of $\approx 5$ $\mathrm{ml} / \mathrm{min}$. Raphé neurons were generally identified based on their location in the slice, adjacent to the midline. Whenever possible, the neurons were further selected based on their spiking pattern: regular and slow pacemaking $(1-3 \mathrm{~Hz})$ present in cell-attached mode, and broad action potentials (3 - $6 \mathrm{~ms}$ ) with prominent calcium shoulder (Ptak et al., 2009). Whole-cell patch-clamp was done under IR-Dodt contrast imaging, using a Hamamatsu Flash 4.0 camera (Hamamatsu Photonics, Japan) controlled by the QuB software (Navarro et al., 2015).

\subsubsection{Acutely dissociated neurons}

Dorsal raphé neurons were acutely dissociated from adult male Sprague-Dawley rats (200-250 g), as described (Penington et al., 1991). Briefly, animals were anaesthetized with isoflurane and then decapitated with a small animal guillotine. A small volume of gray matter was cut from immediately below the cerebral aqueduct containing the dorsal raphé nuclei, chopped into pieces, and bathed for two hours at room temperature, in a PIPES buffer solution containing $0.2 \mathrm{mg} / \mathrm{mL}$ trypsin (Sigma Type XI) under pure oxygen. The tissue was then triturated in Dulbecco's modified Eagles's medium to free 
individual neurons. Small droplets containing suspended neurons were placed in the recording chamber and cells were allowed to settle and adhere to the bottom of the chamber. An extracellular recording solution containing (in $\mathrm{mM}$ ): $120 \mathrm{NaCl}, 10 \mathrm{TEACl}$, 20 HEPES, 30 sucrose, $3 \mathrm{KCl}, 1.5 \mathrm{CaCl}, 1 \mathrm{MgCl}_{2}(\mathrm{pH} 7.4 \pm 0.05$ with $\mathrm{CsOH}$ at room temperature) was continuously perfused at a rate of $\approx 2 \mathrm{ml} / \mathrm{min}$. Neurons with truncated dendrites and soma $\geq 20 \mu \mathrm{m}$ were selected for whole-cell patch-clamp.

\section{$\underline{\text { 5.2.3 Solutions }}$}

For voltage clamp (VC) in brain slices, pipettes were filled with a solution containing (in mM): 70 Cs-gluconate, 30 Na-gluconate, 10 tetraethylammonium-Cl (TEA-Cl), 5 4aminopyridine (4-AP), 10 EGTA, $1 \mathrm{CaCl}_{2}$, 10 HEPES, 4 Mg-ATP, $0.3 \mathrm{Na}_{3}$-GTP, $10 \mathrm{Na}_{2}$ phosphocreatine, $\mathrm{pH} 7.4$ with $\mathrm{CsOH}(285 \pm 5 \mathrm{mOsm} / \mathrm{L})$. For $\mathrm{VC}$ in dissociated neurons, the pipette solution contained (in $\mathrm{mM}$ ): $90 \mathrm{Cs}$-gluconate, $30 \mathrm{NaCl}, 10 \mathrm{TEA}-\mathrm{Cl}, 5$ 4-AP, 20 HEPES, 10 EGTA, $1 \mathrm{CaCl}_{2}$, 4 Mg-ATP, $0.3 \mathrm{Na}_{3}$-GTP, $10 \mathrm{Na}_{2}$-phosphocreatine, $\mathrm{pH}$ 7.4 with $\mathrm{CsOH} . \mathrm{Cs}^{+}, \mathrm{TEA}^{+}$, and 4-AP minimized $\mathrm{K}^{+}$currents, whereas the elevated $\mathrm{Na}^{+}$ concentration decreased the size of $\mathrm{Na}^{+}$currents and reduced VC artifacts. For current clamp (CC) and dynamic clamp (DC) in brain slices, pipettes were filled with a solution containing (in mM): $125 \mathrm{~K}$-gluconate, $4 \mathrm{NaCl}, 10 \mathrm{EGTA}, 1 \mathrm{CaCl}_{2}, 10 \mathrm{HEPES}, 4 \mathrm{Mg}$ ATP, $0.3 \mathrm{Na}_{3}$-GTP, $4 \mathrm{Na}_{2}$-phosphocreatine, pH 7.4 adjusted with $\mathrm{KOH}(285 \pm 5$ $\mathrm{mOsm} / \mathrm{L})$. For $\mathrm{VC}$ in brain slices, $\mathrm{CdCl}_{2}(200 \mu \mathrm{M})$ and 7-nitro-2,3-dioxo-1,4dihydroquinoxaline-6-carbonitrile (CNQX; $20 \mu \mathrm{M})$ were added to the superfusing aCSF to block $\mathrm{Ca}^{2+}$ currents and inhibit synaptic transmission. $\mathrm{CdCl}_{2}(200 \mu \mathrm{M})$ was also used for $\mathrm{VC}$ in dissociated neurons, while CNQX $(20 \mu \mathrm{M})$ was used for CC and DC 
experiments in brain slices. To block $\mathrm{Na}^{+}$currents in brain slices, tetrodotoxin (TTX, 1 $\mu \mathrm{M})$ was added to the superfusing aCSF. All reagents were purchased from MilliporeSigma (St. Louis, MO, USA), with the exception of Cs-gluconate from Hello Bio Inc (Princeton, NJ, USA) and TTX from Alomone Labs (Jerusalem, Israel).

\subsubsection{Electrophysiology}

For brain slices, pipettes (5 - $7 \mathrm{M} \Omega$ ) were pulled from borosilicate glass. For dissociated neurons, pipettes (2 - $3 \mathrm{M} \Omega$ ) were pulled from soda-lime glass. All pipettes were coated with Sylgard to reduce capacitive transients. Pipette capacitance was compensated $100 \%$ in $\mathrm{VC}$ and $\approx 75 \%$ in $\mathrm{CC}$ and DC. For DC and offline analysis, membrane capacitance $\left(\mathrm{C}_{\mathrm{m}}\right)$ was approximated as the value used for compensation in $\mathrm{VC}$ and was typically 20 $\mathrm{pF}$. Series resistance $\left(\mathrm{R}_{\mathrm{s}}\right)$ was typically $9-15 \mathrm{M} \Omega$. Cells with $\mathrm{Rs} \approx 15 \mathrm{M} \Omega$ or with evidence of poor space-clamp were discarded. In VC experiments, Rs was compensated $80 \%$ and the compensation was readjusted before running a protocol. In CC and DC experiments, Rs was compensated $100 \%$ and periodically readjusted. Measured liquid junction potentials of $\approx 10 \mathrm{mV}$ for the $\mathrm{K}^{+}$-based and $\approx 8 \mathrm{mV}$ for the $\mathrm{Cs}^{+}$-based solutions were corrected online. For neurons in the slice preparation, whole-cell recordings were obtained with an EPC-10 patch-clamp amplifier (HEKA Electronik, Germany), controlled by Patchmaster 2x90.2 software (HEKA Elektronik, Germany). For VC, the recorded currents were low-pass filtered at $40 \mathrm{kHz}$ and digitally sampled at $100 \mathrm{kHz}$. For $\mathrm{CC}$ and $\mathrm{DC}$, the membrane voltage signal was digitally sampled at $50 \mathrm{kHz}$ (openbandwidth). For acutely dissociated neurons, an Axopatch 200B patch-clamp amplifier (Molecular Devices, USA) was used, controlled by pClamp 10.3 software (Molecular 
Devices, USA). In this case, the recorded currents were low-pass filtered at $10 \mathrm{kHz}$ and digitally sampled at $50 \mathrm{kHz}$.

\section{$\underline{\text { 5.2.5 Voltage clamp experiments }}$}

Voltage clamp protocols (Fig. 5.2) were constructed and applied with the Patchmaster program (brainstem slices) or with pClamp 10.3 (acutely dissociated neurons). The intersweep interval was $6 \mathrm{~s}$ at $-80 \mathrm{mV}$, necessary for complete recovery from inactivation of $\mathrm{Na}^{+}$currents. Recordings with evidence of $\mathrm{Na}^{+}$current instability were discarded. Leak currents were subtracted using the $\mathrm{P} / \mathrm{n}$ procedure. For $\mathrm{VC}$ recordings in brain slices, the TTX-sensitive $\mathrm{Na}^{+}$current was isolated via TTX subtraction.

\section{$\underline{\text { 5.2.6 Nav kinetic model }}$}

For dynamic clamp experiments and computer simulations, we used the Nav model shown in Fig. 5.3A, using a previously published set of rate constant parameters (Model II in Supplemental Table 1 from (Milescu et al., 2010b). Each rate constant has an Eyring expression defined as $k=k^{0} \times e^{k^{1} \times V}$, where $k^{0}$ and $k^{1}$ are pre-exponential and exponential factors, respectively, and $V$ is membrane potential.

\subsubsection{Dynamic-clamp experiments}

To inject Nav conductance in live neurons, under bath-applied TTX, we used the dynamic clamp functionality in the QuB software (Milescu et al., 2008), following previously described procedures (Milescu et al., 2010b). The real-time computational loop was run at $50 \mathrm{kHz}$ and the Nav model was solved using the matrix method. The 
software was run on a dual-processor workstation with Xeon E5-2667 v2 8-core CPUs, running Windows 7, interfaced with the patch-clamp amplifier via a National Instruments data acquisition board PCIe-6361 and BNC-2120 connector block.

\section{$\underline{\text { 5.2.7 Computer simulations }}$}

To simulate the response of the model to voltage clamp protocols, we used the MLab edition of the QuB program (http://milesculabs.biology.missouri.edu/QuB), as previously described (Milescu et al., 2008; Milescu et al., 2010b). All data were analyzed with Prism 4.1 (GraphPad, CA, USA).

\subsection{Results}

\section{$\underline{\text { 5.3.1 Action potentials in serotonergic raphé neurons and the contribution of }}$}

\section{$\underline{\text { Nav channels }}$}

Action potentials vary in their properties in different neuronal types but they generally last from hundreds of microseconds to several milliseconds, rapidly swinging the membrane between hyperpolarized and depolarized states. Serotonergic neurons in either the ventral or dorsal raphé nuclei have a particular electrophysiological profile characterized by regular and spontaneous spiking at low frequency $(1-5 \mathrm{~Hz})$, a steady depolarization in the interspike interval, and broad action potentials ( 3 - $6 \mathrm{~ms}$ ), as illustrated in Fig. 5.1A and B. These characteristics are partially shared with other monoaminergic neurons (Grace and Bunney, 1983; Vandermaelen and Aghajanian, 1983; Li and Bayliss, 1998; de Oliveira et al., 2010; Tuckwell and Penington, 2014). 
As in most excitable cells, Nav channels play a central role in serotonergic neurons, releasing the large depolarizing $\mathrm{Na}^{+}$current $\left(\mathrm{I}_{\mathrm{Na}}\right)$ that generates the action potential (Milescu et al., 2010b). To perform their duty, Nav channels must cycle through a sequence of functional states, as summarized in Fig. 5.1C: they are (virtually) closed (C) in the interspike interval, activate and abruptly open $(\mathrm{O})$ and then quickly inactivate (I) during the action potential, and then recover from inactivation in the interspike interval. Interestingly, as it recovers from inactivation, the channel bypasses the open state, instead following the transition pathway indicated by the blue arrow in the figure. The resulting hysteresis (red vs. blue arrows) serves a fundamental role, as it effectively separates the process of inactivation during the action potential, controlled by the $\mathrm{O}$ - I transition, from the recovery from inactivation during the interspike interval, separately controlled by the I - C transitions.

As a result, inactivation from the open state proceeds very quickly, giving the channel just enough time to flow current and sufficiently depolarize the membrane and activate other voltage-gated ion channels. In contrast, recovery out of inactivation proceeds more slowly from the closed states, at a rate that determines a refractory period compatible with the maximal spiking rate of the neuron. Furthermore, bypassing the open state minimizes the flow of $\mathrm{Na}^{+}$ions in the wake of the action potential and thus economizes the energy utilized by cellular ionic pumps (Carter and Bean, 2009). A conceptual kinetic mechanism that adequately captures all these properties is shown in Fig. 5.1D (Kuo and Bean, 1994). 


\subsubsection{Nav channels have long-term inactivation}

Fast voltage-dependent activation (sub-millisecond) and inactivation (millisecond), as well as relatively fast recovery from inactivation (milliseconds) are kinetic properties common to all Nav channel subtypes, as described since the pioneering work of Hodgkin and Huxley (Hodgkin and Huxley, 1952). However, the Nav kinetic inventory is richer than that, including such behavior as "persistence" (French et al., 1990; Crill, 1996) or "resurgence" (Raman and Bean, 1997; Raman and Bean, 2001). In raphé and other neurons, Nav channels exhibit yet another interesting property: as illustrated in Fig. 5.2A, when subjected to brief, repetitive depolarizations that mimic trains of action potentials, the $\mathrm{Na}^{+}$current evoked by each pulse progressively diminishes to levels inversely proportional to the pulse repetition rate.

Furthermore, we can identify not one but two exponential components in the time course of recovery from inactivation, as shown in Fig. 5.2B. Following a brief depolarizing pulse that completely inactivates the channels, approximately $80 \%$ of the initially available current recovers fast (milliseconds), with a voltage-dependent time constant, whereas the remaining $20 \%$ recovers slowly (hundreds of ms), also with a voltagedependent time constant. In neonatal ventral raphé neurons, at $-80 \mathrm{mV}$, the fast and slow components have time constants $\tau$ and relative amplitudes $a$ of (mean $\pm \mathrm{SE}$ ): $\tau_{\text {fast }}=3.142$ $\pm 0.126 \mathrm{~ms}, \mathrm{a}_{\text {fast }}=0.786 \pm 0.013, \tau_{\text {slow }}=612 \pm 76 \mathrm{~ms}$, and $\mathrm{a}_{\text {slow }}=0.212 \pm 0.007(\mathrm{n}=18)$. At $-100 \mathrm{mV}, \tau_{\text {slow }}=209 \pm 39 \mathrm{~ms}$ and $\mathrm{a}_{\text {slow }}=0.199 \pm 0.01(\mathrm{n}=6)$. These values are very similar in mature dorsal raphé neurons: at $-100 \mathrm{mV}, \tau_{\text {slow }}=247 \pm 84 \mathrm{~ms}$ and $\mathrm{a}_{\text {slow }}=0.252$ \pm 0.033 ( $\mathrm{n}=5$ ). It is surprising that a brief $5 \mathrm{~ms}$ depolarizing pulse is sufficient to induce 
complete current inactivation but seconds are then necessary to completely remove inactivation, even considering the significant difference between the membrane potentials where activation/inactivation and recovery from inactivation occur $(-65 \mathrm{mV}$ in this case, from -15 to $-80 \mathrm{mV})$.

Similar Nav properties (i.e., adapting response to pulse trains and partial slow recovery from inactivation) have been observed in other neuronal types, such as hippocampal pyramidal neurons (Mickus et al., 1999). Appropriately, this phenomenology has been termed "prolonged inactivation" (Jung et al., 1997) or "long-term inactivation" (LTI) (Dover et al., 2010), to distinguish it from slow inactivation, a process whereby Nav channels slowly (hundreds of milliseconds to seconds, or more) become unavailable when held at depolarizing potentials, and also slowly return to full availability at hyperpolarizing potentials (Ruff et al., 1988; Fleidervish et al., 1996). In contrast, LTI represents fast entry into a long-lived inactivated state, from which recovery is very slow. Interestingly, the entry and exit time constants differ by three orders of magnitude: milliseconds at depolarizing potentials and seconds during hyperpolarization (Mickus et al., 1999; Milescu et al., 2010b). As we discuss next, this fast-slow duality has important functional consequences.

\section{$\underline{\text { 5.3.3 Mechanistic consequences of long-term inactivation }}$}

Like other channel relatives, Nav channels interact with auxiliary subunits and factors to create new functional behaviors (Grieco et al., 2005; Aman et al., 2009; Ben-Johny et al., 2014). The molecular factors that are responsible for LTI in different neuronal types have been identified as the FHFs (Smallwood et al., 1996; Liu et al., 2003; Wittmack et al., 
2004; Lou et al., 2005; Rush et al., 2006; Goldfarb et al., 2007; Dover et al., 2010). Through constrained kinetic modeling (Navarro et al., 2018; Salari et al., 2018) of a comprehensive set of voltage clamp data recorded from raphé neurons, we previously formulated a kinetic mechanism that explains well all tested Nav kinetic properties, including LTI (Milescu et al., 2010b). We verified this model in live neurons, using realtime computation (dynamic clamp), confirming that a model-generated current that replaced the TTX-blocked endogenous $\mathrm{I}_{\mathrm{Na}}$ was able to generate action potentials of similar shape and frequency. Other studies in different neuronal types have arrived at conceptually similar models (Goldfarb et al., 2007; Menon et al., 2009).

The model that we proposed to explain LTI in raphé neurons is shown in Fig. 5.3A, where the basic kinetic scheme introduced in Fig. 5.1D has been augmented with a nonconducting state $\left(\mathrm{S}_{13}\right)$ connected to the open state $\left(\mathrm{O}_{6}\right)$. $\mathrm{S}_{13}$ is a long-lived state representing the open channel blocked by the auxiliary factor. The $\mathrm{O}_{6}-\mathrm{S}_{13}$ rate constants take values that, in the context of the intrinsic Nav kinetics, result in the observed LTI phenomenology, i.e., the fast-slow bi-exponential recovery at hyperpolarizing potentials, out of the complete inactivation induced by a brief depolarization. The $\mathrm{O}_{6}-\mathrm{S}_{13}$ transition competes with the normal inactivation process that corresponds to the $\mathrm{O}_{6}-\mathrm{I}_{12}$ transition, with interesting mechanistic consequences. A brief depolarizing pulse from -80 to $0 \mathrm{mV}$ (Fig. 5.3B) takes the channel rapidly through the sequence of closed states, as voltage sensors activate. Once it reaches the open state, the channel has now two distinct pathways to follow: to inactivate "normally" into the $\mathrm{I}_{12}$ state, or to long-term inactivate 
into the $S_{13}$ state. According to our experimental data (Fig. 5.2B), $\approx 80 \%$ of the channels "choose" the normal inactivation pathway and $\approx 20 \%$ the LTI.

The $I_{12}$ and $S_{13}$ occupancy probabilities rise very quickly, reaching their respective values of 0.8 and 0.2 in a couple of milliseconds, as indicated by the time course of $P_{\mathrm{I}}$ and $P_{\mathrm{S}}$ in Fig. 5.3B (lower panel). However, these are not the equilibrium values: if the channels are maintained at depolarizing potentials for seconds, $P_{\mathrm{S}}$ very slowly reaches a considerably higher value of $\approx 0.7$, whereas $P_{\text {I }}$ drops accordingly to $\approx 0.3$. Hence, entry into the $S_{13}$ state is a bi-exponential process, with one component fast enough to reach completion during a brief voltage pulse - or action potential - and the other very slow, requiring several seconds to equilibrate. Since prolonged depolarizations are less likely to occur, the fast component is obviously the more physiologically relevant one.

What happens after a brief depolarizing pulse, upon repolarization? As depicted in Fig. 5.3C, those channels residing in the normal inactivated state $I_{12}$ cycle relatively fast (5 10 milliseconds) back into the non-inactivated closed states (C), without visiting the open state. In contrast, channels residing in the LTI state $S_{13}$ recover slowly, in seconds, reaching the non-inactivated states through the open state. Therefore, the sum occupancy probability of all non-inactivated states $\left(\mathrm{C}\right.$ states plus $\mathrm{O}_{6}$ state; $P_{\mathrm{C}+\mathrm{O}}$ in Fig 5.3C) rises from 0 to $\approx 1$, on a bi-exponential time course. The fast component corresponds to recovery from the normal inactivation process $\left(P_{\mathrm{I}}\right)$, whereas the slow component represents recovery from the LTI state $\left(P_{\mathrm{S}}\right)$. This dual fast-slow process explains the observed bi-exponential recovery from inactivation of $\mathrm{I}_{\mathrm{Na}}$ in raphé serotonergic neurons 
(Fig. 5.2B). Interestingly, the open probability $\left(P_{\mathrm{O}}\right)$ remains close to zero, because recovery from the LTI state through the open state is stretched over a long time interval.

\subsubsection{Nav channels are a molecular leaky integrator}

We now arrive at our main idea, that Nav channels implement a molecular leaky integrator, through an interaction with auxiliary factors. Mathematically, a continuoustime leaky integrator is governed by the differential equation:

$$
\frac{d y(t)}{d t}=x(t)-y(t) / \tau_{\text {leak }}
$$

where $y$ is the output signal, $x$ is the input signal, $t$ is time, and $\tau_{\text {leak }}$ is the leak time constant. Leaky integration can be more easily understood in the discrete time domain, as a mathematical operation that recursively calculates an output signal $y$ from an input signal $x$, as follows:

$$
y_{t+\delta \mathrm{t}}=y_{t} \times e^{-\delta t / \tau_{\text {leak }}}+x_{t+\delta \mathrm{t}} \times \delta t
$$

where $\delta t$ is the sampling time between two measurements. When $\tau_{\text {leak }}$ is infinite (or $\tau_{\text {leak }}$ $>\delta t$ ), the above equation reduces to a simple integration (or summation), where $y_{\mathrm{t}+} \delta_{\mathrm{t}}=$ $y_{\mathrm{t}}+x_{\mathrm{t}+} \delta_{\mathrm{t}} \times \delta t$. If the input signal contains digital data (i.e., 0 or 1 ), the integrator becomes an event counter. When $\tau_{\text {leak }}$ is zero (or $\tau_{\text {leak }}<<\delta$ ), the output signal becomes a scaled copy of the input signal, where $y_{\mathrm{t}+} \delta_{\mathrm{t}}=x_{\mathrm{t}+} \delta_{\mathrm{t}} \times \delta t$. Otherwise, when $\tau_{\text {leak }}$ takes a finite value, the output signal at a given time point is first "leaked" (i.e., reduced) by a factor determined by the ratio $\delta t / \tau_{\text {leak }}$, then "integrated" (i.e., added) with the input signal at the next time point, to calculate the next output. 
How does leaky integration apply to Nav channels? First, we consider the membrane potential $\left(V_{\mathrm{m}}\right)$ to be the input signal, and the occupancy of the LTI state $\mathrm{S}_{13}\left(P_{\mathrm{S}}\right)$ to be the output signal. We further consider $V_{\mathrm{m}}$ to be "digital" and take only two states: "low" in the interspike interval and "high" during the action potential, with the "low" state meaning functionally zero input (nothing to integrate). Finally, we consider that the time scale of the leak process is orders of magnitude longer than the width of an action potential. Under these conditions, the integration step corresponds to quickly incrementing $P_{\mathrm{S}}$ whenever the input signal $V_{\mathrm{m}}$ switches to a high state (an action potential), as shown in Fig. 5.4A, and the leak step corresponds to slowly decrementing $P_{\mathrm{S}}$ whenever $V_{\mathrm{m}}$ switches to a low state (the inter-spike interval), as shown in Fig. 5.4B. As indicated by our experimental data (Fig. 5.2B) and correctly predicted by our model, the arrival of a brief depolarization, such as an action potential, prompts $\approx 20 \%$ of all available (non-inactivated) channels to rush into the long-term inactivated state $S_{13}$, whereas the other $\approx 80 \%$ quickly occupy the inactivated state $\mathrm{I}_{12}$, with time courses as shown in Fig. 5.3B. Channels that are already inactivated will maintain their state. Because $P_{\mathrm{S}}$ is in fact a probability bound by 1 , it cannot increase indefinitely. In fact, each action potential can only increase $P_{\mathrm{S}}$ by $\approx 20 \%$ of the fraction of available channels. At the beginning of a spike train, when all channels are available, this increment is $\approx 0.2$ but progressively gets smaller, as fewer channels remain available (Fig. 5.4C). The specific value of the increment depends on the relative kinetics of $\mathrm{O}_{6}-\mathrm{S}_{13}$ and $\mathrm{O}_{6}-\mathrm{I}_{12}$ transitions. Importantly, as predicted by the time course of $P_{\mathrm{S}}$ in Fig. 5.3B, changing the width of the action potential would not significantly alter the $20 / 80 \%$ ratio, unless the 
width were shorter than $\approx 1 \mathrm{~ms}$, or if the depolarization were extended to hundreds of milliseconds or seconds, in which case $P_{\mathrm{S}}$ would eventually reach an equilibrium value of $\approx 0.7\left(P_{S}^{\infty}\right.$ in Fig. 5.3B $)$.

Once the action potential ends and $V_{\mathrm{m}}$ switches to the low state, $P_{\mathrm{S}}$ starts to "leak", because the input signal is "zero" in the interspike interval and there is nothing to "integrate". Thus, $P_{\mathrm{S}}$ decays exponentially, with the same time constant as the slow

component of recovery from inactivation (hundreds of milliseconds). Those channels that were long-term inactivated (occupying the $S_{13}$ state) at the beginning of the interspike interval will remain unavailable to conduct current for an accordingly long time interval, on the order of seconds. In contrast, those channels that were normally inactivated (occupying the $\mathrm{I}_{12}$ state) will become available much sooner, after only a few milliseconds. If another action potential were triggered while some channels were still residing in either the long-term inactivated state $S_{13}$ or in the inactivated state $I_{12}$, the available (non-inactivated) channels will again divide $80 / 20 \%$ between normal inactivation and long-term inactivation, and so on. As a result, the occupancy of the $S_{13}$ state would keep increasing with each action potential (by progressively smaller increments), unless the interspike intervals were long enough to allow complete recovery out of the $S_{13}$ state.

\section{$\underline{\text { 5.3.5 Nav channels detect action potentials and measure spiking frequency }}$}

The use of an integrator is obvious - to summate, to count - but what about a "leaky" integrator? As it happens, a leaky integrator not only describes many real-world phenomena, such as rain accumulating into a lake that drains into a river, but also has 
many technical applications. One of the most obvious is to convert the frequency of an input signal into the amplitude of an output signal. In our own electrophysiology backyard, it is customary to pass a nerve signal through a leaky integrator (implemented in hardware or software) to convert noisy and dense spike trains into an amplitude signal that more legibly indicates the frequency of those spikes.

As a molecular leaky integrator, Nav channels can also "measure" the frequency of action potentials and "store" it in the occupancy of the LTI state $S_{13}$. As demonstrated by the experimental data shown in Fig. 5.2A, the amount of $\mathrm{Na}^{+}$current evoked with $5 \mathrm{~ms}$ depolarizing pulse trains decays exponentially with each pulse, and decays more at higher repetition rates. In Fig. 5.4C, we calculate and plot $P_{\mathrm{S}}$ using the equation $P_{\mathrm{S}}=1-\mathrm{I}_{\mathrm{Na}} /$ $\mathrm{I}_{\mathrm{Na}}{ }^{0}$, where $\mathrm{I}_{\mathrm{Na}}$ is the peak $\mathrm{Na}^{+}$current measured for a given pulse and $\mathrm{I}_{\mathrm{Na}}{ }^{0}$ is the peak $\mathrm{Na}^{+}$ current for the first pulse in the series. The rationale of using this equation to obtain $P_{\mathrm{S}}$ is that the inter-pulse interval $(195,95$, and $45 \mathrm{~ms}$ in this case) is long enough to allow full recovery from the normal inactivation, whereas recovery from the LTI state will be incomplete, as we know from the data shown in Fig. 5.2B. Thus, having a fraction of channels still trapped in the LTI state will proportionally reduce the maximal $\mathrm{I}_{\mathrm{Na}}$. As seen for this representative data set, $P_{\mathrm{S}}$ reaches steady values of $\approx 0.3$ at $5 \mathrm{~Hz}, \approx 0.4$ at $10 \mathrm{~Hz}$, and $\approx 0.5$ at $20 \mathrm{~Hz}$, exhibiting a nonlinear dependence on the pulse repetition rate.

To better understand how Nav channels may interpret action potential frequency, we simulated the response of our Nav model to a train of $5 \mathrm{~ms}$ depolarizing pulses from -80 to $0 \mathrm{mV}$, repeated at different rates, and compared it with the response of a mathematical leaky integrator. For the sake of simplicity, the mathematical leaky integrator was 
presented with the same train of pulses as the Nav model, but the input variable $x$ was assigned 0 and 1 values, instead of -80 and 0 , respectively. To match the behavior of the Nav model, we also modified Eq. 2, as follows:

$$
\begin{aligned}
& y_{t+\delta \mathrm{t}}=y_{t} \times e^{-\delta t / \tau_{\text {leak }}}+x_{t+\delta \mathrm{t}} \times f_{t+\delta \mathrm{t}}, \\
& f_{t+\delta \mathrm{t}}=y_{\text {inc }} \times\left(\delta t / t_{P}\right) \times\left(1-y_{t} \times e^{-\delta t / \tau_{\text {leak }}}\right),
\end{aligned}
$$

where $y_{\text {inc }}=0.2$ represents the $20 \%$ maximum increase in the output variable $y$, spread over the duration $t_{\mathrm{P}}$ of a pulse. Thus, $f$ is an ad hoc expression that ensures that, during a pulse in the input variable $x$, the output variable $y$ can only increase by $20 \%$ out of the difference to 1 (i.e., $1-y_{t} \times e^{-\delta t / \tau_{\text {leak }}}$ ), in the same way as $P_{\mathrm{S}}$ can only increase by $20 \%$ of the fraction of available channels (i.e., $1-P_{\mathrm{S}}$ ). As a result, the range of the output variable $y$ is restricted between 0 and 1 , similarly to $P_{\mathrm{S}}$.

Our Nav model has a leak time constant $\tau_{\text {leak }} \approx 435 \mathrm{~ms}$ at $-80 \mathrm{mV}$, a normal inactivation / LTI ratio of $80 / 20 \%$, and an equilibrium $S$ state occupancy $P_{S}^{\infty} \approx 0.7$. These quantities depend on the specific values and voltage dependence of the $\mathrm{O}_{6}-\mathrm{S}_{13}$ rate constants $\left(k_{6,13}\right.$ $=400.8 \times \mathrm{e}^{-0.011 \times \mathrm{V}}$ and $\left.k_{13,6}=0.207 \times \mathrm{e}^{-0.031 \times \mathrm{V}}\right)$, in the context of all the other rate constants. Keeping the exponential factors $k_{6,13}^{1}$ and $k_{13,6}^{1}$ and all other rate constants unchanged, $\tau_{\text {leak }}$ depends mostly on the pre-exponential factor $k_{13,6}^{0}$ (lower value increases $\tau_{\text {leak }}$ ), the normal inactivation/LTI ratio depends on the pre-exponential factor $k_{6,13}^{0}$ (lower value increases the ratio), and $P_{S}^{\infty}$ depends on the ratio between $k_{6,13}^{0}$ and $k_{13,6}^{0}$ (lower ratio decreases $\left.P_{S}^{\infty}\right)$ 
As indicated by the red trace in Fig. 5.4D, when the Nav model is presented with a train of depolarizing pulses, $P_{\mathrm{S}}$ reaches levels that depend nonlinearly on the pulse repetition rate. At any given pulse frequency, $P_{\mathrm{S}}$ oscillates between a maximum reached at the end of a pulse, and a minimum reached at the end of the interpulse interval. At the maximum tested frequency of $100 \mathrm{~Hz}, P_{\mathrm{S}}$ oscillates between 0.92 and 0.93 . Then, under constant stimulus, $P_{\mathrm{S}}$ decays down to its equilibrium value $P_{S}^{\infty}$ (see Fig. 5.3B). For comparison, we modified the $\mathrm{O}_{6}-\mathrm{S}_{13}$ rate constants $\left(k_{6,13}=205.7 \times \mathrm{e}^{-0.011 \times \mathrm{V}}\right.$ and $\left.k_{13,6}=0.106 \times \mathrm{e}^{-0.031 \times \mathrm{V}}\right)$ to obtain $\tau_{\text {leak }} \approx 820 \mathrm{~ms}$ and $90 / 10 \%$ normal inactivation / LTI ratio, while keeping the same $P_{S}^{\infty}$. As indicated by the blue trace in Fig. 5.4D, in this case $P_{S}$ exhibits reduced oscillations and takes longer to reach steady state at a given pulse rate but follows the same overall trend with the increase in stimulus frequency. Finally, the mathematical leaky integrator responds in a similar fashion, although it starts to deviate at higher frequencies and under continuous input, where it reaches a maximum, in contrast to $P_{\mathrm{s}}$, which slowly decays to $P_{S}^{\infty}$.

\subsubsection{Nav channels drive spiking frequency}

As demonstrated in Fig. 5.4C and D, Nav channels respond to stimulation frequency by changing the occupancy of the long-term inactivated state, which, in turn, changes the amount of available $\mathrm{I}_{\mathrm{Na}}$. This observation raises the reciprocal question: does a raphé neuron respond to the amount of available $\mathrm{I}_{\mathrm{Na}}$ by changing its spiking frequency? $\mathrm{I}_{\mathrm{Na}}$ can potentially drive spiking frequency via two interrelated mechanisms: directly, by controlling the rate of depolarization in the interspike interval, and indirectly, by shaping the action potential waveform and thus affecting the other ionic currents that flow during 
the action potential depolarization and during the ensuing interspike interval. To test this idea, we used dynamic clamp to inject a model-based $\mathrm{I}_{\mathrm{Na}}$ in ventral raphé neurons, and measured the response of the cell to increasing levels of Nav conductance $\left(G_{\mathrm{Na}}\right)$.

Indeed, raphé neurons do respond to the level of available $\mathrm{I}_{\mathrm{Na}}$ by changing their spiking frequency, as shown in Fig. 5.5A. The frequency vs. $\mathrm{G}_{\mathrm{Na}}$ response is approximately linear over a range of $\mathrm{G}_{\mathrm{Na}}=4$ to $16 \mathrm{nS} / \mathrm{pF}$, although it starts to show saturation at higher levels $(25 \mathrm{nS} / \mathrm{pF})$ (Fig. 5.5B). The effect of $\mathrm{I}_{\mathrm{Na}}$ on spiking frequency is clarified in Fig. 5.5C, where three example waveforms, each containing one action potential and the subsequent interspike interval obtained under a different $\mathrm{G}_{\mathrm{Na}}$, are shown aligned. As can be observed, the rate of membrane depolarization in the interspike interval is virtually $\mathrm{I}_{\mathrm{Na}^{-}}$and frequency-independent immediately after an action potential, up to $\approx-53 \mathrm{mV}$. From this point, the rate of depolarization changes proportionally to $\mathrm{G}_{\mathrm{Na}}$, determining the duration of the interspike interval. Thus, at low firing frequency, Nav channels spend more time above $-55 \mathrm{mV}$, which causes more closed-state inactivation and explains why the $\mathrm{I}_{\mathrm{Na}}$ is disproportionately smaller. The amount of available $\mathrm{I}_{\mathrm{Na}}$ also changes the peak membrane potential reached during an action potential.

\subsubsection{Nav channels are a molecular controller that regulates neuronal firing}

\section{$\underline{\text { rate }}$}

The experiments described in Figs. 5.2 and $\mathbf{5 . 5}$ clearly demonstrate that not only can Nav channels sense the frequency of action potentials, but they also can drive the spiking rate in raphé neurons. Thus, Nav channels implement a negative feedback loop that can 
regulate the firing frequency of the host neuron, as shown in Fig. 5.6A. Conceptually, they are similar to a process controller used in engineering applications, as illustrated in Fig. 5.6B. As a technological example, one could take the home furnace as the "process", the indoor temperature as the "process variable", and human comfort as the "product". A sensor measures the temperature and forwards the measurement to a thermostat ("controller"), which calculates the difference ("error") between a user-prescribed value ("set point") and the measured value. A control algorithm processes the error and determines the timing and the amount of gas to burn in the furnace ("control variable") such as to maintain the actual indoor temperature relatively constant and approximately equal to the desired temperature. The furnace will burn gas when the error is positive, which will increase the temperature and eventually make the error zero or negative, which in turn will instruct the furnace to stop burning gas, until the temperature drops again and the error becomes again positive.

The similarity between the Nav-based controller and the technological controller is quite striking, although not perfect. In the neuronal case (Fig. 5.6A), we can identify the "process" as the neuron, the "process variable" as the spiking frequency, and the "product" as serotonin. The Nav kinetic mechanism embodies the "sensor", the "set point", and the "controller" altogether: $i$ ) spiking frequency is measured by the occupancy of the LTI state $\mathrm{S}_{13}$, ii) the set point is determined by the rate constants of the $\mathrm{O}_{6}-\mathrm{S}_{13}$ transition, and iii) the control algorithm is simply represented by the mutually exclusive relationship between the occupancy of the LTI state and the fraction of Nav channels available to generate current, which is equal to $1-P_{\mathrm{S}}$. Finally, the "control variable" is 
represented by the amount of available $\mathrm{I}_{\mathrm{Na}}$, which can drive spiking frequency and thus closes the loop.

\subsection{Discussion}

Nav channels play a fundamental role in cellular excitability, acting as a nonlinear amplifier that converts a small membrane depolarization into an action potential. Their intrinsically complex kinetic mechanism (Armstrong and Gilly, 1979; Armstrong, 2006) is further tweaked in different neuronal populations, to make Nav channels drive spontaneous spiking (Do and Bean, 2003), enable fast spiking (Raman and Bean, 2001; Khaliq et al., 2003), or establish complex firing modes (Magistretti et al., 2006; Yamanishi et al., 2018). Here, we showed that adding a state of long-term inactivation (Fig. 5.3A), as can be created by an interaction with auxiliary factors (Dover et al., 2010), significantly expands the Nav computational repertoire. LTI effectively turns the Nav channel into a molecular leaky integrator that can analyze the firing activity of the host neuron and encode its spiking frequency into the fraction of available sodium current (Fig. 5.4). In cells where spiking frequency can be modulated by the available Nav conductance, such as the serotonergic raphé neurons (Fig. 5.5), this frequency-toavailable current conversion can establish a negative feedback control loop that can regulate the frequency of action potentials (Fig. 5.6).

Mathematically, a leaky integrator summates over time an input signal into an output signal, but the output steadily decays (Eq. 2). Studying for an exam is a good analogy: we quickly accumulate knowledge with every page we read, while at the same time we slowly forget whatever we learned. In the field of electrical engineering, a simple leaky 
integrator can be implemented with an RC circuit. In the molecular world, nature has found a simple and elegant way of converting the Nav channel into a leaky integrator, while preserving its basic functionality as a spike generator - and partial pacemaking driver in raphé neurons. LTI is a mechanism that renders a fraction of the total number of Nav channels functionally non-available, in the sense that these channels are trapped in a non-conducting state and thus cannot contribute current to an action potential. This fraction increases and decreases with dynamics dictated by the frequency of action potentials, effectively becoming a measure of neuronal activity. For this mechanism to work, a single action potential must quickly increment the non-available fraction, whereas the subsequent interspike interval must slowly decrement it. In the case of LTI in raphé neurons, the two time constants are separated by three orders of magnitude (Fig.

\subsection{B and $\mathbf{C})$.}

The simplest kinetic mechanism compatible with the observed LTI behavior is shown in Fig. 5.3A, where an additional non-conducting state is connected to the open state of the basic Nav mechanism shown in Fig. 5.1D (Dover et al., 2010; Milescu et al., 2010b). This model predicts the critically important large discrepancy between LTI entry and exit time constants, yet realistically requires only minimal charge for the LTI transition. Nevertheless, the LTI mechanism - as well as the Nav mechanism itself - may be different and possibly more complex in reality, and needs further investigation. More work is also necessary to identify the specific subtypes of Nav channels and FHFs that are functional in both ventral and dorsal raphé neurons. 
Although we examined here its computational aspects, the specific function of the Nav LTI regulatory mechanism in raphé neurons remains to be investigated in full. What would happen in its absence? Clearly, for the same level of Nav channel expression, there would be more sodium current available and, considering the results shown in Fig 5.5, the firing frequency would be greater. Thus, one could speculate that one role of LTI is to make spiking frequency less dependent on Nav channel expression. Of course, as demonstrated in Fig. 5.5, frequency still increases with $\mathrm{G}_{\mathrm{Na}}$, but this increase would be steeper without LTI. If this hypothesis were true, it would be interesting to know how many neuronal types rely on this mechanism.

Another possibility is that LTI is one of several mechanisms that force raphé neurons to fire at relatively low frequency. In a previous study (Milescu et al., 2010b), we showed that ventral raphé neurons react to a depolarizing current injection with a proportional increase in spiking frequency, but they quickly adapt and then slowly return to a low frequency. In part, this adaptation depends on Nav channel long-term inactivation. Interestingly, the same type of kinetic model with open state block can account for the resurgent sodium current (Raman and Bean, 2001). However, in that case the entry and exit time constants have comparable values. That model can also be considered a leaky integrator but the fraction of channels that enter the blocked state during a brief depolarization becomes available immediately upon repolarization and thus augments the subthreshold depolarizing $\mathrm{Na}^{+}$current, helping the hosting neuron to spike faster. In contrast, LTI may play the opposite role, by decreasing the amount of $\mathrm{Na}^{+}$current (both sub- and suprathreshold) and making it harder for the neuron to spike at high frequency. 
We are not aware of other studies where Nav channels - or other channels - have been identified as molecular leaky integrators, even though the leaky integrator is a powerful concept that has long been associated with neural computation, from individual neurons and circuits to cognitive processes (Knight, 1972; Cook and Maunsell, 2002; Mitani et al., 2013; Portugues et al., 2015; Groschner et al., 2018). In excitable cells, a molecular leaky integrator can be a tool for monitoring and regulating cellular activity, as we showed here for serotonergic raphé neurons. In fact, the intracellular $\mathrm{Ca}^{2+}$ also acts as a leaky integrator, quickly incrementing its concentration with each action potential, via $\mathrm{Ca}^{2+}$ influx through voltage-gated calcium channels, and more slowly decrementing it during periods of quiescence (Gorman and Thomas, 1978; Helmchen et al., 1996). Thus, the envelope of intracellular $\mathrm{Ca}^{2+}$ concentration becomes a measure of cellular activity that can be used to regulate a variety of $\mathrm{Ca}^{2+}$-dependent cellular processes (Gardos, 1958; Yuste et al., 2000; O'Leary et al., 2013), including the activity of many types of ion channels (Meech and Standen, 1975; Keen et al., 1999; Peterson et al., 1999; Deschênes et al., 2002; Wen and Levitan, 2002; Hartzell et al., 2005), which in turn can change the firing activity. As a leaky integrator established by the long-term inactivation process, Nav channels add another powerful mechanism for sensing and regulating the activity of excitable cells. 


\subsection{Figures and figure legends}

A

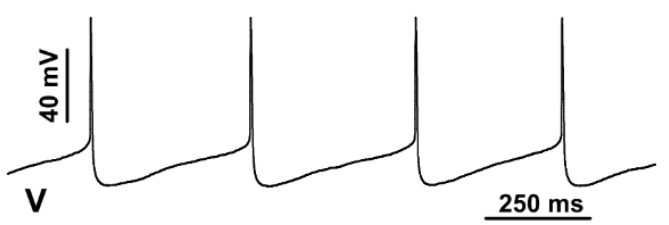

B

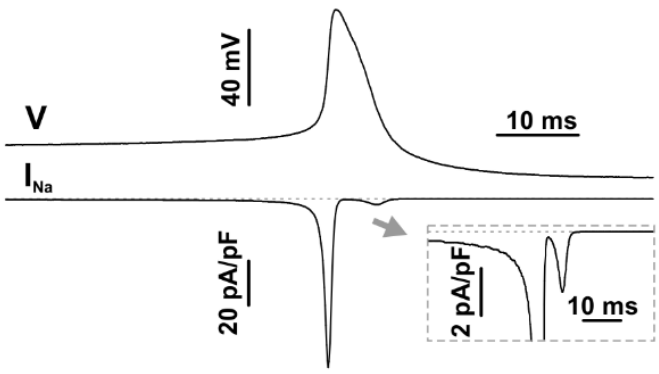

C

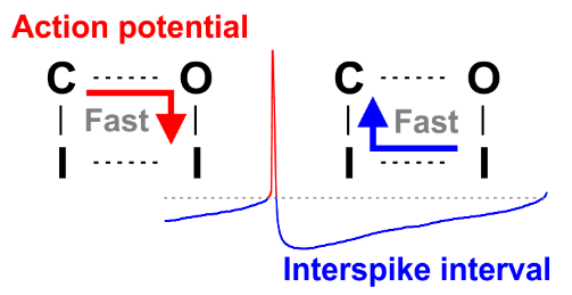

D

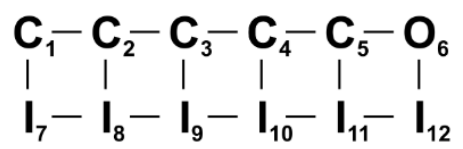

Figure 5.1. Spontaneous firing in serotonergic raphé neurons and the contribution of Nav channels. A and B) Raphé neurons are characterized by slow and regular spiking and broad action potentials, with the spike-generating sodium current $\left(\mathrm{I}_{\mathrm{Na}}\right)$ mostly restricted to the depolarization phase. C) Schematic of state transitions undertaken by Nav channels during the spiking cycle ("C" - closed, "O" - open, "I" - inactivated states). D) Conceptual Nav state model proposed to explain the fundamental kinetic properties of $\mathrm{I}_{\mathrm{Na}}$ in mammalian central neurons (Kuo and Bean, 1994). The representative current clamp recordings in $\mathbf{A}$ and $\mathbf{B}$ were obtained in neonatal rat brainstem slice preparations. In $\mathbf{B}, \mathrm{I}_{\mathrm{Na}}$ was calculated in real-time and injected in the cell using dynamic clamp, as previously reported (Milescu et al., 2010b). 


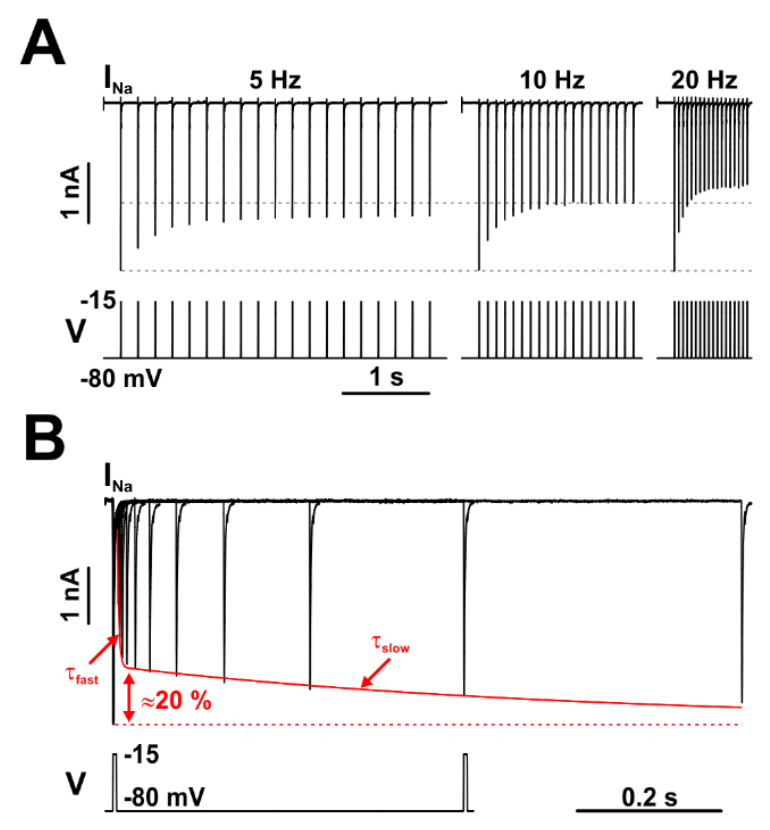

Figure 5.2. Nav channels raphé neurons exhibit a slow kinetic component. A) The fraction of Nav channels available to generate current decays exponentially, when tested with trains of brief depolarizing voltage pulses ( $5 \mathrm{~ms}$ at $-15 \mathrm{mV}$, repeated at 5,10 , or $20 \mathrm{~Hz}$ ). The decay is greater at higher repetition rates. Each pulse completely inactivates $\mathrm{I}_{\mathrm{Na}}$, which then partially recovers from inactivation in the subsequent hyperpolarizing interval, at $-80 \mathrm{mV}$. B) The timing of recovery from inactivation was tested with a two-pulse protocol, where the first pulse (5 ms at $-15 \mathrm{mV})$ inactivates the channels and the second pulse tests availability versus time at $-80 \mathrm{mV}$. As indicated by the two time constants $\left(\tau_{\text {fast }}\right.$ and $\left.\tau_{\text {slow }}\right)$, recovery from inactivation is a bi-exponential process, with the slow component accounting for approximately $20 \%$ of the total current. The representative voltage clamp recordings in $\mathbf{A}$ and $\mathbf{B}$ were obtained in neonatal rat brainstem slice preparations. 


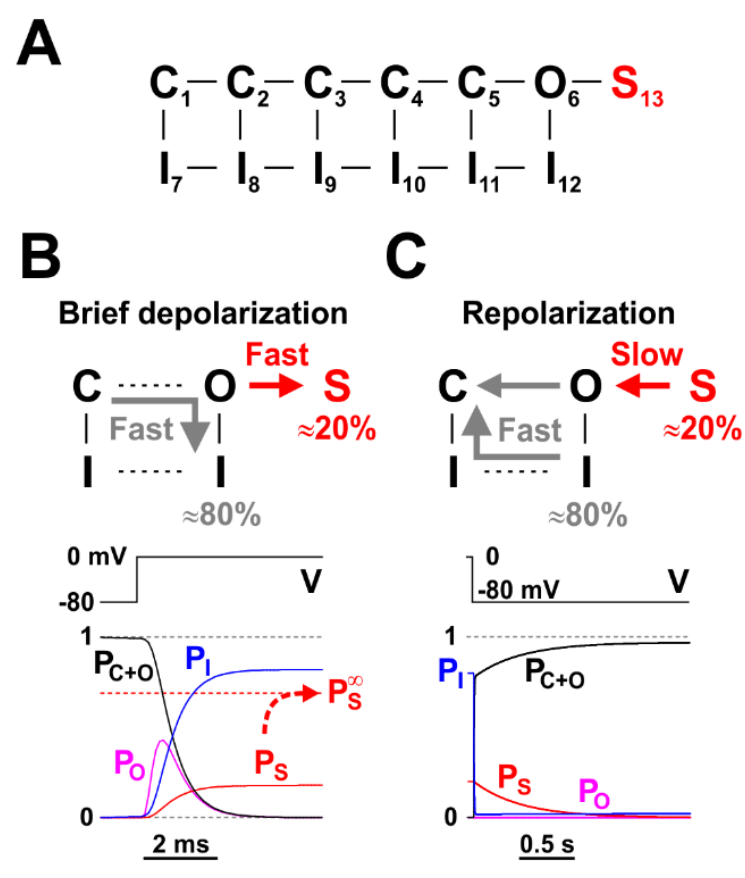

Figure 5.3. Nav long-term inactivation. A) Conceptual Nav state model that adds one nonconducting state $\left(S_{13}\right)$ to the model shown in Fig. 5.1D, to explain the slow kinetic component illustrated in Fig. 5.2. $S_{13}$ is a state of long-term inactivation. $\mathbf{B}$ and $\left.\mathbf{C}\right)$ State transitions undertaken by the channel during a brief depolarization $(\mathbf{B})$ and in the subsequent hyperpolarizing interval $(\mathbf{C})$. During depolarization, $\approx 80 \%$ of channels follow the standard C-O-I pathway, whereas the remaining $\approx 20 \%$ enter the $\mathrm{S}$ state. The O-I and O-S transitions are both fast, as illustrated in the bottom left panel, and compete with each other. When the membrane potential returns to more negative values, the fraction of channels in the I states recovers quickly, whereas the $\mathrm{S}$ state fraction recovers slowly, explaining the bi-exponential recovery from inactivation illustrated in Fig. 5.2B. $P_{C+O}, P_{O}, P_{I}$, and $P_{S}$ represent occupancies of closed and open, open, inactivated, and long-term inactivated states, respectively 


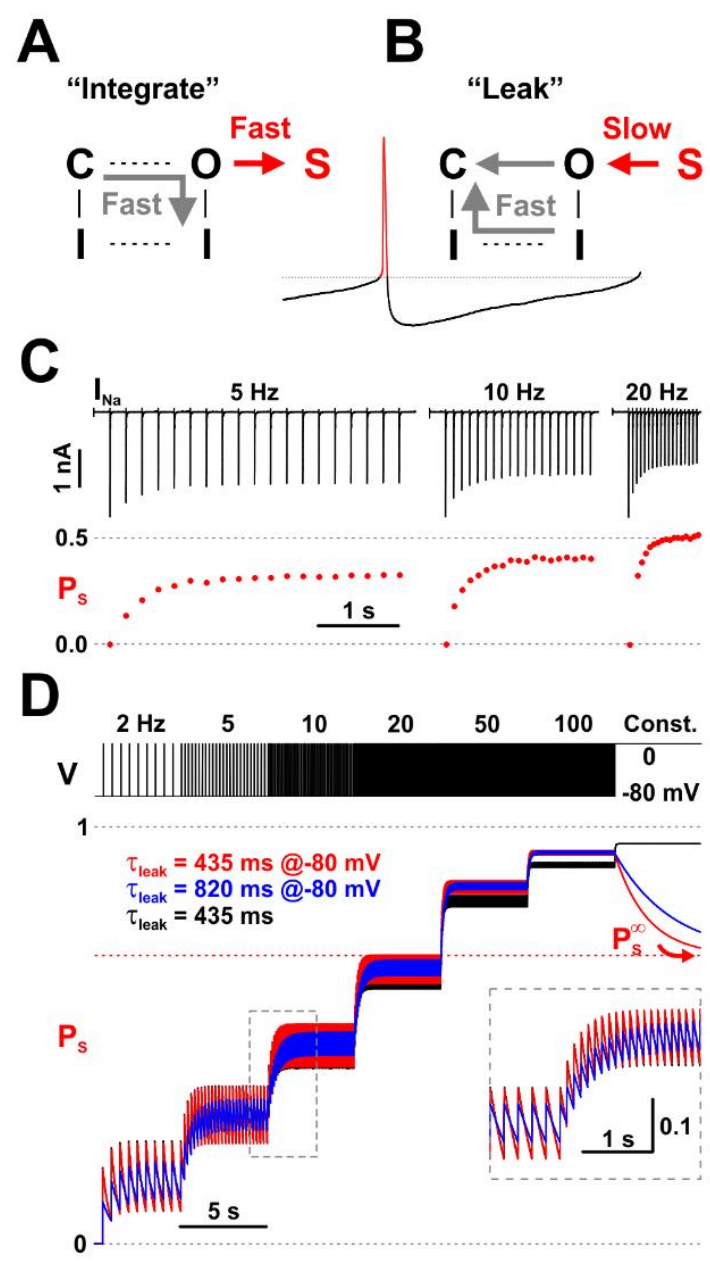

Figure 5.4. Nav channels implement a molecular leaky integrator that measures spiking frequency. A) The "integration" is represented by the quick entry of channels into the LTI state $\mathrm{S}$, during an action potential. B) The "leak" is the slow transition out of the S state, during the interspike interval. C) The average occupancy of the LTI state $\left(P_{\mathrm{S}}\right)$ increases with pulse repetition rate. The current trace is as in Fig. 5.2A. D, Testing the leaky integrator with trains of brief voltage pulses $(5 \mathrm{~ms}$ at $0 \mathrm{mV}$, from $-80 \mathrm{mV}$ ), with different repetition rates (2 to $100 \mathrm{~Hz}$ ) or at constant depolarization. The average occupancy of the $\mathrm{S}$ state is a function of pulse frequency. The Nav model in Fig. 5.3A was tested with two sets of kinetic parameters for the $\mathrm{O}_{6}-\mathrm{S}_{13}$ transition, corresponding to $\tau_{\text {leak }}=435 \mathrm{~ms}$ and $80 / 20 \%$ normal inactivation vs. LTI ratio (red trace; $k_{6,13}=400.8 \times \mathrm{e}^{-0.011 \times \mathrm{V}}$ and $k_{13,6}=0.207 \times \mathrm{e}^{-0.031 \times \mathrm{V}}$ ) or $820 \mathrm{~ms}$ and $90 / 10 \%$ ratio (blue trace; $k_{6,13}=205.7 \times \mathrm{e}^{-0.011 \times \mathrm{V}}$ and $\left.k_{13,6}=0.106 \times \mathrm{e}^{-0.031 \times \mathrm{V}}\right)$. Both sets have $P_{S}^{\infty} \approx 0.7$. For comparison, the response of a discrete time mathematical leaky integrator with $\tau_{\text {leak }}=435 \mathrm{~ms}$ (black trace; Eq. 3). 


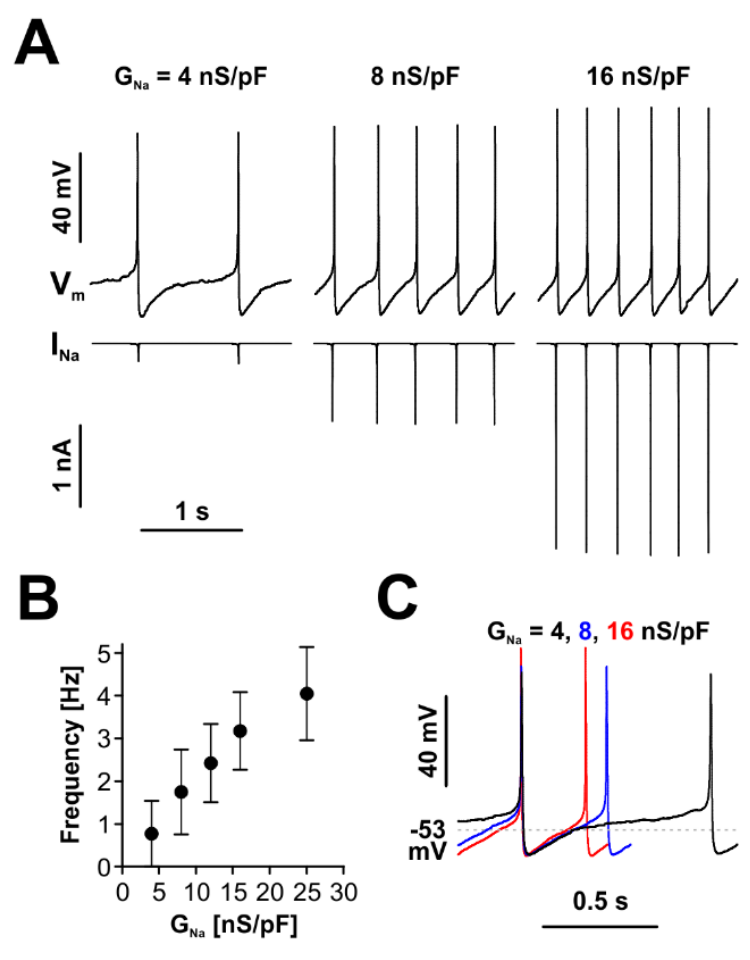

Figure 5.5. Nav channels drive spiking frequency in serotonergic raphé neurons. A) Representative dynamic clamp recordings, where a model-based $\mathrm{I}_{\mathrm{Na}}$ was injected in a neuron, under different levels of Nav conductance $\left(\mathrm{G}_{\mathrm{Na}}\right)$. The endogenous sodium current was blocked with bath-applied TTX. B) Spiking frequency increases proportionally with $\mathrm{G}_{\mathrm{Na}}$, over a range typical for neonatal ventral raphé neurons. C) The rate of membrane depolarization in the interspike interval is virtually $\mathrm{I}_{\mathrm{Na}}$-independent immediately after the action potential, up to $\approx-53$ $\mathrm{mV}$, after which it changes proportionally to $\mathrm{G}_{\mathrm{Na}}$. 
A

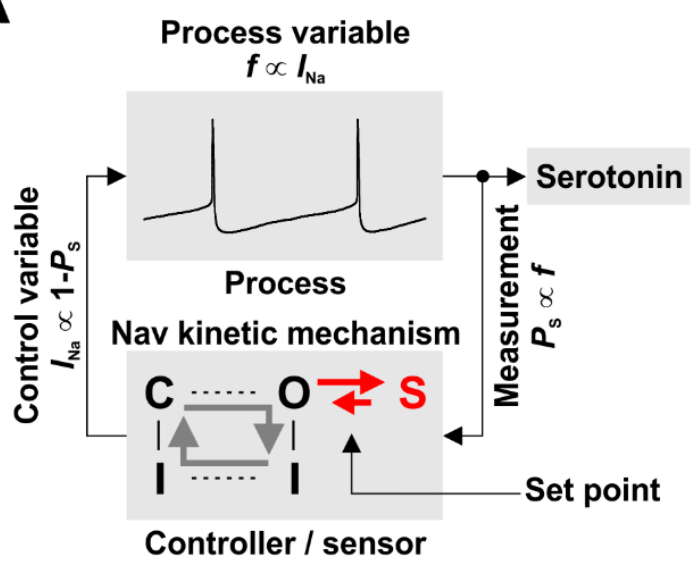

B

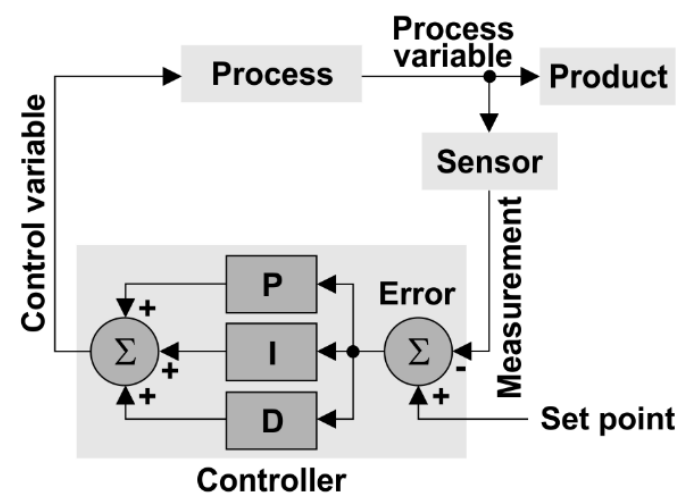

Figure 5.6. Nav channels can regulate spiking frequency in serotonergic raphé neurons in a negative feedback loop. A) Spiking frequency $f$ is "measured" by the occupancy of the longterm inactivated state $\left(\mathrm{P}_{\mathrm{S}}\right)$. For example, an increase in $f$ causes an increase in $\mathrm{P}_{\mathrm{S}}$, which determines a decrease in the fraction of Nav channels available to generate current, equal to 1 $\mathrm{P}_{\mathrm{S}}$. In turn, this decrease in $\mathrm{I}_{\mathrm{Na}}$ reduces firing frequency, closing the loop. The kinetics of the long-term inactivation establish the operating point of the control loop. B) For comparison, a conceptual schematic of controllers used in engineering applications. "P" stands for proportional, "I" for integral, and "D" for derivative error correction. 


\section{Chapter 6}

\section{Concluding remarks}

In order for organisms to produce behaviors, organized networks of neurons must be able to integrate sensory information and relay an appropriate response. To perform these functions, neurons are arranged in networks that contain pacemaking neurons, some that set the pace of bursting output (PreBötzinger bursters (Smith et al., 1991), others tonically fire and respond to physiological variables (raphé neurons (Ptak et al., 2009), excitatory interneurons that follow the pacemakers and help generate the rhythm (Koizumi et al., 2013), inhibitory interneurons that help with coordinated output between networks (Koizumi et al., 2013), and motor neurons that produce the final output synapsing on individual muscle fibers to produce contractions. The system (body/nervous system) as a whole is complex and works to maintain overall homeostasis.

This work aimed to provide new ideas in the field of neuroscience, to improve electrophysiology experience and to develop new methods for understanding how ion channels contribute to neuronal activity. Biology is complex and one must narrow focus to identify questions or problems that can be addressed with one's toolbox and, when needed, expand the toolbox. To keep things simple, I targeted sets of intrinsic pacemaker neurons to take advantage of their ability to maintain activity under synaptic blockade to isolate individual neuron activity from network activity. Removing network activity results in cleaner recordings but also simplifies the computation of what is driving the cell to fire. Although we try to isolate a single mechanism, pacemakers that drive 
essential behaviors such as cardio-respiratory function, have multiple, redundant mechanisms to ensure proper function.

Trying to understand ion channel kinetic mechanisms by developing Markov state models requires good quality data sets, obtained with many voltage protocols, to be able to identify different functional states. Single channel recordings can be used to predict the number of states through the distributions of the open probability histogram. The challenge with interpreting ion channel data lies with the concept of hidden states, such as inactivation. Our observations in electrophysiology recordings are useful for identifying the open (conducting) state of a channel. Programs such as QuB were developed to quickly calculate the linear algebra required to determine the $\mathbf{Q}$ matrix (transition rate matrix) and fit the free parameters of the model to the data (Qin, 1996, 2000a; Milescu, 2005). To identify other protein conformations, we use voltage clamp pulses to trace the rise and decay from thousands of individual channels. In the case of Nav channels, a brief $5 \mathrm{~ms}$ depolarizing pulse around $-20 \mathrm{mV}$ will trigger fast inward current (activation) that quickly turns off (inactivation) prior to the length of the pulse. A simple two state, Closed and Open, model cannot explain the time course of activation, which requires additional closed to closed transitions (Kienker, 1989; Milescu, 2005). As voltage-gated ion channels are tetrameric or pseudo-tetrameric proteins with functional domains, such as a voltage sensor that must move to open the pore (Horn et al., 2000; Chanda and Bezanilla, 2002a, b), structurally informed Markov models should take into consideration prior knowledge, including identifiable states such as activated voltage 
sensors, or identifiable state transitions, such as Nav channel inactivation from the closed state (Armstrong and Bezanilla, 1977; Bezanilla and Armstrong, 1977).

A long-standing issue in the ion channel field is how to develop models that describe new data, while at the same time accounting for results obtained in previous experiments. For example, existing known voltage-sensitivity values or scaling relationships between parameters are values determined for voltage-gated channels could be used as a starting place to fit a new data set. Other data, such as the open probability, can be reduced to a single value, yet the open probability is essentially determined by the entire model and thus depends in a complex fashion on the rate constants. Chapter 2 describes the mathematical methods and procedures used to implement linear parameter constrains and non-linear behavioral constraints that can be used to incorporate existing knowledge into the kinetic models of ion channels and other proteins. These methods are independent of the optimization method and can be applied to a variety of systems.

Applying kinetic constraints allows one to reduce the number of free parameters and thus reduce the parameter search space of the model. Ideally, one should use the most compact model that can best explain all the data, but one should also account for everything else that is known or hypothesized about the molecule of interest. Application of linear parameter constraints can reduce the number of free parameters by one for each constraint. Chapter 2 also introduced the use of slack variables that can be used to modify an inequality relationship into an equality to constrain rates to certain values, such as greater than 0 . Inequality linear constraints do not reduce the number of free parameters, but effectively reduce the parameter search space. 
Behavioral constraints were implemented by addition of a penalty parameter to the overall cost function, which increases if the output of the iteration produces a model that does not produce the desired behavior. These behaviors are still limited to functions that can be quickly calculated from the generated data, however the advantage to enforce a known single-channel open probability, while simultaneously fitting macroscopic data, substantially improves how models can explain the overall ion channel activity. Modeling remains a challenging process and requires patience, as one restarts the optimizer with different initial parameters values to avoid local minima. Constraining models allows one to reduce the number of free parameters and create more informed models, however each constraint changes the free parameter landscape, changing how the optimization engine explores the space, and requiring more time to explore. By applying behavioral constraints, one can nudge the cost function to produce more informed models, but the constraints must be compatible with the data.

Chapter 3 describes the computational tools used to develop a system that integrates a live camera feed with positional feedback of the sample prep. The microscope on my electrophysiology rig, which uses a high intensity laser for two-photon imaging, does not have eye pieces. A patch clamp experimentalist must construct a spatial map of how the $3 \mathrm{D}$ movement of the manipulator correlates with the movement of the $2 \mathrm{D}$ projection, which is a challenging feat. By integrating the motor positions of the digital micromanipulators, we developed a semi-automatic procedure to target specified regions of interest with a patch pipette. Along with the visual feedback provided by the World 3D interface, the semi-automatic procedures make it easy to place the pipette so as to target a 
neuron of interest, and thus enables patching deep in the slice. This is important, as neurons that are on the surface of the brain slice likely have many processes cut off during the slicing procedure and may not be a good representation of physiological neuronal activity. This system was developed at the beginning of my $\mathrm{PhD}$ and the software was subsequently used for all my experiments to create data maps of the recorded cells. The program has also helped speed up the training used to develop the fine motor skills required to properly position the pipette under visual guidance with the camera, however it does not teach experimenters the patient or the physics required to perform electrophysiology. Real-time analysis of the camera output for functional imaging allows for more up to date information on the progress of the experimenter.

In Chapter 4, I isolated $\mathrm{Na}^{+}$currents using whole cell voltage clamp in serotonergic ventral raphé neurons to examine if intracellular $\left[\mathrm{Ca}^{2+}\right]$ can modulate long-term inactivation of these neurons. By introducing $\mathrm{Ca}^{2+}$ chelators, EGTA and HEDTA, via the patch pipette, I recorded Nav channel steady-state activation, inactivation, recovery from inactivation and use dependent properties under these conditions. Minimal differences were found with spike generating, steady-state activation and inactivation properties as well as recovery from inactivation. Testing use dependence, via applying trains of depolarizing voltage pulses, indicates that high $\left[\mathrm{Ca}^{2+}\right]$ solutions significantly reduced the entry into inactivation. This suggests that, after repetitive use, Nav channels in high $\left[\mathrm{Ca}^{2+}\right]$ environments have reduced inactivation and increased availability. This conclusion was not easily predictable from existing data, as previous reports in heterologous systems demonstrate increased inactivation triggered by high $\left[\mathrm{Ca}^{2+}\right]$. In 
raphé neurons, long-term inactivation serves as a negative feedback loop that regulates the frequency of firing and thus it is predicted that $\mathrm{Ca}^{2+}$ could be a key signaling molecule that may be regulating this process.

Given my voltage clamp data, one would predict that, in current clamp, the overall activity would be increased with high $\left[\mathrm{Ca}^{2+}\right]$. However, finding intrinsically active raphé proved challenging with my high $\left[\mathrm{Ca}^{2+}\right]$ solution. This is not surprising, given that some experimenters suggest that patching under nominally zero $\mathrm{Ca}^{2+}$ is challenging (Marty and Neher, 1995). I remade the current clamp solutions many times to ensure that the result was not due to poor solution preparation, but the result persisted.

In neurons, Nav channel fast inactivation allows for the proper conduction of the action potential down the axon, creating a refractory period where the neuron cannot fire for a short period of time. This process prevents overexcitation and allows for neurons to maintain a steady firing frequency. In some cell types, Nav channels have unique inactivation properties, likely due to different expression profiles of Nav $\alpha$ subunits, which produce unique behaviors, including the persistent $\mathrm{Na}^{+}$current (French et al., 1990; Crill, 1996), the resurgent $\mathrm{Na}^{+}$current (Raman and Bean, 1997; Khaliq et al., 2003), and long-term inactivation (LTI) (Mickus et al., 1999; Goldfarb et al., 2007; Dover et al., 2010; Milescu et al., 2010b). These three mechanisms of inactivation are uniquely identifiable and serve to increase or decrease overall channel availability to produce faster or slower firing rates.

Found in many bursting neurons, the persistent $\mathrm{Na}^{+}$current $\left(\mathrm{I}_{\mathrm{NaP}}\right)$ describes an increased inward component of the Nav channel time course that does not inactivate, effectively 
always having available channels to conduct current. The presence of $\mathrm{I}_{\mathrm{NaP}}$ in neurons seems to be associated with the expression of the Nav1.6 subunit (Raman et al., 1997; Maurice et al., 2001; Chatelier et al., 2010; Tan and Soderlund, 2011), and is also regulated by $\beta$ subunits, as co-expression with $\beta 1$ and Nav 1.6 reduces current amplitude (Aman et al., 2009; Lopez-Santiago et al., 2011), whereas $\beta 3$ can lead to enhanced $\mathrm{I}_{\mathrm{NaP}}$ (Qu et al., 2001). $\mathrm{I}_{\mathrm{NaP}}$ is also regulated by $\mathrm{Ca}^{2+}$, as mutations in Nav 1.5 that prevent Calmodulin binding to the IQ domain on the C-terminus, enhances $\mathrm{I}_{\mathrm{NaP}}$ in HEK cells (Yan et al., 2017). Interestingly, in cases of spinal cord injury, where $\mathrm{I}_{\mathrm{NaP}}$ is upregulated in motoneurons, pharmacological blockade of calpain, a calcium-activated protease, prevents cleaving of Nav 1.6 and blunts the $\mathrm{I}_{\mathrm{NaP}}$ component, reducing muscle spasticity in treated mice (Brocard et al., 2016). These data suggest that the persistent component may be due to degradation or misfolding of Nav $\alpha$ subunit. However, in a recent publication on neurons in the pre-Bötzinger complex (Yamanishi et al., 2018), the $\mathrm{I}_{\mathrm{NaP}}$ component was isolated in both bursting and non-bursting neurons with cleverly designed voltage clamp protocols to identify the quasi steady-state activation and inactivation of the $\mathrm{I}_{\mathrm{NaP}}$ component, as well as the recovery from inactivation. These data confirm the idea that the kinetics do not change between bursters and nonbursters (Koizumi and Smith, 2008), and the behavior could be simply due to an increased amount of $\mathrm{I}_{\mathrm{NaP}}$, as a voltage clamp model can dynamically convert intrinsic bursters into nonbursters and nonbursters into bursters simply by introducing more or less $\mathrm{I}_{\mathrm{NaP}}$ (Yamanishi et al., 2018).

The resurgent $\mathrm{Na}^{+}$current is another behavior of Nav channels that provides extra depolarization to drive fast spiking $(40-50 \mathrm{~Hz})$ (Raman and Bean, 1997; Khaliq et al., 
2003). Unlike the persistent component, the resurgent current is elicited when repolarizing the membrane from $-20 \mathrm{mV}$ to $-60 \mathrm{mV}$, where there is normally little or no current. The hypothesis for this mechanism is an interaction with Nav $\beta 4$, where an intracellular motif interacts to block the pore, faster than the IFM motif (fast inactivation particle)(Grieco et al., 2005; Lewis and Raman, 2011), unbinding to allow for a second opening or a resurgence of current. Increasing the phosphorylation state of Nav channels regulates the kinetics and amplitude of resurgence (Raman and Bean, 2001; Grieco et al., 2002). The resurgent current is also attributed to the presence of Nav 1.6 and Nav $\beta 4$ (Raman et al., 1997), but the behavior can be induced by toxins that slow Nav channel inactivation (Grieco and Raman, 2004; Bant et al., 2013) and has reduced amplitude in SCN8A (Nav1.6) knockout mice (Do and Bean, 2004; Grieco and Raman, 2004; Levin et al., 2006), Nav $\beta 4$ knockout mice (Ransdell et al., 2017), and FGF14 KO mice (Yan et al., 2014). It seems that the mechanism of the resurgent behavior requires slower fast inactivation and an open channel blocker, but it is likely that more molecular activity is at play, as suggested by the inability to reproduce the behavior in heterologous systems.

The last modification of inactivation, long-term inactivation, potentially prevents neurons from firing too fast. In serotonergic raphé neurons, LTI can be triggered by a single fast depolarization, such as an action potential, but remains inactivated over much longer timescales (seconds vs milliseconds) (Milescu et al., 2010b). With each stimulus, a small proportion ( 20\%) of channels remain inactivated, increasing the length of the inter-spike interval and reducing firing frequency. Repetitive stimuli trigger LTI that requires longer time periods to fully recover. The mechanism of LTI can be reproduced in heterologous 
systems by co-expressing the A-isoform of fibroblast growth factor homologous factors (FHFs) with Nav1.6 channels (Dover et al., 2010). More evidence for this interaction lies in recordings of $\mathrm{Na}^{+}$currents from knockouts of FHFs that have reduced current density and faster recovery times (Goldfarb et al., 2007; Laezza et al., 2009).

Ideally, a kinetic mechanism should be able to explain any behavior the channel performs. Each behavioral modification described above are from recordings where channels have modified inactivation rates and often coincides with expression of Nav1.6. Interestingly, these behaviors can be described by the same model topology, the original Kuo and Bean topology with an added non-conducting state from the open state, however, with largely different rate constants (Raman et al., 1997; Magistretti et al., 2006; Dover et al., 2010; Milescu et al., 2010b; Yamanishi et al., 2018). However, these models do not maintain the same rates in the other states, as some models were handtweaked to describe the trends in the data, whereas others were fit to the data. With more complex model topologies, it is challenging to interpret how a single rate constant will affect the behavior of the model. However, in the case of the LTI model, hand tweaking the rates between the open state and extra state modifies the recovery from inactivation without drastically modifying the steady state properties. Interestingly, adding more states to the LTI state creating a longer chain does not seem to improve the fit. Overall, the existence of all these types of inactivation processes suggests that the Nav kinetic mechanism is complex and can be modulated for specific functions. Hence, it is important to take as much data as possible when searching for a good model. 
Considering all the data presented here, there could be many directions for future studies. To more clearly establish the effect of intracellular $\left[\mathrm{Ca}^{2+}\right]$ on Nav kinetics, one would have to record Nav currents under both low and high levels within the same cell, via $\mathrm{Ca}^{2+}$ uncaging. My preliminary attempts to examine the entry into inactivation before and after two-photon uncaging of DM-nitrophen have been inconclusive. Once the first uncaging event occurs, it is challenging to consistently maintain a high level of $\mathrm{Ca}^{2+}$ for more than a few milliseconds, as the buffering capacity for $\mathrm{Ca}^{2+}$ is quite fast. It is therefore challenging to test the long recovery protocol, which would be best at unveiling longterm inactivation effects. One could also use the perforated patch clamp technique, which does not rupture the membrane as in whole-cell recordings, but instead utilizes a drug to slowly create holes in the membrane, resulting in reasonably low access resistance. This technique does not dialyze the cell with the internal solution, thus washing away the intrinsic buffering proteins within the cell, providing recordings with "normal" intracellular $\left[\mathrm{Ca}^{2+}\right]$ levels.

Another direction would be to remove Nav channel fast inactivation via pronase or a conditional mutant that introduces a mutation in the IFM motif. Without the ability for Nav channels to inactivate, it would be interesting to see if further addition of FHF proteins or calmodulin or non-calcium binding (apo) calmodulin via the patch pipette could induce long-term inactivation. These experiments could potentially determine if long-term inactivation is in fact an open-state block or if it is rather a stabilization of the fast inactivation state. These studies ideally would be first done in heterologous expression system, which we have attempted to perform in Xenopus oocytes, however 
our current preparation with two-electrode voltage clamp is not fast enough to properly clamp the fast $\mathrm{Na}^{+}$currents. Ideally, one would use the cut-open oocyte technique, which allows for a faster clamp, as well as control over both internal and external solutions. These experiments could also help delineate other molecular interactions of long-term inactivation.

On the timescale of organism behavior (months or years), neurons are likely to fire countless action potentials, so much so that a single action potential gets lost in the noise. The mechanisms studied here are on much faster time scales, where the behaviors could be explained by the activity of the channel itself, rather than through changes of overall ion channel expression. Characterizing both long and short time scales is vital to fully understand the function of neurons. However, one must also try to limit the number of variables in the system. Due to limitations with keeping the preparation alive with slice electrophysiology, it is not possible to look at these long (>hour) timescales but this may become possible with in vivo recordings that use implantable devices. However, wholecell patch-clamp where proper voltage clamp can be applied is yet to be worked out in this system. Overall, one must design experiments that could lead to a clear answer and understanding of the results. To fully understand how neurons function on long-time scales, one must utilize other techniques, such as monitoring ion channel expression at the membrane, or protein trafficking within the neuron, to fully grasp the full function of neurons. Our work presented here is just a small step towards that understanding, and it will be interesting to see how other investigators will use these tools in the future. 


\section{References}

2015. QuB - The Mlab Version.

Adams, P.J., M. Ben-Johny, I.E. Dick, T. Inoue, and D.T. Yue. 2014. Apocalmodulin itself promotes ion channel opening and Ca 2+ regulation. Cell. 159:608-622.

Ahern, C.A., J. Payandeh, F. Bosmans, and B. Chanda. 2016. The hitchhiker's guide to the voltage-gated sodium channel galaxy. J. Gen. Physiol. 147:1-24.

Akerboom, J., T.-W. Chen, T.J. Wardill, L. Tian, J.S. Marvin, S. Mutlu, N.C. Calderón, F. Esposti, B.G. Borghuis, and X.R. Sun. 2012. Optimization of a GCaMP calcium indicator for neural activity imaging. J. Neurosci. 32:13819-13840.

Akk, G., L.S. Milescu, and M. Heckmann. 2005. Activation of heteroliganded mouse muscle nicotinic receptors. J. Physiol. 564:359-376.

Aman, T.K., and I.M. Raman. 2007. Subunit dependence of Na channel slow inactivation and open channel block in cerebellar neurons. Biophys. J. 92:1938-1951.

Aman, T.K., T.M. Grieco-Calub, C. Chen, R. Rusconi, E.A. Slat, L.L. Isom, and I.M. Raman. 2009. Regulation of persistent Na current by interactions between $\beta$ subunits of voltagegated Na channels. J. Neurosci. 29:2027-2042.

Aman, T.K., and I.M. Raman. 2010. Inwardly Permeating Na Ions Generate the Voltage Dependence of Resurgent Na Current in Cerebellar Purkinje Neurons. J. Neurosci. 30:5629-5634.

Anderson, C., and C. Stevens. 1973. Voltage clamp analysis of acetylcholine produced end-plate current fluctuations at frog neuromuscular junction. J. Physiol. 235:655-691.

Armstrong, C.M. 2006. Na channel inactivation from open and closed states. Proc. Natl. Acad. Sci. USA. 103:17991-17996.

Armstrong, C.M., and F. Bezanilla. 1974. Charge movement associated with the opening and closing of the activation gates of the Na channels. J. Gen. Physiol. 63:533-552.

Armstrong, C.M., and F. Bezanilla. 1977. Inactivation of the sodium channel. II. Gating current experiments. J. Gen. Physiol. 70:567-590.

Armstrong, C.M., F. Bezanilla, and E. Rojas. 1973. Destruction of sodium conductance inactivation in squid axons perfused with pronase. J. Gen. Physiol. 62:375-391.

Armstrong, C.M., and B. Hille. 1998. Voltage-gated ion channels and electrical excitability. Neuron. 20:371-380. 
Armstrong, C.M., and W.F. Gilly. 1979. Fast and slow steps in the activation of sodium channels. J. Gen. Physiol. 74:691-711.

Ashkin, A. 1970. Acceleration and trapping of particles by radiation pressure. Phys. Rev. Lett. $24: 156$

Ashkin, A., J.M. Dziedzic, J. Bjorkholm, and S. Chu. 1986. Observation of a single-beam gradient force optical trap for dielectric particles. Optics letters. 11:288-290.

Athanasiu, L., M. Mattingsdal, A.K. Kähler, A. Brown, O. Gustafsson, I. Agartz, I. Giegling, P. Muglia, S. Cichon, and M. Rietschel. 2010. Gene variants associated with schizophrenia in a Norwegian genome-wide study are replicated in a large European cohort. J.Psych. Res. 44:748-753.

Bading, H., D.D. Ginty, and M.E. Greenberg. 1993. Regulation of gene expression in hippocampal neurons by distinct calcium signaling pathways. Science. 260:181-186.

Ball, F.G., and M.S. Sansom. 1989. Ion-channel gating mechanisms: model identification and parameter estimation from single channel recordings. Proc. R. Soc. Lond. B Biol. Sci. 236:385-416.

Bandyopadhyay, S., and S. Saha. 2013. Some single-and multiobjective optimization techniques. In Unsupervised Classification. Springer. 17-58.

Bant, J.S., and I.M. Raman. 2010. Control of transient, resurgent, and persistent current by openchannel block by Na channel beta 4 in cultured cerebellar granule neurons. Proc. Natl. Acad. Sci. USA. 107:12357-12362

Bant, J.S., T.K. Aman, and I.M. Raman. 2013. Antagonism of lidocaine inhibition by openchannel blockers that generate resurgent Na current. J. Neurosci. 33:4976-4987.

Baranauskas, G., Y. David, and I.A. Fleidervish. 2013. Spatial mismatch between the Na+ flux and spike initiation in axon initial segment. Proc. Natl. Acad. Sci. USA. 110:4051-4056.

Baranauskas, G., and M. Martina. 2006. Sodium currents activate without a Hodgkin-andHuxley-type delay in central mammalian neurons. J. Neurosci. 26:671-684.

Bar-Yehuda, D., and A. Korngreen. 2008. Space-clamp problems when voltage clamping neurons expressing voltage-gated conductances. J. Neurophysiol. 99:1127-1136.

Baum, L.E., T. Petrie, G. Soules, and N. Weiss. 1970. A maximization technique occurring in the statistical analysis of probabilistic functions of Markov chains. Ann. Math. Stat. 41:164171.

Bean, B.P. 2007. The action potential in mammalian central neurons. Nat. Rev. Neurosci. 8:451465. 
Bélanger, M., I. Allaman, and P.J. Magistretti. 2011. Brain energy metabolism: focus on astrocyte-neuron metabolic cooperation. Cell metabolism. 14:724-738.

Ben-Johny, M., I. E Dick, L. Sang, W. B Limpitikul, P. Wei Kang, J. Niu, R. Banerjee, W. Yang, J. S Babich, and J. B Issa. 2015. Towards a unified theory of calmodulin regulation (calmodulation) of voltage-gated calcium and sodium channels. Curr. Mol. Pharm. 8:188-205.

Ben-Johny, M., P.S. Yang, H. Bazzazi, and D.T. Yue. 2013. Dynamic switching of calmodulin interactions underlies $\mathrm{Ca} 2+$ regulation of $\mathrm{CaV} 1.3$ channels. Nat. Commun. 4:1717.

Ben-Johny, M., P.S. Yang, J. Niu, W. Yang, R. Joshi-Mukherjee, and D.T. Yue. 2014. Conservation of $\mathrm{Ca} 2+/$ Calmodulin Regulation across $\mathrm{Na}$ and $\mathrm{Ca} 2+$ Channels. Cell. 157:1657-1670.

Bender, K.J., V.N. Uebele, J.J. Renger, and L.O. Trussell. 2012. Control of firing patterns through modulation of axon initial segment T-type calcium channels. J. Physiol. 590:109118.

Benndorf, K. 1988. Patch clamp analysis of recovery of sodium channels from inactivation in mammalian myocardium in terms of a Markovian state model. Biomedica biochimica acta. 48:287-302.

Bertsekas, D.P. 1975. Nondifferentiable optimization via approximation. In Nondifferentiable optimization. Springer. 1-25.

Bezanilla, F. 2000. The voltage sensor in voltage-dependent ion channels. Physiol. Revs. 80:555592.

Bezanilla, F., and C.M. Armstrong. 1977. Inactivation of the sodium channel. I. Sodium current experiments. J. Gen. Physiol. 70:549-566.

Biswas, S., D. DiSilvestre, Y. Tian, V.L. Halperin, and G.F. Tomaselli. 2009. Calcium-mediated dual-mode regulation of cardiac sodium channel gating. Circ. Res. 104:870-878.

Box, G.E. 1979. Robustness in the strategy of scientific model building. Robustness in statistics. $1: 201-236$.

Brackenbury, W.J., and L.L. Isom. 2008. Voltage-gated Na+ channels: potential for beta subunits as therapeutic targets. Expert. Opin. Ther. Targets. 12:1191-1203.

Brocard, C., V. Plantier, P. Boulenguez, S. Liabeuf, M. Bouhadfane, A. Viallat-Lieutaud, L. Vinay, and F. Brocard. 2016. Cleavage of $\mathrm{Na}+$ channels by calpain increases persistent $\mathrm{Na}+$ current and promotes spasticity after spinal cord injury. Nat. Med. 22:404-411. 
Bruno, W.J., J. Yang, and J.E. Pearson. 2005. Using independent open-to-closed transitions to simplify aggregated Markov models of ion channel gating kinetics. Proc. Natl. Acad. Sci. USA. 102:6326-6331.

Buffington, S.A., and M.N. Rasband. 2011. The axon initial segment in nervous system disease and injury. Eur. J. Neurosci. 34:1609-1619.

Burzomato, V., M. Beato, P.J. Groot-Kormelink, D. Colquhoun, and L.G. Sivilotti. 2004. Singlechannel behavior of heteromeric $\alpha 1 \beta$ glycine receptors: an attempt to detect a conformational change before the channel opens. J. Neurosci. 24:10924-10940.

Bustamante, C., J.C. Macosko, and G.J. Wuite. 2000. Grabbing the cat by the tail: manipulating molecules one by one. Nat. Rev. Mol. Cell Biol. 1:130.

Butera Jr, R.J., C.G. Wilson, C. DelNegro, and J.C. Smith. 2001. A methodology for achieving high-speed rates for artificial conductance injection in electrically excitable biological cells. Biomed. Eng. IEEE Trans. 48:1460-1470.

Calderhead, B., M. Epstein, L. Sivilotti, and M. Girolami. 2013. Bayesian approaches for mechanistic ion channel modeling. In Silico Systems Biology.247-272.

Cantrell, A.R., V.C. Tibbs, H.Y. Frank, B.J. Murphy, E.M. Sharp, Y. Qu, W.A. Catterall, and T. Scheuer. 2002. Molecular mechanism of convergent regulation of brain $\mathrm{Na}+$ channels by protein kinase $\mathrm{C}$ and protein kinase A anchored to AKAP-15. Mol. Cell. Neurosci. 21:6380.

Capes, D.L., M.P. Goldschen-Ohm, M. Arcisio-Miranda, F. Bezanilla, and B. Chanda. 2013. Domain IV voltage-sensor movement is both sufficient and rate limiting for fast inactivation in sodium channels. J. Gen. Physiol. 142:101-112.

Carter, B.C., and B.P. Bean. 2009. Sodium entry during action potentials of mammalian neurons: incomplete inactivation and reduced metabolic efficiency in fast-spiking neurons. Neuron. 64:898-909.

Catterall, W.A., A.L. Goldin, and S.G. Waxman. 2005. International Union of Pharmacology. XLVII. Nomenclature and structure-function relationships of voltage-gated sodium channels. Pharmacol. Rev. 57:397-409.

Catterall, W.A., S. Cestèle, V. Yarov-Yarovoy, H.Y. Frank, K. Konoki, and T. Scheuer. 2007. Voltage-gated ion channels and gating modifier toxins. Toxicon. 49:124-141.

Catterall, W.A., I.M. Raman, H.P. Robinson, T.J. Sejnowski, and O. Paulsen. 2012. The Hodgkin-Huxley heritage: from channels to circuits. J. Neurosci. 32:14064-14073. 
Celentano, J.J., and A.G. Hawkes. 2004. Use of the covariance matrix in directly fitting kinetic parameters: application to GABAA receptors. Biophys. J. 87:276-294.

Chahine, M., and M.E. O'Leary. 2011. Regulatory Role of Voltage-Gated Na Channel beta Subunits in Sensory Neurons. Front. Pharmacol. 2:70.

Chahine, M., R. Ziane, K. Vijayaragavan, and Y. Okamura. 2005. Regulation of Nav channels in sensory neurons. Trends Pharm Sci. 26:496-502.

Chanda, B., and F. Bezanilla. 2002a. Changes in voltage dependent fluorescence in domain IV follow other domains in the skeletal muscle sodium channel. Biophys. J. 82:174a-174a.

Chanda, B., and F. Bezanilla. 2002b. Tracking voltage-dependent conformational changes in skeletal muscle sodium channel during activation. J. Gen. Physiol. 120:629-645.

Chang, A., F. Abderemane-Ali, G.L. Hura, N.D. Rossen, R.E. Gate, and D.L. Minor Jr. 2018. A Calmodulin C-Lobe Ca2+-Dependent Switch Governs Kv7 Channel Function. Neuron. 97:836-852. e836.

Chang, K.-J., and M.N. Rasband. 2013. Excitable domains of myelinated nerves: axon initial segments and nodes of Ranvier. Curr. Top. Membr. 72:159-192.

Chatelier, A., J. Zhao, P. Bois, and M. Chahine. 2010. Biophysical characterisation of the persistent sodium current of the Nav1.6 neuronal sodium channel: a single-channel analysis. Pflugers Archiv : Eur. J. Physiol. 460:77-86.

Chen, T.-c., B. Law, T. Kondratyuk, and S. Rossie. 1995. Identification of soluble protein phosphatases that dephosphorylate voltage-sensitive sodium channels in rat brain. J. Biol. Chem. 270:7750-7756.

Chen, T.-W., T.J. Wardill, Y. Sun, S.R. Pulver, S.L. Renninger, A. Baohan, E.R. Schreiter, R.A. Kerr, M.B. Orger, and V. Jayaraman. 2013. Ultrasensitive fluorescent proteins for imaging neuronal activity. Nature. 499:295.

Chin, D., and A.R. Means. 2000. Calmodulin: a prototypical calcium sensor. Trends Cell Biol. 10:322-328.

Christini, D.J., K.M. Stein, S.M. Markowitz, and B.B. Lerman. 1999. Practical real-time computing system for biomedical experiment interface. Ann. Biomed. Eng. 27:180-186.

Colbert, C.M., and D. Johnston. 1996. Axonal action-potential initiation and $\mathrm{Na}+$ channel densities in the soma and axon initial segment of subicular pyramidal neurons. $J$. Neurosci. 16:6676-6686. 
Colbert, C.M., J.C. Magee, D.A. Hoffman, and D. Johnston. 1997. Slow recovery from inactivation of $\mathrm{Na}+$ channels underlies the activity-dependent attenuation of dendritic action potentials in hippocampal CA1 pyramidal neurons. J. Neurosci. 17:6512-6521.

Colquhoun, D., A.G. Hawkes, and K. Srodzinski. 1996. Joint distributions of apparent open times and shut times of single ion channels and the maximum likelihood fitting of mechanisms. Phil. Trans. R. Soc. Lond. A. 354:2555-2590.

Colquhoun, D., and A.G. Hawkes. 1982. On the stochastic properties of bursts of single ion channel openings and of clusters of bursts. Phil. Trans. R. Soc. Lond. B. Biol. Sci. 300:1-59.

Colquhoun, D., and A.G. Hawkes. 1995a. The Principles of the Stochastic Interpretation of Ionchannel mechanisms. In Single-channel recording. B. Sakmann and E. Neher, editors. Plenum Press, New York. 397-479.

Colquhoun, D., and A.G. Hawkes. 1995b. A Q-matrix cookbook: how to write only one program to calculate the single-channel and macroscopic predictions for any kinetic mechanism. In Single-channel recording. B. Sakmann and E. Neher, editors. Plenum Press, New York. 589-636.

Colquhoun, D., K.A. Dowsland, M. Beato, and A.J.R. Plested. 2004. How to impose microscopic reversibility in complex reaction mechanisms. Biophys. J. 86:3510-3518.

Colquhoun, D., and F. Sigworth. 1995. Fitting and statistical analysis of single-channel records. In Single-channel recording. B. Sakmann and E. Neher, editors. Plenum Press, New York. 483-587.

Connors, B.W., and M.J. Gutnick. 1990. Intrinsic firing patterns of diverse neocortical neurons. Trends Neurosci. 13:99-104.

Cook, E.P., and J.H. Maunsell. 2002. Dynamics of neuronal responses in macaque MT and VIP during motion detection. Nat. Neurosci. 5:985.

Corcoran, A.E., M.R. Hodges, Y. Wu, W. Wang, C.J. Wylie, E.S. Deneris, and G.B. Richerson. 2009. Medullary serotonin neurons and central CO 2 chemoreception. Respir. Physiol. Neurobiol. 168:49-58.

Costa, M., and W.A. Catterall. 1984. Cyclic AMP-dependent phosphorylation of the alpha subunit of the sodium channel in synaptic nerve ending particles. J. Biol. Chem. 259:8210-8218.

Cox, D., and H. Miller. 1965. The theory of stochastic processes, Methuen \& Co. Ltd, London, $U K$. 
Crill, W.E. 1996. Persistent sodium current in mammalian central neurons. Annu Rev Physiol. 58:349-362.

Csanady, L. 2006. Statistical evaluation of ion-channel gating models based on distributions of log-likelihood ratios. Biophys. J. 90:3523-3545.

de Oliveira, R.B., M.C. Howlett, F.S. Gravina, M.S. Imtiaz, R.J. Callister, A.M. Brichta, and D.F. van Helden. 2010. Pacemaker currents in mouse locus coeruleus neurons. Neurosci. 170:166-177.

Deschênes, I., N. Neyroud, D. DiSilvestre, E. Marbán, D.T. Yue, and G.F. Tomaselli. 2002. Isoform-specific modulation of voltage-gated $\mathrm{Na}+$ channels by calmodulin. Circ. Res. 90:e49-e57.

Deschênes, I., E. Trottier, and M. Chahine. 1999. Cysteine scanning analysis of the IFM cluster in the inactivation gate of a human heart sodium channel. Cardiovasc. res. 42:521-529.

Do, M.T.H., and B.P. Bean. 2003. Subthreshold sodium currents and pacemaking of subthalamic neurons: modulation by slow inactivation. Neuron. 39:109-120.

Do, M.T.H., and B.P. Bean. 2004. Sodium currents in subthalamic nucleus neurons from Nav1. 6null mice. J Neurophysiol. 92:726-733.

Dolmetsch, R.E., K. Xu, and R.S. Lewis. 1998. Calcium oscillations increase the efficiency and specificity of gene expression. Nature. 392:933.

Dorval, A.D., D.J. Christini, and J.A. White. 2001. Real-time linux dynamic clamp: a fast and flexible way to construct virtual ion channels in living cells. Ann. Biomed. Eng. 29:897907.

Dover, K., S. Solinas, E. D’Angelo, and M. Goldfarb. 2010. Long-term inactivation particle for voltage-gated sodium channels. J. Physiol. 588:3695-3711.

Druckmann, S., Y. Banitt, A. Gidon, F. Schürmann, H. Markram, and I. Segev. 2007. A novel multiple objective optimization framework for constraining conductance-based neuron models by experimental data. Front. Neurosci. 1:7.

Eaholtz, G., T. Scheuer, and W.A. Catterall. 1994. Restoration of inactivation and block of open sodium channels by an inactivation gate peptide. Neuron. 12:1041-1048.

Edwards, F.A., A. Konnerth, B. Sakmann, and T. Takahashi. 1989. A thin slice preparation for patch clamp recordings from neurones of the mammalian central nervous system. Pflügers Archiv. 414:600-612.

Elliott, A., and J. Elliott. 1993. Characterization of TTX-sensitive and TTX-resistant sodium currents in small cells from adult rat dorsal root ganglia. J. Physiol. 463:39-56. 
Elston, T., H. Wang, and G. Oster. 1998. Energy transduction in ATP synthase. Nature. 391:510.

Epstein, M., B. Calderhead, M.A. Girolami, and L.G. Sivilotti. 2016. Bayesian statistical inference in ion-channel models with exact missed event correction. Biophys. J. 111:333348.

Evans, M.D., A.S. Dumitrescu, D.L. Kruijssen, S.E. Taylor, and M.S. Grubb. 2015. Rapid modulation of axon initial segment length influences repetitive spike firing. Cell Rep. $13: 1233-1245$.

Evans, M.D., R.P. Sammons, S. Lebron, A.S. Dumitrescu, T.B. Watkins, V.N. Uebele, J.J. Renger, and M.S. Grubb. 2013. Calcineurin signaling mediates activity-dependent relocation of the axon initial segment. J. Neurosci. 33:6950-6963.

Eyring, H. 1935. The activated complex in chemical reactions. J. Chem. Phys. 3:107-115.

Faas, G.C., S. Raghavachari, J.E. Lisman, and I. Mody. 2011. Calmodulin as a direct detector of Ca 2+ signals. Nat. Neurosci. 14:301.

Fleidervish, I.A., A. Friedman, and M.J. Gutnick. 1996. Slow inactivation of Na+ current and slow cumulative spike adaptation in mouse and guinea-pig neocortical neurones in slices. J. Physiol. 493:83-97.

Fletcher, R. 2013. Practical methods of optimization. John Wiley \& Sons.

Fletcher, R., and M.J.D. Powell. 1963. A rapidly convergent descent method for minimization. Computing J. 2:163-168.

Frank, H.Y., and W.A. Catterall. 2004. The VGL-chanome: a protein superfamily specialized for electrical signaling and ionic homeostasis. Science Signal. 2004:re15.

Frank, H.Y., V. Yarov-Yarovoy, G.A. Gutman, and W.A. Catterall. 2005. Overview of molecular relationships in the voltage-gated ion channel superfamily. Pharm. Revs. 57:387-395.

French, C.R., P. Sah, K.J. Buckett, and P.W. Gage. 1990. A voltage-dependent persistent sodium current in mammalian hippocampal neurons. J. Gen. Physiol. 95:1139-1157.

Gagniuc, P.A. 2017. Markov chains: from theory to implementation and experimentation. John Wiley \& Sons.

Gardos, G. 1958. The function of calcium in the potassium permeability of human erythrocytes. Biochim. Biophys. Acta. 30:653.

Gasser, A., T.S.-Y. Ho, X. Cheng, K.-J. Chang, S.G. Waxman, M.N. Rasband, and S.D. Dib-Hajj. 2012. An ankyrinG-binding motif is necessary and sufficient for targeting Nav1. 6 sodium channels to axon initial segments and nodes of Ranvier. J. Neurosci. 32:72327243. 
Getting, P.A. 1989. Emerging principles governing the operation of neural networks. Ann. Rev. Neurosci. 12:185-204.

Gnanasambandam, R., P. Gottlieb, and F. Sachs. 2017. The Kinetics and the Permeation Properties of Piezo Channels. In Current Topics in Membranes. P. Gottlieb, editor. Elsevier, Oxford. 275-307.

Goetz, R., K. Dover, F. Laezza, N. Shtraizent, X. Huang, D. Tchetchik, A.V. Eliseenkova, C.-F. Xu, T.A. Neubert, and D.M. Ornitz. 2009. Crystal structure of a fibroblast growth factor homologous factor (FHF) defines a conserved surface on FHFs for binding and modulation of voltage-gated sodium channels. J. Biol. Chem. 284:17883-17896.

Goldschen-Ohm, M.P., and B. Chanda. 2015. How to open a proton pore-more than S4? Nat. Struct. Mol. Biol. 22:277-278.

Golub, G.H., and C. Reinsch. 1970. Singular value decomposition and least squares solutions. Numerische mathematik. 14:403-420.

González, C., D. Baez-Nieto, I. Valencia, I. Oyarzún, P. Rojas, D. Naranjo, and R. Latorre. 2012. K (+) channels: function-structural overview. Compr Physiol. 2:2087-2149.

Goldfarb, M. 2005. Fibroblast growth factor homologous factors: evolution, structure, and function. Cytokine \& growth factor reviews. 16:215-220.

Goldfarb, M. 2012. Voltage-gated sodium channel-associated proteins and alternative mechanisms of inactivation and block. Cell. Mol. Life. Sci. 69:1067-1076.

Goldfarb, M., J. Schoorlemmer, A. Williams, S. Diwakar, Q. Wang, X. Huang, J. Giza, D. Tchetchik, K. Kelley, A. Vega, G. Matthews, P. Rossi, D.M. Ornitz, and E. D'Angelo. 2007. Fibroblast growth factor homologous factors control neuronal excitability through modulation of voltage-gated sodium channels. Neuron. 55:449-463.

Gorman, A., and M. Thomas. 1978. Changes in the intracellular concentration of free calcium ions in a pace - maker neurone, measured with the metallochromic indicator dye arsenazo III. J. Physiol. 275:357-376.

Grace, A.A., and B.S. Bunney. 1983. Intracellular and extracellular electrophysiology of nigral dopaminergic neurons--1. Identification and characterization. Neurosci. 10:301-315.

Grieco, T.M., F.S. Afshari, and I.M. Raman. 2002. A role for phosphorylation in the maintenance of resurgent sodium current in cerebellar Purkinje neurons. J. Neurosci. 22:3100-3107.

Grieco, T.M., and I.M. Raman. 2004. Production of resurgent current in Na(V)1.6-null Purkinje neurons by slowing sodium channel inactivation with beta-pompilidotoxin. J. Neurosci. 24:35-42. 
Grieco, T.M., J.D. Malhotra, C. Chen, L.L. Isom, and I.M. Raman. 2005. Open-channel block by the cytoplasmic tail of sodium channel $\beta 4$ as a mechanism for resurgent sodium current. Neuron. 45:233-244.

Grillner, S. 2003. The motor infrastructure: from ion channels to neuronal networks. Nat. Rev. Neurosci. 4:573-586.

Groschner, L.N., L. Chan Wah Hak, R. Bogacz, S. DasGupta, and G. Miesenbock. 2018. Dendritic Integration of Sensory Evidence in Perceptual Decision-Making. Cell. 173:894-905 e813.

Grosman, C., F.N. Salamone, S.M. Sine, and A. Auerbach. 2000. The extracellular linker of muscle acetylcholine receptor channels is a gating control element. J. Gen. Physiol. 116:327-340.

Grubb, M.S., and J. Burrone. 2010. Building and maintaining the axon initial segment. Curr. Op. Neurobiol. 20:481-488.

Grubb, M.S., Y. Shu, H. Kuba, M.N. Rasband, V.C. Wimmer, and K.J. Bender. 2011. Short- and long-term plasticity at the axon initial segment. J. Neurosci. 31:16049-16055.

Gurkiewicz, M., and A. Korngreen. 2007. A numerical approach to ion channel modelling using whole-cell voltage-clamp recordings and a genetic algorithm. PLoS Comput. Biol. 3:1633-1647.

Hamill, O.P., A. Marty, E. Neher, B. Sakmann, and F.J. Sigworth. 1981. Improved patch-clamp techniques for high-resolution current recording from cells and cell-free membrane patches. Pflugers Arch. 391:85-100.

Harris-Warrick, R.M., and E. Marder. 1991. Modulation of neural networks for behavior. Ann. Rev. Neurosci. 14:39-57.

Hartzell, C., I. Putzier, and J. Arreola. 2005. Calcium-activated chloride channels. Annu. Rev. Physiol. 67:719-758.

Hasan, R., A.T. Leeson-Payne, J.H. Jaggar, and X. Zhang. 2017. Calmodulin is responsible for Ca 2+-dependent regulation of TRPA1 Channels. Sci. Rep. 7:45098.

Hasenstaub, A., S. Otte, E. Callaway, and T.J. Sejnowski. 2010. Metabolic cost as a unifying principle governing neuronal biophysics. Proc. Natl. Acad. Sci. USA. 107:12329-12334.

Hawkes, A.G., A. Jalali, and D. Colquhoun. 1990. The Distributions of the Apparent Open Times and Shut Times in a Single Channel Record When Brief Events Cannot Be Detected. Philos. Roy. Soc. A. 332:511-538. 
Hawkes, A.G., A. Jalali, and D. Colquhoun. 1992. Asymptotic Distributions of Apparent Open Times and Shut Times in a Single Channel Record Allowing for the Omission of Brief Events. Philos. Roy. Soc. B. 337:383-404.

Hazama, A., and Y. Okada. 1988. Ca2+ sensitivity of volume-regulatory $\mathrm{K}+$ and $\mathrm{Cl}$-channels in cultured human epithelial cells. J. Physiol. 402:687-702.

Heinemann, S.H. 1995. Guide to data acquisition and analysis. In Single-Channel recording. B. Sakmann and E. Neher, editors. Springer. 53-91.

Heinemann, S.H., and F. Conti. 1992. Nonstationary noise analysis and application to patch clamp recordings. Meth. Enzymol. 207:131-148.

Helmchen, F., K. Imoto, and B. Sakmann. 1996. Ca2+ buffering and action potential-evoked Ca2+ signaling in dendrites of pyramidal neurons. Biophys. J. 70:1069-1081.

Helmchen, F., and W. Denk. 2005. Deep tissue two-photon microscopy. Nature Methods. 2:932940.

Herring, B.E., and R.A. Nicoll. 2016. Long-term potentiation: from CaMKII to AMPA receptor trafficking. Ann. Rev. Physiol. 78:351-365.

Herzog, R.I., C. Liu, S.G. Waxman, and T.R. Cummins. 2003. Calmodulin binds to the C terminus of sodium channels Nav1. 4 and Nav1. 6 and differentially modulates their functional properties. J. Neurosci. 23:8261-8270.

Hille, B. 2001. Ion channels of excitable membranes. Sinauer Sunderland, MA.

Hidalgo, P., and R. MacKinnon. 1995. Revealing the architecture of a K+ channel pore through mutant cycles with a peptide inhibitor. Science. 268:307-310.

Himmelblau, D.M. 1972. Applied nonlinear programming. McGraw-Hill Companies.

Hines, K.E., T.R. Middendorf, and R.W. Aldrich. 2014. Determination of parameter identifiability in nonlinear biophysical models: A Bayesian approach. J. Gen. Physiol. 201311116.

Hinman, J.D., M.N. Rasband, and S.T. Carmichael. 2013. Remodeling of the axon initial segment after focal cortical and white matter stroke. Stroke. 44:182-189.

Hirn, C., G. Shapovalov, O. Petermann, E. Roulet, and U.T. Ruegg. 2008. Nav1. 4 deregulation in dystrophic skeletal muscle leads to Na+ overload and enhanced cell death. J. Gen. Physiol. 132:199-208.

Hodges, M.R., M. Wehner, J. Aungst, J.C. Smith, and G.B. Richerson. 2009. Transgenic mice lacking serotonin neurons have severe apnea and high mortality during development. $J$. Neurosci. 29:10341-10349. 
Hodgkin, A.L., and A.F. Huxley. 1952. A quantitative description of membrane current and its application to conduction and excitation in nerve. J. Physiol. Lond. 117:500-544.

Horn, R., S. Ding, and H.J. Gruber. 2000. Immobilizing the moving parts of voltage-gated ion channels. J. Gen. Physiol.. 116:461-476.

Horn, R., and K. Lange. 1983. Estimating kinetic constants from single channel data. Biophys. J. 43:207-223.

Horn, R., and C.A. Vandenberg. 1984. Statistical properties of single sodium channels. J. Gen. Physiol. 84:505-534.

Hoshi, T., W.N. Zagotta, and R.W. Aldrich. 1994. Shaker potassium channel gating. I: Transitions near the open state. J. Gen. Physiol. 103:249-278.

Hu, W., C. Tian, T. Li, M. Yang, H. Hou, and Y. Shu. 2009. Distinct contributions of Nav1. 6 and Nav1. 2 in action potential initiation and backpropagation. Nat. Neurosci. 12:9961002.

Hülsmann, S., Y. Oku, W. Zhang, and D.W. Richter. 2000. Metabolic coupling between glia and neurons is necessary for maintaining respiratory activity in transverse medullary slices of neonatal mouse. Eur. J. Neurosci. 12:856-862.

Insel, T.R., S.C. Landis, and F.S. Collins. 2013. The NIH brain initiative. Science. 340:687-688.

Izhikevich, E.M., and G.M. Edelman. 2008. Large-scale model of mammalian thalamocortical systems. Proc. Natl. Acad. Sci. USA. 105:3593-3598.

Jacobs, B.L., and E.C. Azmitia. 1992. Structure and function of the brain serotonin system. Physiol. Rev. 72:165-229.

Joiner, W.J., R. Khanna, L.C. Schlichter, and L.K. Kaczmarek. 2001. Calmodulin regulates assembly and trafficking of SK4/IK1 Ca2+-activated K+ channels. J Biol. Chem. 276:37980-37985.

Jung, H.-Y., T. Mickus, and N. Spruston. 1997. Prolonged sodium channel inactivation contributes to dendritic action potential attenuation in hippocampal pyramidal neurons. $J$. Neurosci. 17:6639-6646.

Jung, J., J.H. Nam, H.W. Park, U. Oh, J.-H. Yoon, and M.G. Lee. 2013. Dynamic modulation of ANO1/TMEM16A HCO3- permeability by Ca2+/calmodulin. Proc. Natl. Acad. Sci. USA. 110:360-365.

Kaczmarek, L.K. 2006. Non-conducting functions of voltage-gated ion channels. Nat. Rev. Neurosci. 7:761-771. 
Kaiser, C.M., D.H. Goldman, J.D. Chodera, I. Tinoco, and C. Bustamante. 2011. The ribosome modulates nascent protein folding. Science. 334:1723-1727.

Kao, J., A.T. Harootunian, and R.Y. Tsien. 1989. Photochemically generated cytosolic calcium pulses and their detection by fluo-3. J. Biol. Chem. 264:8179-8184.

Kandel, E.R. 2012. The molecular biology of memory: cAMP, PKA, CRE, CREB-1, CREB-2, and CPEB. Mol.Brain. 5:14.

Kaphzan, H., S.A. Buffington, J.I. Jung, M.N. Rasband, and E. Klann. 2011. Alterations in intrinsic membrane properties and the axon initial segment in a mouse model of Angelman syndrome. J. Neurosci. 31:17637-17648.

Kaphzan, H., S.A. Buffington, A.B. Ramaraj, J.B. Lingrel, M.N. Rasband, E. Santini, and E. Klann. 2013. Genetic reduction of the $\alpha 1$ subunit of $\mathrm{Na} / \mathrm{K}$-ATPase corrects multiple hippocampal phenotypes in Angelman syndrome. Cell Rep. 4:405-412.

Katz, B., and R. Miledi. 1968. The role of calcium in neuromuscular facilitation. J Physiol. 195:481-492.

Keen, J.E., R. Khawaled, D.L. Farrens, T. Neelands, A. Rivard, C.T. Bond, A. Janowsky, B. Fakler, J.P. Adelman, and J. Maylie. 1999. Domains responsible for constitutive and $\mathrm{Ca} 2+-$ dependent interactions between calmodulin and small conductance $\mathrm{Ca} 2+$-activated potassium channels. J. Neurosci. 19:8830-8838.

Kienker, P. 1989. Equivalence of aggregated Markov models of ion-channel gating. Proc. R. Soc. Lond. B Biol. Sci. 236:269-309.

Khaliq, Z.M., N.W. Gouwens, and I.M. Raman. 2003. The contribution of resurgent sodium current to high-frequency firing in Purkinje neurons: an experimental and modeling study. J. Neurosci.. 23:4899-4912.

Knight, B.W. 1972. Dynamics of encoding in a population of neurons. J. Gen. Physiol. 59:734-766.

Koch, C., and I. Segev. 2000. The role of single neurons in information processing. Nat. Neurosci. 3:1171-1177.

Koizumi, H., N. Koshiya, J.X. Chia, F. Cao, J. Nugent, R. Zhang, and J.C. Smith. 2013. Structural-functional properties of identified excitatory and inhibitory interneurons within pre-Bötzinger complex respiratory microcircuits. J. Neurosci. 33:2994-3009.

Koizumi, H., and J.C. Smith. 2008. Persistent Na+ and K+-dominated leak currents cntribute to respiratory rhythm generation in the pre-Bötzinger complex in vitro. J. Neurosci. 28:1773-1785. 
Kolb, I., C. Landry, M. Yip, C. Lewallen, W. Stoy, J. Lee, A. Felouzis, B. Yang, E. Boyden, and C.J. Rozell. 2019. PatcherBot: a single-cell electrophysiology robot for adherent cells and brain slices. J. Neural Eng.

Kole, M.H., S.U. Ilschner, B.M. Kampa, S.R. Williams, P.C. Ruben, and G.J. Stuart. 2008. Action potential generation requires a high sodium channel density in the axon initial segment. Nat. Neurosci. 11:178-186.

Kole, M.H., and G.J. Stuart. 2012. Signal processing in the axon initial segment. Neuron. 73:235-247.

Koos, K., J. Molnár, and P. Horvath. 2017. Pipette Hunter: Patch-Clamp Pipette Detection. In Scandinavian Conference on Image Analysis. Springer. 172-183.

Koshiya, N., and J.C. Smith. 1999. Neuronal pacemaker for breathing visualized in vitro. Nature. 400:360-363.

Kuba, H. 2012. Structural tuning and plasticity of the axon initial segment in auditory neurons. $J$. Physiol. 590:5571-5579.

Kuba, H., R. Adachi, and H. Ohmori. 2014. Activity-dependent and activity-independent development of the axon initial segment. J. Neurosci. 34:3443-3453.

Kullmann, P.H., D.W. Wheeler, J. Beacom, and J.P. Horn. 2004. Implementation of a fast 16-bit dynamic clamp using LabVIEW-RT. J. Neurophysiol. 91:542-554.

Kuo, C.C., and B.P. Bean. 1994. Na+ channels must deactivate to recover from inactivation. Neuron. 12:819-829.

Laezza, F., B.R. Gerber, J.Y. Lou, M.A. Kozel, H. Hartman, A.M. Craig, D.M. Ornitz, and J.M. Nerbonne. 2007. The FGF14(F145S) mutation disrupts the interaction of FGF14 with voltage-gated $\mathrm{Na}+$ channels and impairs neuronal excitability. J. Neurosci. 27:1203312044.

Laezza, F., A. Lampert, M.A. Kozel, B.R. Gerber, A.M. Rush, J.M. Nerbonne, S.G. Waxman, S.D. Dib-Hajj, and D.M. Ornitz. 2009. FGF14 N-terminal splice variants differentially modulate Nav1.2 and Nav1.6-encoded sodium channels. Mol. Cell. Neurosci. 42:90-101.

Lai, H.C., and L.Y. Jan. 2006. The distribution and targeting of neuronal voltage-gated ion channels. Nat. Rev. Neurosci. 7:548-562.

Lambers, T.T., A.F. Weidema, B. Nilius, J.G. Hoenderop, and R.J. Bindels. 2004. Regulation of the mouse epithelial Ca2+ channel TRPV6 by the Ca2+-sensor calmodulin. J. Biol.

Chem. 279:28855-28861. 
Lee, J., and C.J. Rozell. 2017. Precision cell boundary tracking on DIC microscopy video for patch clamping. In 2017 IEEE International Conference on Acoustics, Speech and Signal Processing (ICASSP). IEEE. 1048-1052.

Levin, S.I., Z.M. Khaliq, T.K. Aman, T.M. Grieco, J.A. Kearney, I.M. Raman, and M.H. Meisler. 2006. Impaired motor function in mice with cell-specific knockout of sodium channel $\mathrm{Scn} 8 \mathrm{a}(\mathrm{Na}(\mathrm{V}) 1.6)$ in cerebellar Purkinje neurons and granule cells. J. Neurophysiol. 96:785-793.

Lewis, A.H., and I.M. Raman. 2011. Cross-species conservation of open-channel block by Na channel beta4 peptides reveals structural features required for resurgent Na current. $J$ Neurosci. 31:11527-11536.

Lewis, A.H., and I.M. Raman. 2013. Interactions among DIV voltage-sensor movement, fast inactivation, and resurgent $\mathrm{Na}$ current induced by the $\mathrm{NaV} \beta 4$ open-channel blocking peptide. J. Gen. Physiol. 142:191-206.

Li, Y.W., and D.A. Bayliss. 1998. Electrophysical properties, synaptic transmission and neuromodulation in serotonergic caudal raphé neurons. Clin. Exp. Pharmacol. Physiol. 25:468-473.

Li, M., J.W. West, Y. Lai, T. Scheuer, and W.A. Catterall. 1992. Functional modulation of brain sodium channels by cAMP-dependent phosphorylation. Neuron. 8:1151-1159.

Li, X., G. Wu, Y. Yang, S. Fu, X. Liu, H. Kang, X. Yang, X.-C. Su, and Y. Shen. 2017. Calmodulin dissociates the STIM1-Orai1 complex and STIM1 oligomers. Nat. Commun. 8:1042.

Lin, R.J., J. Bettencourt, J. White, D.J. Christini, and R.J. Butera. 2010. Real-time experiment interface for biological control applications. In Conf Proc IEEE Eng Med Biol Soc. IEEE. 4160-4163.

Lipkind, G.M., and H.A. Fozzard. 2000. KcsA crystal structure as framework for a molecular model of the Na+ channel pore. Biochem. 39:8161-8170.

Lipscombe, D., D.V. Madison, M. Poenie, H. Reuter, R.W. Tsien, and R.Y. Tsien. 1988. Imaging of cytosolic $\mathrm{Ca} 2+$ transients arising from $\mathrm{Ca} 2+$ stores and $\mathrm{Ca} 2+$ channels in sympathetic neurons. Neuron. 1:355-365.

Liu, C., S.D. Dib-Hajj, and S.G. Waxman. 2001. Fibroblast growth factor homologous factor 1B binds to the $\mathrm{C}$ terminus of the tetrodotoxin-resistant sodium channel rNav1.9a (NaN). $J$. Biol. Chem. 276:18925-18933. 
Liu, C.J., S.D. Dib-Hajj, M. Renganathan, T.R. Cummins, and S.G. Waxman. 2003. Modulation of the cardiac sodium channel Nav1.5 by fibroblast growth factor homologous factor 1B. J. Biol. Chem. 278:1029-1036.

Llinás, R., and M. Sugimori. 1980. Electrophysiological properties of in vitro Purkinje cell somata in mammalian cerebellar slices. J. Physiol. 305:171-195.

Long, S.B., E.B. Campbell, and R. MacKinnon. 2005. Crystal structure of a mammalian voltagedependent Shaker family K+ channel. Science. 309:897-903.

Lopez-Santiago, L.F., W.J. Brackenbury, C. Chen, and L.L. Isom. 2011. Na+ channel Scn1b gene regulates dorsal root ganglion nociceptor excitability in vivo. J. Biol. Chem. 286:2291322923.

Lorincz, A., and Z. Nusser. 2010. Molecular identity of dendritic voltage-gated sodium channels. Science. 328:906-909.

Lou, J.Y., F. Laezza, B.R. Gerber, M. Xiao, K.A. Yamada, H. Hartmann, A.M. Craig, J.M. Nerbonne, and D.M. Ornitz. 2005. Fibroblast growth factor 14 is an intracellular modulator of voltage - gated sodium channels. J. Physiol. 569:179-193.

MacDermott, A.B., M.L. Mayer, G.L. Westbrook, S.J. Smith, and J.L. Barker. 1986. NMDAreceptor activation increases cytoplasmic calcium concentration in cultured spinal cord neurones. Nature. 321:519.

Magee, J.C., and D. Johnston. 1997. A synaptically controlled, associative signal for Hebbian plasticity in hippocampal neurons. Science. 275:209-213.

Magistretti, J., L. Castelli, L. Forti, and E. D'Angelo. 2006. Kinetic and functional analysis of transient, persistent and resurgent sodium currents in rat cerebellar granule cells in situ: an electrophysiological and modelling study. J. Physiol. 573:83-106.

Maier, S.K., R.E. Westenbroek, K.A. Schenkman, E.O. Feigl, T. Scheuer, and W.A. Catterall. 2002. An unexpected role for brain-type sodium channels in coupling of cell surface depolarization to contraction in the heart. Proc. Natl. Acad. Sci. USA. 99:4073-4078.

Malenka, R.C. 1994. Synaptic plasticity in the hippocampus: LTP and LTD. Cell. 78:535-538.

Malinow, R., H. Schulman, and R.W. Tsien. 1989. Inhibition of postsynaptic PKC or CaMKII blocks induction but not expression of LTP. Science. 245:862-866.

Maravall, á., Z. Mainen, B. Sabatini, and K. Svoboda. 2000. Estimating intracellular calcium concentrations and buffering without wavelength ratioing. Biophys. J. 78:2655-2667.

Marder, E., and J.-M. Goaillard. 2006. Variability, compensation and homeostasis in neuron and network function. Nat. Rev. Neurosci. 7:563-574. 
Markram, H., J. Lübke, M. Frotscher, and B. Sakmann. 1997. Regulation of synaptic efficacy by coincidence of postsynaptic APs and EPSPs. Science. 275:213-215.

Marty, A., and E. Neher. 1995. Tight-seal whole-cell recording. In Single-channel recording. Springer. 31-52.

Maurice, N., T. Tkatch, M. Meisler, L.K. Sprunger, and D.J. Surmeier. 2001. D1/D5 dopamine receptor activation differentially modulates rapidly inactivating and persistent sodium currents in prefrontal cortex pyramidal neurons. J Neurosci. 21:2268-2277.

McAnelly, L., and H.H. Zakon. 1996. Protein kinase A activation increases sodium current magnitude in the electric organ of Sternopygus. J. Neurosci. 16:4383-4388.

Meech, R., and N. Standen. 1975. Potassium activation in Helix aspersa neurones under voltage clamp: a component mediated by calcium influx. J. Physiol. 249:211-259.

Menon, V., N. Spruston, and W.L. Kath. 2009. A state-mutating genetic algorithm to design ionchannel models. Proc. Natl. Acad. Sci. USA. 106:16829-16834.

Metzger, J.M., and R.L. Moss. 1990. Calcium-sensitive cross-bridge transitions in mammalian fast and slow skeletal muscle fibers. Science. 247:1088-1090.

Mickus, T., H. Jung, and N. Spruston. 1999. Properties of slow, cumulative sodium channel inactivation in rat hippocampal CA1 pyramidal neurons. Biophys. J. 76:846-860.

Middendorf, T.R., and R.W. Aldrich. 2017. Structural identifiability of equilibrium ligandbinding parameters. J. Gen. Physiol. 149:105-119.

Mihailescu, M., D. Krepkiy, M. Milescu, K. Gawrisch, K.J. Swartz, and S. White. 2014. Structural interactions of a voltage sensor toxin with lipid membranes. Proc. Natl. Acad. Sci. USA. 111:E5463-5470.

Milescu, L.S., G. Akk, and F. Sachs. 2005. Maximum likelihood estimation of ion channel kinetics from macroscopic currents. Biophys. J. 88:2494-2515.

Milescu, L.S., B.P. Bean, and J.C. Smith. 2010a. Isolation of somatic Na+ currents by selective inactivation of axonal channels with a voltage prepulse. J. Neurosci.

Milescu, L.S., K. Ptak, M. Mogri, T. Yamanishi, and J.C. Smith. 2008. Real-time kinetic modeling of voltage-gated ion channels using dynamic clamp. Biophys. J. 95:66-87.

Milescu, L.S., A. Yildiz, P.R. Selvin, and F. Sachs. 2006. Extracting dwell time sequences from processive molecular motor data. Biophys. J. 91:3135-3150.

Milescu, L.S., T. Yamanishi, K. Ptak, and J.C. Smith. 2010b. Kinetic properties and functional dynamics of sodium channels during repetitive spiking in a slow pacemaker neuron. $J$. Neurosci. 30:12113-12127. 
Milescu, L.S., C. Nicolai, and J. Bannen. 2000-2006. QuB. In. University at Buffalo, Buffalo, NY.

Milescu, M., J. Vobecky, S.H. Roh, S.H. Kim, H.J. Jung, J.I. Kim, and K.J. Swartz. 2007. Tarantula toxins interact with voltage sensors within lipid membranes. J. Gen. Physiol. 130:497-511.

Misonou, H., D.P. Mohapatra, E.W. Park, V. Leung, D. Zhen, K. Misonou, A.E. Anderson, and J.S. Trimmer. 2004. Regulation of ion channel localization and phosphorylation by neuronal activty. Nat. Neurosci. 7:711-718.

Misonou, H., and J.S. Trimmer. 2004. Determinants of voltage-gated potassium channel surface expression and localization in Mammalian neurons. Crit. Rev. Biochem Mol. Biol. 39:125-145.

Mitani, A., R. Sasaki, M. Oizumi, and T. Uka. 2013. A leaky-integrator model as a control mechanism underlying flexible decision making during task switching. PloS one. 8:e59670.

Miyawaki, A., J. Llopis, R. Heim, J.M. McCaffery, J.A. Adams, M. Ikura, and R.Y. Tsien. 1997. Fluorescent indicators for $\mathrm{Ca} 2+$ based on green fluorescent proteins and calmodulin. Nature. 388:882.

Moffatt, L. 2007. Estimation of ion channel kinetics from fluctuations of macroscopic currents. Biophys. J. 93:74-91.

Mohler, P.J., I. Rivolta, C. Napolitano, G. LeMaillet, S. Lambert, S.G. Priori, and V. Bennett. 2004. Nav1. 5 E1053K mutation causing Brugada syndrome blocks binding to ankyrin-G and expression of Nav1. 5 on the surface of cardiomyocytes. Proc. Natl. Acad. Sci. USA.. 101:17533-17538.

Nakahata, Y., and R. Yasuda. 2018. Plasticity of Spine Structure: Local Signaling, Translation and Cytoskeletal Reorganization. Front. Synaptic Neurosci. 10:29.

Nakai, J., M. Ohkura, and K. Imoto. 2001. A high signal-to-noise Ca 2+ probe composed of a single green fluorescent protein. Nat. Biotech. 19:137.

Navarro, M.A., J.V. Hibbard, M.E. Miller, T.W. Nivin, and L.S. Milescu. 2015. 3D Data Mapping and Real-Time Experiment Control and Visualization in Brain Slices. Biophys J. 109:1521-1527.

Navarro, M.A., A. Salari, M. Milescu, and L.S. Milescu. 2018. Estimating kinetic mechanisms with prior knowledge II: Behavioral constraints and numerical tests. J. Gen. Physiol. 150:339-354. 
Navarro, M.A., L.S. Milescu, and M. Milescu. 2019. Unlocking the gating mechanism of Kv2. 1 using guangxitoxin. J. Gen. Physiol. 151:275-278.

Nowotny, T., A. Szücs, R.D. Pinto, and A.I. Selverston. 2006. StdpC: a modern dynamic clamp. J. Neurosci. Meth. . 158:287-299.

O'Leary, T., A.H. Williams, J.S. Caplan, and E. Marder. 2013. Correlations in ion channel expression emerge from homeostatic tuning rules. Proc. Natl. Acad. Sci. USA. 110:E2645-E2654.

Ornitz, D.M. 2000. FGFs, heparan sulfate and FGFRs: complex interactions essential for development. Bioessays. 22:108-112.

Osorio, N., L. Cathala, M.H. Meisler, M. Crest, J. Magistretti, and P. Delmas. 2010. Persistent Nav1. 6 current at axon initial segments tunes spike timing of cerebellar granule cells. $J$. Physiol. 588:651-670.

Packer, A.M., L.E. Russell, H.W. Dalgleish, and M. Hausser. 2015. Simultaneous all-optical manipulation and recording of neural circuit activity with cellular resolution in vivo. Nat. Methods. 12:140-146.

Palay, S.L., C. Sotelo, A. Peters, and P.M. Orkand. 1968. The axon hillock and the initial segment. J. Cell Biol. 38:193-201.

Palmer, L.M., and G.J. Stuart. 2006. Site of action potential initiation in layer 5 pyramidal neurons. J. Neurosci. 26:1854-1863.

Pantazis, A., N. Savalli, D. Sigg, A. Neely, and R. Olcese. 2014. Functional heterogeneity of the four voltage sensors of a human L-type calcium channel. Proc. Natl. Acad. Sci. USA. 111:18381-18386.

Papadatos, G.A., P.M. Wallerstein, C.E. Head, R. Ratcliff, P.A. Brady, K. Benndorf, R.C. Saumarez, A.E. Trezise, C.L.-H. Huang, and J.I. Vandenberg. 2002. Slowed conduction and ventricular tachycardia after targeted disruption of the cardiac sodium channel gene Scn5a. Proc. Natl. Acad. Sci. USA. 99:6210-6215.

Pellerin, L., and P.J. Magistretti. 1994. Glutamate uptake into astrocytes stimulates aerobic glycolysis: a mechanism coupling neuronal activity to glucose utilization. Proc. Natl. Acad. Sci. USA. 91:10625-10629.

Penington, N.J., J.S. Kelly, and A.P. Fox. 1991. A study of the mechanism of Ca2+ current inhibition produced by serotonin in rat dorsal raphé neurons. J. Neurosci. 11:3594-3609.

Persechini, A., N.D. Moncrief, and R.H. Kretsinger. 1989. The EF-hand family of calciummodulated proteins. Trends Neurosci. 12:462-467. 
Peterson, B.Z., C.D. DeMaria, and D.T. Yue. 1999. Calmodulin is the Ca 2+ sensor for Ca 2+dependent inactivation of L-type calcium channels. Neuron. 22:549-558.

Pethig, R., M. Kuhn, R. Payne, E. Adler, T.-H. Chen, and L. Jaffe. 1989. On the dissociation constants of BAPTA-type calcium buffers. Cell calcium. 10:491-498.

Pologruto, T.A., B.L. Sabatini, and K. Svoboda. 2003. ScanImage: flexible software for operating laser scanning microscopes. Biomed. Eng. Online. 2:13.

Popescu, G., and A. Auerbach. 2003. Modal gating of NMDA receptors and the shape of their synaptic response. Nat. Neurosci. 6:476-483.

Portugues, R., M. Haesemeyer, M.L. Blum, and F. Engert. 2015. Whole-field visual motion drives swimming in larval zebrafish via a stochastic process. J. Exp. Biol. 218:1433-1443.

Press, W.H., S.A. Teukolsky, W.T. Vetterling, and B.P. Flannery. 1992. Numerical recipes in C. Cambridge University Press, Cambridge, MA.

Prinz, A.A., D. Bucher, and E. Marder. 2004. Similar network activity from disparate circuit parameters. Nat. Neurosci. 7:1345-1352.

Ptak, K., T. Yamanishi, J. Aungst, L.S. Milescu, R. Zhang, G.B. Richerson, and J.C. Smith. 2009. Raphé neurons stimulate respiratory circuit activity by multiple mechanisms via endogenously released serotonin and substance P. J. Neurosci. 29:3720-3737.

Qi, H., M.M. Moran, B. Navarro, J.A. Chong, G. Krapivinsky, L. Krapivinsky, Y. Kirichok, I.S. Ramsey, T.A. Quill, and D.E. Clapham. 2007. All four CatSper ion channel proteins are required for male fertility and sperm cell hyperactivated motility. Proc. Natl. Acad. Sci. USA. 104:1219-1223.

Qin, F., A. Auerbach, and F. Sachs. 1996. Estimating single channel kinetic parameters from idealized patch-clamp data containing missed events. Biophys. J. 70:264-280.

Qin, F., A. Auerbach, and F. Sachs. 2000a. A direct optimization approach to hidden markov modeling for single channel kinetics. Biophys. J. 79:1915-1927.

Qin, F., A. Auerbach, and F. Sachs. 2000b. Hidden Markov Modeling for Single Channel Kinetics with Filtering and Correlated Noise. Biophys. J. 79:1928-1944.

Qin, F., and L. Li. 2004. Model-based fitting of single-channel dwell-time distributions. Biophys. J. 87:1657-1671.

Qu, Y., R. Curtis, D. Lawson, K. Gilbride, P. Ge, P. DiStefano, I. Silos-Santiago, W. Catterall, and T. Scheuer. 2001. Differential modulation of sodium channel gating and persistent sodium currents by the $\beta 1, \beta 2$, and $\beta 3$ subunits. Mol. Cell. Neurosci. 18:570-580. 
Raman, I.M., and B.P. Bean. 1997. Resurgent sodium current and action potential formation in dissociated cerebellar Purkinje neurons. J. Neurosci. 17:4517-4526.

Raman, I.M., and B.P. Bean. 2001. Inactivation and recovery of sodium currents in cerebellar Purkinje neurons: evidence for two mechanisms. Biophys. J. 80:729-737.

Raman, I.M., L.K. Sprunger, M.H. Meisler, and B.P. Bean. 1997. Altered subthreshold sodium currents and disrupted firing patterns in Purkinje neurons of Scn8a mutant mice. Neuron. 19:881-891.

Ransdell, J.L., E. Dranoff, B. Lau, W.-L. Lo, D.L. Donermeyer, P.M. Allen, and J.M. Nerbonne. 2017. Loss of Navß4-mediated regulation of sodium currents in adult Purkinje neurons disrupts firing and impairs motor coordination and balance. Cell Rep. 19:532-544.

Rasband, M.N. 2010. The axon initial segment and the maintenance of neuronal polarity. Nat. Rev. Neurosci. 11:552-562.

Raue, A., C. Kreutz, T. Maiwald, J. Bachmann, M. Schilling, U. Klingmüller, and J. Timmer. 2009. Structural and practical identifiability analysis of partially observed dynamical models by exploiting the profile likelihood. Bioinformatics. 25:1923-1929.

Regehr, W.G., J.A. Connor, and D.W. Tank. 1989. Optical imaging of calcium accumulation in hippocampal pyramidal cells during synaptic activation. Nature. 341:533.

Remme, C.A., A.A. Wilde, and C.R. Bezzina. 2008. Cardiac sodium channel overlap syndromes: different faces of SCN5A mutations. Trends Cardiovasc. Med. 18:78-87.

Ren, D., B. Navarro, G. Perez, A.C. Jackson, S. Hsu, Q. Shi, J.L. Tilly, and D.E. Clapham. 2001a. A sperm ion channel required for sperm motility and male fertility. Nature. 413:603.

Ren, D., B. Navarro, H. Xu, L. Yue, Q. Shi, and D.E. Clapham. 2001b. A prokaryotic voltagegated sodium channel. Science. 294:2372-2375.

Richerson, G.B. 1997. Sudden infant death syndrome: the role of central chemosensitivity. Neuroscientist. 3:3-7.

Richerson, G.B. 2004. Serotonergic neurons as carbon dioxide sensors that maintain $\mathrm{pH}$ homeostasis. Nat. Rev. Neurosci. 5:449-461.

Richie, J. 1973. Energetic aspects of nerve conduction: the relationships between heat production, electrical activity and metabolism. Prog. Biophys. Mol. Biol. 26:147-187.

Robinson, H.P., and N. Kawai. 1993. Injection of digitally synthesized synaptic conductance transients to measure the integrative properties of neurons. J. Neurosci. Meth. 49:157165. 
Rosker, C., B. Lohberger, D. Hofer, B. Steinecker, S. Quasthoff, and W. Schreibmayer. 2007. The TTX Metabolite 4, 9-anhydro-TTX is a highly specific blocker of the Nav1. 6 voltage dependent sodium channel. Am. J. Physiol. Cell Physiol.

Rothberg, B.S., and K.L. Magleby. 2000. Voltage and Ca2+ activation of single largeconductance $\mathrm{Ca} 2+$-activated $\mathrm{K}+$ channels described by a two-tiered allosteric gating mechanism. J. Gen. Physiol. 116:75-99.

Rothberg, B.S., and K.L. Magleby. 2001. Testing for detailed balance (microscopic reversibility in ion channel gating. Biophys. J. 80:3025-3026.

Ruff, R.L., L. Simoncini, and W. Stüuhmer. 1988. Slow sodium channel inactivation in mammalian muscle: a possible role in regulating excitability. Muscle \& nerve. 11:502-510.

Rush, A.M., E.K. Wittmack, L. Tyrrell, J.A. Black, S.D. Dib - Hajj, and S.G. Waxman. 2006. Differential modulation of sodium channel Nav1. 6 by two members of the fibroblast growth factor homologous factor 2 subfamily. Eur. J. Neurosci. 23:2551-2562.

Sabatini, B.L., T.G. Oertner, and K. Svoboda. 2002. The life cycle of Ca 2+ ions in dendritic spines. Neuron. 33:439-452.

Salari, A., M.A. Navarro, and L.S. Milescu. 2016. Modeling the Kinetic Mechanisms of VoltageGated Ion Channels. In Advanced Patch-Clamp Analysis for Neuroscientists. A. Korngreen, editor. Humana Press. 267-304.

Salari, A., M.A. Navarro, M. Milescu, and L.S. Milescu. 2018. Estimating kinetic mechanisms with prior knowledge I: Linear parameter constraints. J. Gen. Physiol. 150:323-338.

Sarhan, M.F., C.-C. Tung, F. Van Petegem, and C.A. Ahern. 2012. Crystallographic basis for calcium regulation of sodium channels. Proc. Natl. Acad. Sci. USA. 109:3558-3563.

Schoppa, N.E., and F.J. Sigworth. 1998a. Activation of shaker potassium channels. I. Characterization of voltage-dependent transitions. J. Gen. Physiol. 111:271-294.

Schoppa, N.E., and F.J. Sigworth. 1998b. Activation of Shaker potassium channels. II. Kinetics of the V2 mutant channel. J. Gen. Physiol. 111:295-311.

Schoppa, N.E., and F.J. Sigworth. 1998c. Activation of Shaker potassium channels. III. An activation gating model for wild-type and V2 mutant channels. J. Gen. Physiol. 111:313342 .

Schulze, T.G., S.D. Detera-Wadleigh, N. Akula, A. Gupta, L. Kassem, J. Steele, J. Pearl, J. Strohmaier, R. Breuer, and M. Schwarz. 2009. Two variants in Ankyrin 3 (ANK3) are independent genetic risk factors for bipolar disorder. Molecular psychiatry. 14:487-491. 
Severson, C.A., W. Wang, V.A. Pieribone, C.I. Dohle, and G.B. Richerson. 2003. Midbrain serotonergic neurons are central pH chemoreceptors. Nat. Neurosci. 6:1139-1140.

Sharp, A.A., M.B. O'Neil, L.F. Abbott, and E. Marder. 1993. Dynamic clamp: computergenerated conductances in real neurons. J. Neurophysiol. 69:992-995.

Shu, Y., A. Duque, Y. Yu, B. Haider, and D.A. McCormick. 2007. Properties of action-potential initiation in neocortical pyramidal cells: evidence from whole cell axon recordings. $J$. Neurophysiol. 97:746-760.

Shull, G., C. Haffner, W.B. Huttner, E. Taverna, and S.B. Kodandaramaiah. 2018. Robotic platform for microinjection into single cells in intact tissue. bioRxiv.480004.

Siekmann, I., J. Sneyd, and E.J. Crampin. 2012. MCMC can detect nonidentifiable models. Biophys. J. 103:2275-2286.

Sigg, D. 2014. Modeling ion channels: Past, present, and future. J. Gen. Physiol. 144:7-26.

Sigworth, F. 1980. The variance of sodium current fluctuations at the node of Ranvier. J. Physiol. 307:97-129.

Sigworth, F.J. 1985. Open channel noise. I. Noise in acetylcholine receptor currents suggests conformational fluctuations. Biophys. J. 47:709-720.

Sigworth, F.J. 1994. Voltage gating of ion channels. Q. Rev. Biophys. . 27:1-40.

Sigworth, F.J. 1995. Design of the Epc-9, a Computer-Controlled Patch-Clamp Amplifier 1. Hardware. J. Neurosci. Meth. 56:195-202.

Sigworth, F.J. 2003. Structural biology: Life's transistors. Nature. 423:21.

Sigworth, F.J., and E. Neher. 1980. Single Na+ channel currents observed in cultured rat muscle cells. Nature. 287:447-449.

Smallwood, P.M., I. Munoz-Sanjuan, P. Tong, J.P. Macke, S.H. Hendry, D.J. Gilbert, N.G. Copeland, N.A. Jenkins, and J. Nathans. 1996. Fibroblast growth factor (FGF) homologous factors: new members of the FGF family implicated in nervous system development Proc. Natl. Acad. Sci. USA. 93:9850-9857.

Smith, J.C., H.H. Ellenberger, K. Ballanyi, D.W. Richter, and J.L. Feldman. 1991. Pre-Bötzinger complex: a brainstem region that may generate respiratory rhythm in mammals. Science. 254:726-729.

Smith, J.C., A.P. Abdala, H. Koizumi, I.A. Rybak, and J.F. Paton. 2007. Spatial and functional architecture of the mammalian brain stem respiratory network: a hierarchy of three oscillatory mechanisms. J. Neurophysiol. 98:3370-3387. 
Smith, S.B., L. Finzi, and C. Bustamante. 1992. Direct mechanical measurements of the elasticity of single DNA molecules by using magnetic beads. Science. 258:1122-1126.

Song, L., and K.L. Magleby. 1994. Testing for microscopic reversibility in the gating of maxi K+ channels using two-dimensional dwell-time distributions. Biophys. J. 67:91-104.

Squier, M.K., A.C. Miller, A.M. Malkinson, and J.J. Cohen. 1994. Calpain activation in apoptosis. J. Cell. Physiol. 159:229-237.

Stepanyuk, A., A. Borisyuk, and P. Belan. 2014. Maximum likelihood estimation of biophysical parameters of synaptic receptors from macroscopic currents. Front. Cell. Neurosci. 8.

Stepanyuk, A.R., A.L. Borisyuk, and P.V. Belan. 2011. Efficient maximum likelihood estimation of kinetic rate constants from macroscopic currents. PloS one. 6:e29731.

Strassberg, A.F., and L.J. DeFelice. 1993. Limitations of the Hodgkin-Huxley formalism: Effects of single channel kinetics on transmembrane voltage dynamics. Neural Computation. 5:843-855.

Stern, M. 1992. Buffering of calcium in the vicinity of a channel pore. Cell calcium. 13:183-192.

Strege, P.R., A.N. Holm, A. Rich, S.M. Miller, Y. Ou, M.G. Sarr, and G. Farrugia. 2003. Cytoskeletal modulation of sodium current in human jejunal circular smooth muscle cells. Am. J. Physiol. Cell Physiol. 284:C60-C66.

Strege, P.R., K. Knutson, S.J. Eggers, J.H. Li, F. Wang, D. Linden, J.H. Szurszewski, L. Milescu, A.B. Leiter, and G. Farrugia. 2017. Sodium channel Na V 1.3 is important for enterochromaffin cell excitability and serotonin release. Sci. Rep. 7:15650.

Strickland, D., and G. Mourou. 1985. Compression of amplified chirped optical pulses. Optics comm. 55:447-449.

Strickland, I.T., J.C. Martindale, P.L. Woodhams, A.J. Reeve, I.P. Chessell, and D.S. McQueen. 2008. Changes in the expression of NaV1.7, NaV1. 8 and NaV1. 9 in a distinct population of dorsal root ganglia innervating the rat knee joint in a model of chronic inflammatory joint pain. Eur. J. Pain. 12:564-572.

Stuart, G., J. Schiller, and B. Sakmann. 1997a. Action potential initiation and propagation in rat neocortical pyramidal neurons. J. Physiol. 505:617-632.

Stuart, G., N. Spruston, B. Sakmann, and M. Häusser. 1997b. Action potential initiation and backpropagation in neurons of the mammalian CNS. Trends Neurosci. 20:125-131.

Stühmer, W., F. Conti, H. Suzuki, X. Wang, M. Noda, N. Yahagi, H. Kubo, and S. Numa. 1989. Structural parts involved in activation and inactivation of the sodium channel. Nature. 339:597. 
Suk, H.-J., I. van Welie, S.B. Kodandaramaiah, B. Allen, C.R. Forest, and E.S. Boyden. 2017. Closed-loop real-time imaging enables fully automated cell-targeted patch-clamp neural recording in vivo. Neuron. 95:1037-1047. e1011.

Svoboda, K., C.F. Schmidt, B.J. Schnapp, and S.M. Block. 1993. Direct observation of kinesin stepping by optical trapping interferometry. Nature. 365:721-727.

Swartz, K.J. 2004. Towards a structural view of gating in potassium channels. Nat. Rev. Neurosci. 5:905-916.

Swartz, K.J., and R. MacKinnon. 1997a. Hanatoxin modifies the gating of a voltage-dependent $\mathrm{K}+$ channel through multiple binding sites. Neuron. 18:665-673.

Swartz, K.J., and R. MacKinnon. 1997b. Mapping the receptor site for hanatoxin, a gating modifier of voltage-dependent K+ channels. Neuron. 18:675-682.

Syed, S., F.E. Mullner, P.R. Selvin, and F.J. Sigworth. 2010. Improved Hidden Markov Models for Molecular Motors, Part 2 Extensions and Application to Experimental Data. Biophys. J. 99:3696-3703.

Taddese, A., and B.P. Bean. 2002. Subthreshold sodium current from rapidly inactivating sodium channels drives spontaneous firing of tuberomammillary neurons. Neuron. 33:587-600.

Tan, J., and D.M. Soderlund. 2011. Independent and joint modulation of rat Na v 1.6 voltagegated sodium channels by coexpression with the auxiliary $\beta 1$ and $\beta 2$ subunits. Biochem. Biophys. Res. Comm. 407:788-792.

Tan, R.C., and R.W. Joyner. 1990. Electrotonic influences on action potentials from isolated ventricular cells. Circul. Res. 67:1071-1081.

Tang, F., S. Lane, A. Korsak, J.F. Paton, A.V. Gourine, S. Kasparov, and A.G. Teschemacher. 2014. Lactate-mediated glia-neuronal signalling in the mammalian brain. Nat. Commun. $5: 3284$.

Tang, W., D.B. Halling, D. Black, P. Pate, J.-Z. Zhang, S. Pedersen, R.A. Altschuld, and S.L. Hamilton. 2003. Apocalmodulin and $\mathrm{Ca} 2+$ calmodulin-binding sites on the CaV1. 2 channel. Biophys. J. 85:1538-1547.

Teed, Z.R., and J.R. Silva. 2016. A computationally efficient algorithm for fitting ion channel parameters. MethodsX. 3:577-588.

Teleńczuk, M., B. Fontaine, and R. Brette. 2016. The basis of sharp spike onset in standard biophysical models. bioRxiv. 037549 . 
Tian, Y., P. Kongsuphol, M. Hug, J. Ousingsawat, R. Witzgall, R. Schreiber, and K.

Kunzelmann. 2011. Calmodulin-dependent activation of the epithelial calcium-dependent chloride channel TMEM16A. FASEB. 25:1058-1068.

Tilley, D.C., J.M. Angueyra, K.S. Eum, H. Kim, L.H. Chao, A.W. Peng, and J.T. Sack. 2018. The tarantula toxin GxTx detains $\mathrm{K}+$ channel gating charges in their resting conformation. $J$. Gen. Physiol.

Tuckwell, H.C., and N.J. Penington. 2014. Computational modeling of spike generation in serotonergic neurons of the dorsal raphé nucleus. Prog. Neurobiol. 118:59-101.

Vacher, H., D.P. Mohapatra, and J.S. Trimmer. 2008. Localization and targeting of voltagedependent ion channels in mammalian central neurons. Physiol. Rev. 88:1407-1447.

Vandenberg, C.A., and F. Bezanilla. 1991a. Single-channel, macroscopic, and gating currents from sodium channels in the squid giant axon. Biophys. J. 60:1499-1510.

Vandenberg, C.A., and F. Bezanilla. 1991b. A sodium channel gating model based on single channel, macroscopic ionic, and gating currents in the squid giant axon. Biophys. $J$. 60:1511-1533.

Vandermaelen, C., and G. Aghajanian. 1983. Electrophysiological and pharmacological characterization of serotonergic dorsal raphé neurons recorded extracellularly and intracellularly in rat brain slices. Brain Res. 289:109-119.

Venkataramanan, L., and F.J. Sigworth. 2002. Applying hidden Markov models to the analysis of single ion channel activity. Biophys. J. 82:1930-1942.

Venkatesan, K., Y. Liu, and M. Goldfarb. 2014. Fast-Onset Long-Term Open-State Block of Sodium Channels by A-type FHFs Mediates Classical Spike Accommodation in Hippocampal Pyramidal Neurons. J. Neurosci. 34:16126-16139.

Volk, D.W., J.N. Pierri, J.-M. Fritschy, S. Auh, A.R. Sampson, and D.A. Lewis. 2002. Reciprocal alterations in pre-and postsynaptic inhibitory markers at chandelier cell inputs to pyramidal neurons in schizophrenia. Cereb. Cortex. 12:1063-1070.

Wang, C., B.C. Chung, H. Yan, S.-Y. Lee, and G.S. Pitt. 2012. Crystal structure of the ternary complex of a NaV C-terminal domain, a fibroblast growth factor homologous factor, and calmodulin. Structure. 20:1167-1176.

Wang, C., C. Wang, E.G. Hoch, and G.S. Pitt. 2011. Identification of novel interaction sites that determine specificity between fibroblast growth factor homologous factors and voltagegated sodium channels. J. Biol. Chem. 286:24253-24263. 
Wang, G.K., and S.Y. Wang. 2003. Veratridine block of rat skeletal muscle Nav1. 4 sodium channels in the inner vestibule. J. Physiol. 548:667-675.

Wang, Q., D.G. McEwen, and D.M. Ornitz. 2000. Subcellular and developmental expression of alternatively spliced forms of fibroblast growth factor 14. Mech. Develop. 90:283-287.

Wang, W., S.R. Bradley, and G.B. Richerson. 2002. Quantification of the response of rat medullary raphé neurones to independent changes in pHo and PCO2. J. Physiol. 540:951-970.

Wang, W., J.H. Pizzonia, and G.B. Richerson. 1998. Chemosensitivity of rat medullary raphé neurones in primary tissue culture. J. Physiol. 511 ( Pt 2):433-450.

Wang, W., J.K. Tiwari, S.R. Bradley, R.V. Zaykin, and G.B. Richerson. 2001. Acidosisstimulated neurons of the medullary raphé are serotonergic. J. Neurophysiol. 85:22242235 .

Wen, H., and I.B. Levitan. 2002. Calmodulin is an auxiliary subunit of KCNQ2/3 potassium channels. J. Neurosci. 22:7991-8001.

West, J.W., R. Numann, B.J. Murphy, T. Scheuer, and W.A. Catterall. 1991. A phosphorylation site in the $\mathrm{Na}+$ channel required for modulation by protein kinase C. Science. 254:866868.

Wittmack, E.K., A.M. Rush, M.J. Craner, M. Goldfarb, S.G. Waxman, and S.D. Dib-Hajj. 2004. Fibroblast growth factor homologous factor 2B: Association with Na v1.6 and selective colocalization at nodes of Ranvier of dorsal root axons. J. Neurosci. 24:6765-6775.

Woodhull, A.M. 1973. Ionic blockage of sodium channels in nerve. J. Gen. Physiol. 61:687-708.

Wu, Q., I. Kolb, B.M. Callahan, Z. Su, W. Stoy, S.B. Kodandaramaiah, R. Neve, H. Zeng, E.S. Boyden, and C.R. Forest. 2016. Integration of autopatching with automated pipette and cell detection in vitro. J. Neurophysiol. 116:1564-1578.

Xia, J., and D. Ren. 2009. Egg coat proteins activate calcium entry into mouse sperm via CATSPER channels. Biol. Reproduc. 80:1092-1098.

Yamanishi, T., H. Koizumi, M.A. Navarro, L.S. Milescu, and J.C. Smith. 2018. Kinetic properties of persistent $\mathrm{Na}+$ current orchestrate oscillatory bursting in respiratory neurons. J. Gen. Physiol. 150:1523-1540.

Yamagata, T., I. Ogiwara, E. Mazaki, Y. Yanagawa, and K. Yamakawa. 2017. Nav1. 2 is expressed in caudal ganglionic eminence-derived disinhibitory interneurons: Mutually exclusive distributions of Nav1. 1 and Nav1. 2. Biochem. Biophys. Res. Commun. 491:1070-1076. 
Yamniuk, A.P., and H.J. Vogel. 2004. Calmodulin's flexibility allows for promiscuity in its interactions with target proteins and peptides. Mol. Biotech. 27:33-57.

Yan, H., J.L. Pablo, C. Wang, and G.S. Pitt. 2014. FGF14 modulates resurgent sodium current in mouse cerebellar Purkinje neurons. Elife. 3:e04193.

Yan, H., C. Wang, S.O. Marx, and G.S. Pitt. 2017. Calmodulin limits pathogenic Na+ channel persistent current. J. Gen. Physiol.jgp. 201611721.

Yang, Y., T. Adowski, B. Ramamurthy, A. Neef, and M.A. Xu-Friedman. 20415. High-speed dynamic-clamp interface. J. Neurophysiol. 113:2713-2720.

Yellen, G. 1998. The moving parts of voltage-gated ion channels. Q. Rev. Biophys. 31:239-295.

Yildiz, A., J.N. Forkey, S.A. McKinney, T. Ha, Y.E. Goldman, and P.R. Selvin. 2003. Myosin V walks hand-over-hand: single fluorophore imaging with 1.5-nm localization. Science. 300:2061-2065.

Yildiz, A., M. Tomishige, R.D. Vale, and P.R. Selvin. 2004. Kinesin walks hand-over-hand. Science. 303:676-678.

Yoshimura, T., and M.N. Rasband. 2014. Axon initial segments: diverse and dynamic neuronal compartments. Curr. Op. Neurobiol. 27:96-102.

Yu, Y., Y. Shu, and D.A. McCormick. 2008. Cortical action potential backpropagation explains spike threshold variability and rapid-onset kinetics. J. Neurosci. 28:7260-7272.

Yuste, R., and W. Denk. 1995. Dendritic spines as basic functional units of neuronal integration. Nature. 375:682.

Yuste, R., A. Majewska, and K. Holthoff. 2000. From form to function: calcium compartmentalization in dendritic spines. Nat. Neurosci. 3:653.

Zagotta, W.N., T. Hoshi, and R.W. Aldrich. 1994a. Shaker potassium channel gating. III: Evaluation of kinetic models for activation. J. Gen. Physiol. 103:321-362.

Zagotta, W.N., T. Hoshi, J. Dittman, and R.W. Aldrich. 1994b. Shaker potassium channel gating. II: Transitions in the activation pathway. J. Gen. Physiol. 103:279-319.

Zaydman, M.A., J.R. Silva, K. Delaloye, Y. Li, H. Liang, H.P. Larsson, J. Shi, and J. Cui. 2013. Kv7. 1 ion channels require a lipid to couple voltage sensing to pore opening. Proc. Natl. Acad. Sci. USA. 110:13180-13185. 
Marco Navarro was born in Chandler, AZ and grew up as the middle child, with an older sister Josefina and younger brother Aaron to loving parents Jesus and Angela. Marco has always enjoyed science and, as a member of his high school's Environmental Science club, brought recycling to the swimming pool complex. He lived in the Grand Canyon State for 19 years and never saw the Grand Canyon. For college, he moved to St. Louis, MO, to attend Saint Louis University, studying Biology, competing on the NCAA Div I Billiken Swim Team, and a member of the Micah service learning community. His first collegiate training trip was to the same pool he had trained during the previous seven years at Arizona State, but now was a guest for the first time. On that occasion, he took a trip with the SLU team to the Grand Canyon and found that it looked pretty much just like the many pictures he had seen. After experiencing a few major concussions, often occurring before organic chemistry exams, Marco had to retire from college swimming and used his time to learn Neuroscience. He joined a research lab in 2011 and began his own research project in his second year in the lab. His research mentor, Dr. Daniel Warren, convinced him to pursue a graduate education, to learn electrophysiology as a tool to understand physiology. During graduate school he has learned ion channel biophysics from great mentors, Lorin and Mirela, who have also broadened his worldview and helped him develop into a scientist. Marco's interest in understanding how nervous systems are maintained and organized to produce complex behaviors will likely never cease. For his next career stage, he will be joining the Sales and Support team at Scientifica LLC, a Neuroscience company, as an Advanced Systems Technical Support Engineer. 